\title{
Cooperative N-Heterocyclic Carbene/Palladium-catalyzed enantioselective umpolung annulations
}

\author{
Chang Guo, Mirco Fleige, Daniel Janssen-Müller, Constantin G. Daniliuc, Frank Glorius \\ Organisch-Chemisches Institut, Westfälische Wilhelms-Universität Münster, Corrensstraße 40, 48149 \\ Münster, Germany \\ glorius@uni-muenster.de
}

Table of Contents

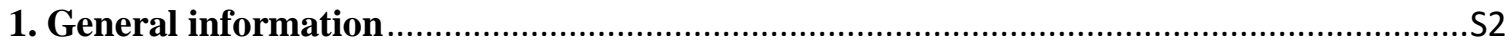

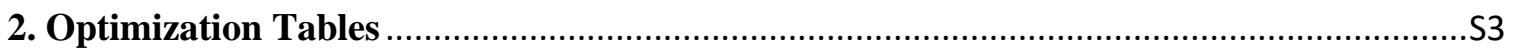

3. Synthesis and characterization of Vinyl benzoxazinanone ...............................................S4

4. Synthesis and Characterization of Products .......................................................................

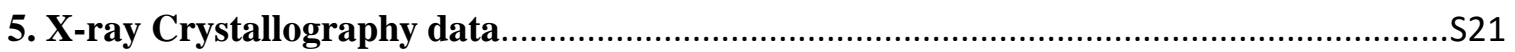

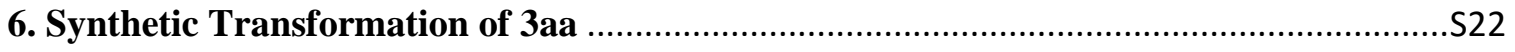

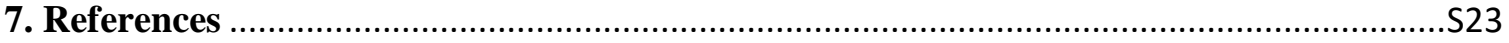

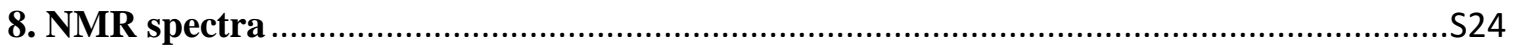

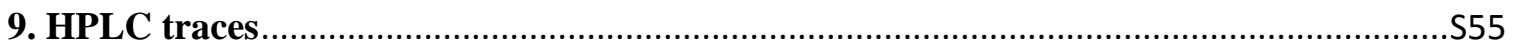




\section{General information}

Unless otherwise noted, all reactions were carried out under an atmosphere of argon in flamedried glassware. Reaction temperatures are reported as the temperature of the bath surrounding the vessel unless otherwise stated. The solvents used were purified by distillation over the drying agents indicated in parentheses and were transferred under argon: $n$-hexane $\left(\mathrm{CaH}_{2}\right)$, THF (Nabenzophenone), toluene $\left(\mathrm{CaH}_{2}\right)$. Analytical thin layer chromatography was performed on Polygram SIL G/UV254 plates. Flash chromatography was either performed on Merck silica gel (40-63 mesh) by standard technique eluting with solvents as indicated.

${ }^{1} \mathrm{H}$ and ${ }^{13} \mathrm{C}$-NMR spectra were recorded on a Bruker AV 300 or AV 400, Varian $500 \mathrm{MHz}$ INOVA or Varian Unity plus 600 in solvents as indicate. Chemical shifts $(\delta)$ are given in ppm relative to TMS. The residual solvent signals were used as references and the chemical shifts converted to the TMS scale $\left(\mathrm{CDCl}_{3}: \delta \mathrm{H}=7.26 \mathrm{ppm}, \delta \mathrm{C}=77.16 \mathrm{ppm}\right)$. ESI mass spectra were recorded on a Bruker Daltonics MicroTof. Specific rotation was measured on a Perkin Elmer 341 polarimeter at $20{ }^{\circ} \mathrm{C}$ using a quartz glass cell (100 mm path length). The Enantiomeric excesses (e.e.) was determined by HPLC analysis using chiral column OD, AS, IB, IC and AD. No attempts were made to optimize yields for substrate synthesis. Vinyl benzoxazinanone were synthesized according to the above procedures. ${ }^{1}$ 


\section{Optimization Tables}

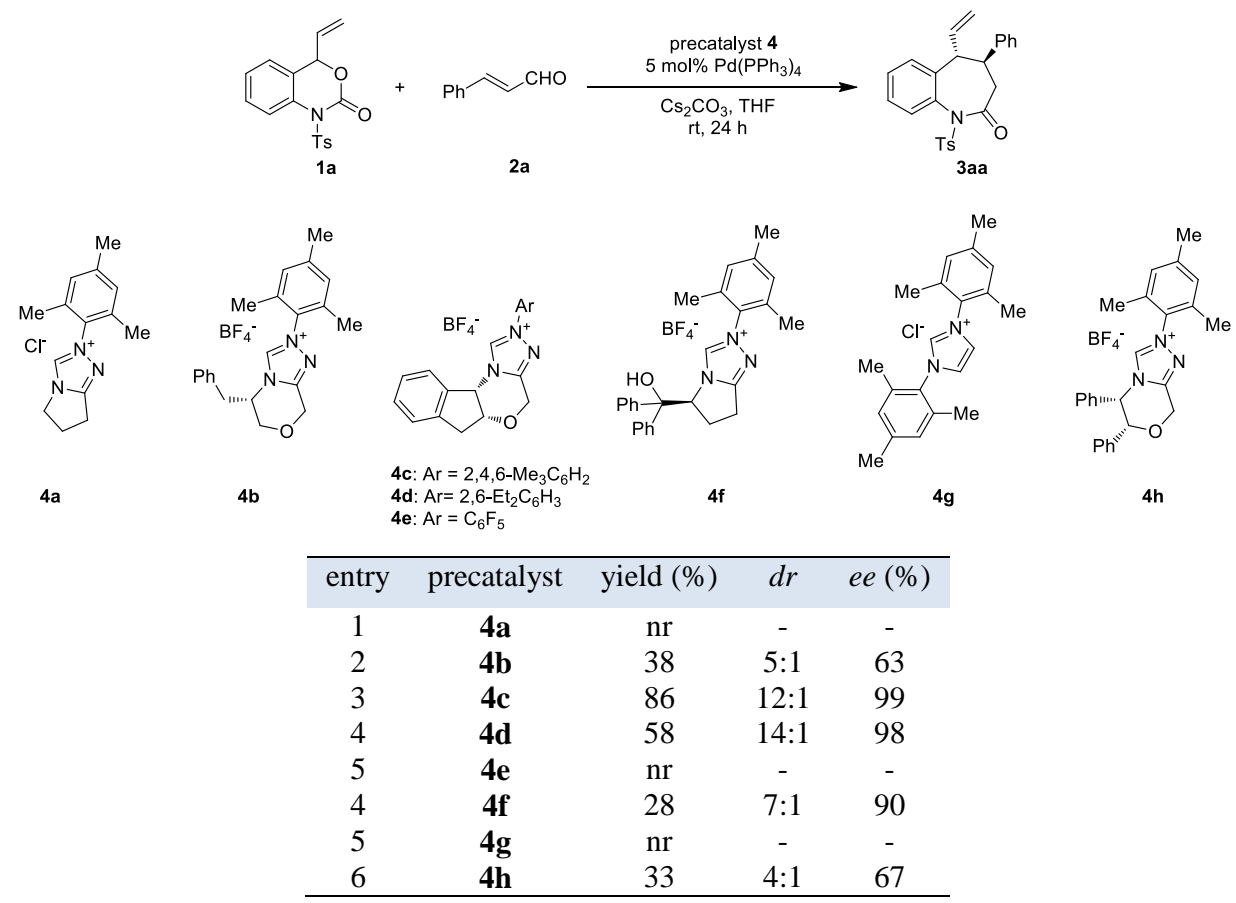

Figure S1. Optimization studies in the cooperative NHC-palladium catalyzed asymmetric umpolung annulation. Yields determined by ${ }^{1} \mathrm{H}$ NMR analysis using dibromomethane as internal standard. Reactions performed using 2.0 equiv. cinnamaldehyde on $0.1 \mathrm{mmol}$ scale. The ee value of 3aa was determined by HPLC using a chiral column.

As shown in Figure S1, NHC-4c is the best organocatalyst for the cooperative reaction.

\begin{tabular}{|c|c|c|c|c|c|}
\hline & & $\approx \mathrm{CHO}$ & $\begin{array}{l}\% \text { precatalyst 4c } \\
\left.1 \% \mathrm{Pd}^{2} \mathrm{PPh}_{3}\right)_{4} \\
\text { se, solvent } \\
\text { rt, } 24 \mathrm{~h}\end{array}$ & & 3аa \\
\hline entry & base & solvent & Yield (\%) & $d r$ & $e e(\%)$ \\
\hline 1 & $\mathrm{Cs}_{2} \mathrm{CO}_{3}$ & THF & 86 & $12: 1$ & 99 \\
\hline 2 & DBU & THF & 63 & $12: 1$ & 97 \\
\hline 3 & $\mathrm{~K}_{2} \mathrm{CO}_{3}$ & THF & 51 & $11: 1$ & 96 \\
\hline 4 & $\mathrm{Cs}_{2} \mathrm{CO}_{3}$ & toluene & 56 & $12: 1$ & 96 \\
\hline 5 & $\mathrm{Cs}_{2} \mathrm{CO}_{3}$ & $\mathrm{CHCl}_{3}$ & 29 & $15: 1$ & 94 \\
\hline 6 & $\mathrm{Cs}_{2} \mathrm{CO}_{3}$ & $\mathrm{Et}_{2} \mathrm{O}$ & trace & - & - \\
\hline 7 & $\mathrm{Cs}_{2} \mathrm{CO}_{3}$ & 1,4-dioxane & 59 & $10: 1$ & 97 \\
\hline 8 & $\mathrm{Cs}_{2} \mathrm{CO}_{3}$ & DME & 85 & $12: 1$ & 98 \\
\hline
\end{tabular}

Figure S2. Evaluation of base and solvent in the NHC-palladium catalyzed asymmetric umpolung annulation. Yields determined by ${ }^{1} \mathrm{H}$ NMR analysis using dibromomethane as internal standard. Reactions performed using 2.0 equiv. cinnamaldehyde on $0.1 \mathrm{mmol}$ scale. The ee value of 3aa was determined by HPLC using a chiral column.

As shown in Figure $\mathbf{S 2}, \mathrm{Cs}_{2} \mathrm{CO}_{3}$ and THF were best condition for the cooperative reaction. 


\section{Synthesis and characterization of Vinyl benzoxazinanone ${ }^{1}$}

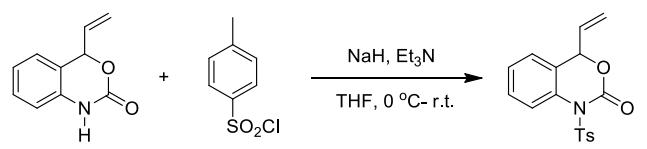

4-vinyl-1H-benzo[d][1,3]oxazin-2(4H)-one $(20 \mathrm{mmol})$ and $\mathrm{Et}_{3} \mathrm{~N}$ (30 mmol) were stirred in THF $(150 \mathrm{~mL})$ at $0{ }^{\circ} \mathrm{C}$. Then $\mathrm{NaH}(22 \mathrm{mmol})$ was added slowly to the mixture and stirred at r.t. for $1 \mathrm{~h}$. The reaction mixture was added 4-toluenesulfonyl chloride $(30 \mathrm{mmol})$. After $4 \mathrm{~h}$, the reaction was diluted with $100 \mathrm{~mL}$ of $\mathrm{H}_{2} \mathrm{O}$ and extracted three times with dichloromethane. The combined organic phases were dried over $\mathrm{Na}_{2} \mathrm{SO}_{4}$, filtered, and concentrated under reduced pressure. The crude solid was purified by chromatography on silica gel to affording $\mathbf{1 a}$.

Other benzoxazinanone were synthesized according to the above procedures.

\section{7-methyl-1-tosyl-4-vinyl-1H-benzo[d][1,3]oxazin-2(4H)-one (2b)}

${ }^{1}$ H NMR (300 MHz, CDCl $): \delta 8.09-8.06(\mathrm{~m}, 2 \mathrm{H}), 7.44-7.37(\mathrm{~m}, 3 \mathrm{H}), 7.07-$ $7.06(\mathrm{~m}, 2 \mathrm{H}), 6.08-5.96(\mathrm{~m}, 1 \mathrm{H}), 5.60(\mathrm{~d}, J=6.0 \mathrm{~Hz}, 1 \mathrm{H}), 5.46-5.31(\mathrm{~m}, 2 \mathrm{H})$, $2.47(\mathrm{~s}, 3 \mathrm{H}), 2.42(\mathrm{~s}, 3 \mathrm{H}) .{ }^{13} \mathbf{C}$ NMR $\left(75 \mathrm{MHz}, \mathrm{CDCl}_{3}\right): \delta 149.3,145.5,139.5,135.7$, 133.9, 132.3, 129.6, 129.1, 126.7, 124.9, 123.5, 121.4, 121.0, 79.5, 21.8, 21.7. ATR-FTIR (cm $\left.{ }^{-1}\right)$ : 3649, 1740, 1717, 1616, 1593, 1508, 1458, 1335, 1184, 1161, 1080, 988, 938, 864, 748, 682. ESI-MS: calculated: $\left[\mathrm{C}_{18} \mathrm{H}_{17} \mathrm{NO}_{4} \mathrm{~S}+\mathrm{Na}\right]^{+}: 366.0770$, found: 366.0784 .

\section{7-fluoro-1-tosyl-4-vinyl-1H-benzo[d][1,3]oxazin-2(4H)-one (2d)}

${ }^{1}$ H NMR (300 MHz, CDCl $): \delta 8.13$ - 8.05 (m, 2H), $7.43-7.35$ (m, 3H), 7.16 (dd,

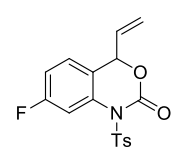
$J=8.5,5.7 \mathrm{~Hz}, 1 \mathrm{H}), 6.97(\mathrm{td}, J=8.3,2.4 \mathrm{~Hz}, 1 \mathrm{H}), 6.11-5.93(\mathrm{~m}, 1 \mathrm{H}), 5.63(\mathrm{~d}, J=$ $6.1 \mathrm{~Hz}, 1 \mathrm{H}), 5.53-5.31(\mathrm{~m}, 2 \mathrm{H}), 2.47(\mathrm{~s}, 3 \mathrm{H}) .{ }^{13} \mathrm{C} \mathrm{NMR}\left(75 \mathrm{MHz}, \mathrm{CDCl}_{3}\right): \delta$ $162.69(\mathrm{~d}, J=248.0 \mathrm{~Hz}), 148.87,146.06,135.46$ (d, $J=11.2 \mathrm{~Hz}), 135.40,131.96,129.94,129.24$, $126.53(\mathrm{~d}, J=9.6 \mathrm{~Hz}), 122.31(\mathrm{~d}, J=3.1 \mathrm{~Hz}), 121.67,113.05(\mathrm{~d}, J=22.1 \mathrm{~Hz}), 109.05$ (d, $J=$ $27.7 \mathrm{~Hz}), 79.19,21.91 ;{ }^{19} \mathbf{F}$ NMR (282 $\left.\mathbf{M H z}, \mathbf{C D C l}_{3}\right): \delta$ - 109.8; ATR-FTIR (cm-1): 3649, 1759 , 1597, 1435, 1373, 1165, 1049, 988, 949, 856, 837, 752, 671. ESI-MS: calculated: $\left[\mathrm{C}_{17} \mathrm{H}_{14} \mathrm{FNO}_{4} \mathrm{~S}\right.$ $+\mathrm{Na}]^{+}: 370.0520$, found: 370.0514 . 
1-tosyl-7-(trifluoromethyl)-4-vinyl-1H-benzo[d][1,3]oxazin-2(4H)-one (2f)

${ }^{1}$ H NMR (600 MHz, $\left.\mathbf{C D C l}_{3}\right): \delta 8.10-8.08(\mathrm{~m}, 2 \mathrm{H}), 7.91$ (br s, 1H), $7.55-7.50$ (m, 1H), $7.42-7.39(\mathrm{~m}, 2 \mathrm{H}), 7.34(\mathrm{~d}, J=7.9 \mathrm{~Hz}, 1 \mathrm{H}), 6.05-5.99(\mathrm{~m}, 1 \mathrm{H}), 5.70-$ $5.67(\mathrm{~m}, 1 \mathrm{H}), 5.50(\mathrm{dd}, J=10.5,1.2 \mathrm{~Hz}, 1 \mathrm{H}), 5.38(\mathrm{dd}, J=17.2,1.4 \mathrm{~Hz}, 1 \mathrm{H}), 2.48$ $(\mathrm{s}, 3 \mathrm{H}) ;{ }^{13} \mathbf{C}$ NMR $\left(151 \mathrm{MHz}, \mathrm{CDCl}_{3}\right): \delta 148.59,146.27,135.22,134.69,131.86$ (q, $\left.J=33.2 \mathrm{~Hz}\right)$, $131.45,130.11(\mathrm{q}, J=1.2 \mathrm{~Hz}), 129.98,129.78,125.94,123.44(\mathrm{q}, J=272.7 \mathrm{~Hz}), 122.94(\mathrm{q}, J=$ $3.7 \mathrm{~Hz}), 122.02,118.15$ (q, $J=4.0 \mathrm{~Hz}), 79.07,21.89 ;{ }^{19} \mathbf{F}$ NMR (564 MHz, $\left.\mathbf{C D C l}_{3}\right) \delta$ - 62.7. ATR-FTIR (cm-1): 3649, 1759, 1597, 1366, 1327, 1126, 1049, 988, 945, 856, 833, 814, 752, 729, 656. ESI-MS: calculated: $\left[\mathrm{C}_{18} \mathrm{H}_{14} \mathrm{~F}_{3} \mathrm{NO}_{4} \mathrm{~S}+\mathrm{Na}\right]^{+}$: 420.0488, found: 420.0482 .

\section{6-methyl-1-tosyl-4-vinyl-1H-benzo[d][1,3]oxazin-2(4H)-one (2g)}

${ }^{1}$ H NMR (300 MHz, CDCl $): \delta 8.09(\mathrm{~d}, J=8.4 \mathrm{~Hz}, 2 \mathrm{H}), 7.50(\mathrm{~d}, J=8.4 \mathrm{~Hz}, 1 \mathrm{H})$, $7.39(\mathrm{~d}, J=8.1 \mathrm{~Hz}, 2 \mathrm{H}), 7.25-7.19(\mathrm{~m}, 1 \mathrm{H}), 6.98(\mathrm{~s}, 1 \mathrm{H}), 6.08-5.97(\mathrm{~m}, 1 \mathrm{H}), 5.60$ $(\mathrm{d}, J=6.1 \mathrm{~Hz}, 1 \mathrm{H}), 5.47-5.32(\mathrm{~m}, 2 \mathrm{H}), 2.46(\mathrm{~s}, 3 \mathrm{H}), 2.36(\mathrm{~s}, 3 \mathrm{H}) ;{ }^{13} \mathbf{C} \mathbf{N M R}$ (75 MHz, $\mathbf{C D C l}_{3}$ ): $\delta$ 149.5, 145.6, 136.1, 135.9, 132.3, 131.7, 129.9, 129.8, 129.2, 126.5, 125.7, 121.3, 120.7, 79.7, 21.9, 21.0; ATR-FTIR (cm $\left.{ }^{-1}\right): 3649,1748,1508,1497,1362,1339,1234$, 1184, 1165, 1084, 1042, 945, 876, 706, 667. ESI-MS: calculated: $\left[\mathrm{C}_{18} \mathrm{H}_{17} \mathrm{NO}_{4} \mathrm{~S}+\mathrm{Na}\right]^{+}: 366.0770$, found: 366.0778 .

\section{6-chloro-1-tosyl-4-vinyl-1H-benzo[d][1,3]oxazin-2(4H)-one (2h)}

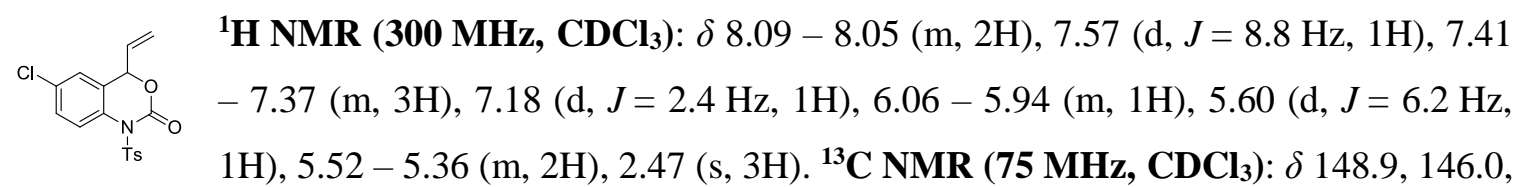
$135.4,132.7,131.8,131.5,129.9,129.4,129.3,128.3,125.3,122.3,122.1,79.0,21.9$. ATRFTIR $\left(\mathbf{c m}^{-1}\right): 3649,1748,1717,1637,1559,1420,1362,1346,1296,1165,1084,1030,930,887$, 860, 814, 733, 687, 664. ESI-MS: calculated: $\left[\mathrm{C}_{17} \mathrm{H}_{14} \mathrm{ClNO}_{4} \mathrm{~S}+\mathrm{Na}\right]^{+}: 386.0224$,found: 386.0221 .

\section{6-fluoro-1-tosyl-4-vinyl-1H-benzo[d][1,3]oxazin-2(4H)-one (2j)}

${ }^{1}$ H NMR (300 MHz, CDCl $): \delta 8.10-8.05$ (m, 2H), $7.59(\mathrm{dd}, J=9.1,4.5 \mathrm{~Hz}, 1 \mathrm{H})$,

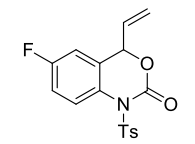
$7.39(\mathrm{~d}, J=8.2 \mathrm{~Hz}, 2 \mathrm{H}), 7.12(\mathrm{td}, J=8.6,2.9 \mathrm{~Hz}, 1 \mathrm{H}), 6.92(\mathrm{dd}, J=7.8,2.9 \mathrm{~Hz}$, $1 \mathrm{H}), 6.06-5.94(\mathrm{~m}, 1 \mathrm{H}), 5.60(\mathrm{~d}, J=6.2 \mathrm{~Hz}, 1 \mathrm{H}), 5.52-5.36(\mathrm{~m}, 2 \mathrm{H}), 2.47(\mathrm{~s}, 3 \mathrm{H})$;

${ }^{13} \mathrm{C}$ NMR $\left(75 \mathrm{MHz}, \mathrm{CDCl}_{3}\right): \delta 160.25(\mathrm{~d}, J=248.0 \mathrm{~Hz}), 149.02,145.95,135.52,131.47,130.21$ $(\mathrm{d}, J=3.3 \mathrm{~Hz}), 129.89,129.19,128.82(\mathrm{~d}, J=7.7 \mathrm{~Hz}), 122.83(\mathrm{~d}, J=8.4 \mathrm{~Hz}), 122.02,116.26(\mathrm{~d}$, 
$J=23.2 \mathrm{~Hz}), 112.41(\mathrm{~d}, J=24.5 \mathrm{~Hz}), 79.06(\mathrm{~d}, J=1.9 \mathrm{~Hz}), 21.90 .{ }^{19} \mathbf{F}$ NMR (282 $\left.\mathbf{M H z}, \mathbf{C D C l}_{\mathbf{3}}\right)$ : $\delta$ - 114.9. ATR-FTIR $\left(\mathbf{c m}^{-1}\right): 3649,1740,1543,1508,1350,1296,1261,1231,1196,1084$, 1049, 976, 953, 937, 899, 818, 764, 656. ESI-MS: calculated: $\left[\mathrm{C}_{17} \mathrm{H}_{14} \mathrm{FNO}_{4} \mathrm{~S}+\mathrm{Na}\right]^{+}:$370.0520, found: 370.0523 .

6-methoxy-1-tosyl-4-vinyl-1H-benzo[d][1,3]oxazin-2(4H)-one (2k)

${ }^{1}$ H NMR (300 MHz, $\left.\mathbf{C D C l}_{3}\right): \delta 8.09-8.05(\mathrm{~m}, 2 \mathrm{H}), 7.52(\mathrm{~d}, J=9.0 \mathrm{~Hz}, 1 \mathrm{H})$, $\begin{array}{ll}\underbrace{}_{\mathrm{N}} \AA_{\mathrm{s}} & 7.39-7.26(\mathrm{~m}, 2 \mathrm{H}), 6.93(\mathrm{dd}, J=9.0,2.9 \mathrm{~Hz}, 1 \mathrm{H}), 6.70(\mathrm{~d}, J=2.8 \mathrm{~Hz}, 1 \mathrm{H}), 6.06 \\ & -5.95(\mathrm{~m}, 1 \mathrm{H}), 5.58(\mathrm{~d}, J=6.3 \mathrm{~Hz}, 1 \mathrm{H}), 5.47-5.34(\mathrm{~m}, 2 \mathrm{H}), 3.81(\mathrm{~s}, 3 \mathrm{H}), 2.46(\mathrm{~s},\end{array}$ 3H); ${ }^{13} \mathbf{C}$ NMR (75 MHz, $\left.\mathbf{C D C l}_{3}\right): \delta 157.6,149.4,145.6,135.9,132.1,129.8,129.1,128.1,127.1$, 122.3, 121.5, 114.3, 110.7, 79.7, 55.8, 21.9; ATR-FTIR (cm $\left.{ }^{-1}\right): 3649,1732,1717,1686,1489$, $1458,1435,1420,1397,1234,1088,945,887,853,826,764,741$, 702. ESI-MS: calculated: $\left[\mathrm{C}_{18} \mathrm{H}_{17} \mathrm{NO}_{5} \mathrm{SNa}\right]^{+}=382.0720$; found: 382.0718 . 


\section{Synthesis and Characterization of Products}

A dried and argon-filled Schlenk flask was charged with vinyl benzoxazinanone 1a $(0.2 \mathrm{mmol}$, 1.0 equiv) and $\mathrm{Cs}_{2} \mathrm{CO}_{3}\left(0.2 \mathrm{mmol}, 1.0\right.$ equiv), $\mathrm{Pd}\left(\mathrm{PPh}_{3}\right)_{4}(0.01 \mathrm{mmol}, 5 \mathrm{~mol} \%)$ and triazolium salt 4c $(0.03 \mathrm{mmol}, 15 \mathrm{~mol} \%)$. Then, enal 2a $(0.4 \mathrm{mmol})$ was added quickly to the mixture. Subsequently, $3 \mathrm{~mL}$ THF was added to the mixture. The mixture was stirred at r.t. for $24 \mathrm{~h}$. After purification by column chromatography on silica gel (Pentane: Ethyl acetate $=6: 1$ ) the desired product 3aa was obtained.

\section{Analytical Data for the Products}

(4S,5R)-4-phenyl-1-tosyl-5-vinyl-4,5-dihydro-1H-benzo[b]azepin-2(3H)-one (3aa)

Total yield: $71 \mathrm{mg}(86 \%) ;{ }^{1} \mathbf{H}$ NMR (400 MHz, $\left.\mathbf{C D C l}_{3}\right) \delta 8.02(\mathrm{~d}, J=8.3 \mathrm{~Hz}, 2 \mathrm{H})$, $7.53-7.46(\mathrm{~m}, 1 \mathrm{H}), 7.41-7.28(\mathrm{~m}, 5 \mathrm{H}), 7.11-6.97(\mathrm{~m}, 3 \mathrm{H}), 6.56-6.46(\mathrm{~m}, 2 \mathrm{H})$,

$5.81(\mathrm{ddd}, J=17.3,10.4,8.1 \mathrm{~Hz}, 1 \mathrm{H}), 5.00(\mathrm{~d}, J=10.7 \mathrm{~Hz}, 1 \mathrm{H}), 4.74(\mathrm{~d}, J=17.2 \mathrm{~Hz}$, 1H), 3.57 (dd, $J=12.0,8.2 \mathrm{~Hz}, 1 \mathrm{H}), 2.98$ (dd, $J=11.9,8.0 \mathrm{~Hz}, 1 \mathrm{H}), 2.53-2.39$ (m, 4H), $2.14-$ $2.06(\mathrm{~m}, 1 \mathrm{H}) .{ }^{13} \mathbf{C}$ NMR (101 $\left.\mathbf{M H z}, \mathbf{C D C l}_{3}\right) \delta 169.98,145.30,141.73,138.74,136.19,135.77$, $135.59,129.90,129.28,129.24,129.15,128.39$, 127.40, 127.32, 126.96, 126.63, 119.13, 50.65,

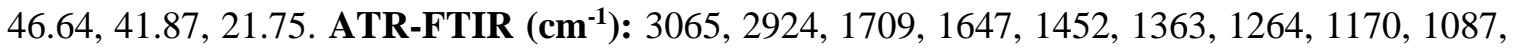
733, 699, 659; ESI-MS: calculated $\left[\mathrm{C}_{25} \mathrm{H}_{23} \mathrm{NO}_{3} \mathrm{~S}+\mathrm{Na}^{+}:\right.$440.1291, found: 440.1290; $[\alpha]^{26} \mathbf{D}=+11.2\left(\mathrm{c}=1.2, \quad \mathrm{CHCl}_{3}\right)$; The product was analyzed by HPLC to determine the enantiomeric excess: $99 \%$ ee $(\mathrm{AD}-\mathrm{H}$, hexane/i-PrOH $=70 / 30$, detector: $230 \mathrm{~nm}$, flow rate: 1 $\mathrm{mL} / \mathrm{min}), \mathrm{t}_{1}($ minor $)=12.4 \mathrm{~min}, \mathrm{t}_{2}$ (major) $=13.8 \mathrm{~min}$.

(4S,5R)-4-(p-tolyl)-1-tosyl-5-vinyl-4,5-dihydro-1H-benzo[b]azepin-2(3H)-one (3ab)

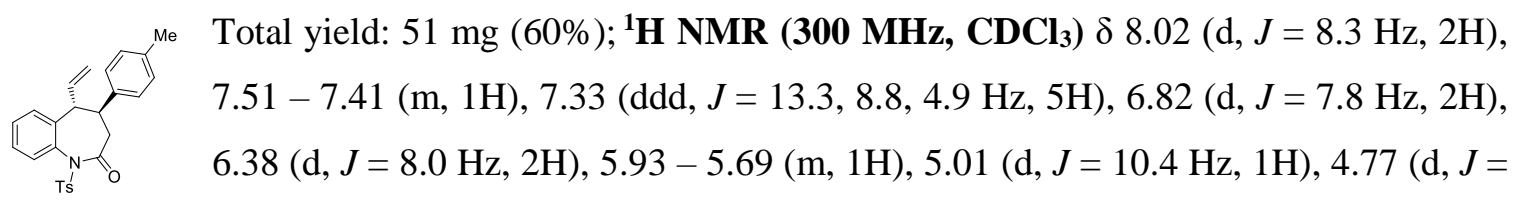
$17.2 \mathrm{~Hz}, 1 \mathrm{H}), 3.56(\mathrm{dd}, J=11.9,8.1 \mathrm{~Hz}, 1 \mathrm{H}), 2.96(\mathrm{dd}, J=11.9,8.2 \mathrm{~Hz}, 1 \mathrm{H}), 2.51-2.38(\mathrm{~m}, 4 \mathrm{H})$, 2.19 (s, 3H), $2.06(\mathrm{~d}, J=12.8 \mathrm{~Hz}, 1 \mathrm{H}) .{ }^{13} \mathbf{C} \mathbf{~ N M R}\left(\mathbf{7 5} \mathbf{M H z}, \mathbf{C D C l}_{3}\right) \delta$ 170.03, 145.29, 138.85, $138.67,136.49,136.20,135.79,135.74,129.90,129.29,129.23,129.13,127.37,127.12$, 126.68, 119.13, 50.22, 46.44, 42.14, 21.78, 21.05. ATR-FTIR (cm-1): 2924, 2854, 1713, 1455, 1365, 1172, 1123, 1088, 924, 815, 762, 663; ESI-MS: calculated $\left[\mathrm{C}_{26} \mathrm{H}_{25} \mathrm{NO}_{3} \mathrm{~S}+\mathrm{Na}\right]^{+}$: 454.1447, found: $454.1457 ;[\alpha]^{26} \mathbf{D}=+23.5\left(\mathrm{c}=1.2, \mathrm{CHCl}_{3}\right)$; The product was analyzed by HPLC to determine the 
enantiomeric excess: $97 \%$ ee $(\mathrm{AD}-\mathrm{H}$, hexane $/ i-\mathrm{PrOH}=70 / 30$, detector: $230 \mathrm{~nm}$, flow rate: 1 $\mathrm{mL} / \mathrm{min}), \mathrm{t}_{1}($ minor $)=10.5 \min , \mathrm{t}_{2}($ major $)=11.9 \min$.

(4S,5R)-4-(4-methoxyphenyl)-1-tosyl-5-vinyl-4,5-dihydro-1H-benzo[b]azepin-2(3H)-one (3ac)

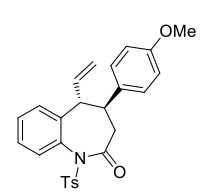

Total yield: $73 \mathrm{mg}$ (82\%); ${ }^{1} \mathbf{H}$ NMR (300 $\left.\mathbf{M H z}, \mathbf{C D C l}_{3}\right) \delta 8.02(\mathrm{~d}, J=8.4 \mathrm{~Hz}, 2 \mathrm{H})$, $7.52-7.44(\mathrm{~m}, 1 \mathrm{H}), 7.40-7.24(\mathrm{~m}, 5 \mathrm{H}), 6.59-6.51(\mathrm{~m}, 2 \mathrm{H}), 6.42(\mathrm{~d}, J=8.7 \mathrm{~Hz}$, 2H), $5.90-5.73(\mathrm{~m}, 1 \mathrm{H}), 5.01(\mathrm{~d}, J=10.3 \mathrm{~Hz}, 1 \mathrm{H}), 4.75(\mathrm{~d}, J=17.2 \mathrm{~Hz}, 1 \mathrm{H}), 3.67$ (s, 3H), $3.51(\mathrm{dt}, J=15.0,7.5 \mathrm{~Hz}, 1 \mathrm{H}), 2.95(\mathrm{dd}, J=11.9,7.9 \mathrm{~Hz}, 1 \mathrm{H}), 2.53-2.39(\mathrm{~m}, 4 \mathrm{H}), 2.06$ $(\mathrm{dd}, J=12.8,0.9 \mathrm{~Hz}, 1 \mathrm{H}) .{ }^{13} \mathbf{C}$ NMR $\left(\mathbf{7 5} \mathbf{~ M H z}, \mathbf{C D C l}_{3}\right) \delta 170.14,158.32,145.29,138.87,136.22$, $135.75,133.89,129.90,129.29,129.23,129.12$, 128.28, 127.37, 126.65, 119.11, 113.74, 55.10, 49.88, 46.84, 42.17, 21.77. ATR-FTIR (cm $\left.{ }^{-1}\right):$ 2945, 1699, 1650, 1517, 1362, 1261, 998, 915 , 752, 732, 670; ESI-MS: calculated $\left[\mathrm{C}_{26} \mathrm{H}_{25} \mathrm{NO}_{4} \mathrm{~S}+\mathrm{Na}\right]^{+}:$470.1397, found: 470.1403; $[\alpha]^{26}{ }_{D}=+22.6\left(\mathrm{c}=1.7, \mathrm{CHCl}_{3}\right)$; The product was analyzed by HPLC to determine the enantiomeric excess: $98 \%$ ee $(\mathrm{AD}-\mathrm{H}$, hexane $/ i-\mathrm{PrOH}=70 / 30$, detector: $230 \mathrm{~nm}$, flow rate: 1 $\mathrm{mL} / \mathrm{min}), \mathrm{t}_{1}($ minor $)=14.3$ min, $\mathrm{t}_{2}($ major $)=21.6 \min$.

(4S,5R)-4-(4-(dimethylamino)phenyl)-1-tosyl-5-vinyl-4,5-dihydro-1H-benzo[b]azepin-2(3H)one (3ad)

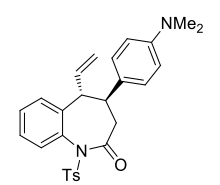

Total yield: $85 \mathrm{mg}$ (93\%); ${ }^{\mathbf{H}} \mathbf{H}$ NMR (300 MHz, $\left.\mathbf{C D C l}_{\mathbf{3}}\right) \delta 8.02(\mathrm{~d}, J=8.3 \mathrm{~Hz}, 2 \mathrm{H})$, $7.50-7.45$ (m, 1H), $7.41-7.28(\mathrm{~m}, 5 \mathrm{H}), 6.37$ (s, 4H), 5.81 (ddd, $J=17.3,10.3$, $8.1 \mathrm{~Hz}, 1 \mathrm{H}), 5.02$ (d, $J=10.6 \mathrm{~Hz}, 1 \mathrm{H}), 4.77(\mathrm{~d}, J=17.2 \mathrm{~Hz}, 1 \mathrm{H}), 3.60-3.46$ (m, $1 \mathrm{H}), 2.91(\mathrm{dd}, J=9.7,5.1 \mathrm{~Hz}, 1 \mathrm{H}), 2.81(\mathrm{~s}, 6 \mathrm{H}), 2.49-2.39(\mathrm{~m}, 4 \mathrm{H}), 2.06(\mathrm{~d}, J=12.7 \mathrm{~Hz}, 1 \mathrm{H})$. ${ }^{13}$ C NMR (75 MHz, $\left.\mathbf{C D C l}_{3}\right) \delta 170.31,149.34,145.13,139.21,136.28,136.03,135.81,132.98$, $129.91,129.28,129.15,129.06,127.91,127.22$, 126.67, 118.97, 112.40, 49.87, 46.79, 42.37, 40.50, 21.76. ATR-FTIR (cm $\left.{ }^{-1}\right): 3068,2924,1710,1614,1522,1450,1358,1167,1122,1087$, 814, 734, 708, 666; ESI-MS: calculated $\left[\mathrm{C}_{27} \mathrm{H}_{28} \mathrm{~N}_{2} \mathrm{O}_{3} \mathrm{~S}+\mathrm{Na}\right]^{+}:$483.1713, found: 483.1715; $[\alpha]^{26}{ }_{D}=+43.4\left(\mathrm{c}=2.4, \quad \mathrm{CHCl}_{3}\right)$; The product was analyzed by HPLC to determine the enantiomeric excess: $99 \%$ ee $(\mathrm{AD}-\mathrm{H}$, hexane $/ i-\mathrm{PrOH}=85 / 15$, detector: $230 \mathrm{~nm}$, flow rate: 1 $\mathrm{mL} / \mathrm{min}), \mathrm{t}_{1}($ major $)=30.3 \mathrm{~min}, \mathrm{t}_{2}($ minor $)=32.9 \mathrm{~min}$.

(4S,5R)-4-(4-chlorophenyl)-1-tosyl-5-vinyl-4,5-dihydro-1H-benzo[b]azepin-2(3H)-one (3ae)

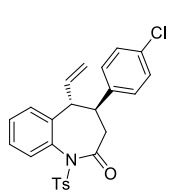
Total yield: $58 \mathrm{mg}(64 \%)$; ${ }^{1} \mathbf{H}$ NMR (300 $\left.\mathbf{M H z}, \mathbf{C D C l}_{3}\right) \delta 8.01(\mathrm{~d}, J=8.3 \mathrm{~Hz}, 2 \mathrm{H})$, $7.55-7.43(\mathrm{~m}, 1 \mathrm{H}), 7.41-7.23(\mathrm{~m}, 5 \mathrm{H}), 6.99(\mathrm{~d}, J=8.5 \mathrm{~Hz}, 2 \mathrm{H}), 6.45(\mathrm{~d}, J=8.5$ $\mathrm{Hz}, 2 \mathrm{H}), 5.90-5.71(\mathrm{~m}, 1 \mathrm{H}), 5.01(\mathrm{~d}, J=10.4 \mathrm{~Hz}, 1 \mathrm{H}), 4.73(\mathrm{~d}, J=17.2 \mathrm{~Hz}, 1 \mathrm{H})$, $3.50(\mathrm{dd}, J=11.9,8.3 \mathrm{~Hz}, 1 \mathrm{H}), 2.97(\mathrm{dd}, J=11.8,8.2 \mathrm{~Hz}, 1 \mathrm{H}), 2.59-2.37(\mathrm{~m}, 4 \mathrm{H}), 2.05(\mathrm{~d}, J=$ 
$12.9 \mathrm{~Hz}, 1 \mathrm{H}) .{ }^{13} \mathbf{C}$ NMR (75 MHz, $\left.\mathbf{C D C l}_{3}\right) \delta$ 169.87, 145.46, 140.25, 138.38, 136.13, 135.70, $135.27,132.71,129.89$, 129.35, 129.33, 129.20, 128.70, 128.60, 127.58, 126.63, 119.43, 110.00, 49.98, 46.71, 41.72, 21.78. ATR-FTIR ( cm$\left.^{-1}\right):$ 3073, 2923, 1709, 1492, 1365, 1170, 1123, 1088, 1014, 925, 810, 735, 687; ESI-MS: calculated $\left[\mathrm{C}_{25} \mathrm{H}_{22} \mathrm{ClNO}_{3} \mathrm{~S}+\mathrm{Na}\right]^{+}$: 474.0901, found: $474.0914 ;[\alpha]^{26} \mathbf{D}=+19.4\left(\mathrm{c}=1.6, \mathrm{CHCl}_{3}\right)$; The product was analyzed by HPLC to determine the enantiomeric excess: 97\% ee (AD-H, hexane/i-PrOH $=85 / 15$, detector: $254 \mathrm{~nm}$, flow rate: 1 $\mathrm{mL} / \mathrm{min}), \mathrm{t}_{1}($ major $)=26.0 \mathrm{~min}, \mathrm{t}_{2}($ minor $)=28.0 \mathrm{~min}$.

(4S,5R)-4-(4-fluorophenyl)-1-tosyl-5-vinyl-4,5-dihydro-1H-benzo[b]azepin-2(3H)-one (3af) Total yield: $67 \mathrm{mg}(77 \%)$; ${ }^{1} \mathbf{H}$ NMR (300 MHz, $\left.\mathbf{C D C l}_{3}\right) \delta 8.01(\mathrm{~d}, J=8.4 \mathrm{~Hz}, 2 \mathrm{H})$, $7.55-7.42(\mathrm{~m}, 1 \mathrm{H}), 7.43-7.23(\mathrm{~m}, 5 \mathrm{H}), 6.77-6.61(\mathrm{~m}, 2 \mathrm{H}), 6.59-6.42(\mathrm{~m}, 2 \mathrm{H})$, $5.80(\mathrm{ddd}, J=17.2,10.3,8.2 \mathrm{~Hz}, 1 \mathrm{H}), 5.01(\mathrm{~d}, J=10.4 \mathrm{~Hz}, 1 \mathrm{H}), 4.73(\mathrm{~d}, J=17.2$ $\mathrm{Hz}, 1 \mathrm{H}), 3.50(\mathrm{dd}, J=11.8,8.3 \mathrm{~Hz}, 1 \mathrm{H}), 2.98(\mathrm{dd}, J=11.9,7.9 \mathrm{~Hz}, 1 \mathrm{H}), 2.55-2.39(\mathrm{~m}, 4 \mathrm{H})$, $2.06(\mathrm{dd}, J=12.9,0.9 \mathrm{~Hz}, 1 \mathrm{H}) .{ }^{13} \mathbf{C}$ NMR (75 MHz, $\left.\mathbf{C D C l}_{3}\right) \delta 169.99,161.64(\mathrm{~d}, J=245.5 \mathrm{~Hz})$, 160.01, 145.41, 138.52, 137.52 (d, $J=3.3 \mathrm{~Hz}), 135.95$ (d, $J=33.8 \mathrm{~Hz}), 135.42,129.90,129.31$, 129.17, 128.84 (d, $J=7.9 \mathrm{~Hz}), 127.53,126.62,119.31,115.26$ (d, $J=21.3 \mathrm{~Hz}), 49.88,46.98$, 41.85, 21.76. ATR-FTIR (cm $\left.{ }^{-1}\right): 3073,2982,1824,1709,1511,1385,1223,1171,1123,1087$, 908, 824, 765, 728, 658; ESI-MS: calculated $\left[\mathrm{C}_{25} \mathrm{H}_{22} \mathrm{FNO}_{3} \mathrm{~S}+\mathrm{Na}^{+}\right.$: 458.1197, found: 458.1200; $[\alpha]^{26}{ }_{D}=+4.4\left(\mathrm{c}=2.2, \quad \mathrm{CHCl}_{3}\right)$; The product was analyzed by HPLC to determine the enantiomeric excess: $98 \%$ ee $(\mathrm{AD}-\mathrm{H}$, hexane $/ i-\mathrm{PrOH}=85 / 15$, detector: $230 \mathrm{~nm}$, flow rate: 1 $\mathrm{mL} / \mathrm{min}), \mathrm{t}_{1}($ minor $)=24.4 \mathrm{~min}, \mathrm{t}_{2}($ major $)=26.1 \mathrm{~min}$.

\section{(4S,5R)-4-(4-nitrophenyl)-1-tosyl-5-vinyl-4,5-dihydro-1H-benzo[b]azepin-2(3H)-one (3ag)}

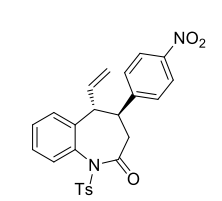

Total yield: 90 mg (98\%); ${ }^{1} \mathbf{H}$ NMR (400 $\mathbf{~ M H z , ~} \mathbf{C D C l}_{3}$ ) $\delta 8.03$ (dd, $J=12.3,5.8$ $\mathrm{Hz}, 2 \mathrm{H}), 7.94-7.84(\mathrm{~m}, 2 \mathrm{H}), 7.54-7.46(\mathrm{~m}, 1 \mathrm{H}), 7.38(\mathrm{dt}, J=7.4,3.1 \mathrm{~Hz}, 4 \mathrm{H})$, $7.30(\mathrm{dq}, J=7.4,3.7 \mathrm{~Hz}, 1 \mathrm{H}), 6.72(\mathrm{~d}, J=8.7 \mathrm{~Hz}, 2 \mathrm{H}), 5.82(\mathrm{ddd}, J=17.2,10.3$, $8.3 \mathrm{~Hz}, 1 \mathrm{H}), 5.01(\mathrm{~d}, J=10.4 \mathrm{~Hz}, 1 \mathrm{H}), 4.71(\mathrm{~d}, J=17.2 \mathrm{~Hz}, 1 \mathrm{H}), 3.55$ (dd, $J=11.9,8.4 \mathrm{~Hz}, 1 \mathrm{H})$, $3.11(\mathrm{dd}, J=11.9,8.0 \mathrm{~Hz}, 1 \mathrm{H}), 2.58-2.50(\mathrm{~m}, 1 \mathrm{H}), 2.48(\mathrm{~s}, 3 \mathrm{H}), 2.11-2.05(\mathrm{~m}, 1 \mathrm{H}) .{ }^{13} \mathbf{C} \mathbf{~ N M R}$ (101 MHz, $\left.\mathbf{C D C l}_{3}\right) \delta 169.61,149.26,146.89,145.71,137.79,136.06,135.64,134.76,129.88$, $129.54,129.43$, 129.28, 128.39, 127.87, 126.64, 123.72, 119.81, 50.22, 46.77, 41.19, 21.80 . ATR-FTIR (cm $\left.{ }^{-1}\right):$ 3076, 2923, 1703, 1520, 1347, 1171, 1088, 908, 731, 687, 649; ESI-MS: calculated $\left[\mathrm{C}_{25} \mathrm{H}_{22} \mathrm{~N}_{2} \mathrm{O}_{5} \mathrm{~S}+\mathrm{Na}\right]^{+}: 485.1142$, found: 485.1130; $[\alpha]^{\mathbf{2 6}} \mathbf{D}=+18.5\left(\mathrm{c}=1.9, \mathrm{CHCl}_{3}\right)$; The product was analyzed by HPLC to determine the enantiomeric excess: $91 \%$ ee (AD-H, hexane $/ i-\mathrm{PrOH}=70 / 30$, detector: $230 \mathrm{~nm}$, flow rate: $1 \mathrm{~mL} / \mathrm{min}), \mathrm{t}_{1}($ minor $)=24.5 \mathrm{~min}, \mathrm{t}_{2}$ (major) $=$ $35.8 \mathrm{~min}$. 
(4S,5R)-4-(2-methoxyphenyl)-1-tosyl-5-vinyl-4,5-dihydro-1H-benzo[b]azepin-2(3H)-one

(3ah)

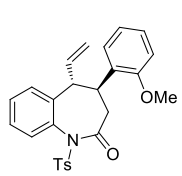

Total yield: $78 \mathrm{mg}(87 \%)$; ${ }^{\mathbf{1}} \mathbf{H}$ NMR (400 MHz, $\left.\mathbf{C D C l}_{3}\right) \delta 8.00(\mathrm{~d}, J=8.3 \mathrm{~Hz}, 2 \mathrm{H})$, $7.50-7.42(\mathrm{~m}, 1 \mathrm{H}), 7.37-7.28(\mathrm{~m}, 5 \mathrm{H}), 7.07-7.00(\mathrm{~m}, 1 \mathrm{H}), 6.69(\mathrm{~d}, J=7.7 \mathrm{~Hz}$,

$1 \mathrm{H}), 6.56-6.44(\mathrm{~m}, 1 \mathrm{H}), 6.11-5.89(\mathrm{~m}, 1 \mathrm{H}), 5.78(\mathrm{ddd}, J=17.5,10.4,7.3 \mathrm{~Hz}$, 1H), $5.01(\mathrm{~d}, J=10.5 \mathrm{~Hz}, 1 \mathrm{H}), 4.85-4.74(\mathrm{~m}, 1 \mathrm{H}), 3.73-3.55(\mathrm{~m}, 5 \mathrm{H}), 2.45(\mathrm{~s}, 3 \mathrm{H}), 2.40(\mathrm{t}, J=$ $6.4 \mathrm{~Hz}, 1 \mathrm{H}), 1.98(\mathrm{~d}, J=12.7 \mathrm{~Hz}, 1 \mathrm{H}) .{ }^{13} \mathbf{C}$ NMR (101 MHz, $\left.\mathbf{C D C l}_{\mathbf{3}}\right) \delta$ 170.38, 156.22, 145.20, 136.37, 135.97, 135.80, 129.87, 129.44, 129.28, 129.19, 128.17, 127.73, 127.31, 126.98, 126.60, 120.28, 118.67, 110.25, 55.27, 45.96, 45.10, 40.98, 21.76. ATR-FTIR (cm $\left.{ }^{-1}\right):$ 2924, 1710, 1492, 1383, 1241, 1170, 1105, 1028, 908, 813, 753, 707, 660; ESI-MS: calculated $\left[\mathrm{C}_{26} \mathrm{H}_{25} \mathrm{NO}_{4} \mathrm{~S}+\mathrm{Na}^{+}\right.$: 470.1397, found: $470.1388 ;[\alpha]^{26} \mathrm{D}=+16.6\left(\mathrm{c}=2.1, \mathrm{CHCl}_{3}\right)$; The product was analyzed by HPLC to determine the enantiomeric excess: $98 \%$ ee $(\mathrm{AD}-\mathrm{H}$, hexane $/ i-\mathrm{PrOH}=70 / 30$, detector: $230 \mathrm{~nm}$, flow rate: $1 \mathrm{~mL} / \mathrm{min}), \mathrm{t}_{1}($ major $)=7.7 \mathrm{~min}, \mathrm{t}_{2}($ minor $)=8.6 \mathrm{~min}$.

(4S,5R)-4-(3-chlorophenyl)-1-tosyl-5-vinyl-4,5-dihydro-1H-benzo[b]azepin-2(3H)-one (3ai) Total yield: 54 mg (60\%); ${ }^{\mathbf{1}} \mathbf{H}$ NMR (300 $\left.\mathbf{M H z}, \mathbf{C D C l}_{3}\right) \delta 7.99(\mathrm{~d}, J=8.4 \mathrm{~Hz}, 2 \mathrm{H})$, $7.54-7.48(\mathrm{~m}, 1 \mathrm{H}), 7.43-7.25(\mathrm{~m}, 5 \mathrm{H}), 7.08-7.01(\mathrm{~m}, 1 \mathrm{H}), 6.96(\mathrm{t}, J=8.0 \mathrm{~Hz}$, $1 \mathrm{H}), 6.44(\mathrm{t}, J=4.1 \mathrm{~Hz}, 2 \mathrm{H}), 5.89-5.71(\mathrm{~m}, 1 \mathrm{H}), 5.02(\mathrm{~d}, J=10.5 \mathrm{~Hz}, 1 \mathrm{H}), 4.72$ $(\mathrm{d}, J=17.2 \mathrm{~Hz}, 1 \mathrm{H}), 3.43(\mathrm{dd}, J=12.0,8.3 \mathrm{~Hz}, 1 \mathrm{H}), 2.95(\mathrm{dd}, J=11.9,8.3 \mathrm{~Hz}, 1 \mathrm{H}), 2.57-2.37$ $(\mathrm{m}, 4 \mathrm{H}), 2.09(\mathrm{~d}, J=12.9 \mathrm{~Hz}, 1 \mathrm{H}) .{ }^{13} \mathbf{C} \mathbf{N M R}\left(\mathbf{7 5} \mathbf{~ M H z}, \mathbf{C D C l}_{3}\right) \delta 169.73,145.63,143.74,138.19$, 135.96, 135.66, 135.19, 134.29, 129.70, 129.52, 129.36, 127.59, 127.34, 127.29, 126.60, 125.73, 119.51, 50.30, 46.42, 41.57, 21.85. ATR-FTIR ( cm$\left.^{-1}\right): 3067,2826,1710,1596,1431,1386$, 1171, 1087, 996, 911, 788, 736, 694, 604; ESI-MS: calculated $\left[\mathrm{C}_{25} \mathrm{H}_{22} \mathrm{ClNO}_{3} \mathrm{~S}+\mathrm{Na}\right]^{+}:$44.0901, found: $474.0912 ;[\alpha]^{26}{ }_{\mathbf{D}}=+12.5\left(\mathrm{c}=1.1, \mathrm{CHCl}_{3}\right)$; The product was analyzed by HPLC to determine the enantiomeric excess: 95\% ee $(\mathrm{OD}-\mathrm{H}$, hexane $/ i-\mathrm{PrOH}=80 / 20$, detector: $230 \mathrm{~nm}$, flow rate: $1 \mathrm{~mL} / \mathrm{min}), \mathrm{t}_{1}($ minor $)=10.1 \mathrm{~min}, \mathrm{t}_{2}($ major $)=12.6 \mathrm{~min}$.

(4S,5R)-4-(4-hydroxy-3-methoxyphenyl)-1-tosyl-5-vinyl-4,5-dihydro-1H-benzo[b]azepin2(3H)-one (3aj)

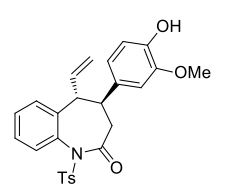

Total yield: $64 \mathrm{mg}(69 \%) ;{ }^{1} \mathbf{H}$ NMR (400 $\left.\mathbf{~ M H z ,} \mathbf{C D C l}_{3}\right) \delta 8.00(\mathrm{~d}, J=8.4 \mathrm{~Hz}$, 2H), $7.48-7.43(\mathrm{~m}, 1 \mathrm{H}), 7.38-7.29(\mathrm{~m}, 5 \mathrm{H}), 6.60-6.54(\mathrm{~m}, 1 \mathrm{H}), 6.43(\mathrm{~d}, J=$ $2.0 \mathrm{~Hz}, 1 \mathrm{H}), 6.10-6.04(\mathrm{~m}, 1 \mathrm{H}), 5.84(\mathrm{ddd}, J=17.2,10.3,8.2 \mathrm{~Hz}, 1 \mathrm{H}), 5.41$ (s, 1H), 5.02 (dd, $J=10.4,0.6 \mathrm{~Hz}, 1 \mathrm{H}), 4.84-4.74(\mathrm{~m}, 1 \mathrm{H}), 3.66$ (s, 3H), 3.57 (dd, $J=12.6,7.6 \mathrm{~Hz}$, $1 \mathrm{H}), 2.93(\mathrm{dd}, J=12.0,7.9 \mathrm{~Hz}, 1 \mathrm{H}), 2.48(\mathrm{dd}, J=12.9,8.4 \mathrm{~Hz}, 1 \mathrm{H}), 2.42(\mathrm{~s}, 3 \mathrm{H}), 2.12(\mathrm{dd}, J=$ 
12.9, $0.9 \mathrm{~Hz}, 1 \mathrm{H}) .{ }^{\mathbf{1 3}} \mathbf{C}$ NMR (101 MHz, $\left.\mathbf{C D C l}_{3}\right) \delta$ 170.60, 146.61, 145.20, 144.47, 139.11, $136.58,135.73,133.99,129.66,129.34,129.29,129.25,128.81,127.39,126.61,120.38,119.10$, 113.94, 109.62, 55.82, 50.61, 47.26, 42.16, 21.73. ATR-FTIR (cm-1): 2924, 1707, 1599, 1518, 1382, 1168, 1123, 1034, 910, 720, 649; ESI-MS: calculated $\left[\mathrm{C}_{26} \mathrm{H}_{25} \mathrm{NO}_{5} \mathrm{~S}+\mathrm{Na}\right]^{+}: 486.1346$, found: $486.1339 ;[\alpha]^{26} \mathbf{D}=+7.5\left(\mathrm{c}=1.8, \mathrm{CHCl}_{3}\right)$; The product was analyzed by HPLC to determine the enantiomeric excess: $98 \%$ ee $(\mathrm{AD}-\mathrm{H}$, hexane $/ i-\mathrm{PrOH}=70 / 30$, detector: $230 \mathrm{~nm}$, flow rate: $1 \mathrm{~mL} / \mathrm{min}), \mathrm{t}_{1}($ major $)=29.5 \mathrm{~min}, \mathrm{t}_{2}($ minor $)=39.6 \mathrm{~min}$.

\section{(4S,5R)-4-(furan-2-yl)-1-tosyl-5-vinyl-4,5-dihydro-1H-benzo[b]azepin-2(3H)-one (3ak)}

Total yield: $49 \mathrm{mg}(80 \%) ;{ }^{1} \mathbf{H}$ NMR (300 MHz, $\left.\mathbf{C D C l}_{3}\right) \delta 7.95(\mathrm{~d}, J=8.4 \mathrm{~Hz}, 2 \mathrm{H})$, $7.47(\mathrm{dt}, J=7.3,3.8 \mathrm{~Hz}, 1 \mathrm{H}), 7.39-7.25(\mathrm{~m}, 5 \mathrm{H}), 7.06-7.01(\mathrm{~m}, 1 \mathrm{H}), 6.08(\mathrm{dd}, J$ $=3.2,1.8 \mathrm{~Hz}, 1 \mathrm{H}), 5.98-5.82(\mathrm{~m}, 1 \mathrm{H}), 5.49(\mathrm{~d}, J=3.2 \mathrm{~Hz}, 1 \mathrm{H}), 5.13(\mathrm{~d}, J=10.4$

$\mathrm{Hz}, 1 \mathrm{H}), 4.87(\mathrm{~d}, J=17.2 \mathrm{~Hz}, 1 \mathrm{H}), 3.50$ (dd, $J=11.9,7.9 \mathrm{~Hz}, 1 \mathrm{H}), 3.14$ (dd, $J=11.9,6.6 \mathrm{~Hz}$, $1 \mathrm{H}), 2.46-2.36(\mathrm{~m}, 4 \mathrm{H}), 2.24(\mathrm{dd}, J=12.8,1.4 \mathrm{~Hz}, 1 \mathrm{H}) .{ }^{13} \mathbf{C} \mathbf{N M R}\left(\mathbf{7 5} \mathbf{~ M H z}, \mathbf{C D C l}_{3}\right) \delta 169.28$, $154.69,145.15,141.13,137.87,136.28,135.90,135.54,129.65,129.30,129.23,129.20,127.62$, 126.72, 118.91, 110.15, 105.83, 45.41, 43.43, 40.23, 21.71. ATR-FTIR (cm $\left.{ }^{-1}\right): 3073,2925,1713$, 1597, 1383, 1170, 1211, 1007, 926, 815, 736, 687; ESI-MS: calculated $\left[\mathrm{C}_{23} \mathrm{H}_{21} \mathrm{NO}_{4} \mathrm{~S}+\mathrm{Na}\right]^{+}$: 430.1083, found: $430.1081 ;[\boldsymbol{\alpha}]^{26} \mathrm{D}=+29.7\left(\mathrm{c}=1.5, \mathrm{CHCl}_{3}\right)$; The product was analyzed by HPLC to determine the enantiomeric excess: $98 \%$ ee $(\mathrm{AD}-\mathrm{H}$, hexane $/ i-\mathrm{PrOH}=70 / 30$, detector: $230 \mathrm{~nm}$, flow rate: $1 \mathrm{~mL} / \mathrm{min}), \mathrm{t}_{1}($ major $)=9.9 \mathrm{~min}, \mathrm{t}_{2}($ minor $)=13.0 \mathrm{~min}$.

\section{(4R,5R)-4-((E)-prop-1-en-1-yl)-1-tosyl-5-vinyl-4,5-dihydro-1H-benzo[b]azepin-2(3H)-one} (3al)

Total yield: $60 \mathrm{mg}(79 \%) ;{ }^{1} \mathbf{H} \mathbf{N M R}\left(\mathbf{4 0 0} \mathbf{M H z}, \mathbf{C D C l}_{3}\right) \delta 7.91(\mathrm{~d}, J=8.3 \mathrm{~Hz}, 2 \mathrm{H})$,

$=1.46-7.41(\mathrm{~m}, 1 \mathrm{H}), 7.30(\mathrm{dt}, J=6.3,2.5 \mathrm{~Hz}, 4 \mathrm{H}), 7.24-7.20(\mathrm{~m}, 1 \mathrm{H}), 5.82(\mathrm{ddd}, J$ $4.91-4.85$ (m, 1H), 2.97 (dd, $J=10.9,8.4 \mathrm{~Hz}, 1 \mathrm{H}), 2.40$ (s, 3H), $2.38-2.33$ (m, 1H), 2.30 (dd, $J$ $=12.3,7.5 \mathrm{~Hz}, 1 \mathrm{H}), 2.08-1.99(\mathrm{~m}, 1 \mathrm{H}), 1.48(\mathrm{dd}, J=6.4,1.4 \mathrm{~Hz}, 3 \mathrm{H}) .{ }^{13} \mathbf{C}$ NMR $(\mathbf{1 0 1} \mathbf{M H z}$, $\left.\mathbf{C D C l}_{3}\right) \delta 170.47,145.10,138.80,136.40,136.37,135.69,131.46,129.33,129.28,129.09$, 129.00, 127.19, 127.09, 126.51, 118.73, 47.63, 46.69, 41.04, 21.70, 17.76. ATR-FTIR (cm $\left.{ }^{-1}\right)$ : 2923, 2854, 1710, 1597, 1484, 1451, 1364, 1166, 1086, 815, 707, 666; ESI-MS: calculated $\left[\mathrm{C}_{22} \mathrm{H}_{23} \mathrm{NO}_{3} \mathrm{~S}+\mathrm{Na}\right]^{+}:$404.1291, found: 404.1295; The product was analyzed by HPLC to determine the enantiomeric excess: $92 \%$ ee $(\mathrm{AS}-\mathrm{H}$, hexane $/ i-\mathrm{PrOH}=90 / 10$, detector: $254 \mathrm{~nm}$, flow rate: $1 \mathrm{~mL} / \mathrm{min}), \mathrm{t}_{1}($ minor $)=10.8 \mathrm{~min}, \mathrm{t}_{2}($ major $)=14.6 \mathrm{~min}$. 
(4S,5R)-4-methyl-1-tosyl-5-vinyl-4,5-dihydro-1H-benzo[b]azepin-2(3H)-one (3am)

Total yield: $29 \mathrm{mg}(42 \%) ;{ }^{1} \mathbf{H}$ NMR (300 MHz, $\left.\mathbf{C D C l}_{3}\right) \delta 7.90(\mathrm{~d}, J=8.4 \mathrm{~Hz}, 2 \mathrm{H})$, $7.44(\mathrm{dd}, J=5.5,3.7 \mathrm{~Hz}, 1 \mathrm{H}), 7.31-7.25(\mathrm{~m}, 5 \mathrm{H}), 5.89(\mathrm{ddd}, J=17.1,10.2,8.7 \mathrm{~Hz}$, $1 \mathrm{H}), 5.27-5.18(\mathrm{~m}, 1 \mathrm{H}), 4.93(\mathrm{~d}, J=17.1 \mathrm{~Hz}, 1 \mathrm{H}), 2.70(\mathrm{dd}, J=11.0,8.8 \mathrm{~Hz}, 1 \mathrm{H})$, $2.40(\mathrm{~s}, 3 \mathrm{H}), 2.33-2.23(\mathrm{~m}, 2 \mathrm{H}), 1.96-1.89(\mathrm{~m}, 1 \mathrm{H}), 0.83(\mathrm{~d},, J=6.7 \mathrm{~Hz}, 3 \mathrm{H}) .{ }^{13} \mathbf{C}$ NMR $(\mathbf{7 5}$ MHz, $\left.\mathbf{C D C l}_{3}\right) \delta 170.66,145.11,136.62,129.36,129.26,129.09,127.11,126.11,118.74,110.03$, 106.26, 49.13, 41.45, 39.28, 21.73, 19.43. ATR-FTIR (cm $\left.{ }^{-1}\right): 2961,1768,1713,1598,1454$, 1365, 1237, 1166, 1090, 927, 815, 763, 667; ESI-MS: calculated $\left[\mathrm{C}_{20} \mathrm{H}_{21} \mathrm{NO}_{3} \mathrm{~S}+\mathrm{Na}\right]^{+}: 378.1134$, found: 378.1140 ; The product was analyzed by HPLC to determine the enantiomeric excess: $84 \%$ $e e(\mathrm{IA}-\mathrm{H}$, hexane $/ i-\mathrm{PrOH}=90 / 10$, detector: $230 \mathrm{~nm}$, flow rate: $1 \mathrm{~mL} / \mathrm{min}), \mathrm{t}_{1}($ major $)=12.5 \mathrm{~min}$, $\mathrm{t}_{2}($ minor $)=13.7 \mathrm{~min}$.

(4S,5R)-8-methyl-4-phenyl-1-tosyl-5-vinyl-1,3,4,5-tetrahydro-2H-benzo[b]azepin-2-one (3ba)

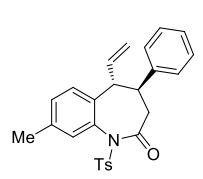

Total yield: $76 \mathrm{mg}(88 \%)$; ${ }^{1} \mathbf{H}$ NMR (300 MHz, $\left.\mathbf{C D C l}_{3}\right) \delta 8.11-8.07(\mathrm{~m}, 2 \mathrm{H})$, $7.43(\mathrm{~d}, J=8.1 \mathrm{~Hz}, 2 \mathrm{H}), 7.37(\mathrm{~s}, 1 \mathrm{H}), 7.25-7.23(\mathrm{~m}, 2 \mathrm{H}), 7.16-7.04(\mathrm{~m}, 3 \mathrm{H})$, $6.55-6.51(\mathrm{~m}, 2 \mathrm{H}), 5.87$ (ddd, $J=17.1,10.4,8.1,1 \mathrm{H}), 5.07-5.03(\mathrm{~m}, 1 \mathrm{H}), 4.82$ - $4.76(\mathrm{~m}, 1 \mathrm{H}), 3.57(\mathrm{dd}, J=12.0,8.1 \mathrm{~Hz}, 1 \mathrm{H}), 3.02(\mathrm{dd}, J=12.0,8.2 \mathrm{~Hz}, 1 \mathrm{H}), 2.59-2.52(\mathrm{~m}$, 4H), 2.44 (s, 3H), 2.15 (dd, $J=12.8,1.3 \mathrm{~Hz}, 1 \mathrm{H}) ;{ }^{13} \mathbf{C}$ NMR (75 MHz, CDCl 3 ) $\delta$ 170.28, 145.40, 141.93, 137.52, 136.30, 135.90, 135.71, 135.63, 130.16, 130.06, 129.86, 129.38, 128.47, 127.44, 127.02, 126.47, 119.03, 50.89, 46.50, 42.01, 21.89, 21.14. ATR-FTIR $\left(\mathbf{c m}^{-1}\right):$ 3028. 2982, 2924 , $1705,1597,1497,1454,1408,1362,1261,1234,1184,1169,1130,1084,922,845,814,748$, 698, 687, 656, 610, 579, 548; ESI-MS: calculated $\left[\mathrm{C}_{26} \mathrm{H}_{25} \mathrm{NO}_{3} \mathrm{~S}+\mathrm{Na}\right]^{+}:$454.1447, found: $454.1442 ;[\alpha]^{26} \mathrm{D}=+50.3\left(\mathrm{c}=0.6, \mathrm{CHCl}_{3}\right)$; The product was analyzed by HPLC to determine the enantiomeric excess: 99\% ee $(\mathrm{AD}-\mathrm{H}$, hexane/i-PrOH $=70 / 30$, detector: $230 \mathrm{~nm}$, flow rate: 1 $\mathrm{mL} / \mathrm{min}), \mathrm{t}_{1}($ minor $)=11.5$ min, $\mathrm{t}_{2}($ major $)=16.5 \mathrm{~min}$.

\section{(4S,5R)-8-chloro-4-phenyl-1-tosyl-5-vinyl-4,5-dihydro-1H-benzo[b]azepin-2(3H)-one (3ca)}

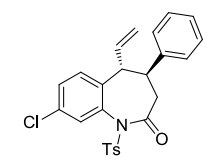

Total yield: $54 \mathrm{mg}(61 \%)$; ${ }^{1} \mathbf{H}$ NMR (400 $\left.\mathbf{M H z}, \mathbf{C D C l}_{3}\right) \delta 8.02(\mathrm{dd}, J=7.2,5.6$ $\mathrm{Hz}, 2 \mathrm{H}), 7.49$ (d, $J=2.1 \mathrm{~Hz}, 1 \mathrm{H}), 7.40-7.31(\mathrm{~m}, 3 \mathrm{H}), 7.24(\mathrm{~d}, J=8.4 \mathrm{~Hz}, 1 \mathrm{H})$, $7.09-7.00(\mathrm{~m}, 3 \mathrm{H}), 6.55-6.43(\mathrm{~m}, 2 \mathrm{H}), 5.76(\mathrm{ddd}, J=17.2,10.4,8.1 \mathrm{~Hz}, 1 \mathrm{H})$, $5.01(\mathrm{~d}, J=10.5 \mathrm{~Hz}, 1 \mathrm{H}), 4.77(\mathrm{dd}, J=16.1,9.8 \mathrm{~Hz}, 1 \mathrm{H}), 3.53(\mathrm{dd}, J=12.0,8.1 \mathrm{~Hz}, 1 \mathrm{H}), 2.97$ $(\mathrm{dd}, J=11.9,7.8 \mathrm{~Hz}, 1 \mathrm{H}), 2.50-2.41(\mathrm{~m}, 4 \mathrm{H}), 2.12(\mathrm{dd}, J=13.0,1.0 \mathrm{~Hz}, 1 \mathrm{H}) .{ }^{13} \mathbf{C}$ NMR $(\mathbf{1 0 1}$ MHz, $\left.\mathbf{C D C l}_{3}\right) \delta 169.57,145.61,141.38,137.41,136.64,135.93,135.13,132.88,129.88,129.41$, 129.37, 129.20, 128.48, 127.70, 127.28, 127.11, 119.63, 50.47, 46.29, 41.76, 21.77. ATR-FTIR $\left(\mathbf{c m}^{-1}\right):$ 3068, 2925, 1713, 1580, 1403, 1365, 1260, 1223, 1171, 1087, 999 908, 727, 665; ESI- 
MS: calculated $\left[\mathrm{C}_{25} \mathrm{H}_{22} \mathrm{ClNO}_{3} \mathrm{~S}+\mathrm{Na}\right]^{+}: 474.0901$, found: 474.0908; $[\alpha]^{26}{ }_{\mathrm{D}}=-1.9\left(\mathrm{c}=1.4, \mathrm{CHCl}_{3}\right)$; The product was analyzed by HPLC to determine the enantiomeric excess: $92 \%$ ee (AD-H, hexane $/ i-\mathrm{PrOH}=70 / 30$, detector: $230 \mathrm{~nm}$, flow rate: $1 \mathrm{~mL} / \mathrm{min}), \mathrm{t}_{1}($ minor $)=9.6 \mathrm{~min}, \mathrm{t}_{2}($ major $)=$ $14.8 \mathrm{~min}$.

(4S,5R)-8-fluoro-4-phenyl-1-tosyl-5-vinyl-1,3,4,5-tetrahydro-2H-benzo[b]azepin-2-one (3da)

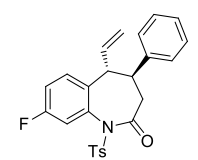
Total yield: 54 mg (61\%); ${ }^{1} \mathbf{H}$ NMR (300 MHz, $\left.\mathbf{C D C l}_{3}\right) \delta 8.11-8.06(\mathrm{~m}, 2 \mathrm{H})$, $7.49-7.40$ (m, 2H), $7.38-7.28$ (m, 2H), $7.19-7.06(\mathrm{~m}, 4 \mathrm{H}), 6.61-6.55(\mathrm{~m}, 2 \mathrm{H})$, 5.85 (ddd, $J=17.2,10.4,8.0 \mathrm{~Hz}, 1 \mathrm{H}), 5.14-5.03(\mathrm{~m}, 1 \mathrm{H}), 4.89-4.76(\mathrm{~m}, 1 \mathrm{H})$, $3.60(\mathrm{dd}, J=12.0,7.9 \mathrm{~Hz}, 1 \mathrm{H}), 3.05(\mathrm{dd}, J=11.7,7.4 \mathrm{~Hz}, 1 \mathrm{H}), 2.60-2.50(\mathrm{~m}, 4 \mathrm{H}), 2.19$ (dd, $J=13.0,1.3 \mathrm{~Hz}, 1 \mathrm{H}) ;{ }^{13} \mathbf{C} \mathbf{N M R}\left(75 \mathrm{MHz}, \mathrm{CDCl}_{3}\right): \delta 169.68,161.07(\mathrm{~d}, J=248.0 \mathrm{~Hz}), 145.61$, $141.47,136.70$ (d, $J=10.2 \mathrm{~Hz}), 136.91,135.36,134.73$ (d, $J=3.6 \mathrm{~Hz}), 129.87,129.41,128.47$, $127.74(\mathrm{~d}, J=8.9 \mathrm{~Hz}), 127.28,127.08,119.49,116.63(\mathrm{~d}, J=23.7 \mathrm{~Hz}), 116.23(\mathrm{~d}, J=20.8 \mathrm{~Hz})$, $50.61(\mathrm{~d}, J=1.3 \mathrm{~Hz}), 46.12$, 41.82, 21.79; ATR-FTIR $\left(\mathbf{c m}^{-1}\right):$ 3854, 3734, 3649, 2982, 2920, 1717, 1709, 1593, 1559, 1543, 1508, 1497, 1489, 1474, 1458, 1362, 1123, 1084, 814, 779, 702, 683, 656, 584, 552; ESI-MS: calculated $\left[\mathrm{C}_{25} \mathrm{H}_{22} \mathrm{FNO}_{3} \mathrm{~S}+\mathrm{Na}^{+}:\right.$458.1197, found: 458.1202; $[\boldsymbol{\alpha}]^{\mathbf{2 6}} \mathrm{D}=+17.9\left(\mathrm{c}=0.9, \mathrm{CHCl}_{3}\right)$; The product was analyzed by HPLC to determine the enantiomeric excess: $93 \%$ ee $(\mathrm{AD}-\mathrm{H}$, hexane/i-PrOH $=70 / 30$, detector: $230 \mathrm{~nm}$, flow rate: 1 $\mathrm{mL} / \mathrm{min}), \mathrm{t}_{1}(\operatorname{minor})=9.2 \mathrm{~min}, \mathrm{t}_{2}($ major $)=12.7 \mathrm{~min}$.

(4S,5R)-8-bromo-4-phenyl-1-tosyl-5-vinyl-4,5-dihydro-1H-benzo[b]azepin-2(3H)-one (3ea)

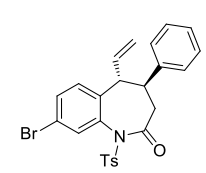
Total yield: $54 \mathrm{mg}(55 \%) ;{ }^{1} \mathbf{H}$ NMR (400 MHz, $\left.\mathbf{C D C l}_{3}\right) \delta 8.01(\mathrm{~d}, J=8.4 \mathrm{~Hz}$, 2H), $7.64(\mathrm{~d}, J=2.0 \mathrm{~Hz}, 1 \mathrm{H}), 7.51-7.45(\mathrm{~m}, 1 \mathrm{H}), 7.37$ (d, $J=8.2 \mathrm{~Hz}, 2 \mathrm{H}), 7.19$ - $7.15(\mathrm{~m}, 1 \mathrm{H}), 7.10-6.97(\mathrm{~m}, 3 \mathrm{H}), 6.53-6.45$ (m, 2H), 5.76 (ddd, $J=17.3$, $10.3,8.1 \mathrm{~Hz}, 1 \mathrm{H}), 5.00(\mathrm{dd}, J=9.6,5.8 \mathrm{~Hz}, 1 \mathrm{H}), 4.76(\mathrm{dd}, J=16.2,10.3 \mathrm{~Hz}, 1 \mathrm{H}), 3.51(\mathrm{dd}, J=$ 12.0, 8.1 Hz, 1H), 2.97 (dd, $J=12.0,8.0 \mathrm{~Hz}, 1 \mathrm{H}), 2.53-2.42(\mathrm{~m}, 4 \mathrm{H}), 2.12$ (dd, $J=13.0,0.8 \mathrm{~Hz}$, 1H). ${ }^{13} \mathbf{C}$ NMR (101 MHz, $\left.\mathbf{C D C l}_{3}\right) \delta$ 169.54, 145.61, 141.36, 137.92, 136.80, 135.93, 135.06, $132.30,132.01,129.88,129.41,128.48,128.03,127.27,127.11,120.49,119.66,50.39,46.36$, 41.74, 21.78. ATR-FTIR (cm-1): 3066, 2923, 1713, 1592, 1304, 1261, 1170, 1127, 1086, 994, 909, 814, 731, 655; ESI-MS: calculated $\left[\mathrm{C}_{25} \mathrm{H}_{22} \mathrm{BrNO}_{3} \mathrm{~S}+\mathrm{Na}\right]^{+}:$518.0396, found: 518.0402; $[\boldsymbol{\alpha}]^{\mathbf{2 6}}{ }_{\mathrm{D}}=-4.9\left(\mathrm{c}=1.5, \mathrm{CHCl}_{3}\right)$; The product was analyzed by HPLC to determine the enantiomeric excess: $93 \%$ ee $(\mathrm{AD}-\mathrm{H}$, hexane $/ \mathrm{i}-\mathrm{PrOH}=70 / 30$, detector: $230 \mathrm{~nm}$, flow rate: $1 \mathrm{~mL} / \mathrm{min}), \mathrm{t}_{1}$ (minor) $=10.7$ min, $\mathrm{t}_{2}($ major $)=16.9 \min$. 
(4S,5R)-4-phenyl-1-tosyl-8-(trifluoromethyl)-5-vinyl-1,3,4,5-tetrahydro-2H-benzo[b]azepin2-one (3fa)

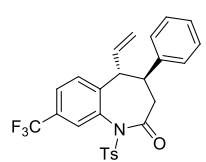

Total yield: $42 \mathrm{mg}(44 \%) ;{ }^{1} \mathbf{H}$ NMR (600 MHz, $\left.\mathbf{C D C l}_{3}\right) \delta 8.09(\mathrm{~d}, J=8.1 \mathrm{~Hz}$, 2H), $7.81(\mathrm{~s}, 1 \mathrm{H}), 7.68(\mathrm{~d}, J=8.2 \mathrm{~Hz}, 1 \mathrm{H}), 7.52(\mathrm{~d}, J=8.1 \mathrm{~Hz}, 1 \mathrm{H}), 7.45(\mathrm{~d}, J=$ $8.1 \mathrm{~Hz}, 2 \mathrm{H}), 7.18-7.15(\mathrm{~m}, 1 \mathrm{H}), 7.13-7.09(\mathrm{~m}, 2 \mathrm{H}), 6.60(\mathrm{dd}, J=7.4,1.7 \mathrm{~Hz}$, 2H), 5.87 (ddd, $J=16.9,10.3,8.1 \mathrm{~Hz}, 1 \mathrm{H}), 5.12(\mathrm{~d}, J=10.4 \mathrm{~Hz}, 1 \mathrm{H}), 4.85(\mathrm{~d}, J=17.2 \mathrm{~Hz}, 1 \mathrm{H})$, $3.70(\mathrm{dd}, J=12.0,8.1 \mathrm{~Hz}, 1 \mathrm{H}), 3.07(\mathrm{dd}, J=12.1,8.2 \mathrm{~Hz}, 1 \mathrm{H}), 2.56-2.51(\mathrm{~m}, 4 \mathrm{H}), 2.23(\mathrm{~d}, J=$ $13.2 \mathrm{~Hz}, 1 \mathrm{H}) ;{ }^{13} \mathbf{C} \mathbf{N M R}\left(151 \mathrm{MHz}, \mathrm{CDCl}_{3}\right): \delta 169.44,145.87,142.99,141.31,136.46,136.01$, $134.97,130.14(\mathrm{q}, J=33.4 \mathrm{~Hz}), 130.01,129.60,128.68,127.57,127.41,127.35,126.29$ (q, $J=$ $3.8 \mathrm{~Hz}), 126.08$ (q, $J=3.6 \mathrm{~Hz}), 123.59$ (q, $J=272.4 \mathrm{~Hz}), 120.15,50.45,46.89,41.85,21.91$; ${ }^{19}$ F NMR $\left(564 \mathrm{MHz}, \mathrm{CDCl}_{3}\right) \delta$-62.5; ATR-FTIR $\left(\mathbf{c m}^{-1}\right)$ : 3032, 3982, 1717, 1597, 1497, 1416, 1366, 1327, 1265, 1223, 1169, 1126, 1076, 1003, 968, 914, 845, 814, 748, 710, 698, 664, 602, 548; ESI-MS: calculated $\left[\mathrm{C}_{26} \mathrm{H}_{22} \mathrm{~F}_{3} \mathrm{NO}_{3} \mathrm{~S}+\mathrm{Na}\right]^{+}:$508.1165, found: 508.1165; $[\alpha]^{26}{ }_{D}=+41.7\left(\mathrm{c}=1.2, \mathrm{CHCl}_{3}\right)$; The product was analyzed by HPLC to determine the enantiomeric excess: $90 \%$ ee $(\mathrm{AD}-\mathrm{H}$, hexane/i-PrOH $=70 / 30$, detector: $230 \mathrm{~nm}$, flow rate: 1 $\mathrm{mL} / \mathrm{min}), \mathrm{t}_{1}($ minor $)=6.9 \min , \mathrm{t}_{2}($ major $)=12.6 \mathrm{~min}$.

(4S,5R)-7-methyl-4-phenyl-1-tosyl-5-vinyl-1,3,4,5-tetrahydro-2H-benzo[b]azepin-2-one (3ga)

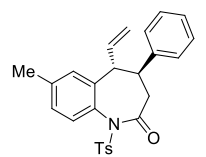

Total yield: $69 \mathrm{mg}(80 \%)$; ${ }^{1} \mathbf{H}$ NMR (300 MHz, $\left.\mathbf{C D C l}_{3}\right) \delta 8.15-8.04(\mathrm{~m}, 2 \mathrm{H})$, $7.44-7.42(\mathrm{~m}, 3 \mathrm{H}), 7.25-7.04(\mathrm{~m}, 5 \mathrm{H}), 6.58-6.53(\mathrm{~m}, 2 \mathrm{H}), 5.96-5.80(\mathrm{~m}, 1 \mathrm{H})$, $5.06(\mathrm{~d}, J=10.5 \mathrm{~Hz}, 1 \mathrm{H}), 4.80(\mathrm{~d}, J=17.1 \mathrm{~Hz}, 1 \mathrm{H}), 3.59(\mathrm{dd}, J=12.0,8.2 \mathrm{~Hz}$, 1H), $3.04(\mathrm{dd}, J=12.0,8.1 \mathrm{~Hz}, 1 \mathrm{H}), 2.63-2.50(\mathrm{~m}, 4 \mathrm{H}), 2.39$ (s, 3H), 2.15 (d, $J=12.4 \mathrm{~Hz}, 1 \mathrm{H})$; ${ }^{13}$ C NMR (75 MHz, $\left.\mathbf{C D C l}_{3}\right) \delta$ 170.22, 145.26, 141.81, 139.40, 138.38, 136.18, 135.73, 133.12, $129.88,129.27,128.87,128.37,128.05,127.32$, 127.28, 126.92, 119.02, 50.66, 46.60, 41.89, 21.77, 21.47. ATR-FTIR ( $\left.\mathbf{c m}^{-1}\right): 3854,3734,3649,2982,1717,1709,1559,1543,1508,1489$, 1366, 1231, 1184, 1169, 1146, 1126, 1088, 876, 814, 745, 725, 698, 667, 625; ESI-MS: calculated $\left[\mathrm{C}_{26} \mathrm{H}_{25} \mathrm{NO}_{3} \mathrm{~S}+\mathrm{Na}\right]^{+}: 454.1447$, found: 454.1439; $[\boldsymbol{\alpha}]^{\mathbf{2 6}}{ }_{\mathbf{D}}=+5.3\left(\mathrm{c}=0.9, \mathrm{CHCl}_{3}\right)$; The product was analyzed by HPLC to determine the enantiomeric excess: $99 \%$ ee (AD-H, hexane/ $i$ PrOH $=70 / 30$, detector: $254 \mathrm{~nm}$, flow rate: $1 \mathrm{~mL} / \mathrm{min}), \mathrm{t}_{1}($ minor $)=13.7 \mathrm{~min}, \mathrm{t}_{2}($ major $)=19.6$ $\min$.

(4S,5R)-7-chloro-4-phenyl-1-tosyl-5-vinyl-1,3,4,5-tetrahydro-2H-benzo[b]azepin-2-one (3ha) Total yield: $62 \mathrm{mg}(68 \%)$; ${ }^{1} \mathbf{H}$ NMR (300 MHz, $\left.\mathbf{C D C l}_{3}\right) \delta 8.11-8.04(\mathrm{~m}, 2 \mathrm{H})$, $7.54-7.33(\mathrm{~m}, 5 \mathrm{H}), 7.19-7.05(\mathrm{~m}, 3 \mathrm{H}), 6.60-6.49$ (m, 2H), 5.82 (ddd, $J=17.2$, $10.4,8.1 \mathrm{~Hz}, 1 \mathrm{H}), 5.10(\mathrm{~d}, J=10.4 \mathrm{~Hz}, 1 \mathrm{H}), 4.83(\mathrm{~d}, J=17.2 \mathrm{~Hz}, 1 \mathrm{H}), 3.58(\mathrm{dd}, J$ 
$=12.0,8.1 \mathrm{~Hz}, 1 \mathrm{H}), 3.06(\mathrm{dd}, J=12.0,8.2 \mathrm{~Hz}, 1 \mathrm{H}), 2.63-2.48(\mathrm{~m}, 4 \mathrm{H}), 2.19(\mathrm{~d}, J=13.0 \mathrm{~Hz}$, 1H); ${ }^{13} \mathbf{C}$ NMR (75 MHz, $\left.\mathbf{C D C l}_{3}\right) \delta 169.75,145.72,141.42,140.69,136.01,135.28,134.94$, 134.32 , 130.51, 129.98, 129.52, 128.60, 127.72, 127.37, 127.25, 127.20, 120.06, 50.43, 46.74, 41.87, 21.91. ATR-FTIR (cm $\left.{ }^{-1}\right): 3028,2982,1709,1593,1489,1455,1420,1231,1169,1142$, 1119, 1088, 930, 880, 814, 748, 725, 698, 667, 625, 540; ESI-MS: calculated $\left[\mathrm{C}_{25} \mathrm{H}_{22} \mathrm{ClNO}_{3} \mathrm{~S}+\right.$ $\mathrm{Na}]^{+}:$474.0901, found: $474.0899 ;[\boldsymbol{\alpha}]^{26}{ }_{\mathbf{D}}=+11.0\left(\mathrm{c}=0.9, \mathrm{CHCl}_{3}\right)$; The product was analyzed by HPLC to determine the enantiomeric excess: $97 \%$ ee $(\mathrm{AD}-\mathrm{H}$, hexane $/ i-\mathrm{PrOH}=70 / 30$, detector: $230 \mathrm{~nm}$, flow rate: $1 \mathrm{~mL} / \mathrm{min}), \mathrm{t}_{1}($ minor $)=13.6 \mathrm{~min}, \mathrm{t}_{2}($ major $)=38.4 \mathrm{~min}$.

(4S,5R)-7-fluoro-4-phenyl-1-tosyl-5-vinyl-1,3,4,5-tetrahydro-2H-benzo[b]azepin-2-one (3ia)

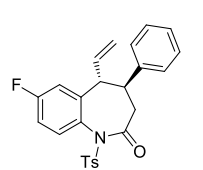
Total yield: $67 \mathrm{mg}$ (77\%); ${ }^{1} \mathbf{H}$ NMR (300 MHz, CDCl $) \delta 8.10-8.06(\mathrm{~m}, 2 \mathrm{H})$, $7.54(\mathrm{dd}, J=8.5,5.2 \mathrm{~Hz}, 2 \mathrm{H}), 7.46-7.42(\mathrm{~m}, 2 \mathrm{H}), 7.18-7.06(\mathrm{~m}, 5 \mathrm{H}), 6.58-$ $6.53(\mathrm{~m}, 2 \mathrm{H}), 5.80(\mathrm{ddd}, J=17.1,10.3,8.1,1 \mathrm{H}), 5.12-5.08(\mathrm{~m}, 1 \mathrm{H}), 4.86-4.80$ (m, 1H), $3.59(\mathrm{dd}, J=12.0,8.1 \mathrm{~Hz}, 1 \mathrm{H}), 3.06(\mathrm{dd}, J=11.7,8.0 \mathrm{~Hz}, 1 \mathrm{H}), 2.60-2.52(\mathrm{~m}, 4 \mathrm{H})$, $2.19(\mathrm{dd}, J=13.0,1.3 \mathrm{~Hz}, 1 \mathrm{H}) ;{ }^{13} \mathbf{C}$ NMR $\left(75 \mathrm{MHz}, \mathrm{CDCl}_{3}\right): \delta 169.82,162.61(\mathrm{~d}, J=249.7 \mathrm{~Hz})$, 145.53, 141.35, 141.23, 135.96, 134.88, 131.58 (d, $J=3.1 \mathrm{~Hz}), 130.92$ (d, $J=9.1 \mathrm{~Hz}), 129.86$, 129.38, 128.47, 127.28, 127.11, 119.87, 114.38 (d, $J=22.9 \mathrm{~Hz}), 113.97$ (d, $J=24.2 \mathrm{~Hz}), 50.29$, 46.68, 41.76, 21.77; ${ }^{19} \mathbf{F}$ NMR $\left(282 \mathrm{MHz}, \mathrm{CDCl}_{3}\right): \delta$ - 110.5; ATR-FTIR (cm $\left.{ }^{-1}\right): 3028,2982$, 1709, 1593, 1489, 1420, 1366, 1231, 1169, 1142, 1119, 1088, 930, 880, 814, 748, 725, 698, 667, 625, 556; ESI-MS: calculated $\left[\mathrm{C}_{25} \mathrm{H}_{22} \mathrm{FNO}_{3} \mathrm{~S}+\mathrm{Na}\right]^{+}:$458.1197, found: 458.1183; $[\alpha]^{26} \mathrm{D}=+3.6\left(\mathrm{c}=1.0, \quad \mathrm{CHCl}_{3}\right)$; The product was analyzed by HPLC to determine the enantiomeric excess: $98 \%$ ee $(\mathrm{AD}-\mathrm{H}$, hexane/i-PrOH $=70 / 30$, detector: $230 \mathrm{~nm}$, flow rate: 1 $\mathrm{mL} / \mathrm{min}), \mathrm{t}_{1}($ minor $)=11.5 \mathrm{~min}, \mathrm{t}_{2}($ major $)=23.3 \mathrm{~min}$.

\section{(4S,5R)-7-bromo-4-phenyl-1-tosyl-5-vinyl-4,5-dihydro-1H-benzo[b]azepin-2(3H)-one (3ja)}

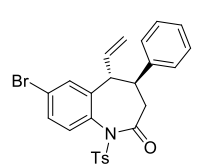

Total yield: $72 \mathrm{mg}$ (73\%); ${ }^{\mathbf{H}} \mathbf{H}$ NMR (400 $\left.\mathbf{M H z}, \mathbf{C D C l}_{3}\right) \delta 7.99(\mathrm{~d}, J=8.4 \mathrm{~Hz}, 2 \mathrm{H})$, $7.52-7.46(\mathrm{~m}, 1 \mathrm{H}), 7.43(\mathrm{t}, J=4.3 \mathrm{~Hz}, 1 \mathrm{H}), 7.36(\mathrm{dd}, J=8.2,5.9 \mathrm{~Hz}, 3 \mathrm{H}), 7.11-$ $6.98(\mathrm{~m}, 3 \mathrm{H}), 6.50-6.43(\mathrm{~m}, 2 \mathrm{H}), 5.74(\mathrm{ddd}, J=17.2,10.3,8.1 \mathrm{~Hz}, 1 \mathrm{H}), 5.03(\mathrm{~d}$, $J=10.5 \mathrm{~Hz}, 1 \mathrm{H}), 4.75(\mathrm{~d}, J=17.2 \mathrm{~Hz}, 1 \mathrm{H}), 3.50(\mathrm{dd}, J=11.9,8.2 \mathrm{~Hz}, 1 \mathrm{H}), 2.99(\mathrm{dd}, J=11.9$, $7.9 \mathrm{~Hz}, 1 \mathrm{H}), 2.53-2.39(\mathrm{~m}, 4 \mathrm{H}), 2.11(\mathrm{dd}, J=13.0,0.9 \mathrm{~Hz}, 1 \mathrm{H}) .{ }^{13} \mathbf{C} \mathbf{N M R}\left(\mathbf{1 0 1} \mathbf{M H z}, \mathbf{C D C l}_{3}\right) \delta$ $169.55,145.60,141.31,140.87,135.91,134.82,134.78,130.66,130.60,130.04,129.86,129.40$, 128.49, 127.26, 127.14, 123.34, 119.96, 50.34, 46.58, 41.75, 21.78. ATR-FTIR (cm-1): 3065, 2924, 1713, 1476, 1365, 1284, 1171, 1126, 1085, 926, 701, 687; ESI-MS: calculated $\left[\mathrm{C}_{25} \mathrm{H}_{22} \mathrm{BrNO}_{3} \mathrm{~S}+\mathrm{Na}^{+}:\right.$518.0396, found: 518.0396; $[\alpha]^{\mathbf{2 6}} \mathrm{D}=+10.9\left(\mathrm{c}=1.9, \mathrm{CHCl}_{3}\right)$; The product 
was analyzed by HPLC to determine the enantiomeric excess: $97 \%$ ee $(\mathrm{AD}-\mathrm{H}$, hexane $/ i-\mathrm{PrOH}=$ 70/30, detector: $254 \mathrm{~nm}$, flow rate: $1 \mathrm{~mL} / \mathrm{min}), \mathrm{t}_{1}($ minor $)=13.6 \mathrm{~min}, \mathrm{t}_{2}($ major $)=39.1 \mathrm{~min}$.

(4S,5R)-7-methoxy-4-phenyl-1-tosyl-5-vinyl-1,3,4,5-tetrahydro-2H-benzo[b]azepin-2-one (3ka)

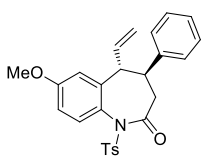

Total yield: $78 \mathrm{mg}(87 \%)$; ${ }^{1} \mathbf{H}$ NMR (300 MHz, $\left.\mathbf{C D C l}_{3}\right) \delta 8.11-8.06(\mathrm{~m}, 2 \mathrm{H})$, $7.53-7.42(\mathrm{~m}, 3 \mathrm{H}), 7.17-7.05(\mathrm{~m}, 3 \mathrm{H}), 6.95-6.88(\mathrm{~m}, 2 \mathrm{H}), 6.55-6.52(\mathrm{~m}$, 2H), $5.83(\mathrm{ddd}, J=17.2,10.4,8.1 \mathrm{~Hz}, 1 \mathrm{H}), 5.06(\mathrm{~d}, J=10.4 \mathrm{~Hz}, 1 \mathrm{H}), 4.80(\mathrm{~d}, J=$ $17.2 \mathrm{~Hz}, 1 \mathrm{H}), 3.87$ (s, 3H), $3.56(\mathrm{dd}, J=12.0,8.1 \mathrm{~Hz}, 1 \mathrm{H}), 3.04$ (dd, $J=11.9,8.2 \mathrm{~Hz}, 1 \mathrm{H}), 2.63-$ $2.56(\mathrm{~m}, 1 \mathrm{H}), 2.52(\mathrm{~s}, 3 \mathrm{H}), 2.15(\mathrm{~d}, J=12.8 \mathrm{~Hz}, 1 \mathrm{H}) ;{ }^{13} \mathbf{C}$ NMR (75 MHz, $\left.\mathbf{C D C l}_{3}\right) \delta$ 170.45, $160.07,145.37,141.86,140.32$, 136.29, 135.59, 130.42, 129.98, 129.40, 128.50, 127.45, 127.07, 119.40, 113.01, 111.88, 55.69, 50.60, 46.81, 42.00, 21.89. ATR-FTIR (cm $\left.{ }^{-1}\right): 3854,3734,3649$, 1717, 1709, 1578, 1559, 1543, 1508, 1489, 1474, 1458, 1362, 1285, 1234, 1169, 1146, 1123, 1084, 1038, 876, 814, 725, 698, 667; ESI-MS: calculated $\left[\mathrm{C}_{26} \mathrm{H}_{25} \mathrm{NO}_{4} \mathrm{~S}+\mathrm{Na}\right]^{+}:$470.1397, found: 470.1396; $[\alpha]^{26} \mathbf{D}=+45.4\left(\mathrm{c}=0.8, \mathrm{CHCl}_{3}\right)$; The product was analyzed by HPLC to determine the enantiomeric excess: $99 \%$ ee $(\mathrm{AD}-\mathrm{H}$, hexane $/ i-\mathrm{PrOH}=70 / 30$, detector: $230 \mathrm{~nm}$, flow rate: 1 $\mathrm{mL} / \mathrm{min}), \mathrm{t}_{1}($ minor $)=16.9$ min, $\mathrm{t}_{2}($ major $)=26.7 \mathrm{~min}$.

(4S,5R)-4-(furan-2-yl)-8-methyl-1-tosyl-5-vinyl-4,5-dihydro-1H-benzo[b]azepin-2(3H)-one (3bk)

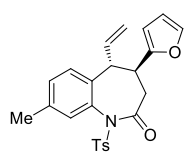

Total yield: 76 mg (90\%); ${ }^{1} \mathbf{H}$ NMR (400 MHz, CDCl $) \delta 7.94(\mathrm{~d}, J=8.4 \mathrm{~Hz}, 2 \mathrm{H})$, $7.33-7.28(\mathrm{~m}, 3 \mathrm{H}), 7.17-7.10(\mathrm{~m}, 2 \mathrm{H}), 7.01(\mathrm{dd}, J=1.8,0.7 \mathrm{~Hz}, 1 \mathrm{H}), 6.06(\mathrm{dd}, J$ $=3.2,1.9 \mathrm{~Hz}, 1 \mathrm{H}), 5.95-5.83(\mathrm{~m}, 1 \mathrm{H}), 5.44(\mathrm{~d}, J=3.3 \mathrm{~Hz}, 1 \mathrm{H}), 5.10(\mathrm{~d}, J=10.4$ $\mathrm{Hz}, 1 \mathrm{H}), 4.85(\mathrm{dt}, J=17.2,1.1 \mathrm{~Hz}, 1 \mathrm{H}), 3.43(\mathrm{dd}, J=11.9,8.0 \mathrm{~Hz}, 1 \mathrm{H}), 3.11(\mathrm{dd}, J=11.9,6.5$ $\mathrm{Hz}, 1 \mathrm{H}), 2.42(\mathrm{~s}, 3 \mathrm{H}), 2.40-2.33(\mathrm{~m}, 4 \mathrm{H}), 2.22(\mathrm{dd}, J=12.8,1.4 \mathrm{~Hz}, 1 \mathrm{H}) .{ }^{13} \mathbf{C}$ NMR $(\mathbf{1 0 1} \mathbf{~ M H z}$, $\left.\mathbf{C D C l}_{3}\right) \delta 169.41,154.81,145.07,141.05,137.63,136.34,135.74,135.67,134.75,130.06$, $129.78,129.66,129.19,126.41,118.64,110.11,105.73,45.13,43.55,40.24,21.69,20.99$. ATRFTIR $\left(\mathbf{c m}^{-1}\right): 2923,1712,1597,1502,1362,1171,1159,1126,1087,1015,912,814,730,667$; ESI-MS: calculated $\left[\mathrm{C}_{24} \mathrm{H}_{23} \mathrm{NO}_{4} \mathrm{~S}+\mathrm{Na}\right]^{+}: 444.1240$, found: $444.1247 ;[\boldsymbol{\alpha}]^{\mathbf{2 6}} \mathbf{D}=+22.8(\mathrm{c}=2.2$, $\mathrm{CHCl}_{3}$ ); The product was analyzed by HPLC to determine the enantiomeric excess: $99 \%$ ee (AS$\mathrm{H}$, hexane $/ i-\mathrm{PrOH}=70 / 30$, detector: $230 \mathrm{~nm}$, flow rate: $1 \mathrm{~mL} / \mathrm{min}), \mathrm{t}_{1}$ (minor) $=9.4 \mathrm{~min}, \mathrm{t}_{2}$ (major) $=14.9 \mathrm{~min}$.

(4S,5R)-4-(furan-2-yl)-7-methyl-1-tosyl-5-vinyl-4,5-dihydro-1H-benzo[b]azepin-2(3H)-one (3gk) 


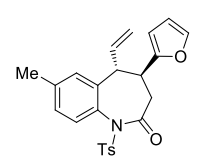

Total yield: $77 \mathrm{mg}$ (92\%); ${ }^{\mathbf{1}} \mathbf{H}$ NMR (400 MHz, CDCl $) \delta 7.94(\mathrm{~d}, J=8.4 \mathrm{~Hz}, 2 \mathrm{H})$, $7.32(\mathrm{dd}, J=15.4,8.0 \mathrm{~Hz}, 3 \mathrm{H}), 7.13(\mathrm{dt}, J=6.2,3.0 \mathrm{~Hz}, 1 \mathrm{H}), 7.09-6.99(\mathrm{~m}, 2 \mathrm{H})$, $6.07(\mathrm{dd}, J=3.2,1.9 \mathrm{~Hz}, 1 \mathrm{H}), 5.89$ (ddd, $J=17.3,10.4,8.0 \mathrm{~Hz}, 1 \mathrm{H}), 5.47$ (d, $J=$ $3.2 \mathrm{~Hz}, 1 \mathrm{H}), 5.11(\mathrm{~d}, J=10.4 \mathrm{~Hz}, 1 \mathrm{H}), 4.85(\mathrm{~d}, J=17.2 \mathrm{~Hz}, 1 \mathrm{H}), 3.44$ (dd, $J=11.8,8.0 \mathrm{~Hz}, 1 \mathrm{H})$, $3.12(\mathrm{dd}, J=11.9,6.6 \mathrm{~Hz}, 1 \mathrm{H}), 2.44-2.39(\mathrm{~m}, 4 \mathrm{H}), 2.33(\mathrm{~s}, 3 \mathrm{H}), 2.26-2.18(\mathrm{~m}, 1 \mathrm{H}) .{ }^{13} \mathbf{C ~ N M R}$ (101 MHz, CDCl $\mathbf{3}) \delta 169.47,154.80,145.05,141.07,139.45,137.54,136.36,135.69,133.28$, $129.62,129.20,128.92,128.24,127.34,118.76,110.14,105.78,45.38,43.46,40.26,21.69,21.43$. ATR-FTIR (cm-1): 2924, 1713, 1597, 1493, 1227, 1170, 1125, 1089, 1015, 913, 814, 733, 674; ESI-MS: calculated $\left[\mathrm{C}_{24} \mathrm{H}_{23} \mathrm{NO}_{4} \mathrm{~S}+\mathrm{Na}\right]^{+}: 444.1240$, found: $444.1247 ;[\boldsymbol{\alpha}]^{\mathbf{2 6}}{ }_{\mathbf{D}}=+26.7(\mathrm{c}=1.9$, $\mathrm{CHCl}_{3}$ ); The product was analyzed by HPLC to determine the enantiomeric excess: $99 \%$ ee (AD$\mathrm{H}$, hexane $/ \mathrm{i}-\mathrm{PrOH}=70 / 30$, detector: $230 \mathrm{~nm}$, flow rate: $1 \mathrm{~mL} / \mathrm{min}), \mathrm{t}_{1}($ major $)=12.5 \mathrm{~min}$, $\mathrm{t}_{2}($ minor $)=13.8 \mathrm{~min}$.

\section{(4S,5R)-7-fluoro-4-(furan-2-yl)-1-tosyl-5-vinyl-4,5-dihydro-1H-benzo[b]azepin-2(3H)-one} (3ik)

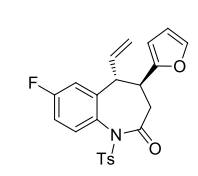

Total yield: $82 \mathrm{mg}(80 \%) ;{ }^{1} \mathbf{H}$ NMR (400 $\left.\mathbf{M H z}, \mathbf{C D C l}_{3}\right) \delta 7.97-7.92(\mathrm{~m}, 2 \mathrm{H}), 7.45$ $(\mathrm{dd}, J=8.8,5.2 \mathrm{~Hz}, 1 \mathrm{H}), 7.32(\mathrm{~d}, J=8.0 \mathrm{~Hz}, 2 \mathrm{H}), 7.07-7.01(\mathrm{~m}, 2 \mathrm{H}), 6.98(\mathrm{dd}, J$ $=9.3,2.9 \mathrm{~Hz}, 1 \mathrm{H}), 6.08(\mathrm{dd}, J=3.2,1.9 \mathrm{~Hz}, 1 \mathrm{H}), 5.82(\mathrm{ddd}, J=17.3,10.4,7.9 \mathrm{~Hz}$, $1 \mathrm{H}), 5.50(\mathrm{~d}, J=3.3 \mathrm{~Hz}, 1 \mathrm{H}), 5.16(\mathrm{~d}, J=10.4 \mathrm{~Hz}, 1 \mathrm{H}), 4.88(\mathrm{dt}, J=17.2,1.0 \mathrm{~Hz}, 1 \mathrm{H}), 3.45(\mathrm{dd}$, $J=11.8,7.9 \mathrm{~Hz}, 1 \mathrm{H}), 3.19-3.11(\mathrm{~m}, 1 \mathrm{H}), 2.43-2.38(\mathrm{~m}, 4 \mathrm{H}), 2.27(\mathrm{dt}, J=7.9,4.3 \mathrm{~Hz}, 1 \mathrm{H}) .{ }^{13} \mathbf{C}$ NMR (101 MHz, CDCl $) \delta 169.06,162.61$ (d, $J=250.0$ Hz), 154.29, 145.31, 141.27, 140.41 (d, $J=7.7 \mathrm{~Hz}), 136.10,134.84,131.74(\mathrm{~d}, J=3.3 \mathrm{~Hz}), 130.98(\mathrm{~d}, J=9.0 \mathrm{~Hz}), 129.61,129.30$, 119.61, $114.56(\mathrm{~d}, J=22.9 \mathrm{~Hz}), 114.05$ (d, $J=24.3 \mathrm{~Hz}), 110.17,109.99,105.99,45.42,43.16$, 40.15, 21.71. ATR-FTIR (cm $\left.{ }^{-1}\right):$ 2924, 1716, 1594, 1364, 1160, 1119, 1086, 912, 814, 730, 670; ESI-MS: calculated $\left[\mathrm{C}_{23} \mathrm{H}_{20} \mathrm{FNO}_{4} \mathrm{~S}+\mathrm{Na}\right]^{+}: 448.0989$, found: $448.0984 ;[\boldsymbol{\alpha}]^{\mathbf{2 6}}{ }_{\mathbf{D}}=+26.7(\mathrm{c}=2.2$, $\mathrm{CHCl}_{3}$ ); The product was analyzed by HPLC to determine the enantiomeric excess: $98 \%$ ee (AD$\mathrm{H}$, hexane $/ i-\mathrm{PrOH}=70 / 30$, detector: $230 \mathrm{~nm}$, flow rate: $1 \mathrm{~mL} / \mathrm{min}), \mathrm{t}_{1}($ minor $)=11.9 \mathrm{~min}$, $\mathrm{t}_{2}$ (major) $=12.8 \mathrm{~min}$.

(4S,5R)-4-(furan-2-yl)-7-methoxy-1-tosyl-5-vinyl-4,5-dihydro-1H-benzo[b]azepin-2(3H)-one (3kk)

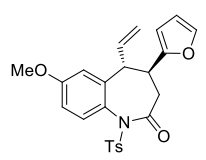

Total yield: $67 \mathrm{mg}(77 \%)$; ${ }^{1} \mathbf{H}$ NMR (400 $\left.\mathbf{M H z}, \mathbf{C D C l}_{3}\right) \delta 7.93(\mathrm{~d}, J=8.4 \mathrm{~Hz}, 2 \mathrm{H})$, $7.39(\mathrm{~d}, J=8.7 \mathrm{~Hz}, 1 \mathrm{H}), 7.30(\mathrm{~d}, J=8.0 \mathrm{~Hz}, 2 \mathrm{H}), 7.02(\mathrm{dd}, J=1.8,0.7 \mathrm{~Hz}, 1 \mathrm{H})$, $6.84(\mathrm{dd}, J=8.7,2.9 \mathrm{~Hz}, 1 \mathrm{H}), 6.78(\mathrm{~d}, J=2.9 \mathrm{~Hz}, 1 \mathrm{H}), 6.07(\mathrm{dd}, J=3.2,1.9 \mathrm{~Hz}$, $1 \mathrm{H}), 5.90-5.79(\mathrm{~m}, 1 \mathrm{H}), 5.46(\mathrm{~d}, J=3.3 \mathrm{~Hz}, 1 \mathrm{H}), 5.12(\mathrm{~d}, J=10.5 \mathrm{~Hz}, 1 \mathrm{H}), 4.90-4.81(\mathrm{~m}, 1 \mathrm{H})$, 
$3.78(\mathrm{~s}, 3 \mathrm{H}), 3.41(\mathrm{dd}, J=11.8,8.0 \mathrm{~Hz}, 1 \mathrm{H}), 3.13(\mathrm{dd}, J=11.9,6.6 \mathrm{~Hz}, 1 \mathrm{H}), 2.45-2.37(\mathrm{~m}, 4 \mathrm{H})$, $2.23(\mathrm{dd}, J=12.8,1.4 \mathrm{~Hz}, 1 \mathrm{H}) .{ }^{13} \mathbf{C}$ NMR (101 MHz, $\left.\mathbf{C D C l}_{3}\right) \delta 169.60,159.97,154.71,145.05$, $141.09,139.33$, 136.31, 135.42, 130.35, 129.59, 129.21, 128.50, 119.00, 112.98, 111.91, 110.14, 105.82, 55.55, 45.46, 43.29, 40.23, 21.69. ATR-FTIR (cm $\left.{ }^{-1}\right):$ 2943, 1712, 1600, 1493, 1363, 1286, 1169, 1123, 1087, 912, 814, 732, 670; ESI-MS: calculated $\left[\mathrm{C}_{24} \mathrm{H}_{23} \mathrm{NO}_{5} \mathrm{~S}+\mathrm{Na}\right]^{+}: 460.1189$, found: $460.1187 ;[\alpha]^{26}{ }_{D}=+24.2\left(\mathrm{c}=2.0, \mathrm{CHCl}_{3}\right)$; The product was analyzed by HPLC to determine the enantiomeric excess: $99 \%$ ee $(\mathrm{AS}-\mathrm{H}$, hexane $/ i-\mathrm{PrOH}=70 / 30$, detector: $230 \mathrm{~nm}$, flow rate: $1 \mathrm{~mL} / \mathrm{min}), \mathrm{t}_{1}($ minor $)=13.7 \mathrm{~min}, \mathrm{t}_{2}($ major $)=32.6 \mathrm{~min}$.

(4S,5R)-4-(furan-2-yl)-6-methoxy-1-tosyl-5-vinyl-4,5-dihydro-1H-benzo[b]azepin-2(3H)-one (3lk)

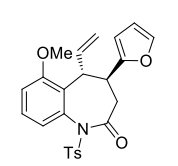

Total yield: $49 \mathrm{mg}(56 \%)$; ${ }^{1} \mathbf{H}$ NMR (400 MHz, $\left.\mathbf{C D C l}_{3}\right) \delta 8.04-8.01(\mathrm{~m}, 2 \mathrm{H}), 7.32-$ $7.22(\mathrm{~m}, 3 \mathrm{H}), 7.07(\mathrm{dd}, J=8.1,0.7 \mathrm{~Hz}, 1 \mathrm{H}), 6.83(\mathrm{dd}, J=8.3,1.9 \mathrm{~Hz}, 2 \mathrm{H}), 6.17-$

$6.13(\mathrm{~m}, 1 \mathrm{H}), 5.88$ (ddd, $J=17.1,10.4,5.8 \mathrm{~Hz}, 1 \mathrm{H}), 5.70(\mathrm{~d}, J=3.2 \mathrm{~Hz}, 1 \mathrm{H}), 5.07$ $(\mathrm{dd}, J=17.1,1.3 \mathrm{~Hz}, 1 \mathrm{H}), 4.90-4.84(\mathrm{~m}, 1 \mathrm{H}), 3.47$ (s, 3H), $3.38(\mathrm{dt}, J=11.6,5.9 \mathrm{~Hz}, 1 \mathrm{H}), 3.33$ - 3.29 (m, 1H), $2.51-2.45(\mathrm{~m}, 1 \mathrm{H}), 2.40$ (s, 3H), $2.37-2.31$ (m, 1H). ${ }^{13} \mathbf{C}$ NMR (101 MHz, $\left.\mathbf{C D C l}_{3}\right) \delta 170.03,157.60,155.87,145.12,141.57,139.27,137.80,136.64,130.13,129.45$, $129.14,127.56,121.07,116.24,111.14,109.94,104.52$, 56.21, 44.38, 42.12, 39.68, 21.72. ATRFTIR $\left(\mathbf{c m}^{-1}\right): 2926,1711,1595,1470,1440,1363,1275,1244,1171,1089,1013,916,814,735$, 662, 600; ESI-MS: calculated $\left[\mathrm{C}_{24} \mathrm{H}_{23} \mathrm{NO}_{5} \mathrm{~S}+\mathrm{Na}\right]^{+}: 460.1189$, found: 460.1181 ; The product was analyzed by HPLC to determine the enantiomeric excess: $93 \%$ ee (AD-H, hexane/ $i-\mathrm{PrOH}=70 / 30$, detector: $230 \mathrm{~nm}$, flow rate: $1 \mathrm{~mL} / \mathrm{min}), \mathrm{t}_{1}($ major $)=9.1 \mathrm{~min}, \mathrm{t}_{2}($ minor $)=10.9 \mathrm{~min}$.

(4S,5R)-4-(4-methoxyphenyl)-8-methyl-1-tosyl-5-vinyl-4,5-dihydro-1H-benzo[b]azepin2(3H)-one (3bc)

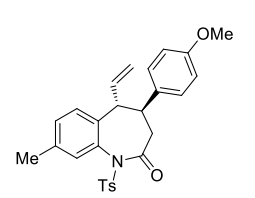

Total yield: $87 \mathrm{mg}(94 \%) ;{ }^{\mathbf{1}} \mathbf{H}$ NMR (400 $\left.\mathbf{M H z}, \mathbf{C D C l}_{3}\right) \delta 8.01(\mathrm{~d}, J=8.3 \mathrm{~Hz}$, $2 \mathrm{H}), 7.36(\mathrm{~d}, J=8.1 \mathrm{~Hz}, 2 \mathrm{H}), 7.28(\mathrm{~s}, 1 \mathrm{H}), 7.21-7.14(\mathrm{~m}, 2 \mathrm{H}), 6.53(\mathrm{~d}, J=8.7$ $\mathrm{Hz}, 2 \mathrm{H}), 6.38(\mathrm{~d}, J=8.7 \mathrm{~Hz}, 2 \mathrm{H}), 5.79$ (ddd, $J=17.4,10.3,8.2 \mathrm{~Hz}, 1 \mathrm{H}), 4.98$ (d, $J=10.5 \mathrm{~Hz}, 1 \mathrm{H}), 4.72(\mathrm{~d}, J=17.2 \mathrm{~Hz}, 1 \mathrm{H}), 3.67$ (s, 3H), $3.50-3.40$ (m, 1H), 2.91 (dd, $J=11.9$, $7.9 \mathrm{~Hz}, 1 \mathrm{H}), 2.52-2.40(\mathrm{~m}, 4 \mathrm{H}), 2.36(\mathrm{~s}, 3 \mathrm{H}), 2.04(\mathrm{~d}, J=12.1 \mathrm{~Hz}, 1 \mathrm{H}) .{ }^{13} \mathbf{C}$ NMR (101 MHz, $\left.\mathbf{C D C l}_{3}\right) \delta 170.27,158.28,145.21,137.34,136.28,135.94,135.75,135.55,133.99,130.01$, 129.93, 129.70, 129.25, 128.28, 126.34, 118.86, 113.70, 55.09, 50.03, 46.57, 42.18, 21.76, 21.00. ATR-FTIR (cm $\left.{ }^{-1}\right):$ 2927, 1700, 1513, 1369, 1251, 1172, 1129, 1087, 911, 829, 731, 657; ESIMS: calculated $\left[\mathrm{C}_{27} \mathrm{H}_{27} \mathrm{NO}_{4} \mathrm{~S}+\mathrm{Na}\right]^{+}: 484.1553$, found: $484.1545 ;[\alpha]^{26}{ }_{\mathrm{D}}=+14.4\left(\mathrm{c}=2.5, \mathrm{CHCl}_{3}\right)$; The product was analyzed by HPLC to determine the enantiomeric excess: $99 \%$ ee (AD-H, 
hexane $/ i-\mathrm{PrOH}=70 / 30$, detector: $254 \mathrm{~nm}$, flow rate: $1 \mathrm{~mL} / \mathrm{min}), \mathrm{t}_{1}($ minor $)=19.3 \mathrm{~min}, \mathrm{t}_{2}$ (major) $=$ $24.3 \mathrm{~min}$.

(4S,5R)-4-(4-methoxyphenyl)-7-methyl-1-tosyl-5-vinyl-4,5-dihydro-1H-benzo[b]azepin2(3H)-one (3gc)

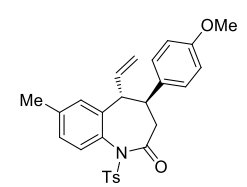

Total yield: $82 \mathrm{mg}$ (89\%); ${ }^{\mathbf{1}} \mathbf{H}$ NMR (400 MHz, $\left.\mathbf{C D C l}_{3}\right) \delta 8.03-7.99(\mathrm{~m}, 2 \mathrm{H})$, $7.38-7.31(\mathrm{~m}, 3 \mathrm{H}), 7.13(\mathrm{dd}, J=8.1,1.4 \mathrm{~Hz}, 1 \mathrm{H}), 7.08(\mathrm{~s}, 1 \mathrm{H}), 6.58-6.50(\mathrm{~m}$, $2 \mathrm{H}), 6.41(\mathrm{~d}, J=8.7 \mathrm{~Hz}, 2 \mathrm{H}), 5.79$ (ddd, $J=17.3,10.3,8.2 \mathrm{~Hz}, 1 \mathrm{H}), 5.04-$ $4.96(\mathrm{~m}, 1 \mathrm{H}), 4.73$ (d, $J=17.2 \mathrm{~Hz}, 1 \mathrm{H}), 3.67$ (s, 3H), 3.47 (dd, $J=11.9,8.2 \mathrm{~Hz}, 1 \mathrm{H}), 2.93$ (dd, $J$ $=11.8,7.9 \mathrm{~Hz}, 1 \mathrm{H}), 2.50-2.39(\mathrm{~m}, 4 \mathrm{H}), 2.34(\mathrm{~s}, 3 \mathrm{H}), 2.04(\mathrm{dd}, J=12.8,0.9 \mathrm{~Hz}, 1 \mathrm{H}) .{ }^{13} \mathbf{C ~ N M R}$ $\left(\mathbf{1 0 1} \mathbf{~ M H z}, \mathbf{C D C l}_{3}\right) \delta 170.33,158.30,145.19,139.36,138.55,136.30,135.90,134.00,133.17$, $129.88,129.26,128.84,128.30,128.01,127.28,118.97,113.73,55.10,49.93,46.80,42.20,21.76$, 21.46. ATR-FTIR $\left(\mathbf{c m}^{-1}\right):$ 2931, 1709, 1513, 1362, 1249, 1170, 1127, 1034, 911, 829, 723, 680; ESI-MS: calculated $\left[\mathrm{C}_{27} \mathrm{H}_{27} \mathrm{NO}_{4} \mathrm{~S}+\mathrm{Na}\right]^{+}: 484.1553$, found: 484.1549; $[\boldsymbol{\alpha}]^{\mathbf{2 6}}{ }_{\mathbf{D}}=+22.1(\mathrm{c}=1.8$, $\mathrm{CHCl}_{3}$ ); The product was analyzed by HPLC to determine the enantiomeric excess: $99 \%$ ee (AD$\mathrm{H}$, hexane $/ \mathrm{i}-\mathrm{PrOH}=70 / 30$, detector: $230 \mathrm{~nm}$, flow rate: $1 \mathrm{~mL} / \mathrm{min}), \mathrm{t}_{1}($ minor $)=22.2 \mathrm{~min}$, $\mathrm{t}_{2}$ (major) $=29.7 \mathrm{~min}$.

(4S,5R)-7-methoxy-4-(4-methoxyphenyl)-1-tosyl-5-vinyl-4,5-dihydro-1H-benzo[b]azepin2(3H)-one (3kc)

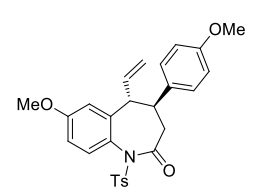

Total yield: $80 \mathrm{mg}(84 \%) ;{ }^{1} \mathbf{H}$ NMR (400 $\left.\mathbf{M H z}, \mathbf{C D C l}_{3}\right) \delta 8.00(\mathrm{~d}, J=8.3 \mathrm{~Hz}$, 2H), $7.42-7.32(\mathrm{~m}, 3 \mathrm{H}), 6.84(\mathrm{ddd}, J=15.2,9.9,2.9 \mathrm{~Hz}, 2 \mathrm{H}), 6.60-6.51(\mathrm{~m}$, 2H), $6.39(\mathrm{~d}, J=8.7 \mathrm{~Hz}, 2 \mathrm{H}), 5.75(\mathrm{ddd}, J=17.3,10.3,8.1 \mathrm{~Hz}, 1 \mathrm{H}), 5.00(\mathrm{~d}, J$ $=10.6 \mathrm{~Hz}, 1 \mathrm{H}), 4.73(\mathrm{~d}, J=17.2 \mathrm{~Hz}, 1 \mathrm{H}), 3.79(\mathrm{~s}, 3 \mathrm{H}), 3.67$ (s, 3H), $3.44(\mathrm{dd}, J=11.9,8.1 \mathrm{~Hz}$, $1 \mathrm{H}), 2.93(\mathrm{dd}, J=11.8,7.9 \mathrm{~Hz}, 1 \mathrm{H}), 2.50(\mathrm{dd}, J=12.7,8.3 \mathrm{~Hz}, 1 \mathrm{H}), 2.45$ (s, 3H), 2.05 (dd, $J=$ 12.7, $0.7 \mathrm{~Hz}, 1 \mathrm{H}) .{ }^{13} \mathbf{C}$ NMR (101 $\left.\mathbf{M H z}, \mathbf{C D C l}_{3}\right) \delta 170.45,159.94,158.32,145.20,140.35$, $136.24,135.63,133.91,130.25,129.85,129.27,128.41,128.30,119.23,113.73,112.87,111.73$, 55.56, 55.10, 49.73, 46.88, 42.17, 21.76. ATR-FTIR (cm $\left.{ }^{-1}\right):$ 2938, 1708, 1609, 1513, 1490, 1362, 1230, 1169, 1087, 1036, 910, 876, 830, 729, 680; ESI-MS: calculated $\left[\mathrm{C}_{27} \mathrm{H}_{27} \mathrm{NO}_{5} \mathrm{~S}+\mathrm{Na}\right]^{+}$: 500.1502, found: $500.1507 ;[\alpha]^{26}{ }_{\mathrm{D}}=+16.8\left(\mathrm{c}=2.4, \mathrm{CHCl}_{3}\right)$; The product was analyzed by HPLC to determine the enantiomeric excess: $99 \%$ ee $(\mathrm{AD}-\mathrm{H}$, hexane $/ i-\mathrm{PrOH}=70 / 30$, detector: $254 \mathrm{~nm}$, flow rate: $1 \mathrm{~mL} / \mathrm{min}), \mathrm{t}_{1}($ minor $)=27.6 \mathrm{~min}, \mathrm{t}_{2}($ major $)=40.7 \mathrm{~min}$. 
(4S,5R)-7-bromo-4-(4-methoxyphenyl)-1-tosyl-5-vinyl-4,5-dihydro-1H-benzo[b]azepin2(3H)-one (3jc)

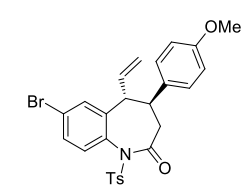

Total yield: $70 \mathrm{mg}(67 \%) ;{ }^{1} \mathbf{H}$ NMR (300 $\left.\mathbf{~ M H z , ~} \mathbf{C D C l}_{3}\right) \delta 7.99(\mathrm{~d}, J=8.3 \mathrm{~Hz}$, 2H), $7.19-7.48(\mathrm{~m}, 5 \mathrm{H}), 6.58-6.52(\mathrm{~m}, 2 \mathrm{H}), 6.40(\mathrm{~d}, J=8.7 \mathrm{~Hz}, 2 \mathrm{H}), 5.73$ (ddd, $J=18.1,10.3,8.2 \mathrm{~Hz}, 1 \mathrm{H}), 5.04(\mathrm{~d}, J=10.4 \mathrm{~Hz}, 1 \mathrm{H}), 4.76(\mathrm{~d}, J=17.2 \mathrm{~Hz}$, $1 \mathrm{H}), 3.67$ (s, 3H), $3.45(\mathrm{dd}, J=11.8,8.2 \mathrm{~Hz}, 1 \mathrm{H}), 2.95(\mathrm{dd}, J=11.9,8.0 \mathrm{~Hz}, 1 \mathrm{H}), 2.50-2.39$ (m, 4H), 2.08 (d, $J=12.8 \mathrm{~Hz}, 1 \mathrm{H}) .{ }^{13} \mathbf{C}$ NMR (75 MHz, $\left.\mathbf{C D C l}_{3}\right) \delta 169.68,158.43,152.73,145.55$, 141.00, 135.94, 134.97, 134.77, 133.43, 130.61, 130.54, 130.37, 130.01, 129.85, 129.39, 128.23, 126.54, 123.31, 119.92, 114.57, 113.81, 55.11, 49.60, 46.78, 42.03, 21.79. ATR-FTIR (cm $\left.{ }^{-1}\right)$ : 2923, 1713, 1671, 1601, 1519, 1365, 1249, 1172, 1125, 1033, 908, 815, 729, 659; ESI-MS: calculated $\left[\mathrm{C}_{26} \mathrm{H}_{24} \mathrm{BrNO}_{4} \mathrm{~S}+\mathrm{Na}\right]^{+}: 550.0482$, found: 550.0482; $[\alpha]^{26} \mathbf{D}=+21.8\left(\mathrm{c}=2.4, \mathrm{CHCl}_{3}\right)$; The product was analyzed by HPLC to determine the enantiomeric excess: $98 \%$ ee (AD-H, hexane $/ i-\mathrm{PrOH}=70 / 30$, detector: $230 \mathrm{~nm}$, flow rate: $1 \mathrm{~mL} / \mathrm{min}), \mathrm{t}_{1}($ minor $)=28.0 \mathrm{~min}, \mathrm{t}_{2}$ (major) $=$ $47.5 \mathrm{~min}$. 


\section{X-ray Crystallography data}

\section{X-Ray diffraction:}

Data sets for the compound 3aa were collected with a D8 Venture Dual Source 100 CMOS diffractometer. Programs used: data collection: APEX2 V2014.5-0 (Bruker AXS Inc., 2014); cell refinement: SAINT V8.34A (Bruker AXS Inc., 2013); data reduction: SAINT V8.34A (Bruker AXS Inc., 2013); absorption correction, SADABS V2014/2 (Bruker AXS Inc., 2014); structure solution SHELXT-2014 (Sheldrick, 2008); structure refinement SHELXL-2014 (Sheldrick, 2008). $R$-values are given for observed reflections, and $w \mathrm{R}^{2}$ values are given for all reflections. Thermals ellipsoids are shown with $50 \%$ probability. $R$-values are given for observed reflections, and $w \mathrm{R}^{2}$ values are given for all reflections.

\section{X-ray crystal structure analysis of 3aa (Figure S3, CCDC Nr.: 1453407):}

A colorless prism-like specimen of $\mathrm{C}_{25} \mathrm{H}_{23} \mathrm{NO}_{3} \mathrm{~S}$, approximate dimensions $0.076 \mathrm{~mm} \times 0.152$ $\mathrm{mm} \times 0.219 \mathrm{~mm}$, was used for the X-ray crystallographic analysis. The X-ray intensity data were measured. A total of 414 frames were collected. The total exposure time was 4.81 hours. The frames were integrated with the Bruker SAINT software package using a wide-frame algorithm. The integration of the data using an orthorhombic unit cell yielded a total of 8035 reflections to a maximum $\theta$ angle of $68.31^{\circ}(0.83 \AA$ resolution), of which 3657 were independent (average redundancy 2.197 , completeness $\left.=98.5 \%, \mathrm{R}_{\text {int }}=3.24 \%, \mathrm{R}_{\text {sig }}=5.27 \%\right)$ and $3439(94.04 \%)$ were

greater than $2 \sigma\left(\mathrm{F}^{2}\right)$. The final cell constants of $\underline{a}=9.7547(3) \AA, \underline{b}=12.6902(3) \AA, \underline{c}=17.1960(4)$ $\AA$, volume $=2128.68(10) \AA^{3}$, are based upon the refinement of the XYZ-centroids of 7422 reflections above $20 \sigma(\mathrm{I})$ with $8.66^{\circ}<2 \theta<136.6^{\circ}$. Data were corrected for absorption effects using the multi-scan method (SADABS). The ratio of minimum to maximum apparent transmission was 0.864 . The calculated minimum and maximum transmission coefficients (based on crystal size) are 0.7260 and 0.8900 . The structure was solved and refined using the Bruker SHELXTL Software Package, using the space group P $2{ }_{1} 2{ }_{1}{ }_{1}$, with $\mathrm{Z}=4$ for the formula unit, $\mathrm{C}_{25} \mathrm{H}_{23} \mathrm{NO}_{3} \mathrm{~S}$. The final anisotropic full-matrix least-squares refinement on $\mathrm{F}^{2}$ with 272 variables converged at $\mathrm{R} 1=3.20 \%$, for the observed data and $\mathrm{wR} 2=7.61 \%$ for all data. The goodness-offit was 1.079. The largest peak in the final difference electron density synthesis was $0.149 \mathrm{e}^{-} / \AA^{3}$ and the largest hole was $-0.324 \mathrm{e}^{-} / \AA^{3}$ with an RMS deviation of $0.045 \mathrm{e}^{-} / \AA^{3}$. On the basis of the final model, the calculated density was $1.303 \mathrm{~g} / \mathrm{cm}^{3}$ and $\mathrm{F}(000), 880 \mathrm{e}^{-}$. 


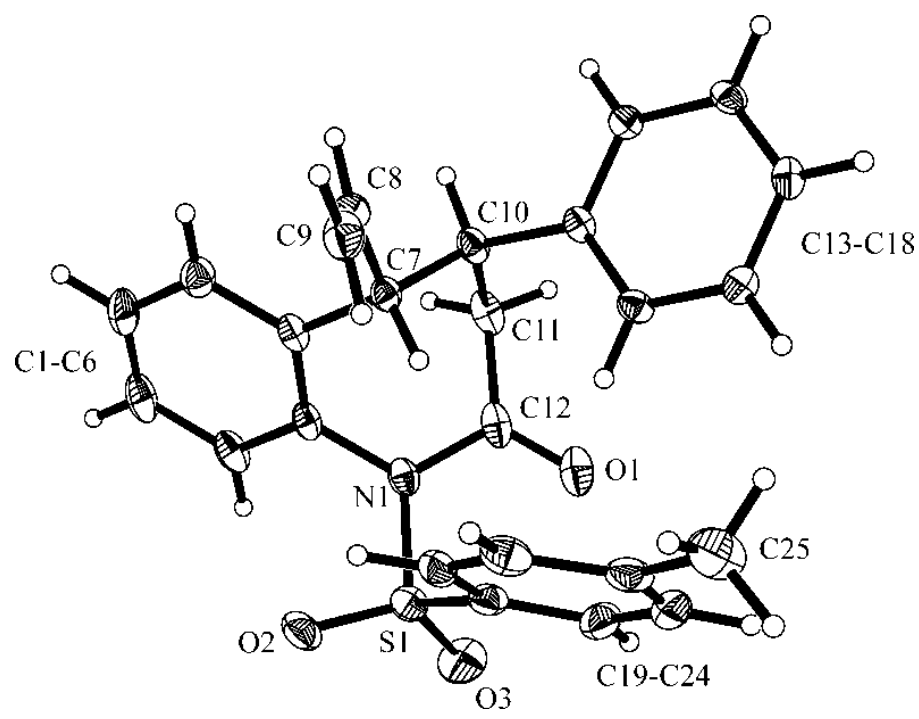

Figure S3| Crystal structure of compound 3aa (CCDC Nr.: 1453407).

(Thermals ellipsoids are shown with $50 \%$ probability.)

\section{Synthetic Transformation of 3aa}

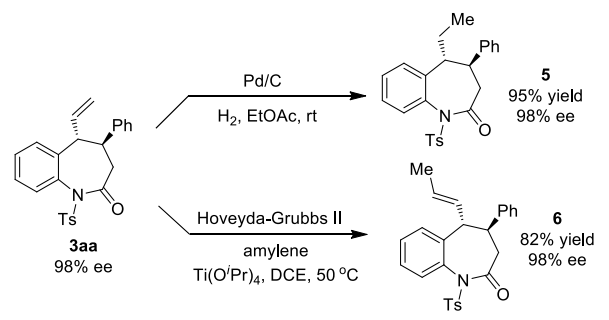

A suspension of $\mathrm{Pd} / \mathrm{C}(10 \mathrm{mg})$ and $3 \mathbf{a a}(41.7 \mathrm{mg}, 0.1 \mathrm{mmol})$ in ethyl acetate $(2.5 \mathrm{~mL})$ was stirred at r.t. under $1 \mathrm{~atm}$ hydrogen atmosphere. After being stirred $3 \mathrm{~h}$, the mixture was filtrated through a pad of Celite and the filtration was concentrated in vacuo, the residue was purified by column chromatography on silica gel to afford the desired the product $\mathbf{5}$ in $95 \%$ yield $(39 \mathrm{mg}, 98 \%$ e.e.).

${ }^{1}$ H NMR (400 MHz, $\left.\mathbf{C D C l}_{3}\right) \delta 8.03(\mathrm{dd}, J=21.8,8.4 \mathrm{~Hz}, 2 \mathrm{H}), 7.51(\mathrm{dd}, J=7.7,1.4 \mathrm{~Hz}, 1 \mathrm{H})$, $7.42-7.29(\mathrm{~m}, 5 \mathrm{H}), 7.10(\mathrm{dd}, J=8.3,6.3 \mathrm{~Hz}, 1 \mathrm{H}), 7.04(\mathrm{t}, J=7.3 \mathrm{~Hz}, 2 \mathrm{H}), 6.48(\mathrm{~d}, J=7.2 \mathrm{~Hz}$, 2H), $2.75-2.61(\mathrm{~m}, 2 \mathrm{H}), 2.46(\mathrm{~s}, 3 \mathrm{H}), 2.38(\mathrm{dd}, J=10.4,5.3 \mathrm{~Hz}, 1 \mathrm{H}), 2.02(\mathrm{~d}, J=12.8 \mathrm{~Hz}, 1 \mathrm{H})$, $1.64-1.56(\mathrm{~m}, 1 \mathrm{H}), 1.28-1.21(\mathrm{~m}, 1 \mathrm{H}), 0.74(\mathrm{t}, J=7.3 \mathrm{~Hz}, 3 \mathrm{H}) .{ }^{13} \mathbf{C}$ NMR (101 MHz, CDCl $)$ $\delta 170.31,145.23,142.53,137.65,137.39,136.13,129.99,129.50,129.25,128.55,127.22,127.16$, 126.98, 126.22, 125.63, 52.24, 44.49, 42.08, 21.84, 21.76, 12.35. ATR-FTIR (cm $\left.{ }^{-1}\right): 3064,2963$, 1709, 1598, 1365, 1170, 1097, 977, 734, 609; ESI-MS: calculated $\left[\mathrm{C}_{25} \mathrm{H}_{25} \mathrm{NO}_{3} \mathrm{~S}+\mathrm{Na}\right]^{+}$: 
442.1447, found: $442.1470 ;[\alpha]^{26}{ }_{\mathrm{D}}=+26.0\left(\mathrm{c}=1.7, \mathrm{CHCl}_{3}\right)$; The product was analyzed by HPLC to determine the enantiomeric excess: $98 \%$ ee $(\mathrm{AD}-\mathrm{H}$, hexane $/ i$-PrOH $=70 / 30$, detector: $254 \mathrm{~nm}$, flow rate: $1 \mathrm{~mL} / \mathrm{min}), \mathrm{t}_{1}($ minor $)=9.9 \mathrm{~min}, \mathrm{t}_{2}($ major $)=12.2 \mathrm{~min}$.

To a solution of 3aa $(41.7 \mathrm{mg}, 0.1 \mathrm{mmol})$, amylene $(1.0 \mathrm{~mL})$ in 1,2-dichloroethane $(3 \mathrm{~mL}), 50$ mol\% of $\operatorname{Ti}\left(\mathrm{O}^{i} \mathrm{Pr}\right)_{4}(14.2 \mathrm{mg}, 0.05 \mathrm{mmol})$ was added. Then the solution was stirred for $1 \mathrm{~h}$. Hoveyda-Grubbs II catalyst $(6.3 \mathrm{mg}, 0.01 \mathrm{mmol})$ was added and reaction mixture was stirring at $50{ }^{\circ} \mathrm{C}$ for $3 \mathrm{~h}$. After removing solvents, the residue was purified by column chromatography to give the product 6 in $82 \%$ yield $\left(35 \mathrm{mg}, 98 \%\right.$ e.e.). ${ }^{2,3}$

${ }^{1}$ H NMR (400 MHz, CDCl $) \delta 8.01(\mathrm{~d}, J=8.3 \mathrm{~Hz}, 2 \mathrm{H}), 7.49-7.44(\mathrm{~m}, 1 \mathrm{H}), 7.35-7.32(\mathrm{~m}, 4 \mathrm{H})$, $7.07-6.96(\mathrm{~m}, 4 \mathrm{H}), 6.48(\mathrm{~d}, J=6.9 \mathrm{~Hz}, 2 \mathrm{H}), 5.46-5.38(\mathrm{~m}, 1 \mathrm{H}), 5.15-5.07(\mathrm{~m}, 1 \mathrm{H}), 3.51$ (dd, $J=11.9,8.4 \mathrm{~Hz}, 1 \mathrm{H}), 2.90(\mathrm{dd}, J=12.0,7.8 \mathrm{~Hz}, 1 \mathrm{H}), 2.46(\mathrm{~s}, 3 \mathrm{H}), 2.36-2.25(\mathrm{~m}, 1 \mathrm{H}), 2.10-$ $2.05(\mathrm{~m}, 1 \mathrm{H}), 1.47(\mathrm{dd}, J=6.4,1.3 \mathrm{~Hz}, 3 \mathrm{H}) .{ }^{13} \mathbf{C}$ NMR (101 MHz, $\left.\mathbf{C D C l}_{3}\right) \delta$ 170.14, 145.23, 142.05, 139.53, 136.26, 135.73, 129.92, 129.64, 129.36, 129.26, 129.14, 129.01, 128.32, 128.28, $127.42,127.38,127.21,126.81,126.77,51.16,45.78,41.90,21.75,18.09$. ATR-FTIR $\left(\mathbf{c m}^{-1}\right)$ : 2923, 1710, 1451, 1363, 1291, 1261, 1170, 1087, 972, 734, 653, 607; ESI-MS: calculated $\left[\mathrm{C}_{26} \mathrm{H}_{25} \mathrm{NO}_{3} \mathrm{~S}+\mathrm{Na}\right]^{+}: 454.1447$, found: 454.1431; $[\boldsymbol{\alpha}]^{26}{ }_{\mathrm{D}}=-18.9\left(\mathrm{c}=1.6, \mathrm{CHCl}_{3}\right)$; The product was analyzed by HPLC to determine the enantiomeric excess: $98 \%$ ee (OD-H, hexane $/ i-\mathrm{PrOH}=$ 90/10, detector: $254 \mathrm{~nm}$, flow rate: $1 \mathrm{~mL} / \mathrm{min}), \mathrm{t}_{1}($ minor $)=12.9 \mathrm{~min}, \mathrm{t}_{2}($ major $)=28.8 \mathrm{~min}$.

\section{References}

1. Wang, C.; Tunge, J. A. J. Am. Chem. Soc. 2008, 130, 8118.

2. Yang, Q.; Xiao, W.-J.; Yu, Z.-K. Org. Lett. 2005, 7, 871.

3. Li, T.-R.; Tan, F.; Lu, L.-Q.; Wei, Y.; Wang, Y.-N.; Liu, Y.-Y.; Yang Q.-Q.; Chen, J.-R.; Shi, D.-Q.; Xiao, W.-J. Nat. Commun. 2014, 5, 5500. 


\section{NMR spectra}

${ }^{1} \mathrm{H}$ NMR spectrum of 3aa
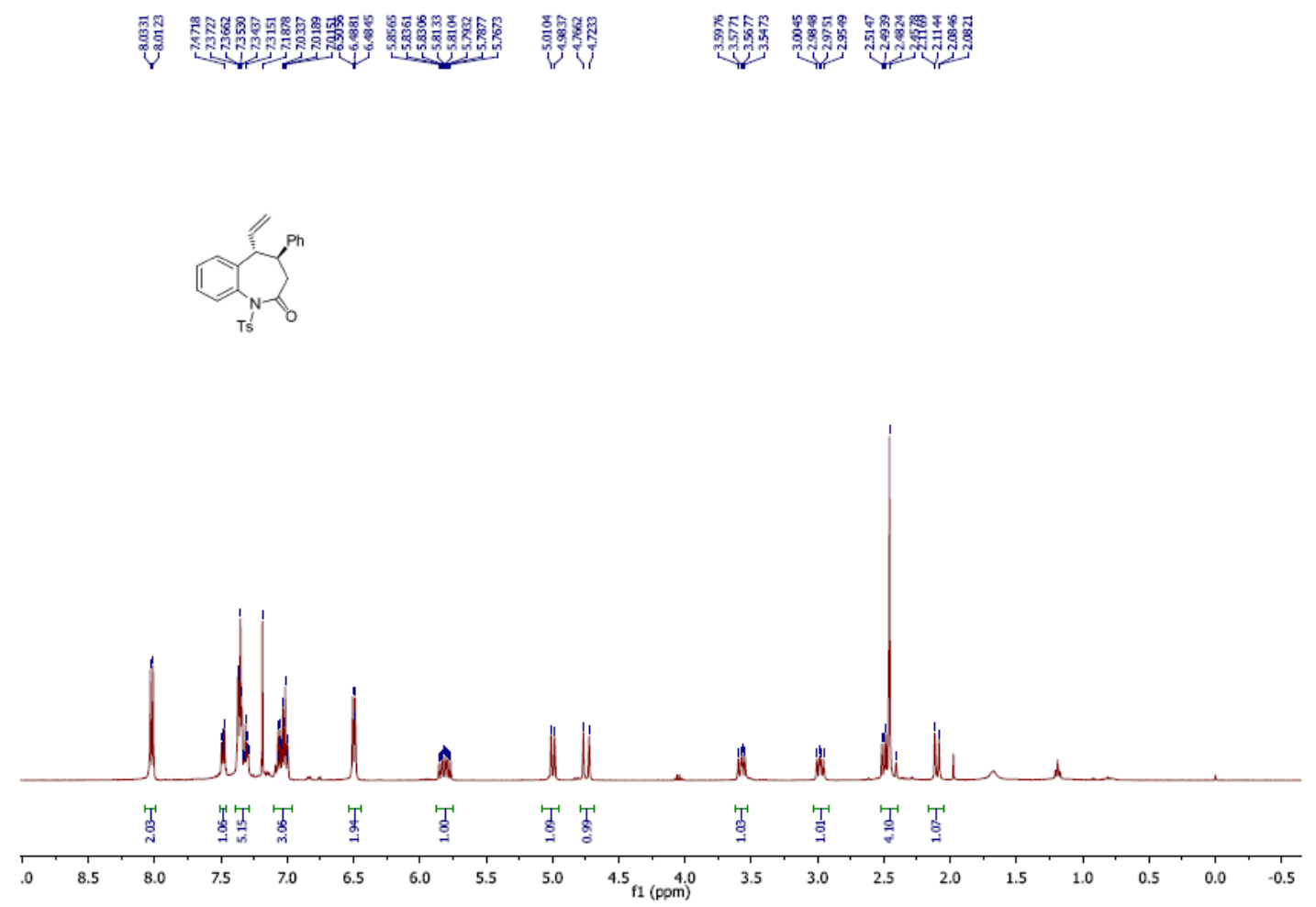

${ }^{13} \mathrm{C}$ NMR spectrum of $\mathbf{3 a a}$

\%
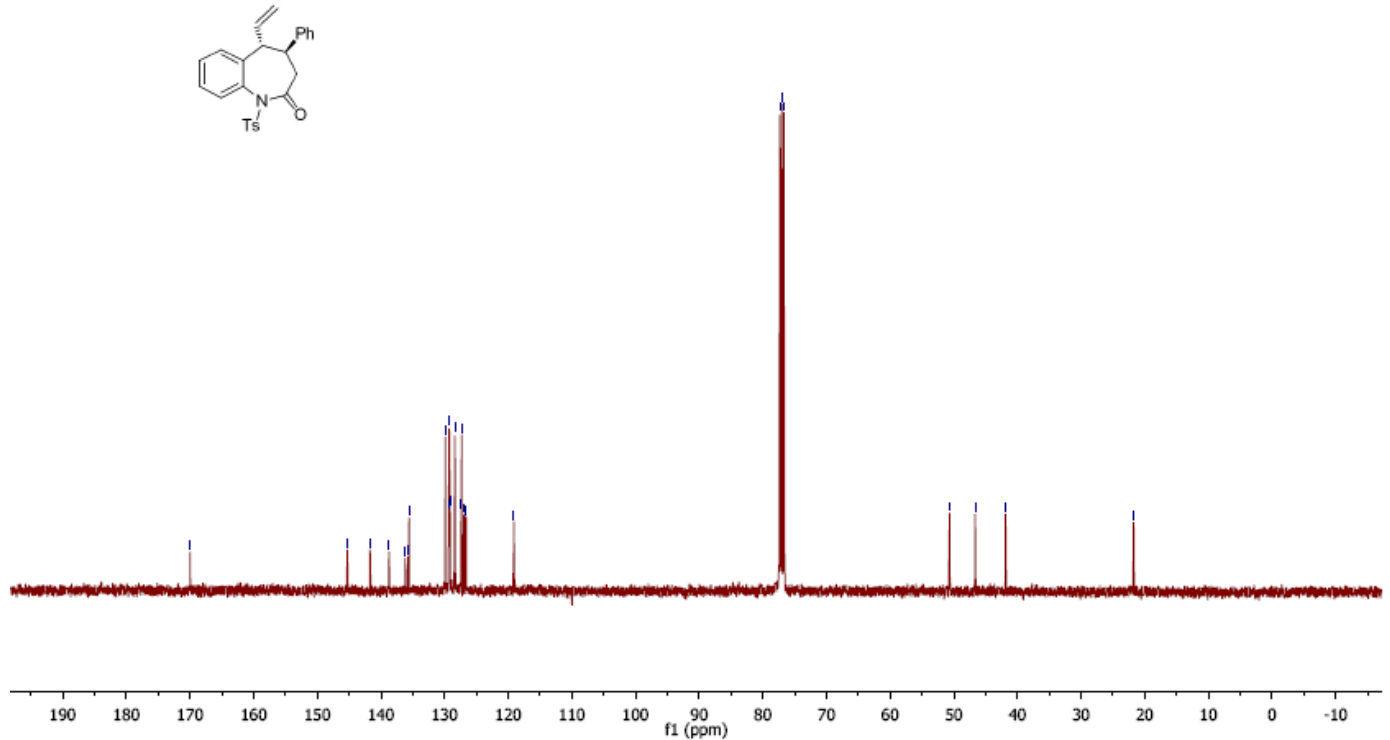
${ }^{1} \mathrm{H}$ NMR spectrum of $\mathbf{3 a b}$

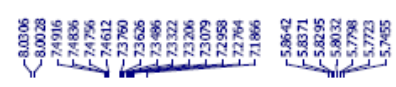

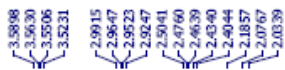
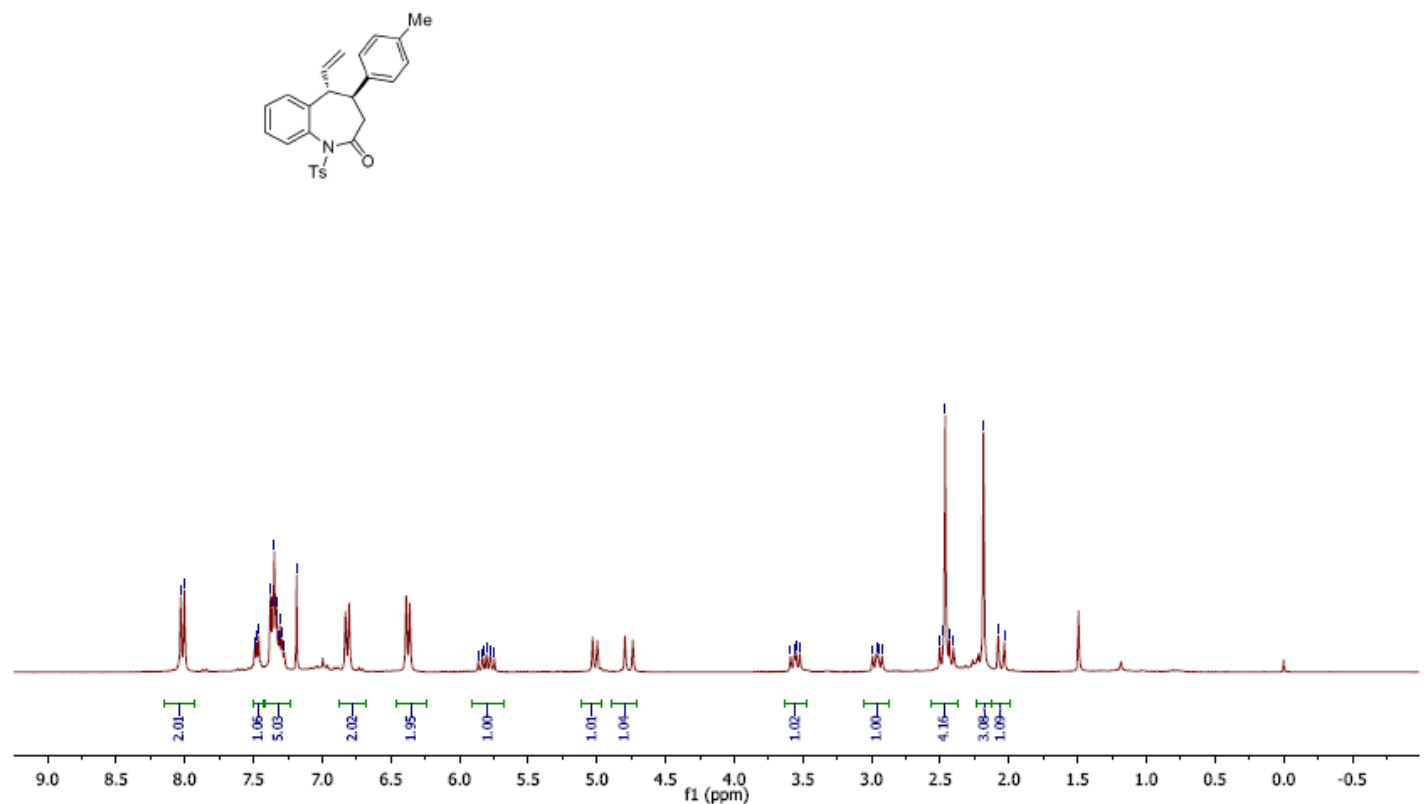

${ }^{13} \mathrm{C}$ NMR spectrum of $\mathbf{3 a b}$

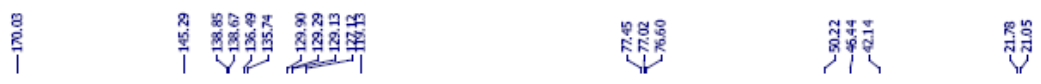
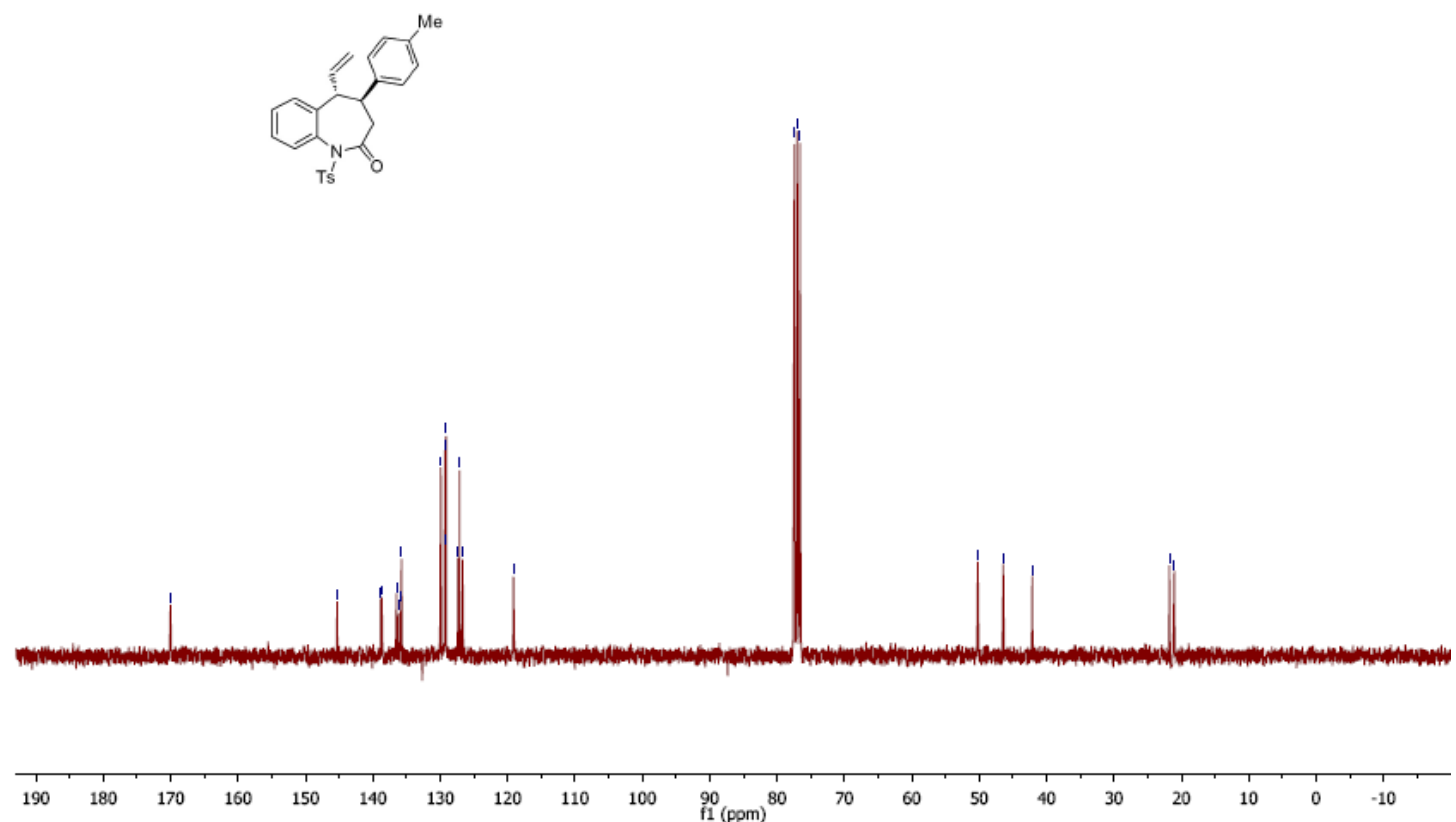
${ }^{1} \mathrm{H}$ NMR spectrum of $\mathbf{3 a c}$
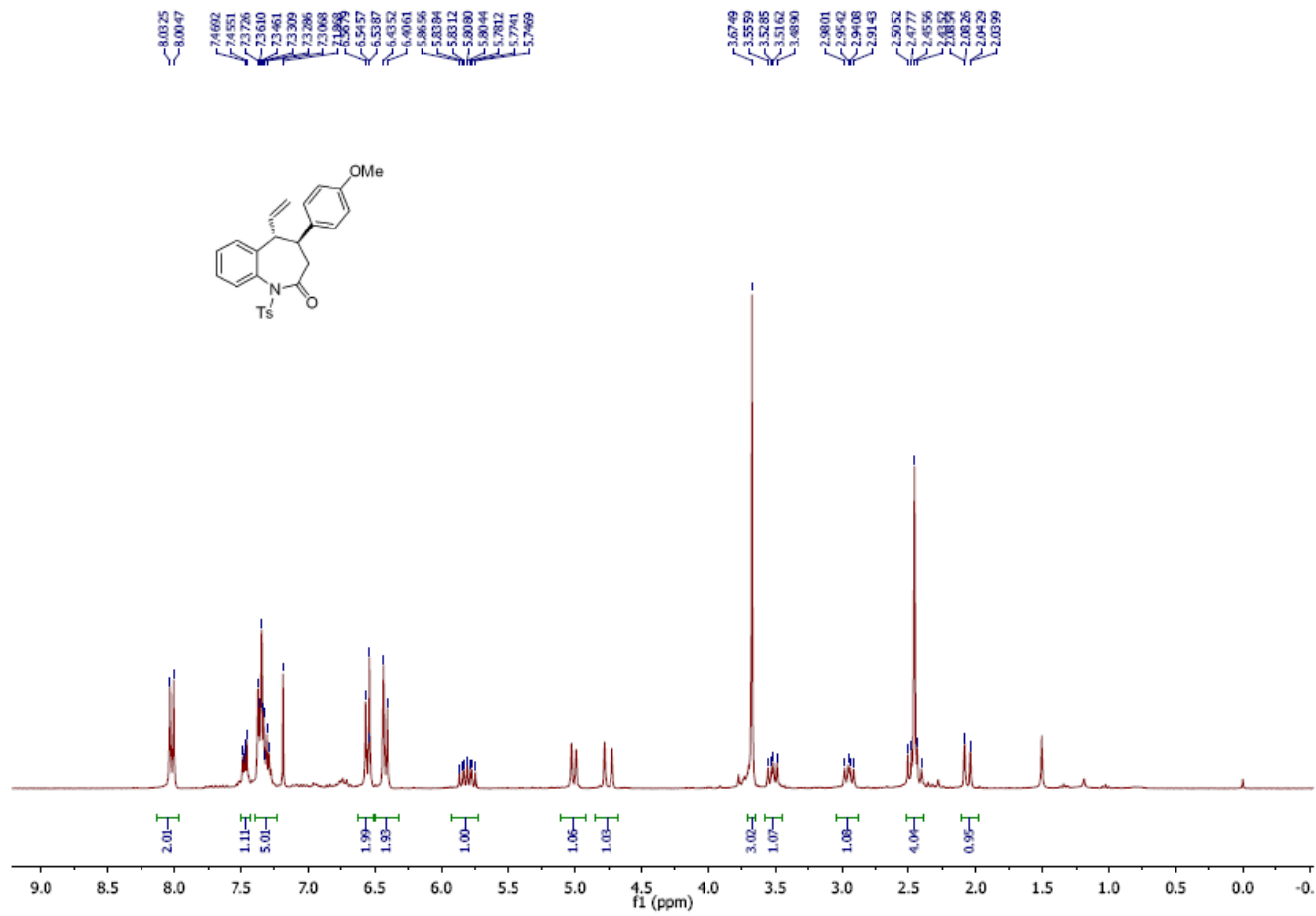

${ }^{13} \mathrm{C}$ NMR spectrum of $\mathbf{3 a c}$
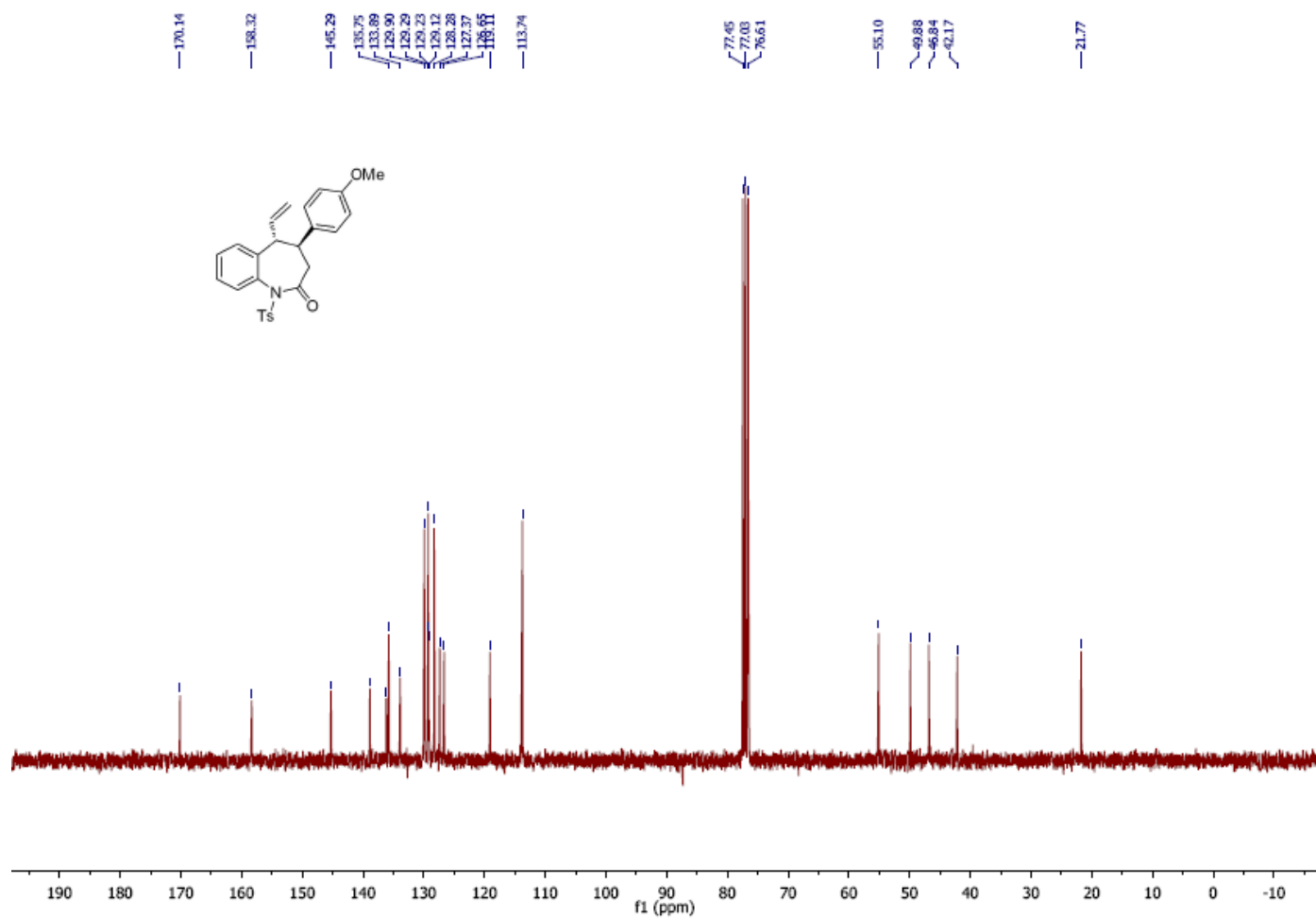
${ }^{1} \mathrm{H}$ NMR spectrum of $\mathbf{3 a d}$
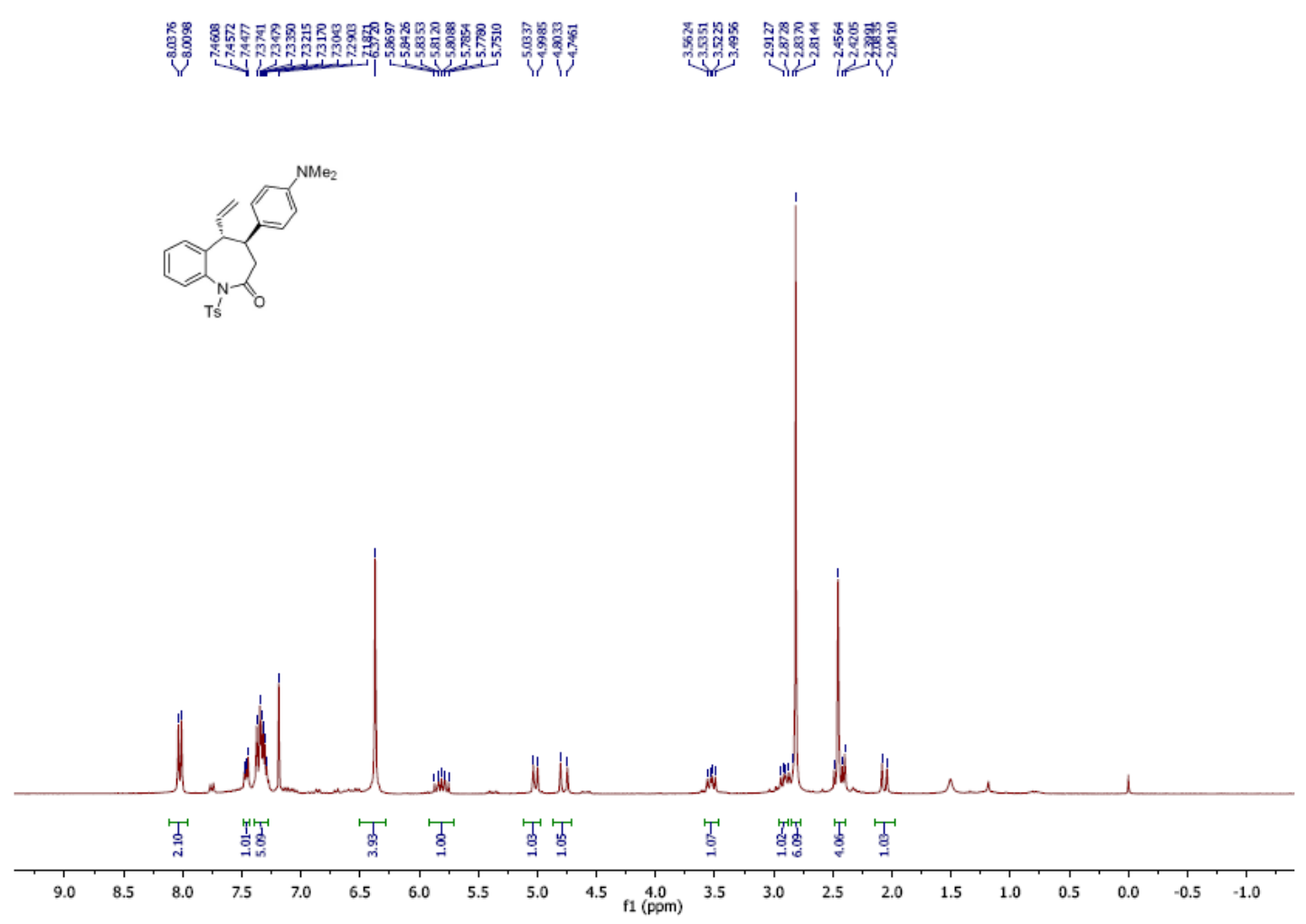

${ }^{13} \mathrm{C}$ NMR spectrum of $\mathbf{3 a d}$
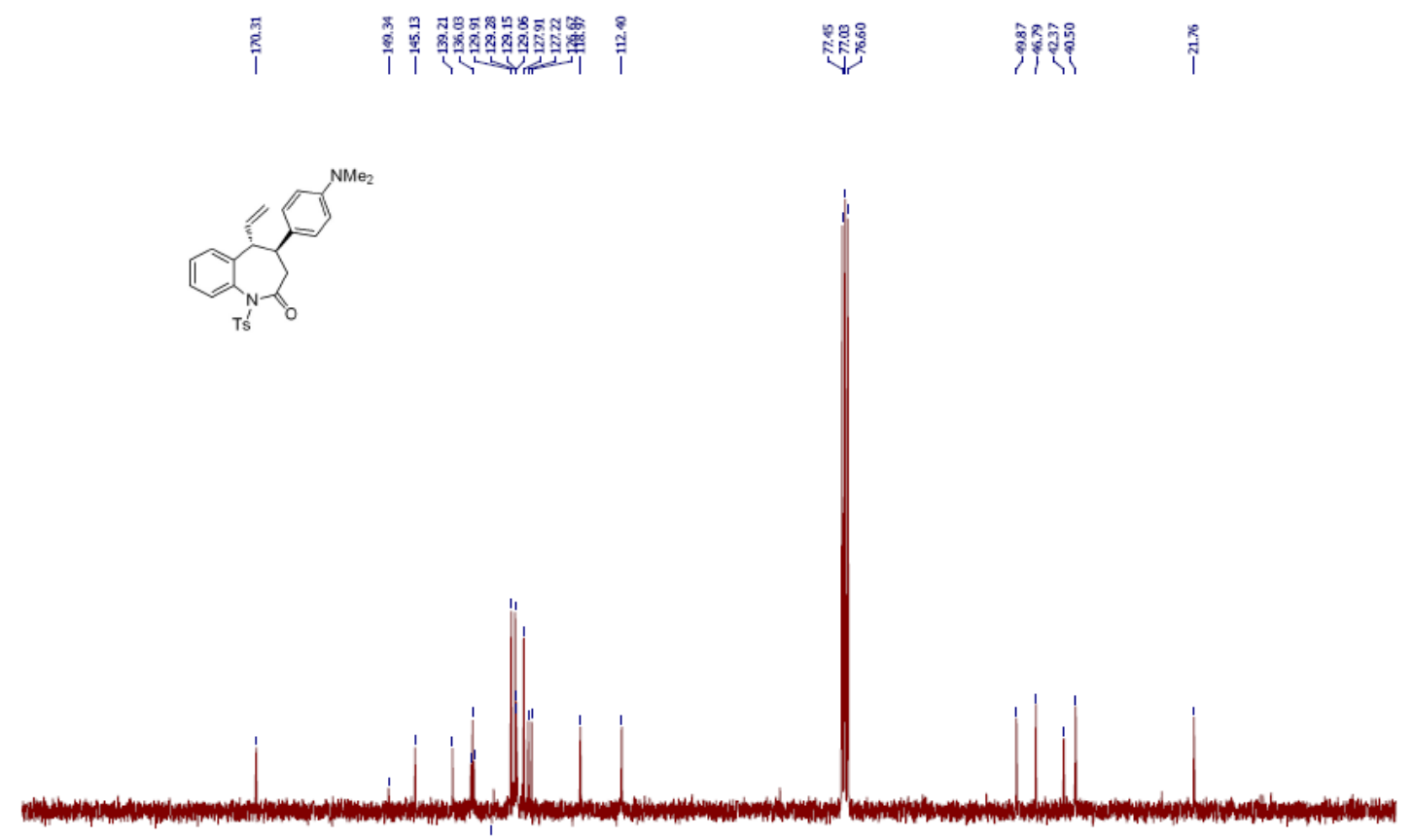
${ }^{1} \mathrm{H}$ NMR spectrum of $\mathbf{3 a e}$
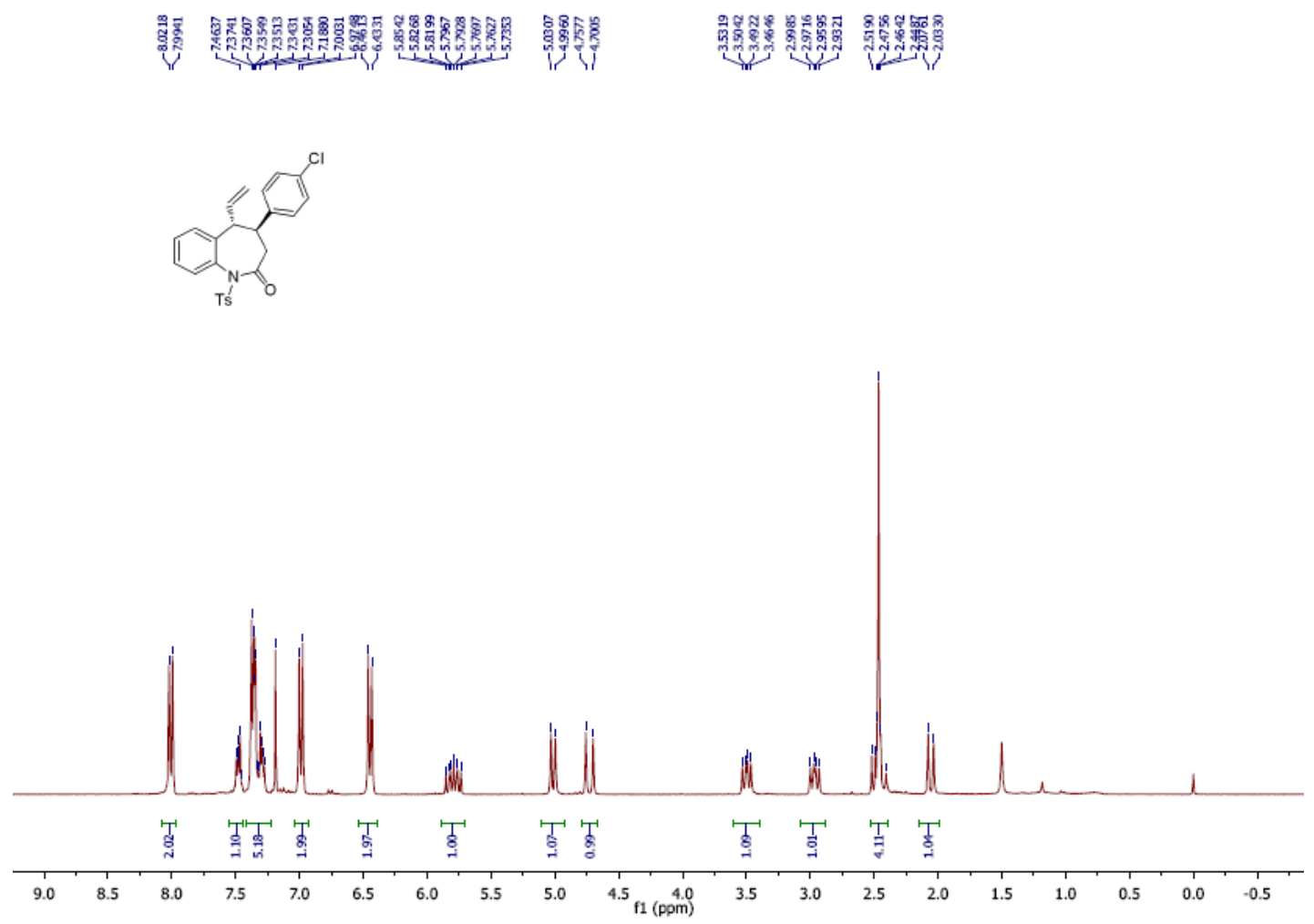

${ }^{13} \mathrm{C}$ NMR spectrum of 3ae
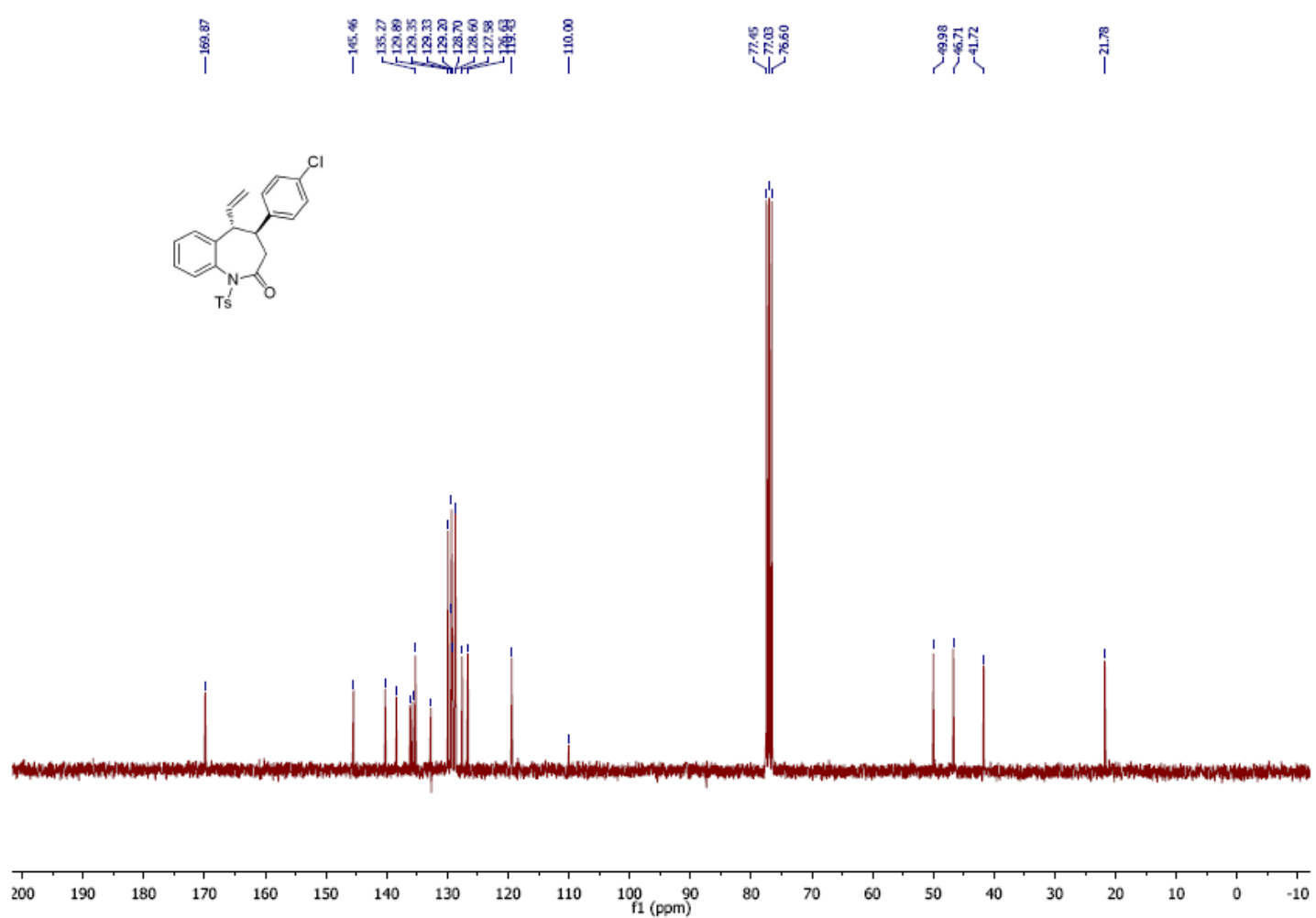
${ }^{1} \mathrm{H}$ NMR spectrum of $\mathbf{3 a f}$
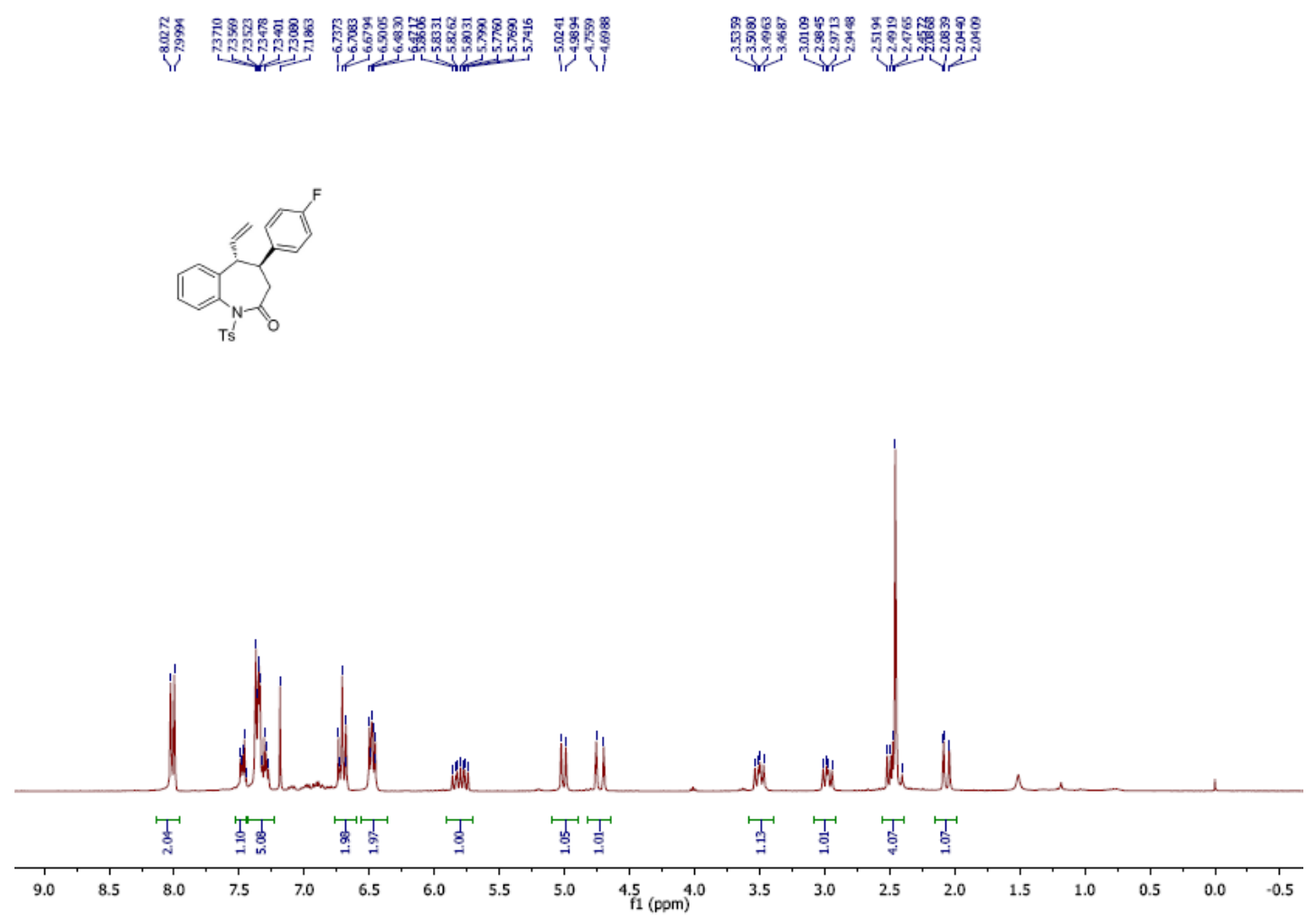

${ }^{13} \mathrm{C}$ NMR spectrum of $3 \mathbf{a f}$
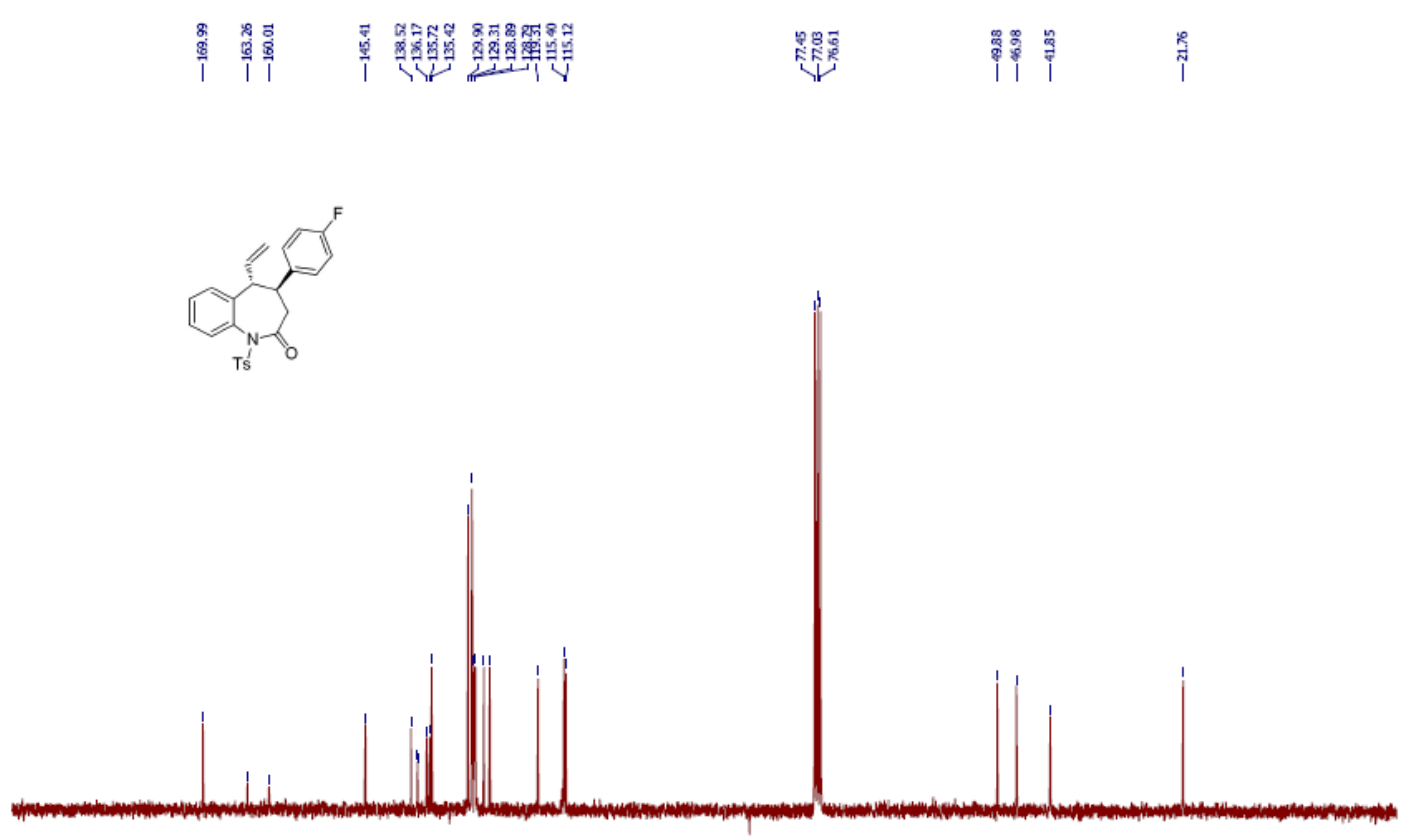
${ }^{1} \mathrm{H}$ NMR spectrum of $\mathbf{3 a g}$
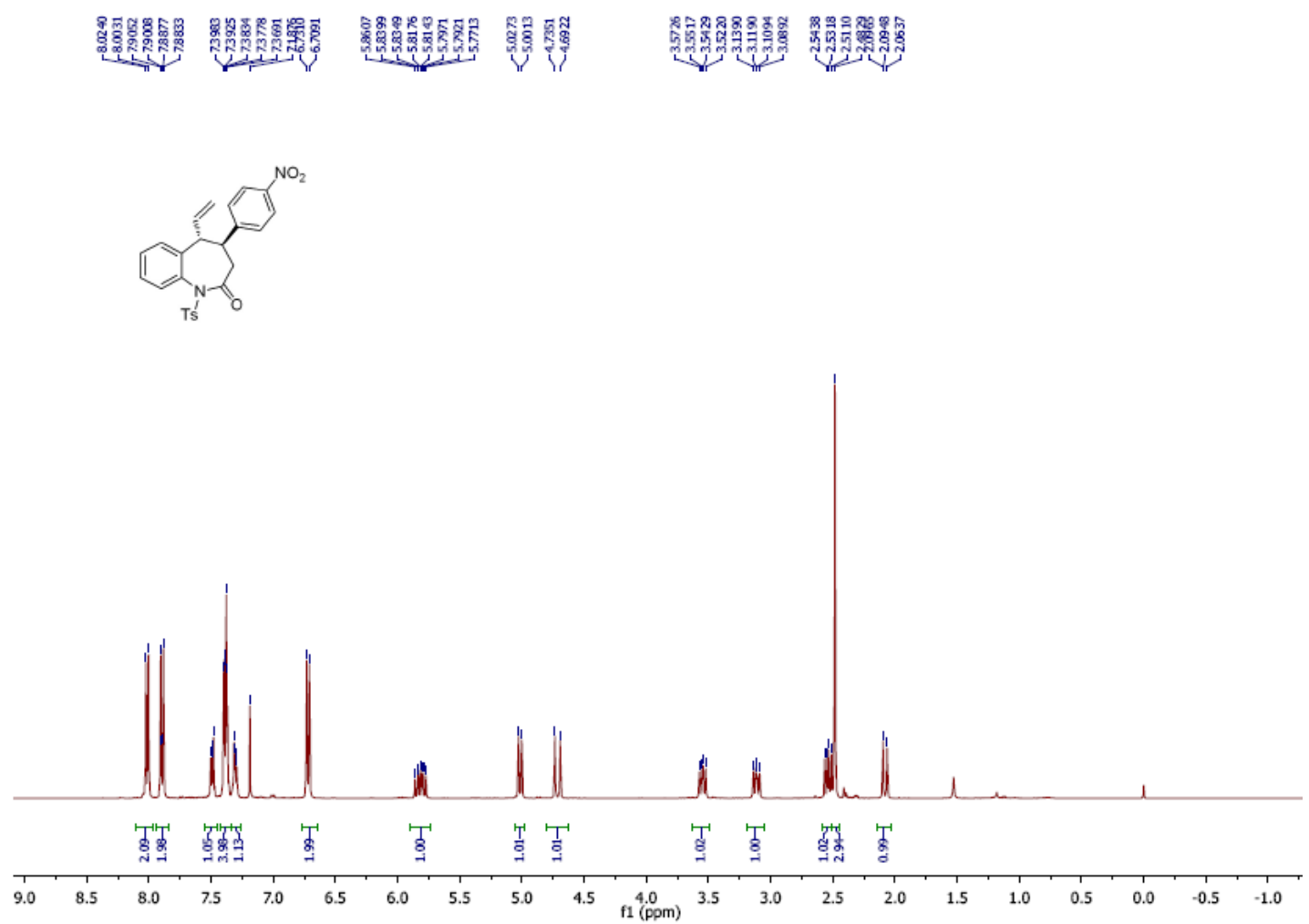

${ }^{13} \mathrm{C}$ NMR spectrum of $\mathbf{3 a g}$

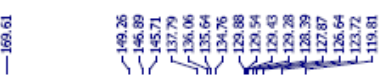

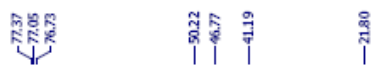
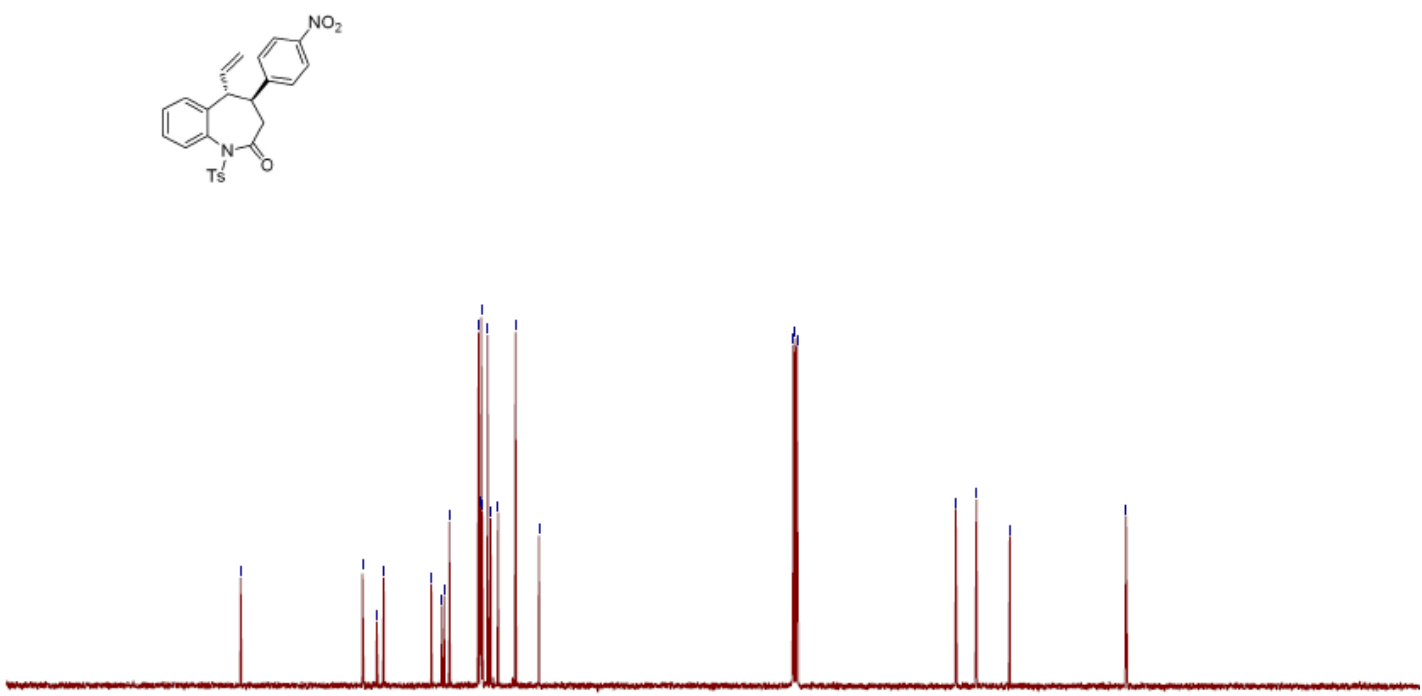

$\begin{array}{lllllllllllllllllllllllllllllllll}200 & 190 & 180 & 170 & 160 & 150 & 140 & 130 & 120 & 110 & 100 & 90 & 90 & 70 & 60 & 50 & 40 & 30 & 20 & 10 & 0 & -10 & -20\end{array}$ 
${ }^{1} \mathrm{H}$ NMR spectrum of $\mathbf{3 a h}$
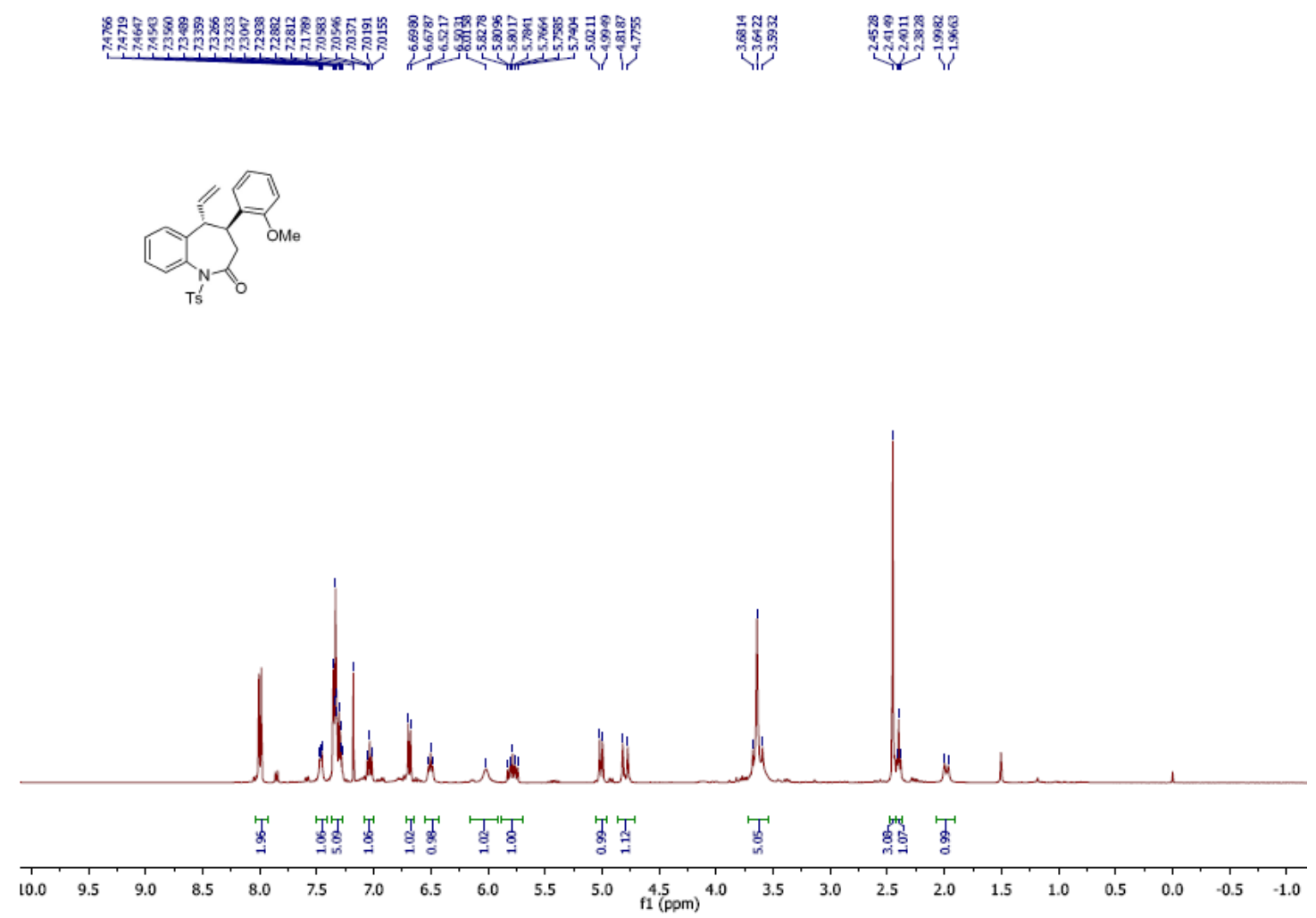

${ }^{13} \mathrm{C}$ NMR spectrum of $\mathbf{3 a h}$
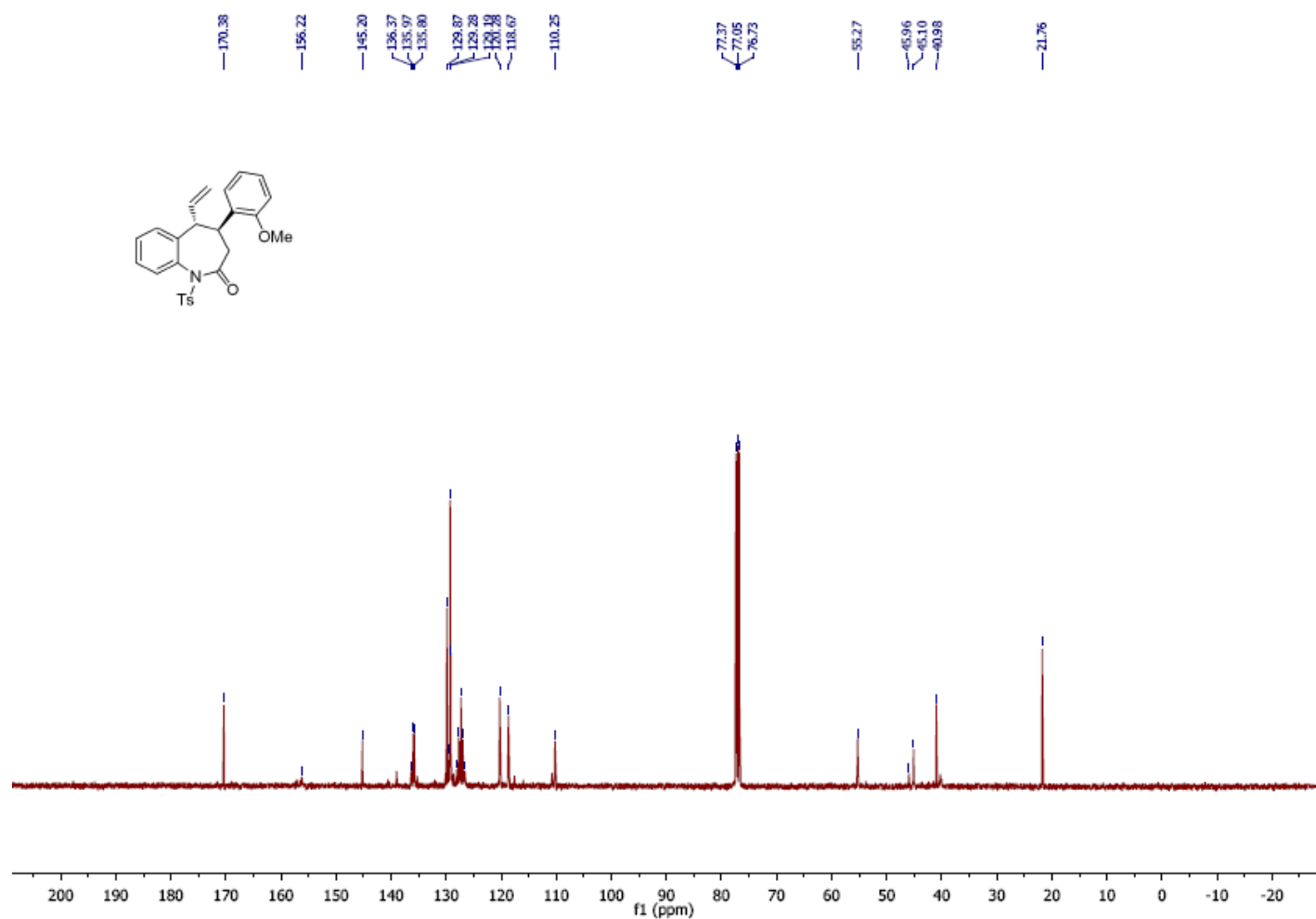
${ }^{1} \mathrm{H}$ NMR spectrum of 3ai
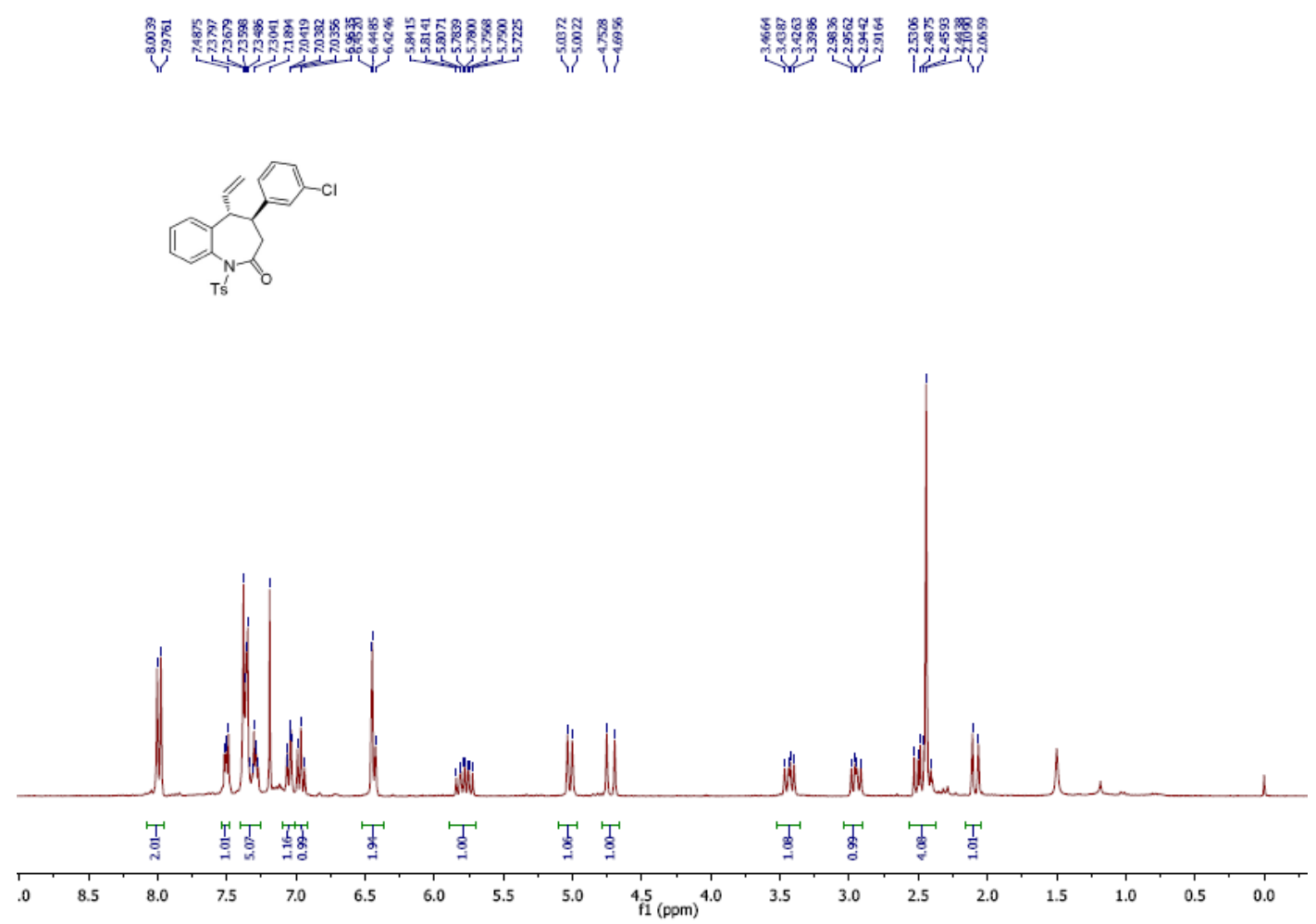

${ }^{13} \mathrm{C}$ NMR spectrum of 3 ai
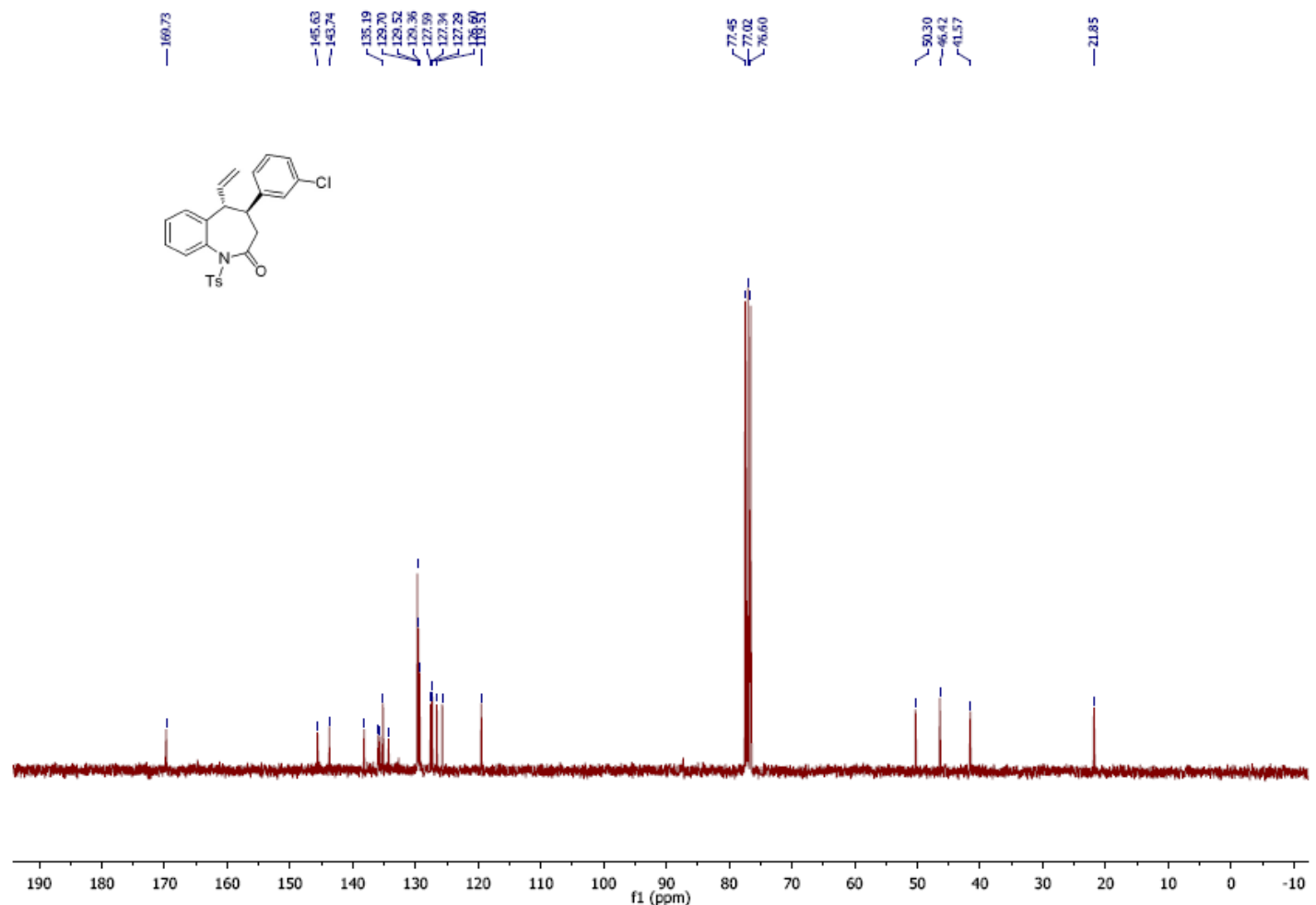
${ }^{1} \mathrm{H}$ NMR spectrum of $\mathbf{3 a j}$
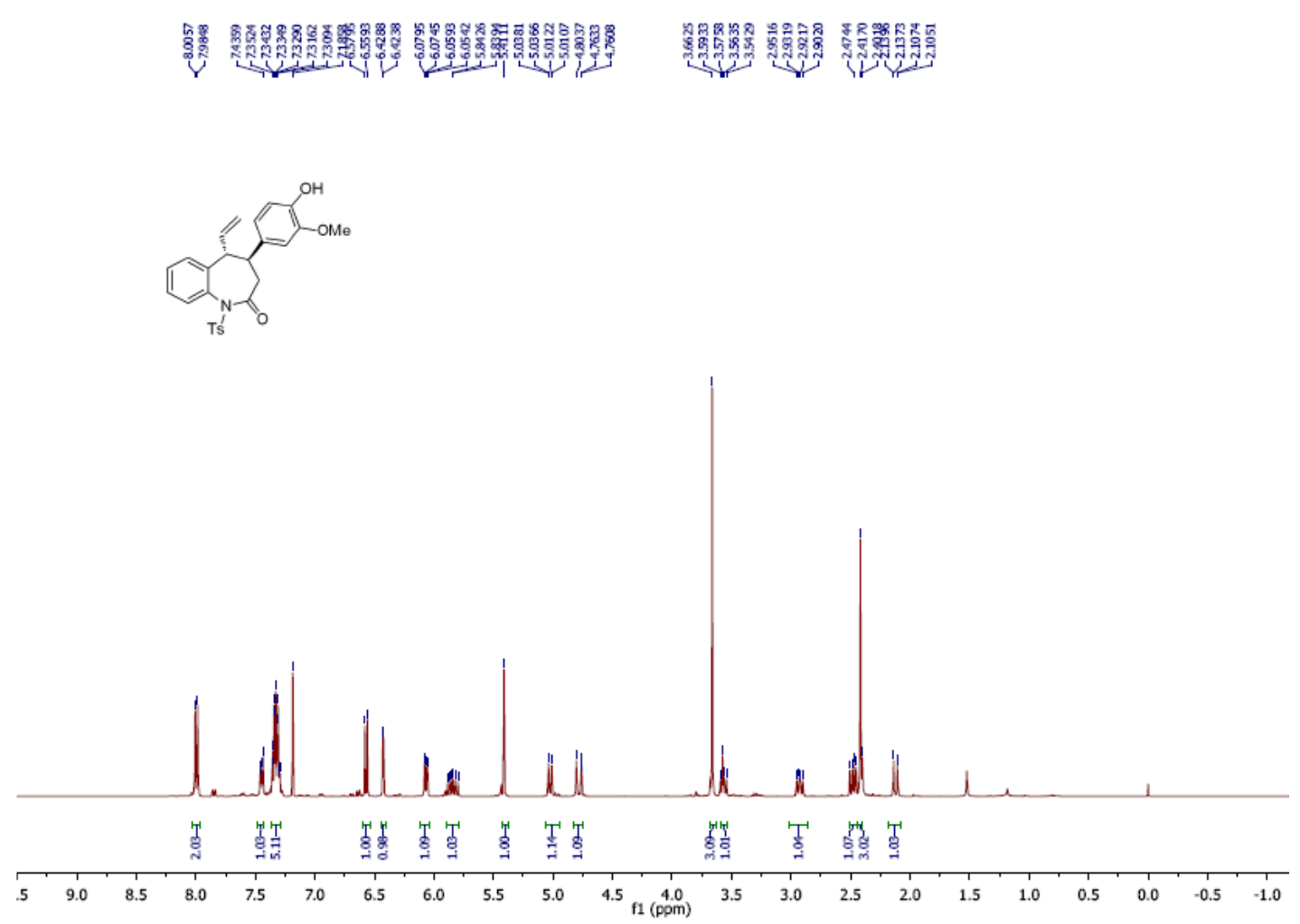

${ }^{13} \mathrm{C}$ NMR spectrum of $\mathbf{3 a j}$
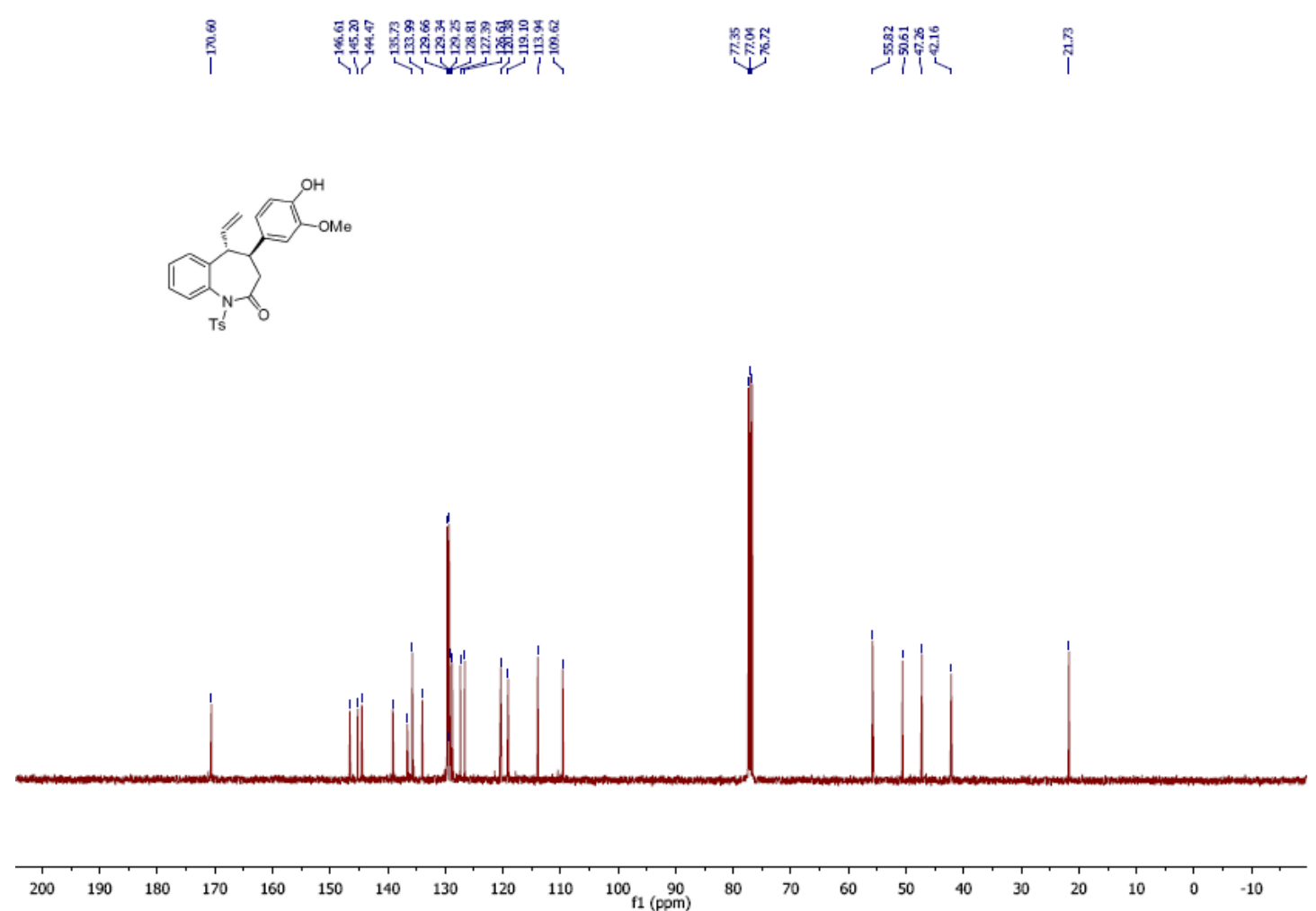
${ }^{1} \mathrm{H}$ NMR spectrum of $\mathbf{3 a k}$
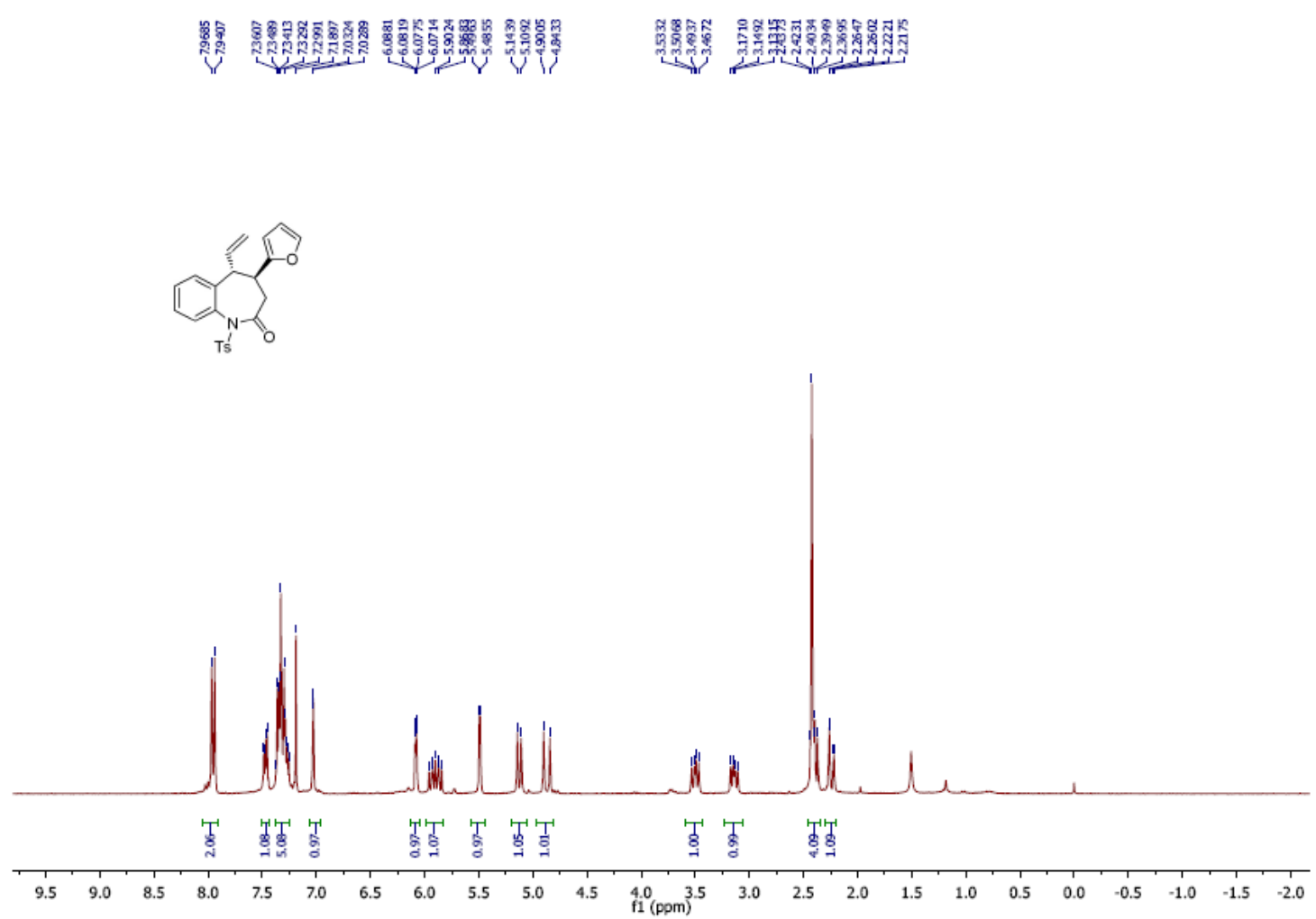

${ }^{13} \mathrm{C}$ NMR spectrum of $\mathbf{3 a k}$
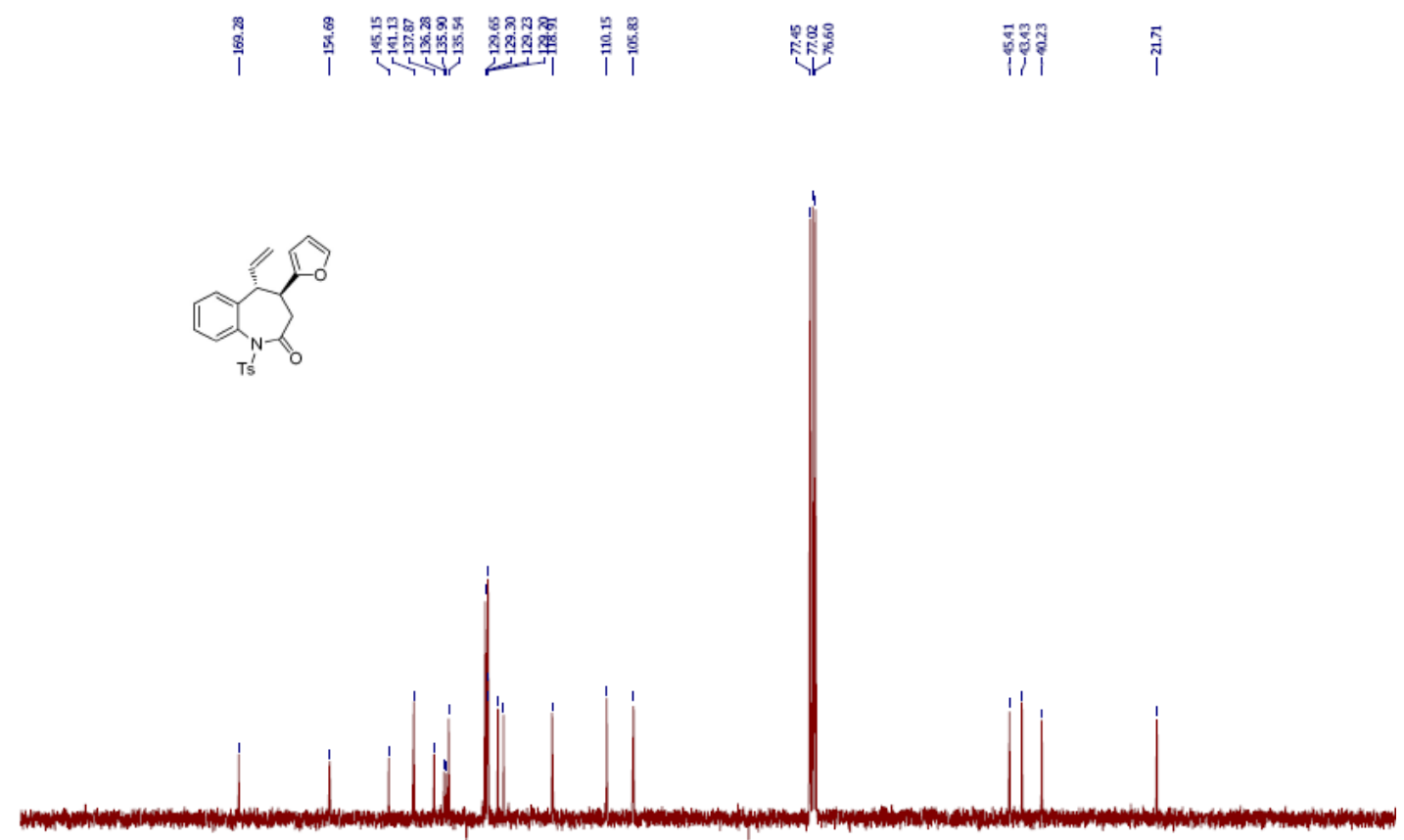

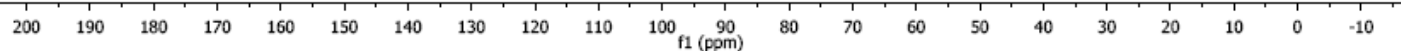


${ }^{1} \mathrm{H}$ NMR spectrum of 3al
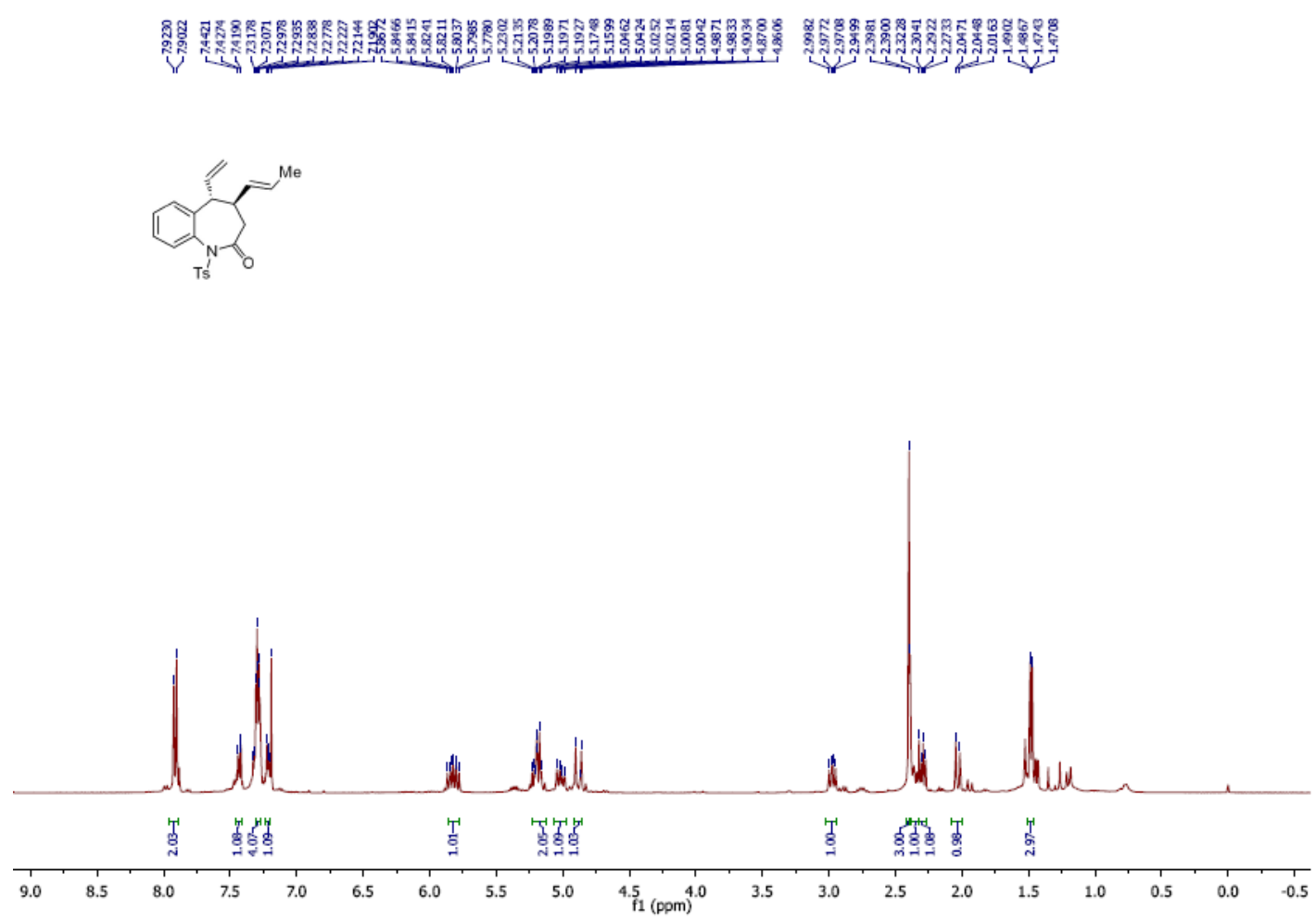

${ }^{13} \mathrm{C}$ NMR spectrum of 3al
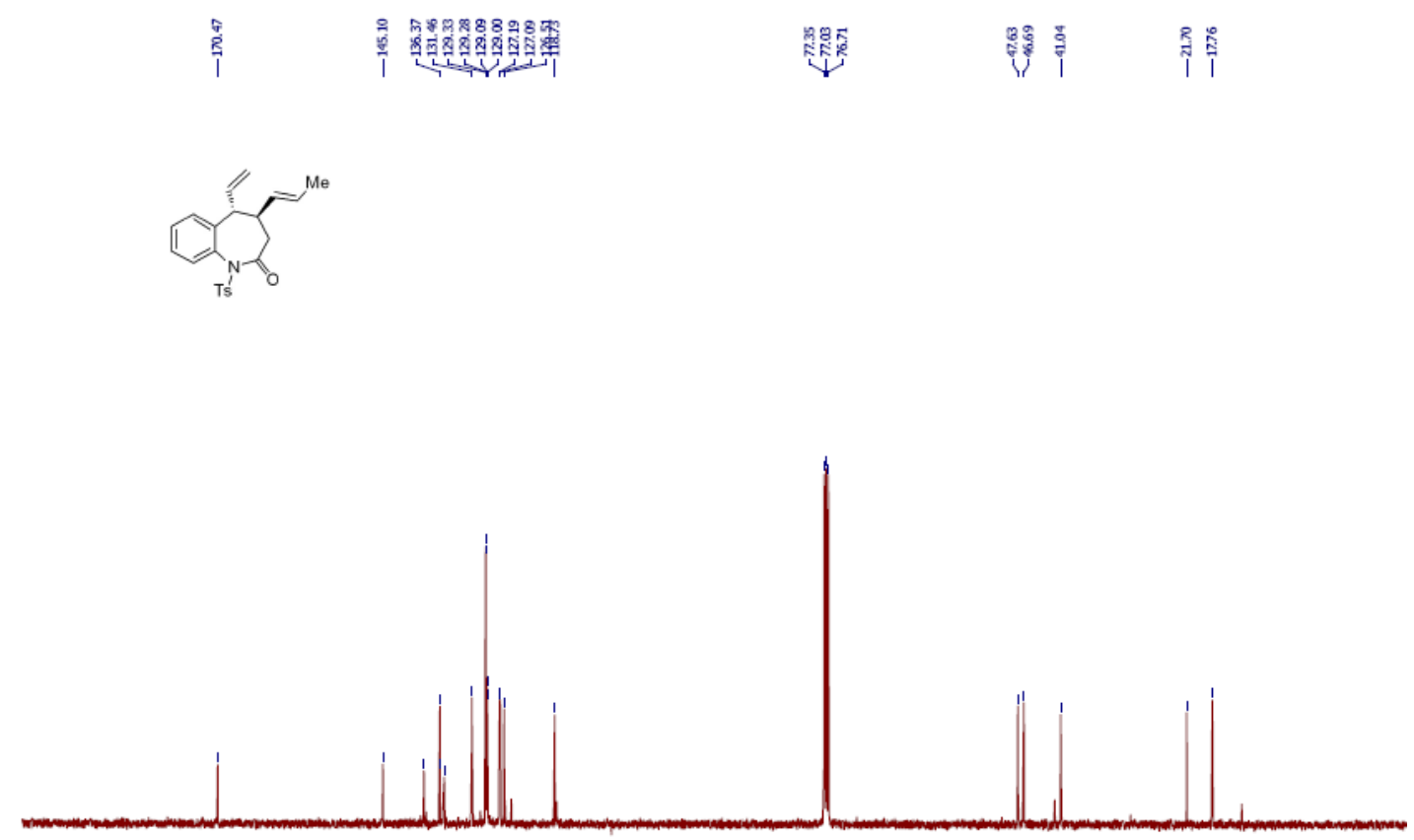

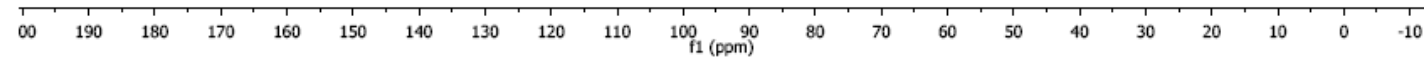


${ }^{1} \mathrm{H}$ NMR spectrum of $\mathbf{3 b a}$

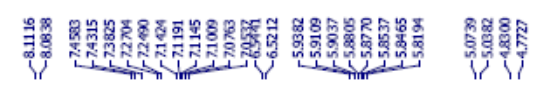

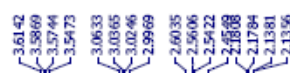
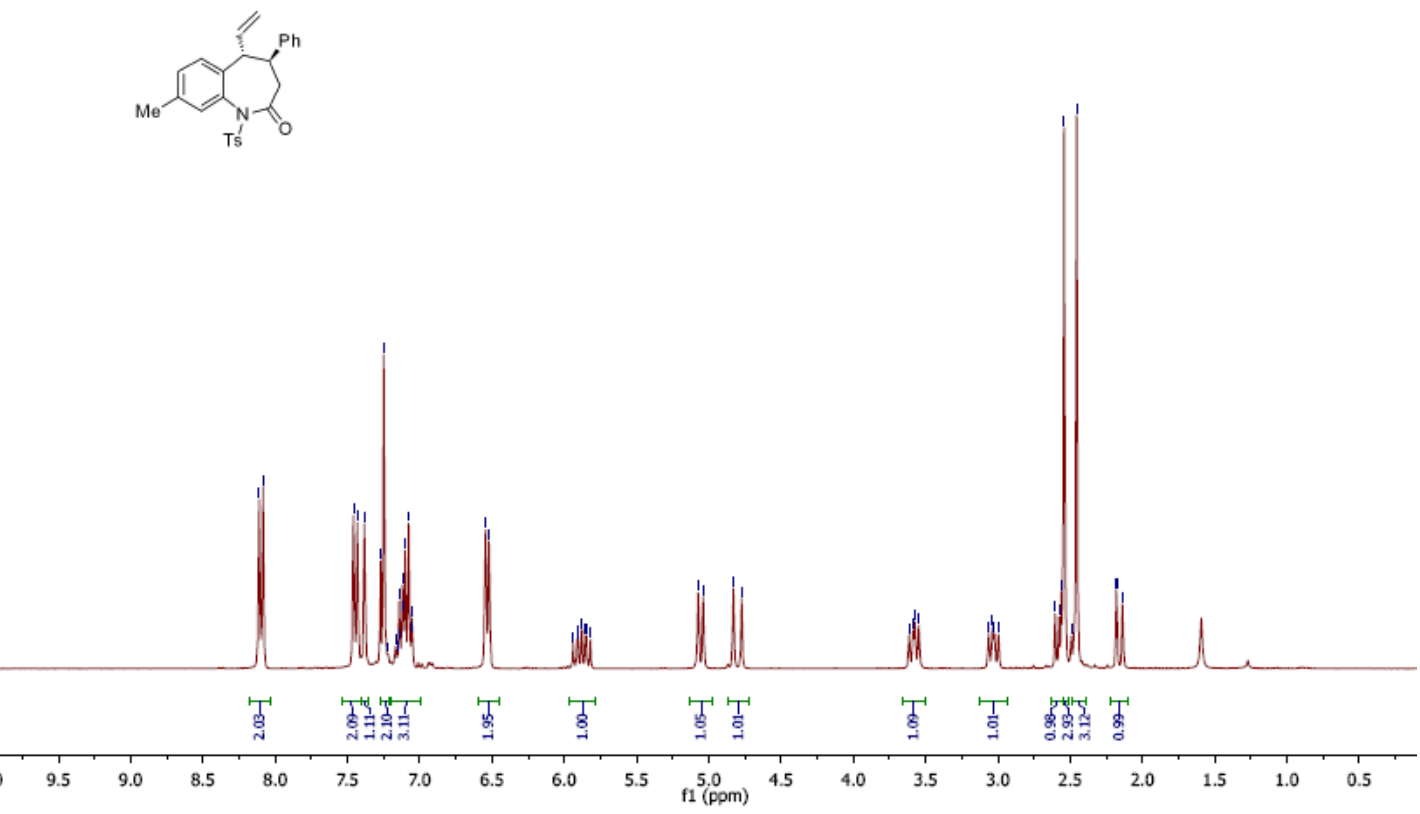

${ }^{13} \mathrm{C}$ NMR spectrum of $\mathbf{3 b a}$

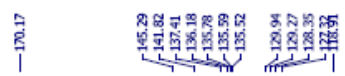
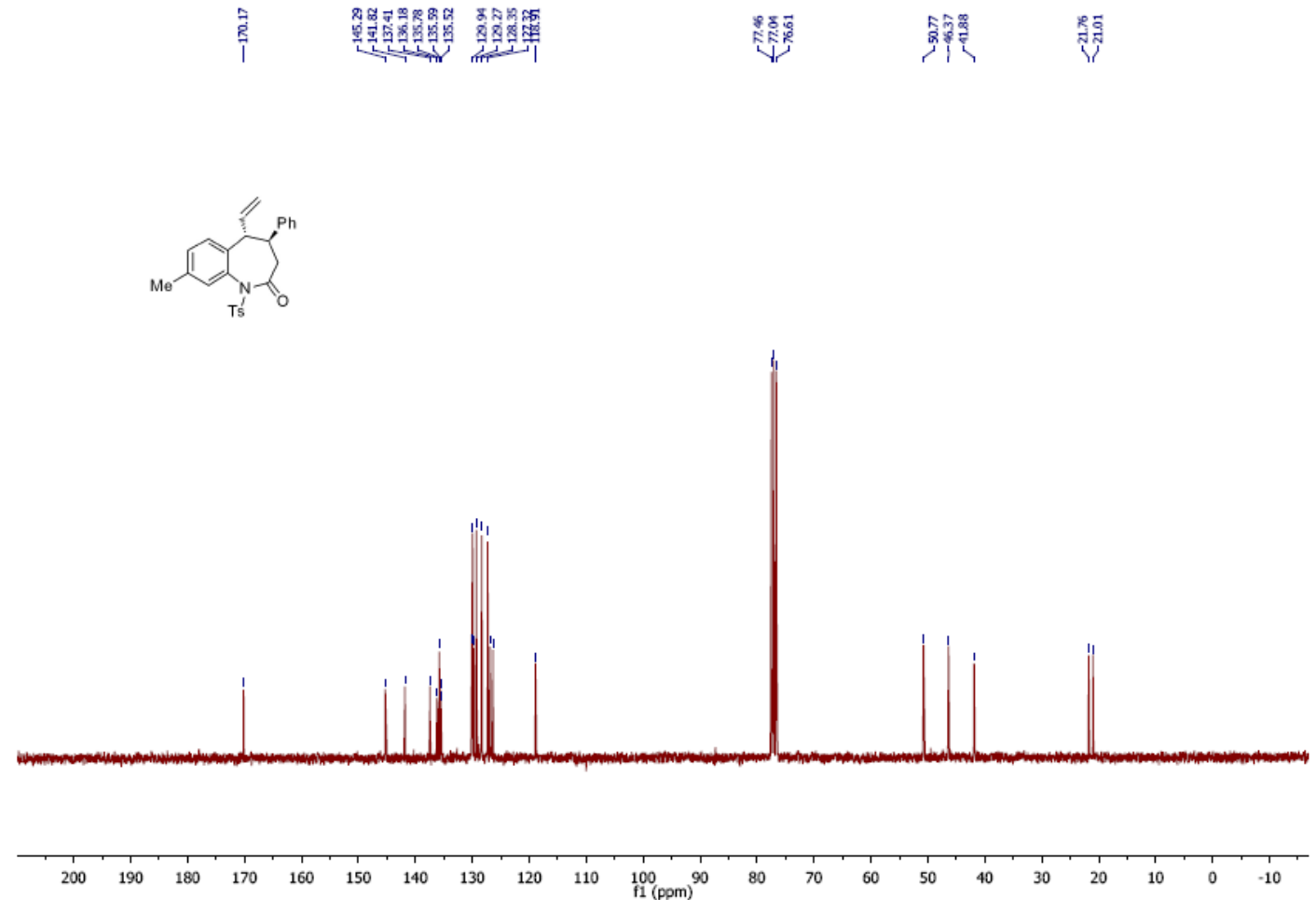
${ }^{1} \mathrm{H}$ NMR spectrum of 3ca
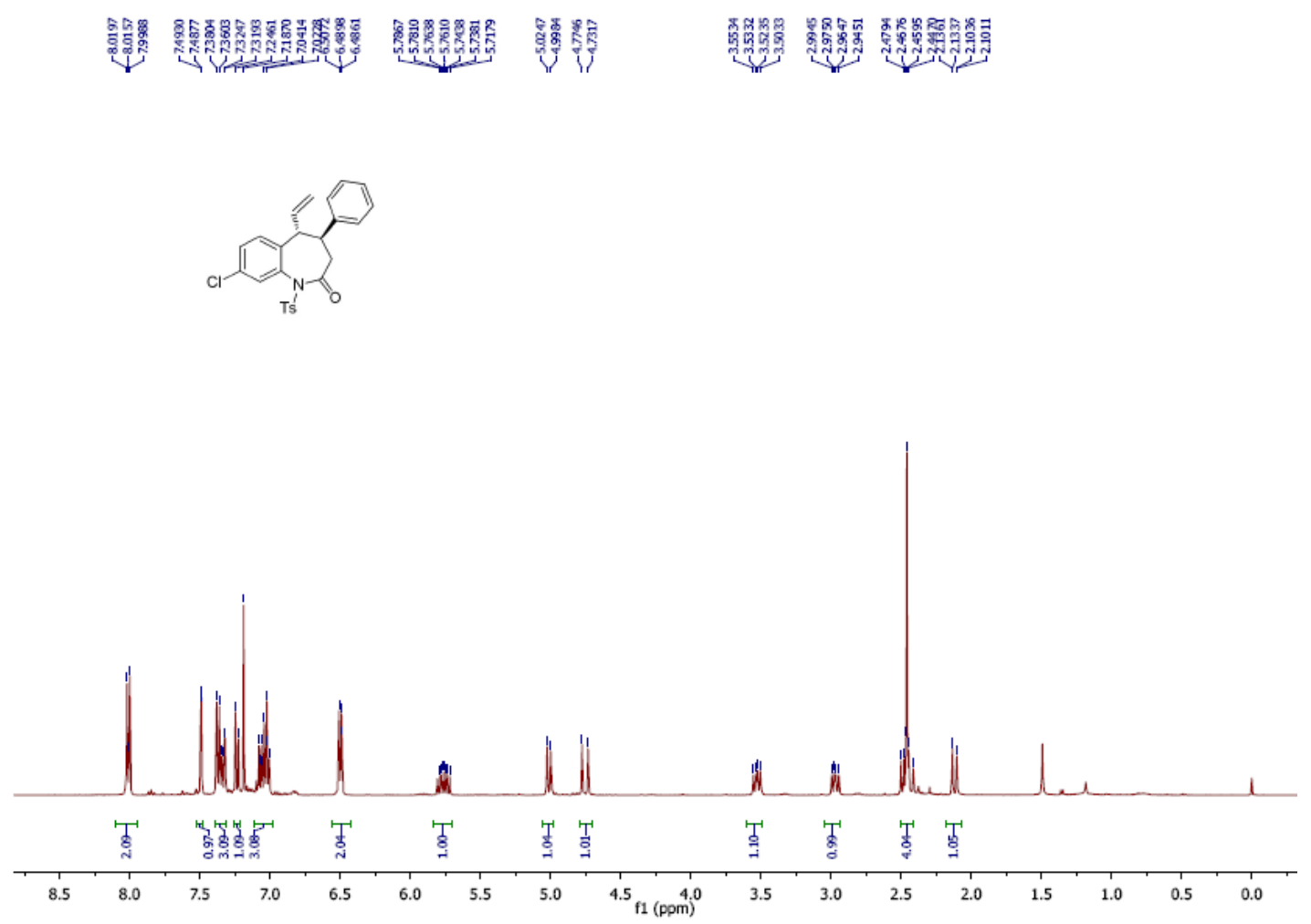

${ }^{13} \mathrm{C}$ NMR spectrum of $\mathbf{3 c a}$

5.
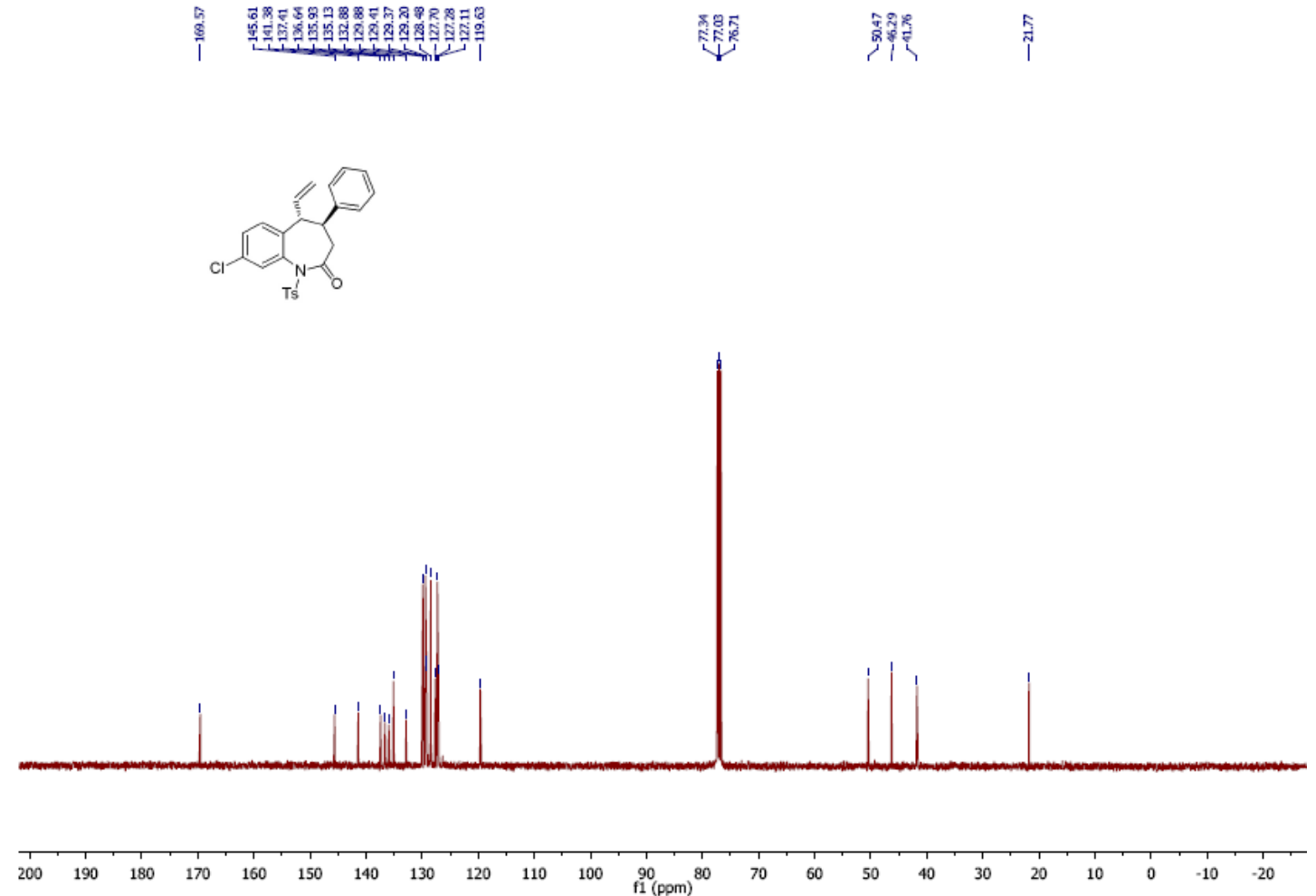
${ }^{1} \mathrm{H}$ NMR spectrum of $\mathbf{3 d a}$
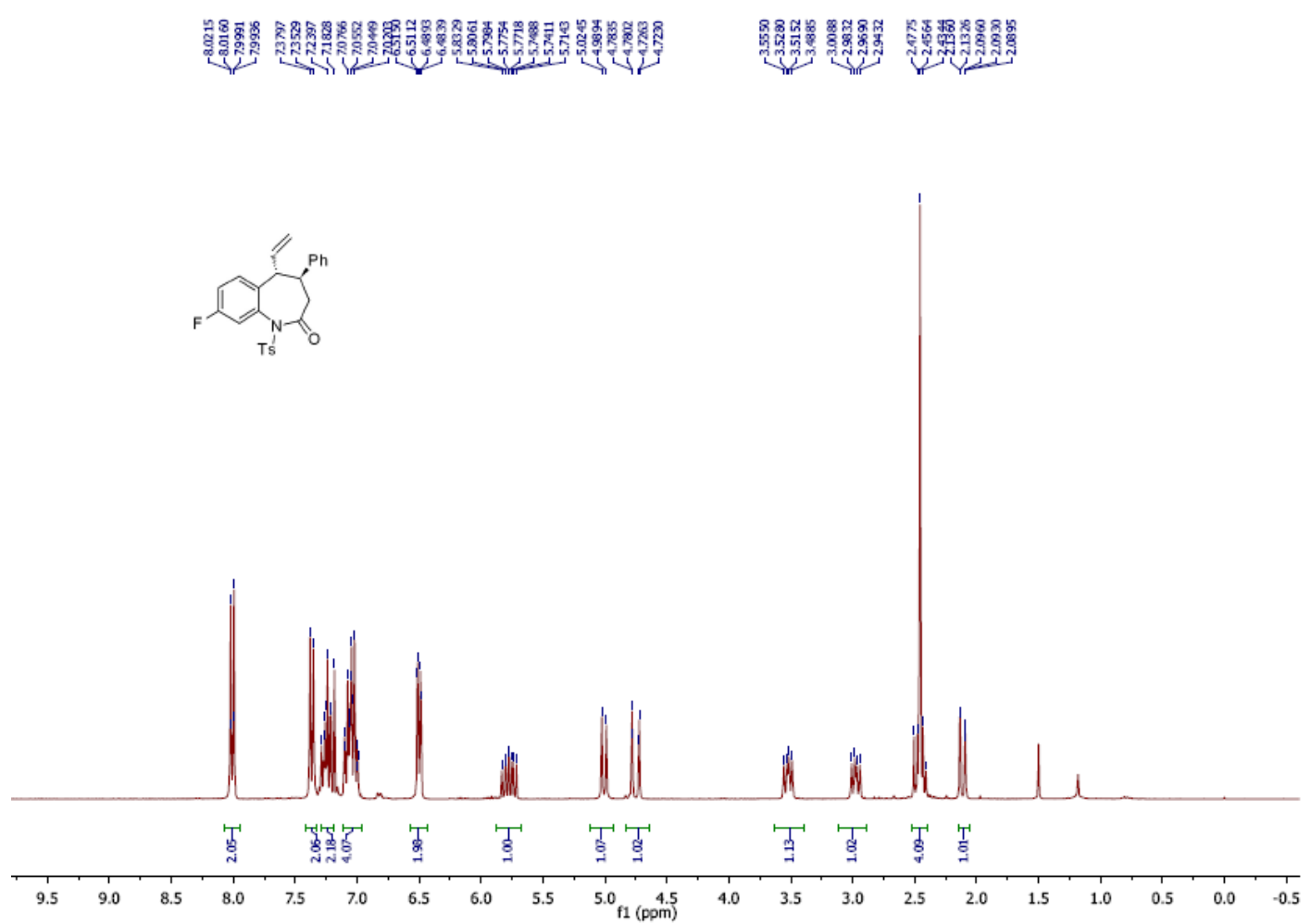

${ }^{13} \mathrm{C}$ NMR spectrum of $\mathbf{3 d a}$
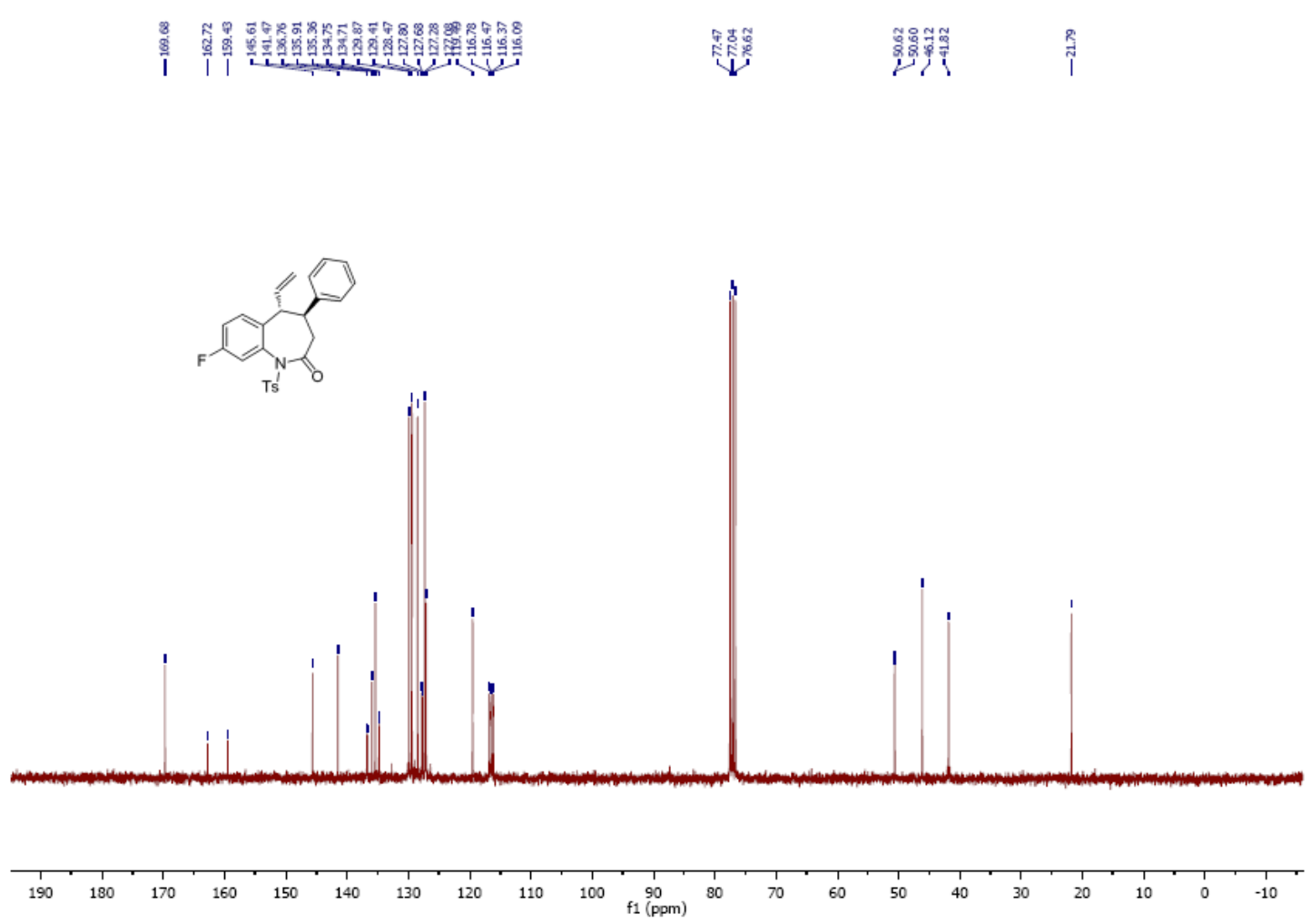
${ }^{1} \mathrm{H}$ NMR spectrum of $\mathbf{3 e a}$
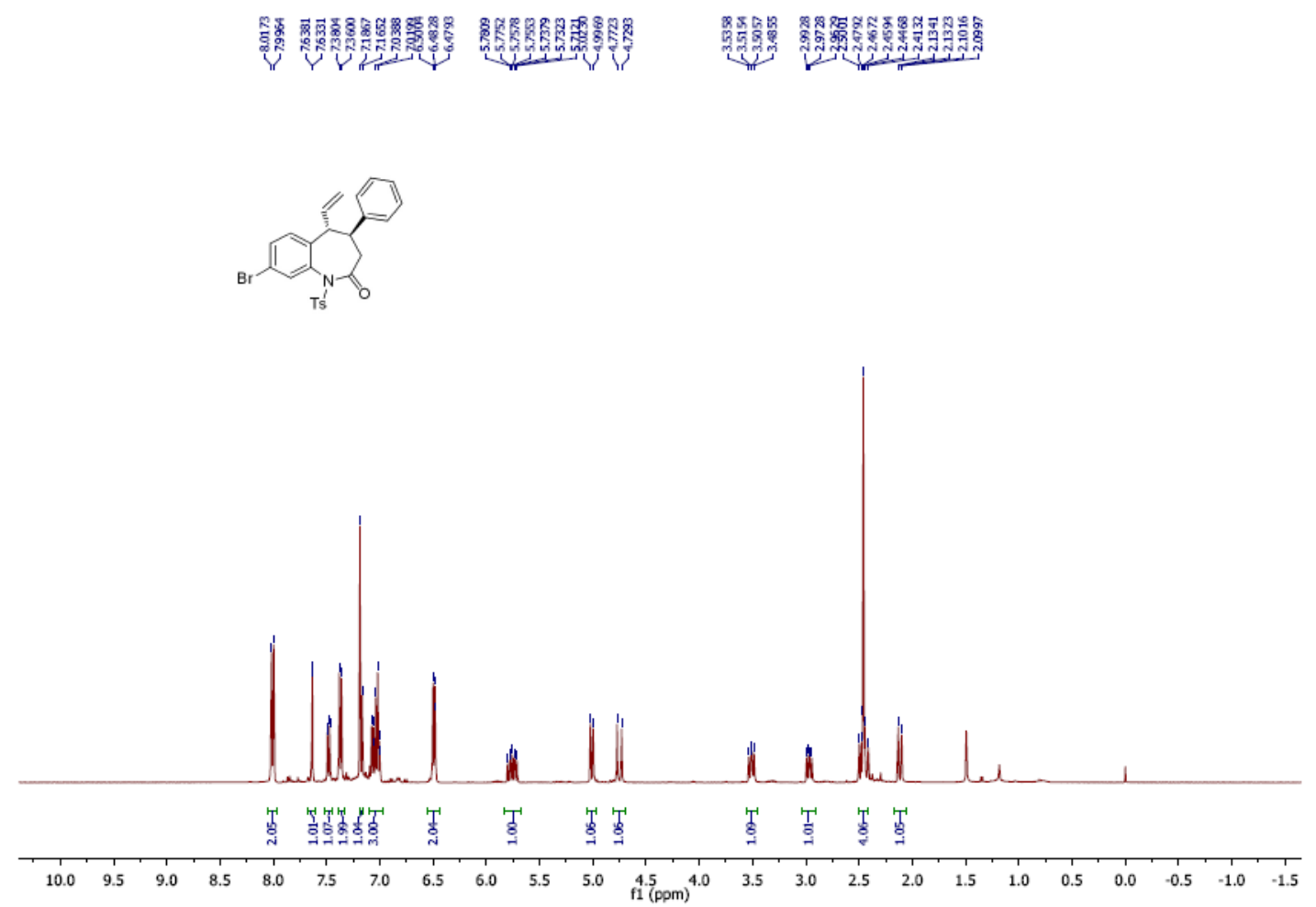

${ }^{13} \mathrm{C}$ NMR spectrum of $\mathbf{3 e a}$
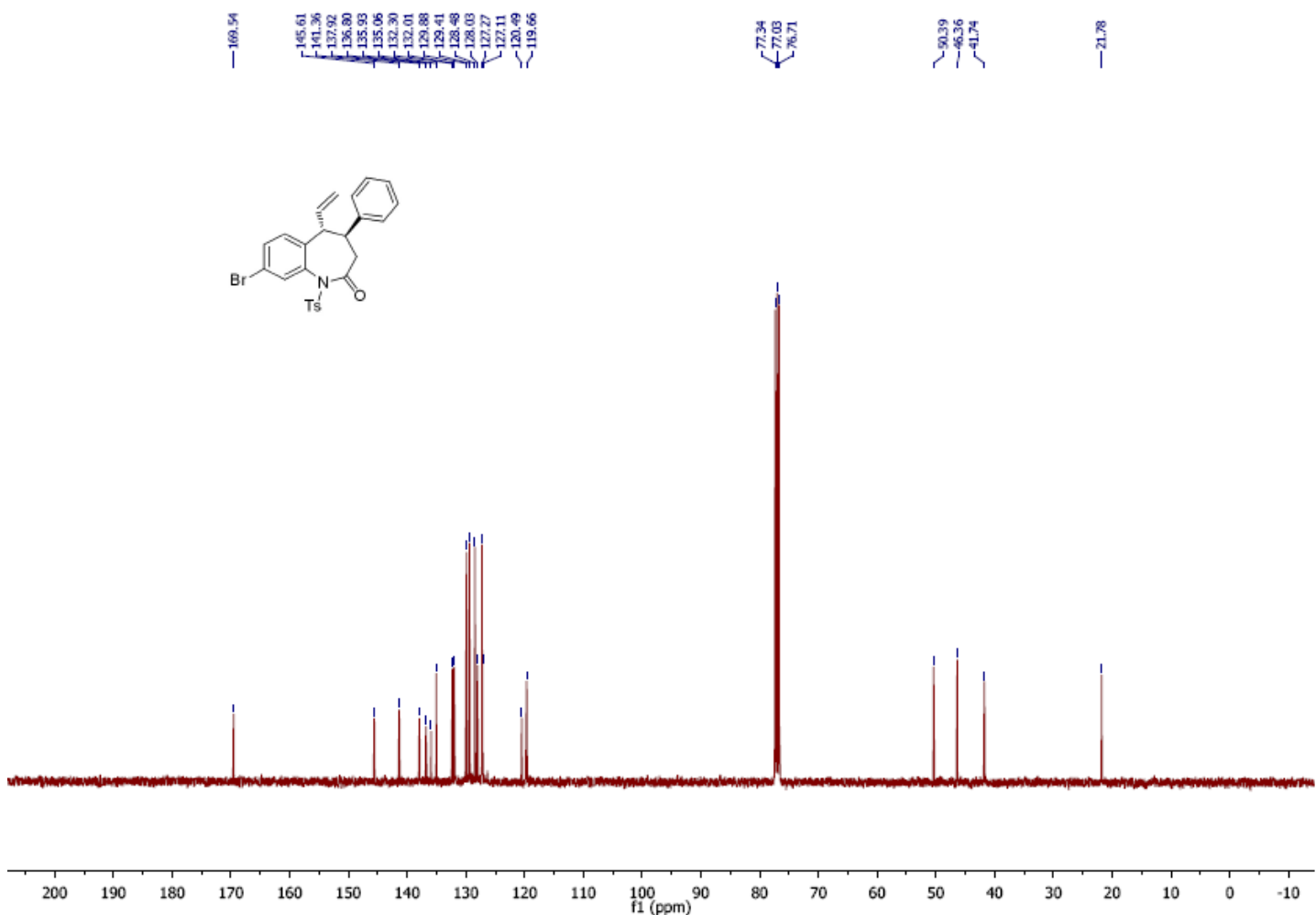
${ }^{1} \mathrm{H}$ NMR spectrum of $\mathbf{3 f a}$
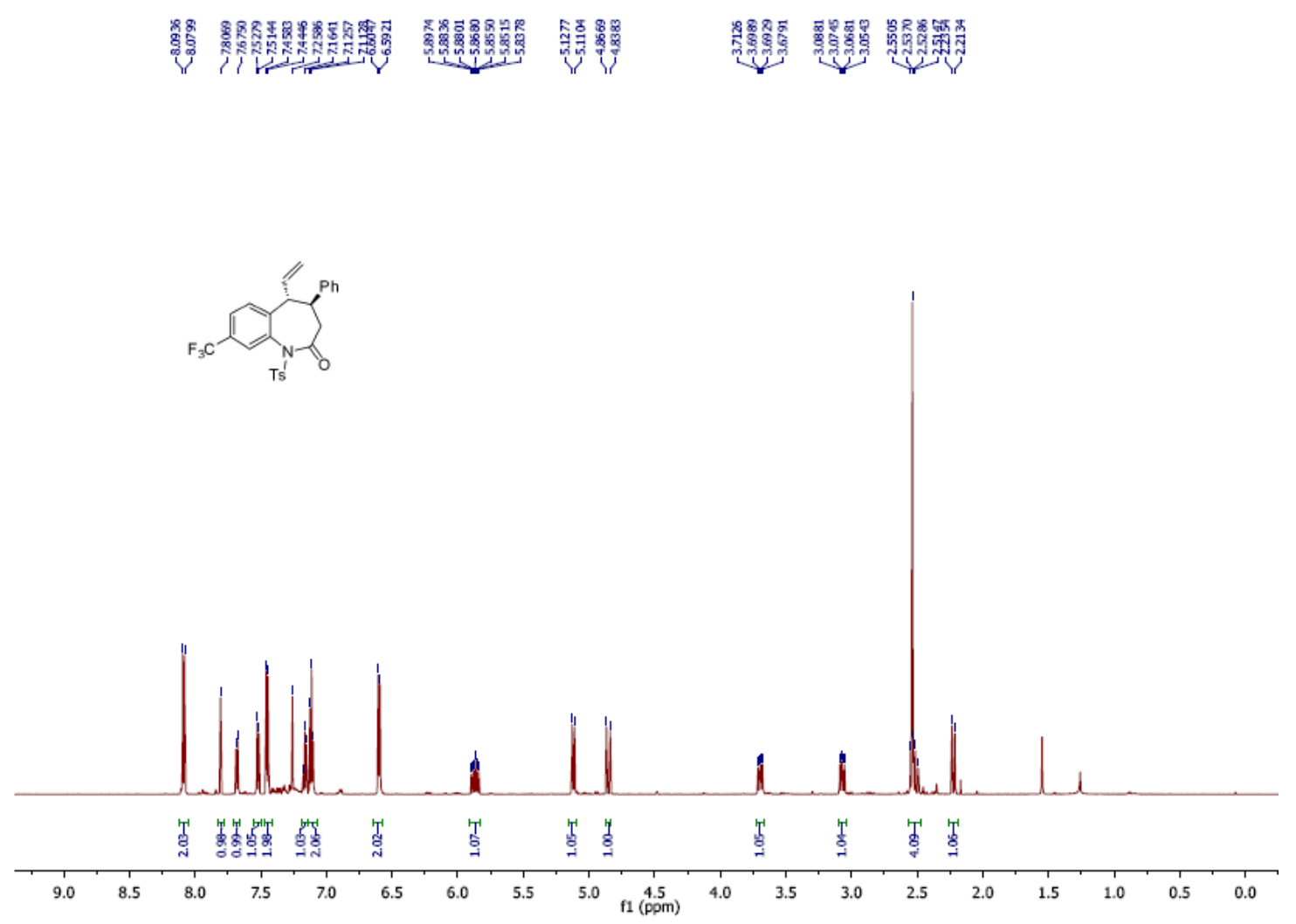

${ }^{13} \mathrm{C}$ NMR spectrum of $\mathbf{3 f a}$

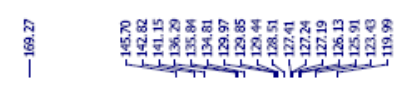

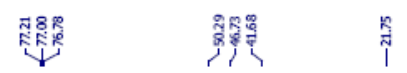
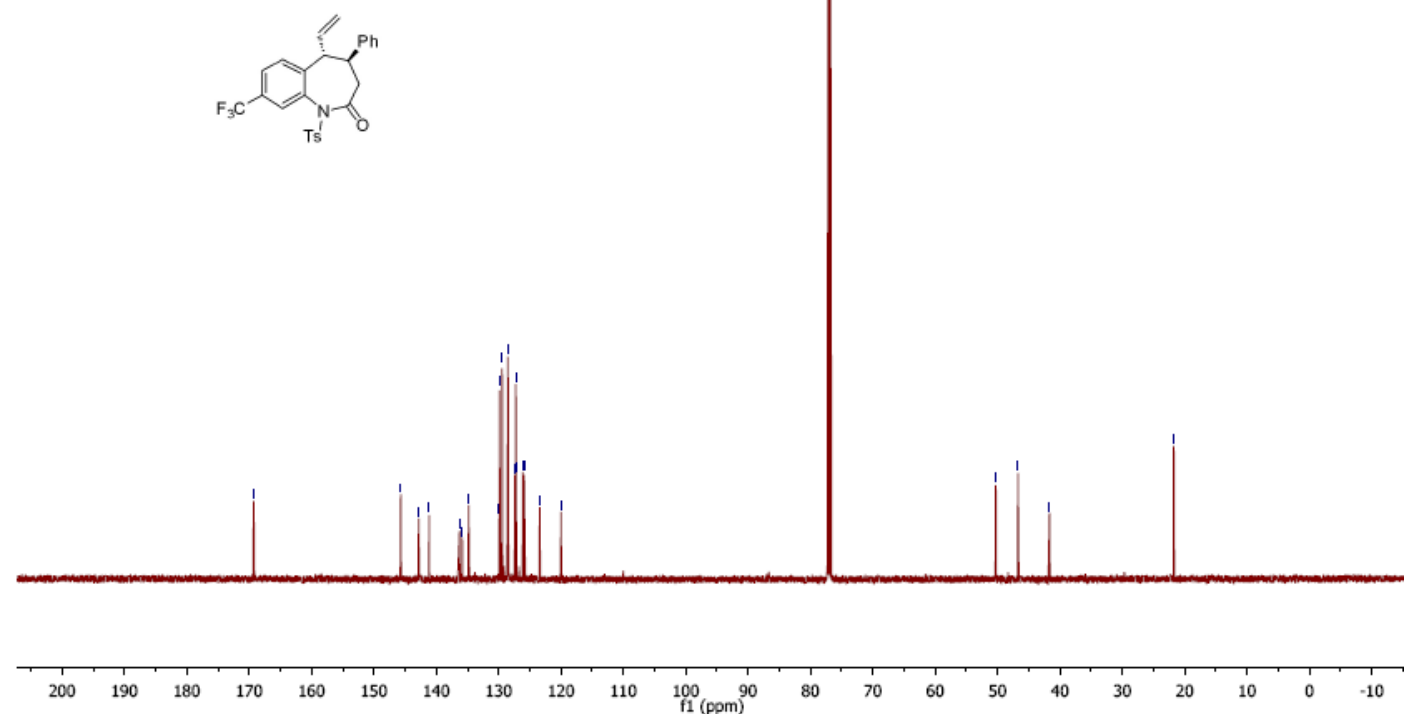
${ }^{1} \mathrm{H}$ NMR spectrum of $3 g a$
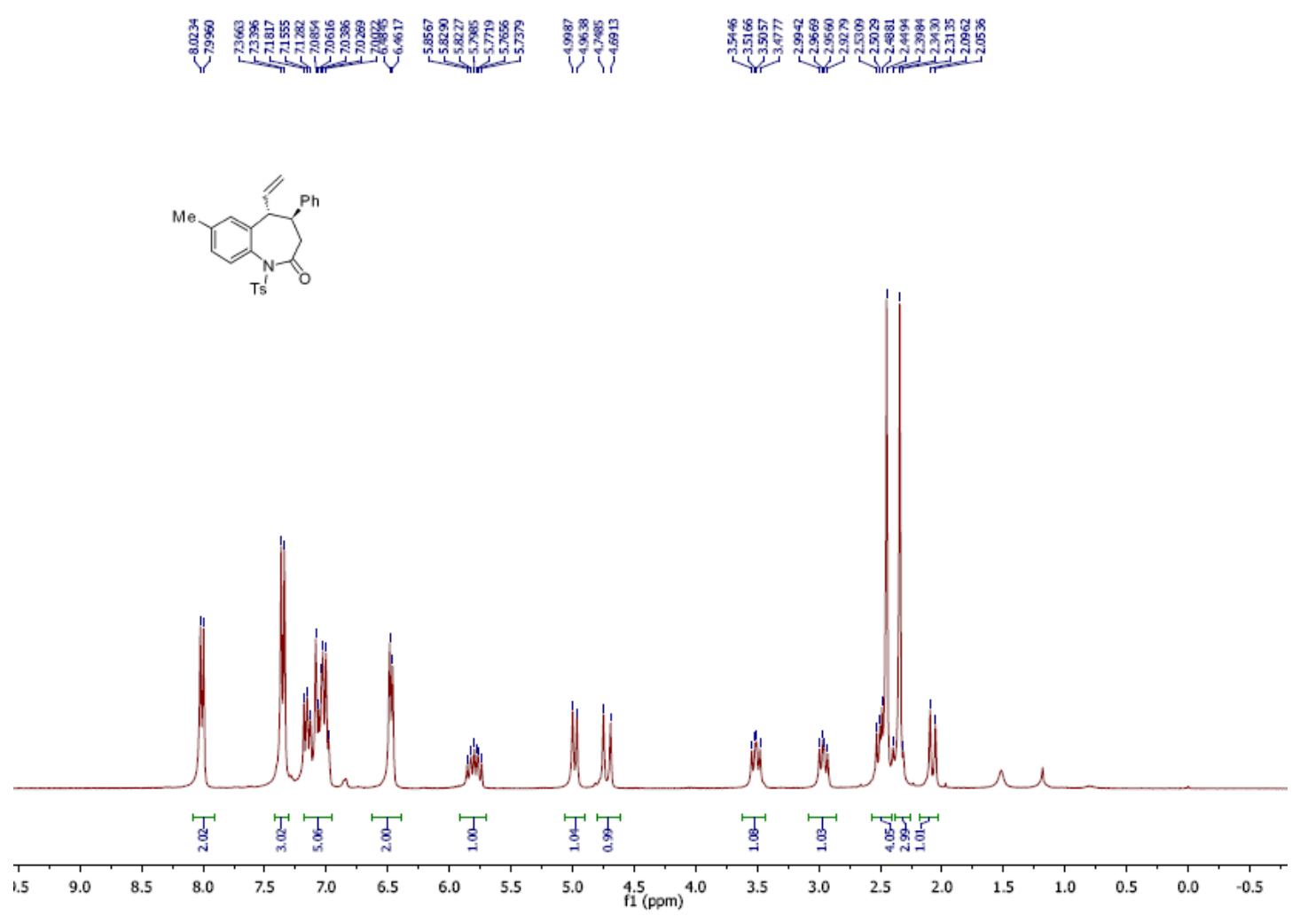

${ }^{13} \mathrm{C}$ NMR spectrum of 3 ga

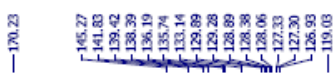

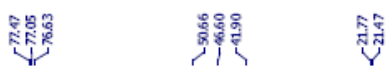
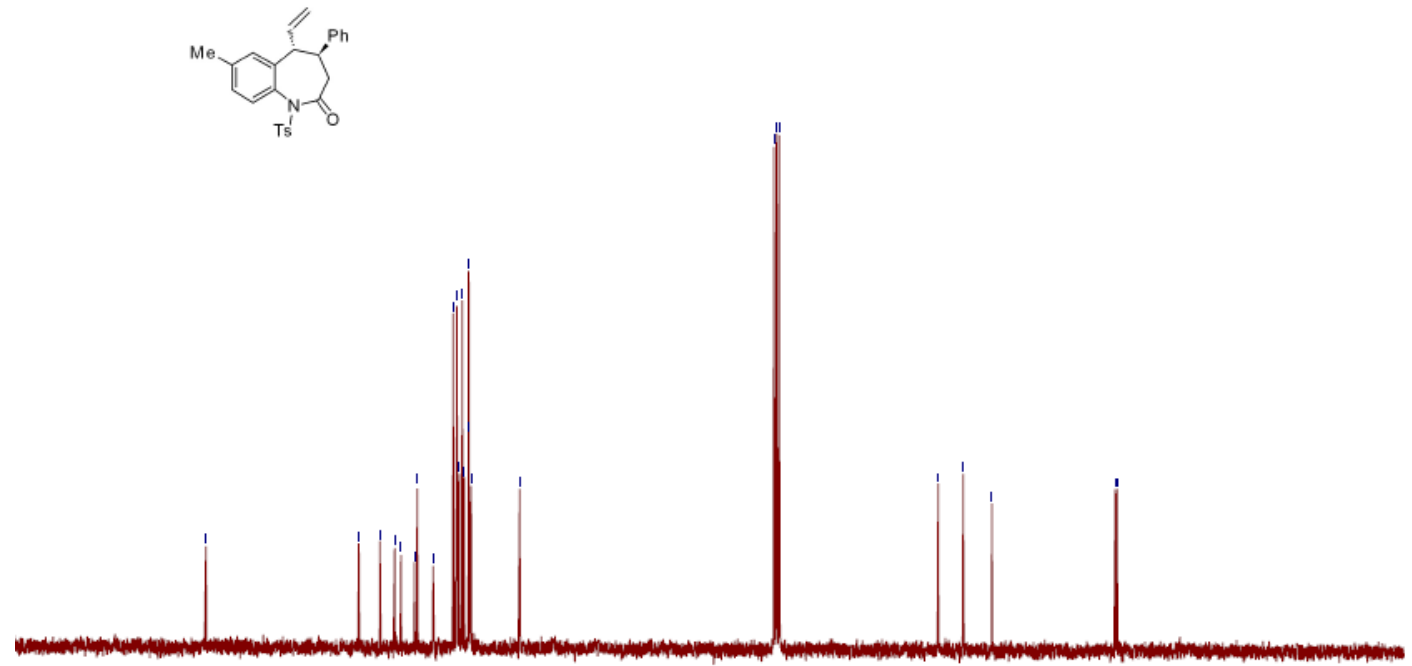

$\begin{array}{llllllllllllllllllllllllllllll}100 & 190 & 180 & 170 & 160 & 150 & 140 & 130 & 120 & 110 & 100 & 90 & 80 & 70 & 60 & 50 & 40 & 30 & 20 & 10 & 0 & -10 & -20\end{array}$ 
${ }^{1} \mathrm{H}$ NMR spectrum of 3 ha
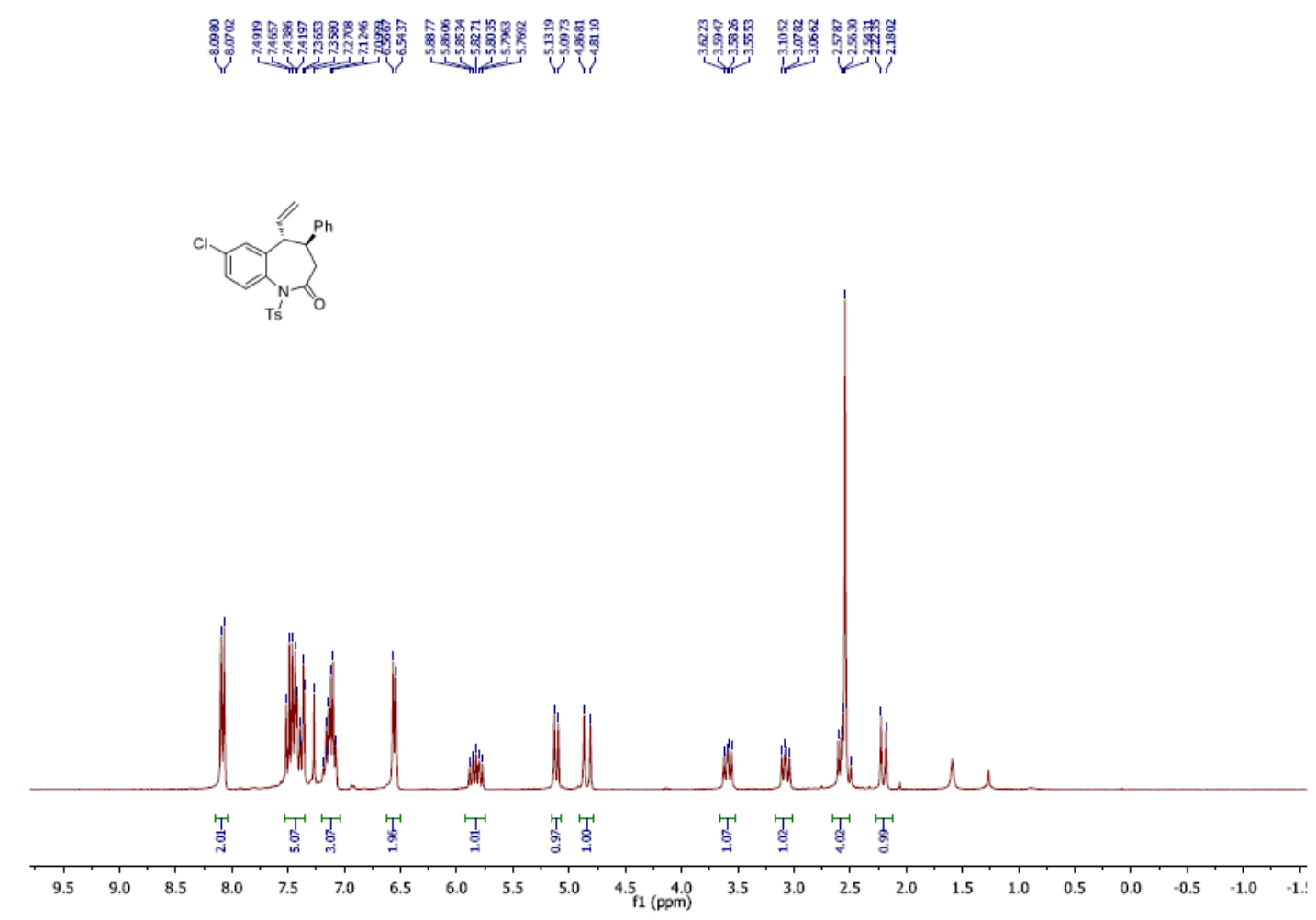

${ }^{13} \mathrm{C}$ NMR spectrum of $\mathbf{3 h a}$

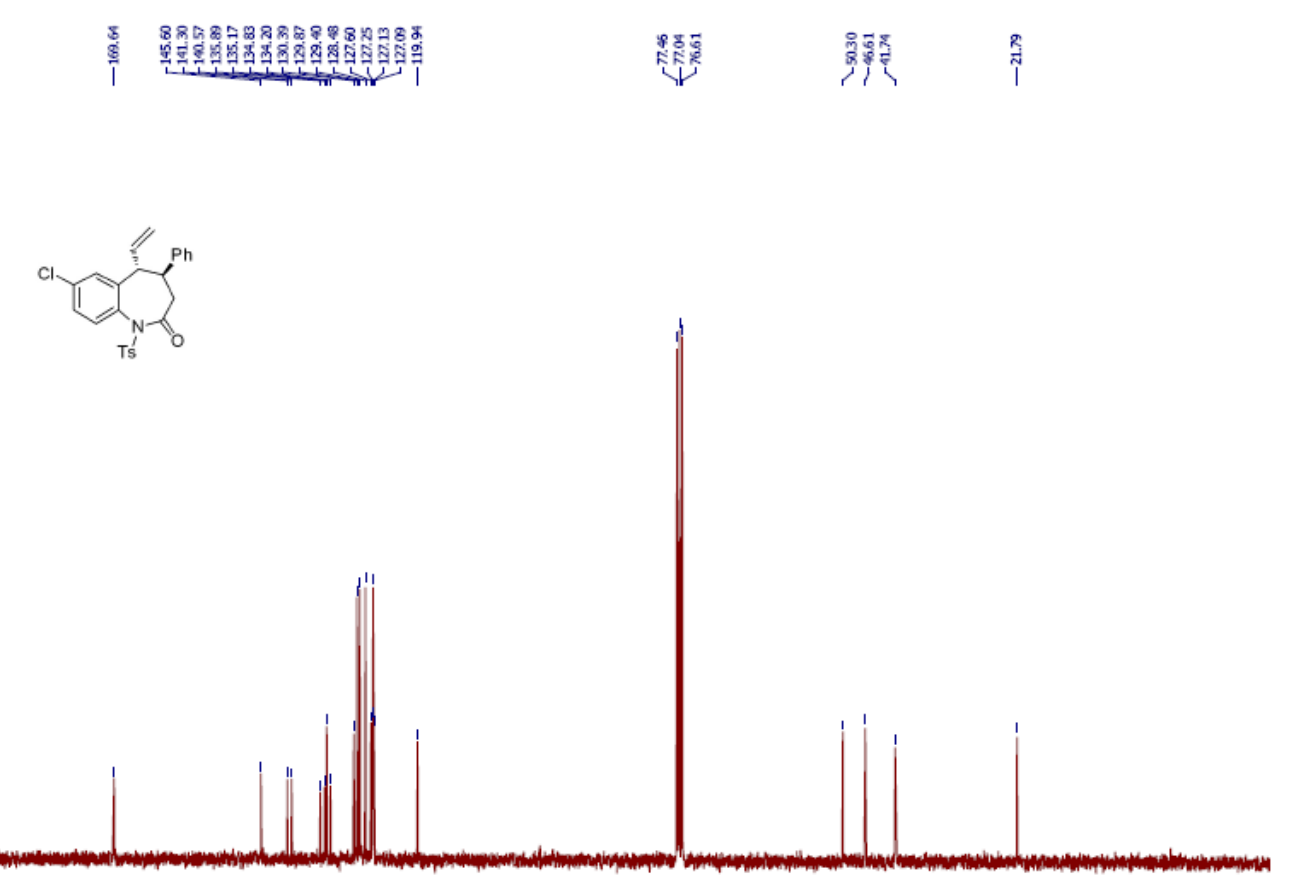

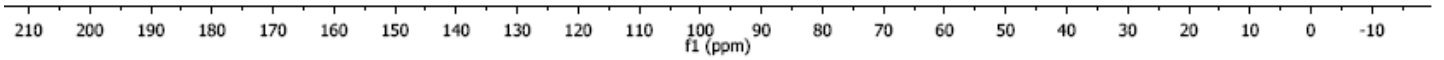


${ }^{1} \mathrm{H}$ NMR spectrum of 3ia
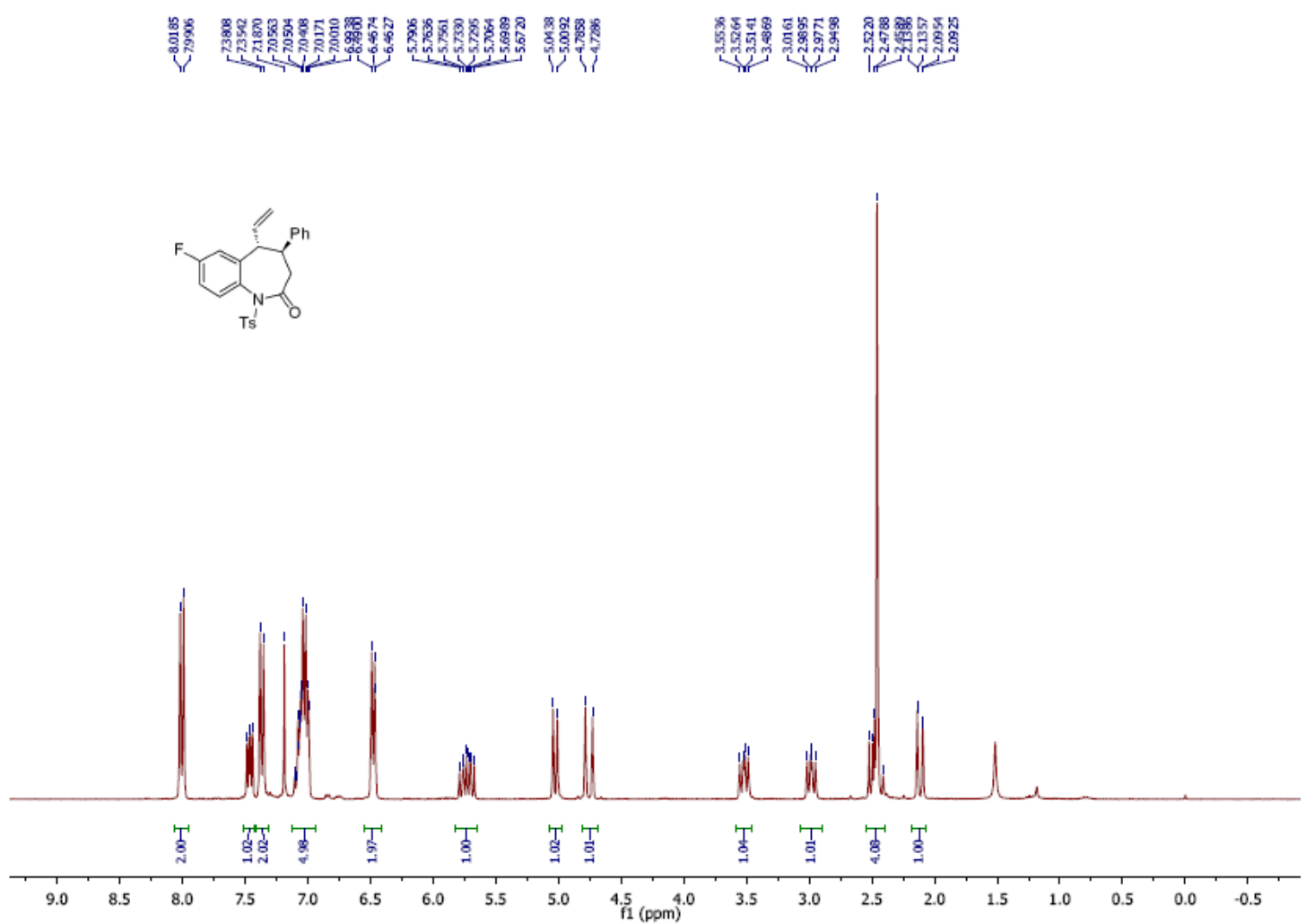

${ }^{13} \mathrm{C}$ NMR spectrum of $\mathbf{3 i a}$

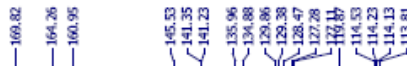

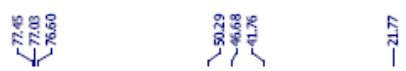
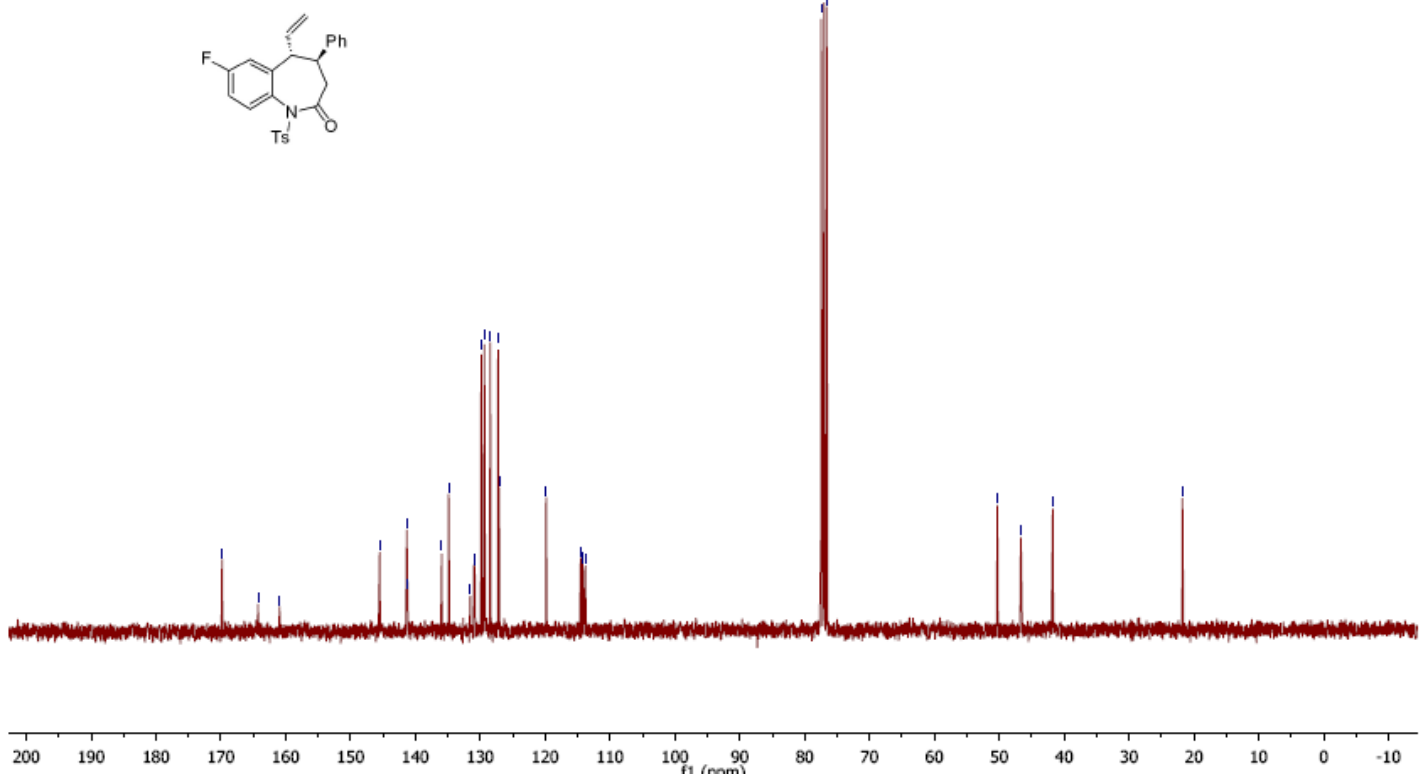
${ }^{1} \mathrm{H}$ NMR spectrum of $\mathbf{3 j a}$
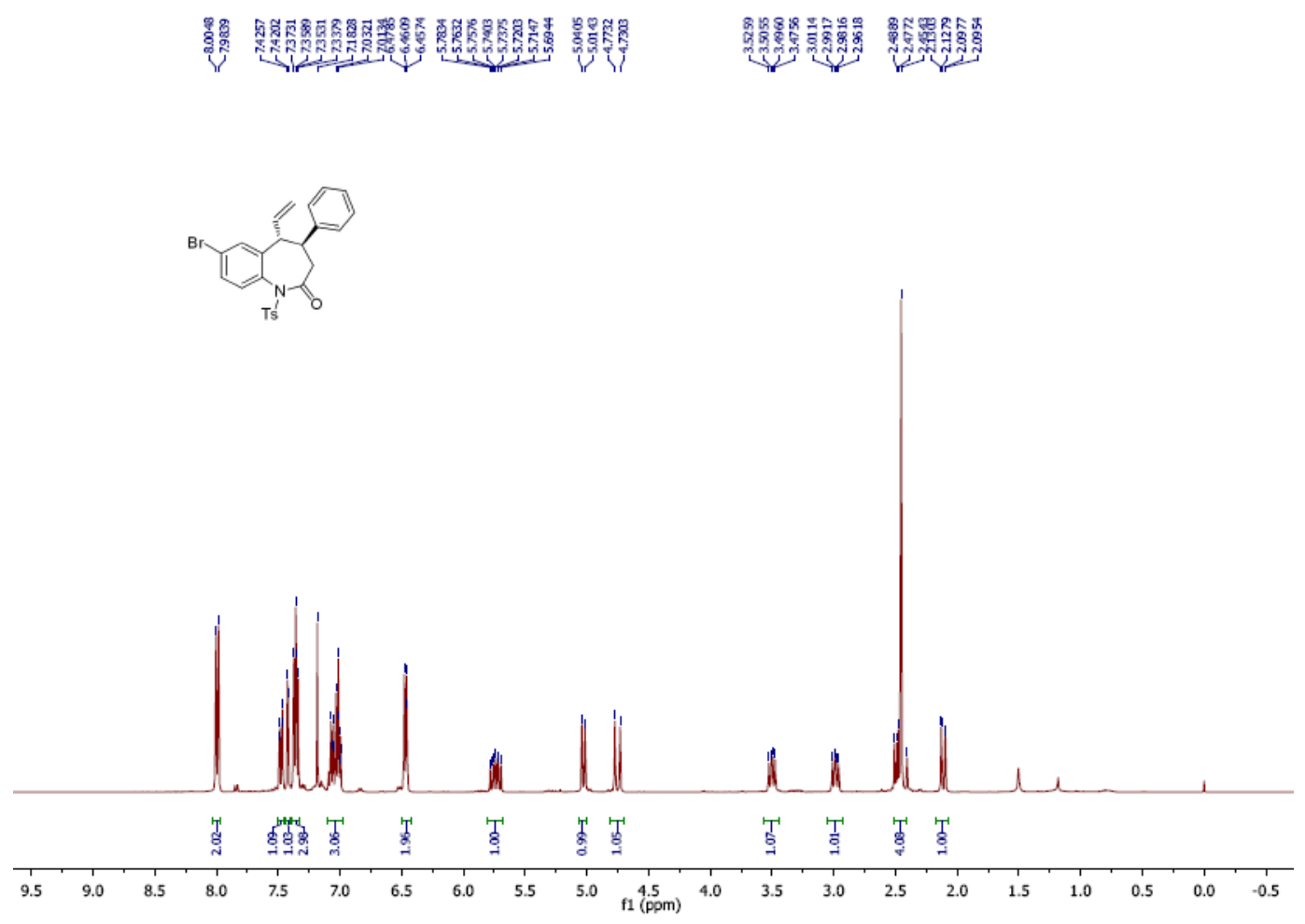

${ }^{13} \mathrm{C}$ NMR spectrum of $\mathbf{3 j a}$

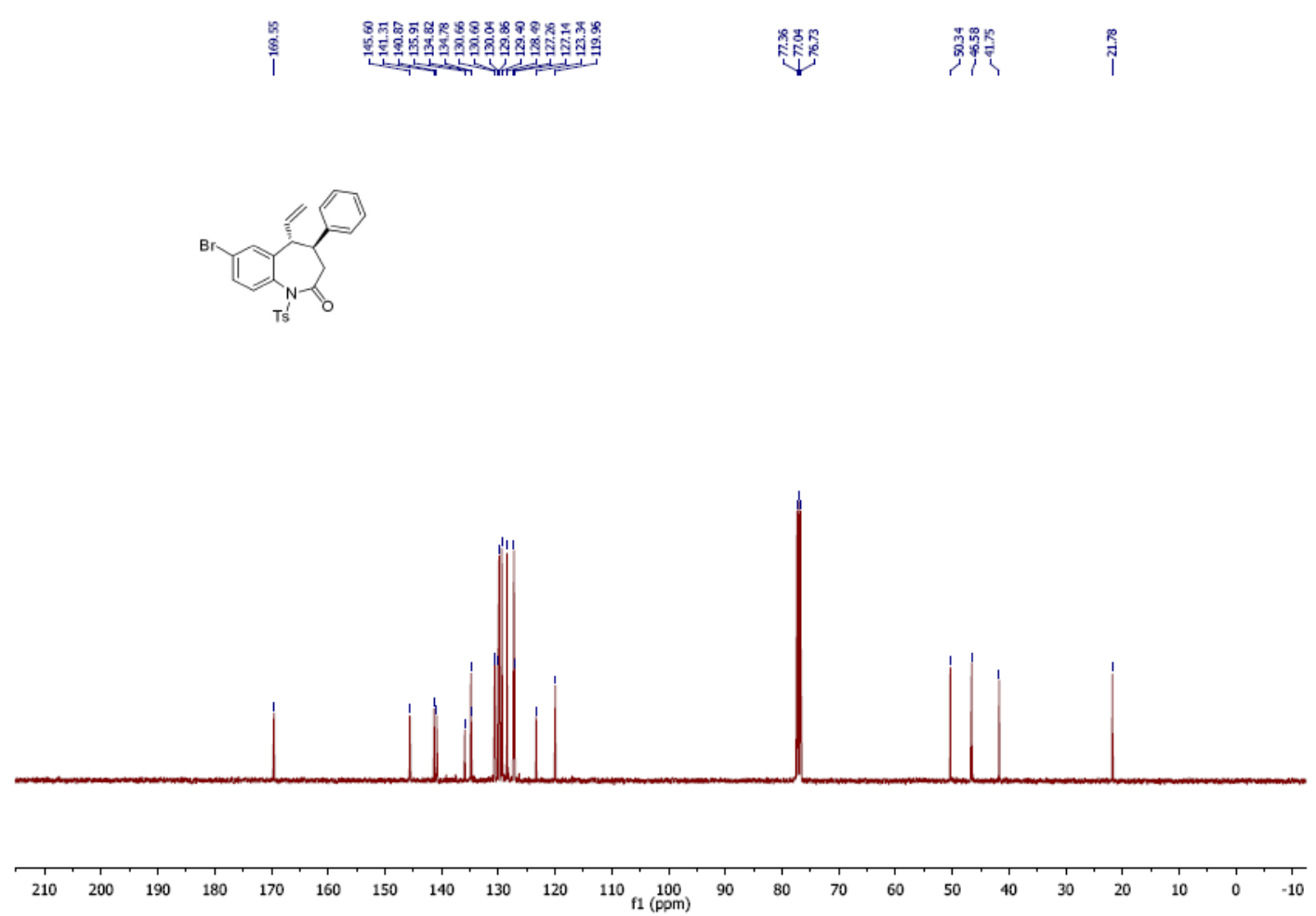


${ }^{1} \mathrm{H}$ NMR spectrum of $\mathbf{3 k a}$
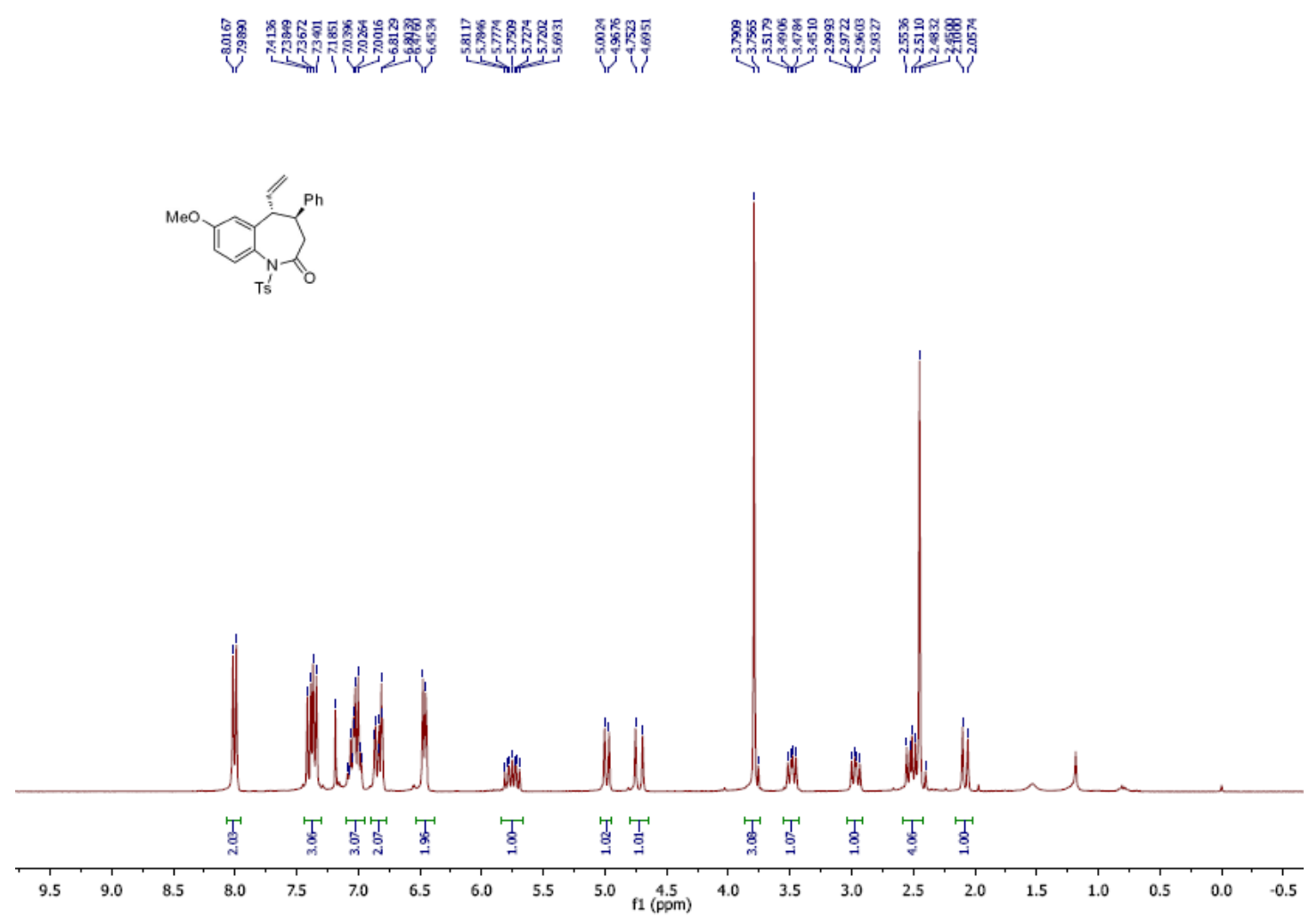

${ }^{13} \mathrm{C}$ NMR spectrum of $\mathbf{3 k a}$
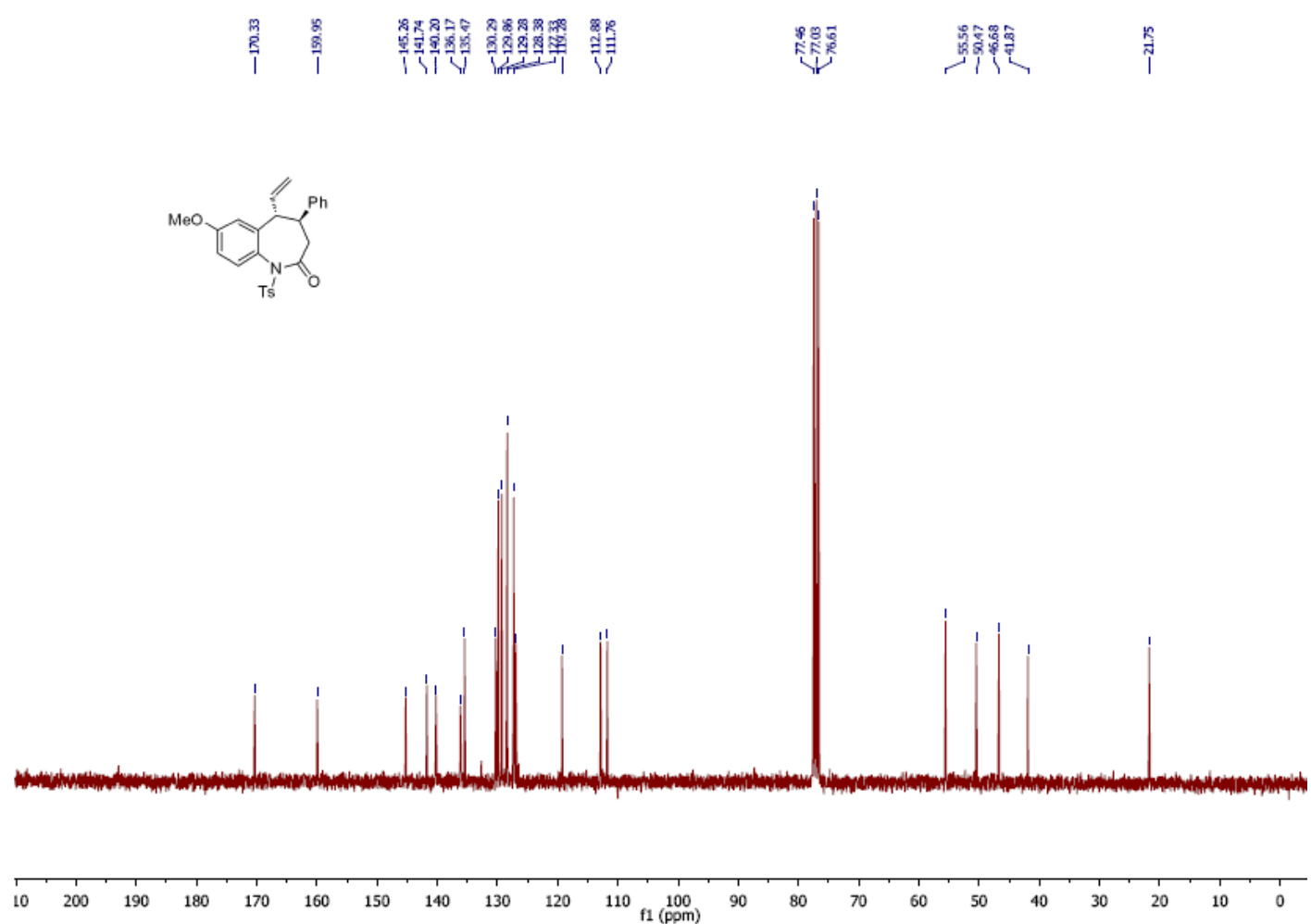
${ }^{1} \mathrm{H}$ NMR spectrum of $\mathbf{3 b k}$

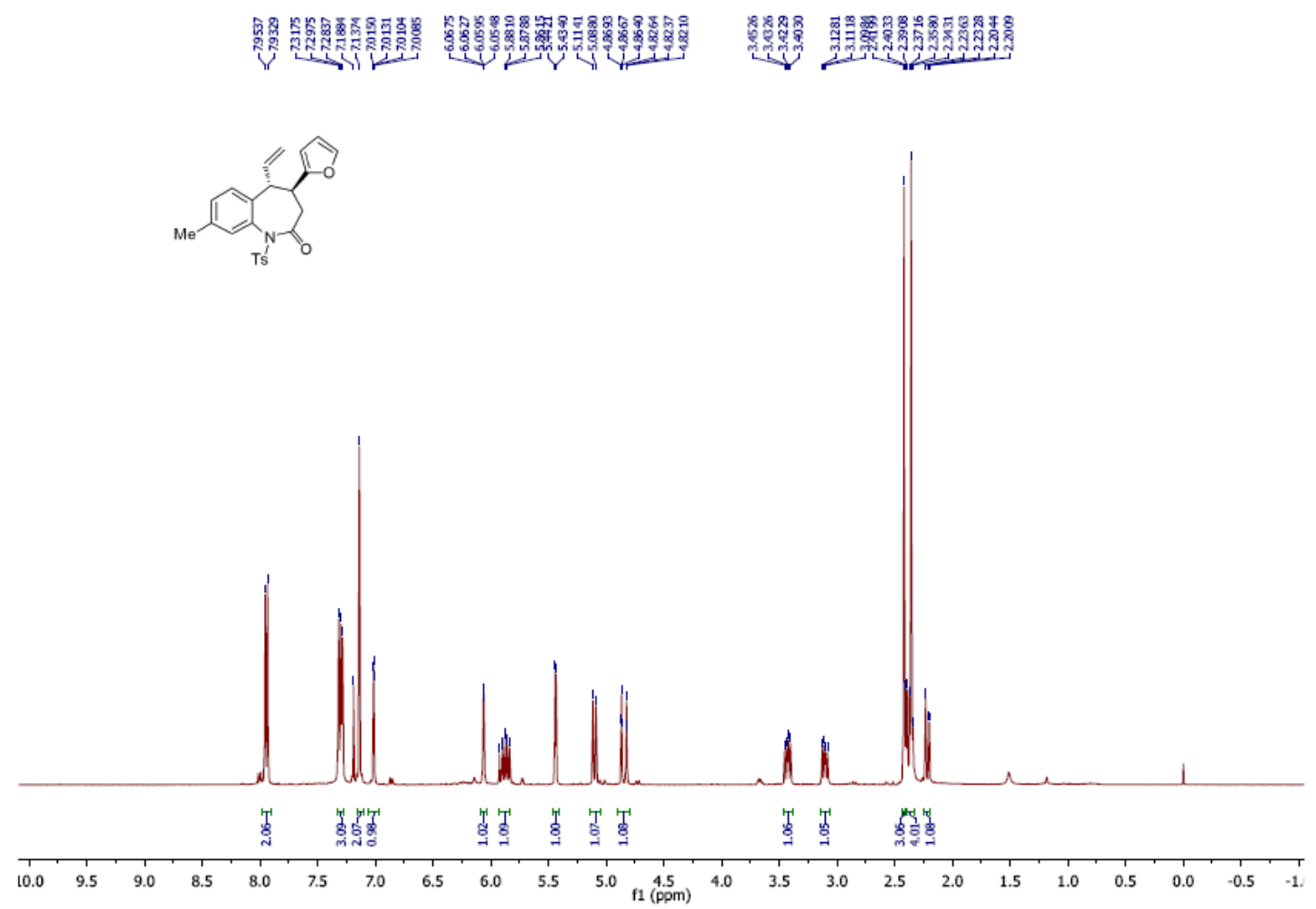

${ }^{13} \mathrm{C}$ NMR spectrum of $\mathbf{3 b k}$
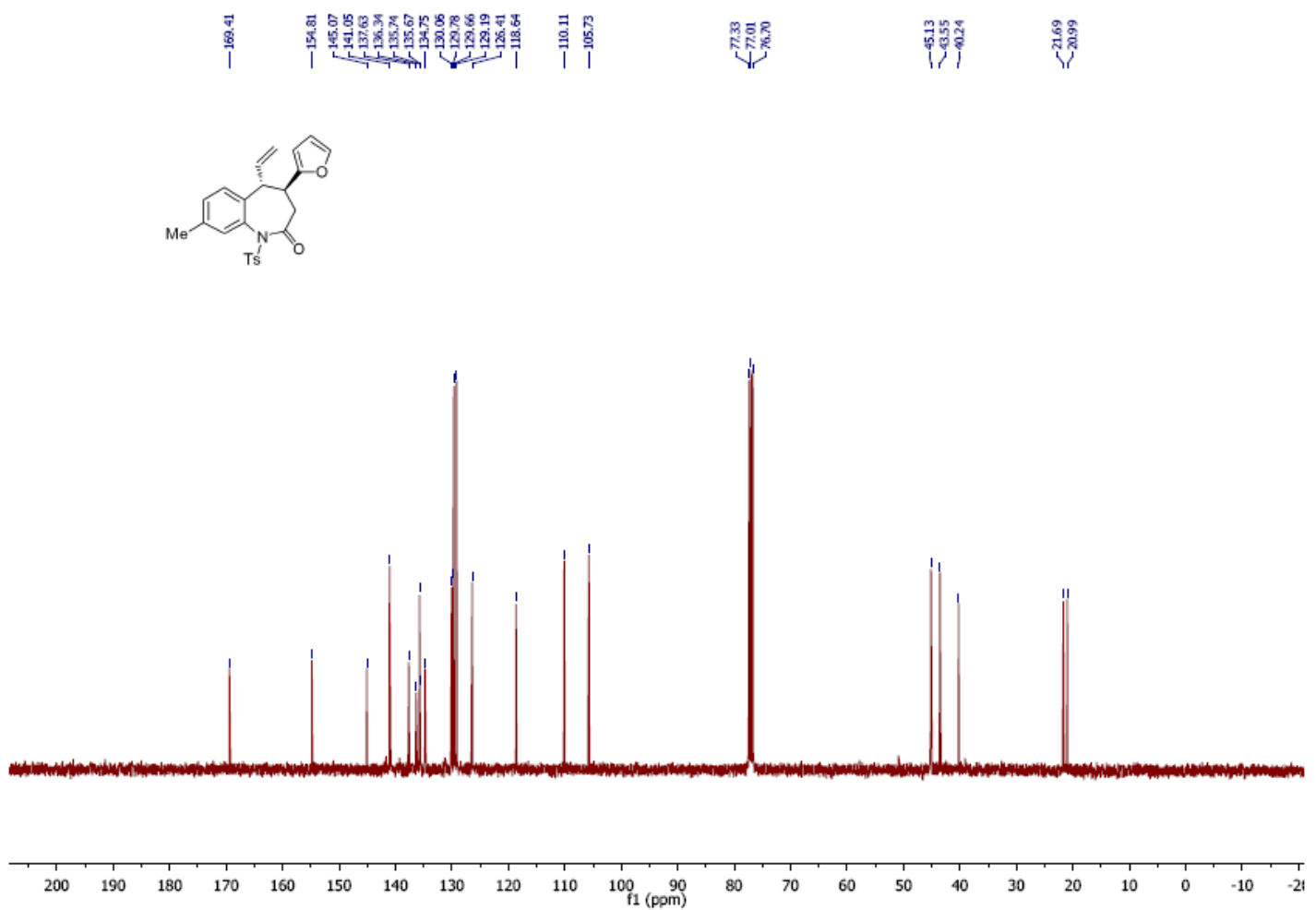
${ }^{1} \mathrm{H}$ NMR spectrum of $\mathbf{3 g k}$
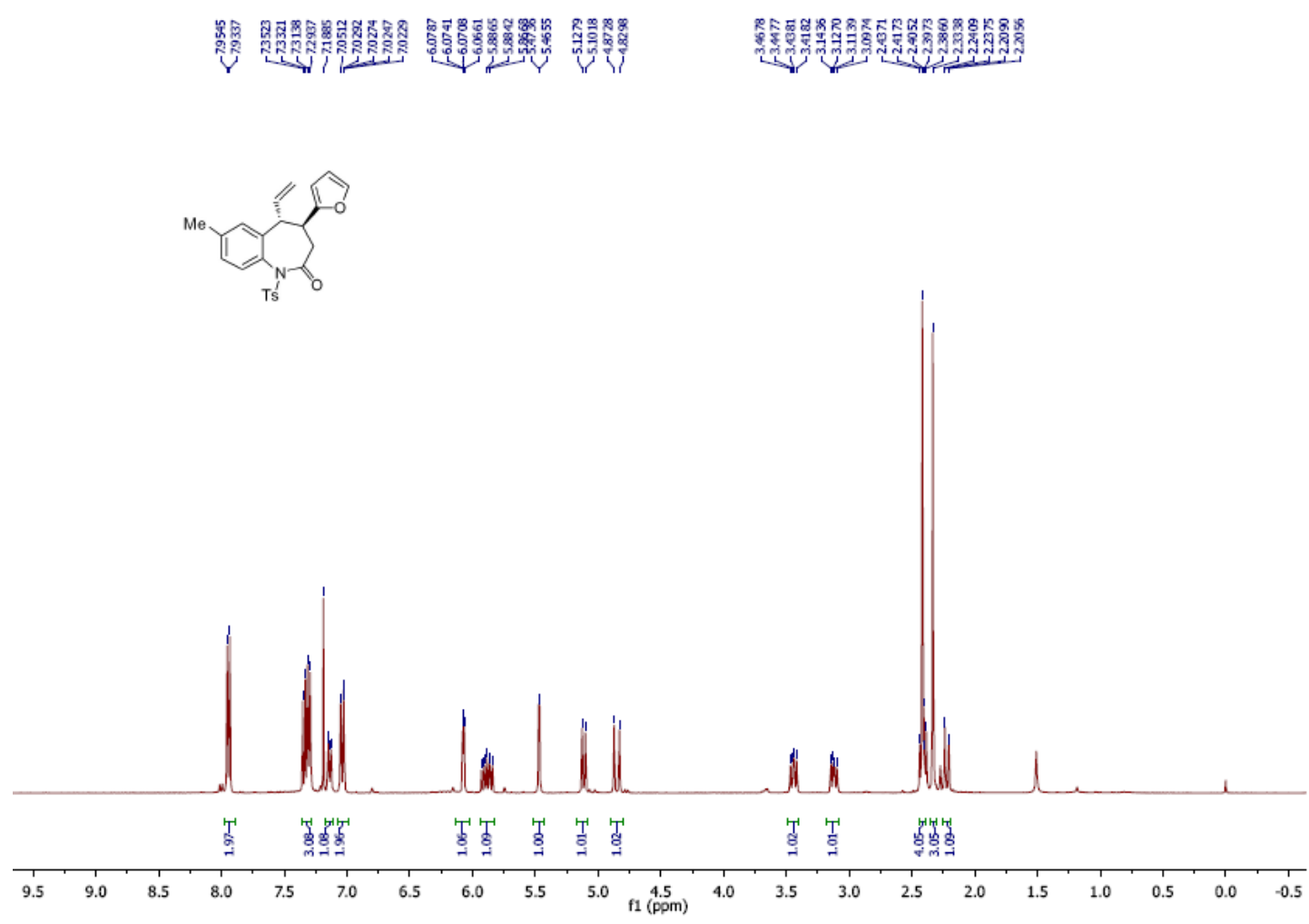

${ }^{13} \mathrm{C}$ NMR spectrum of $\mathbf{3 g k}$
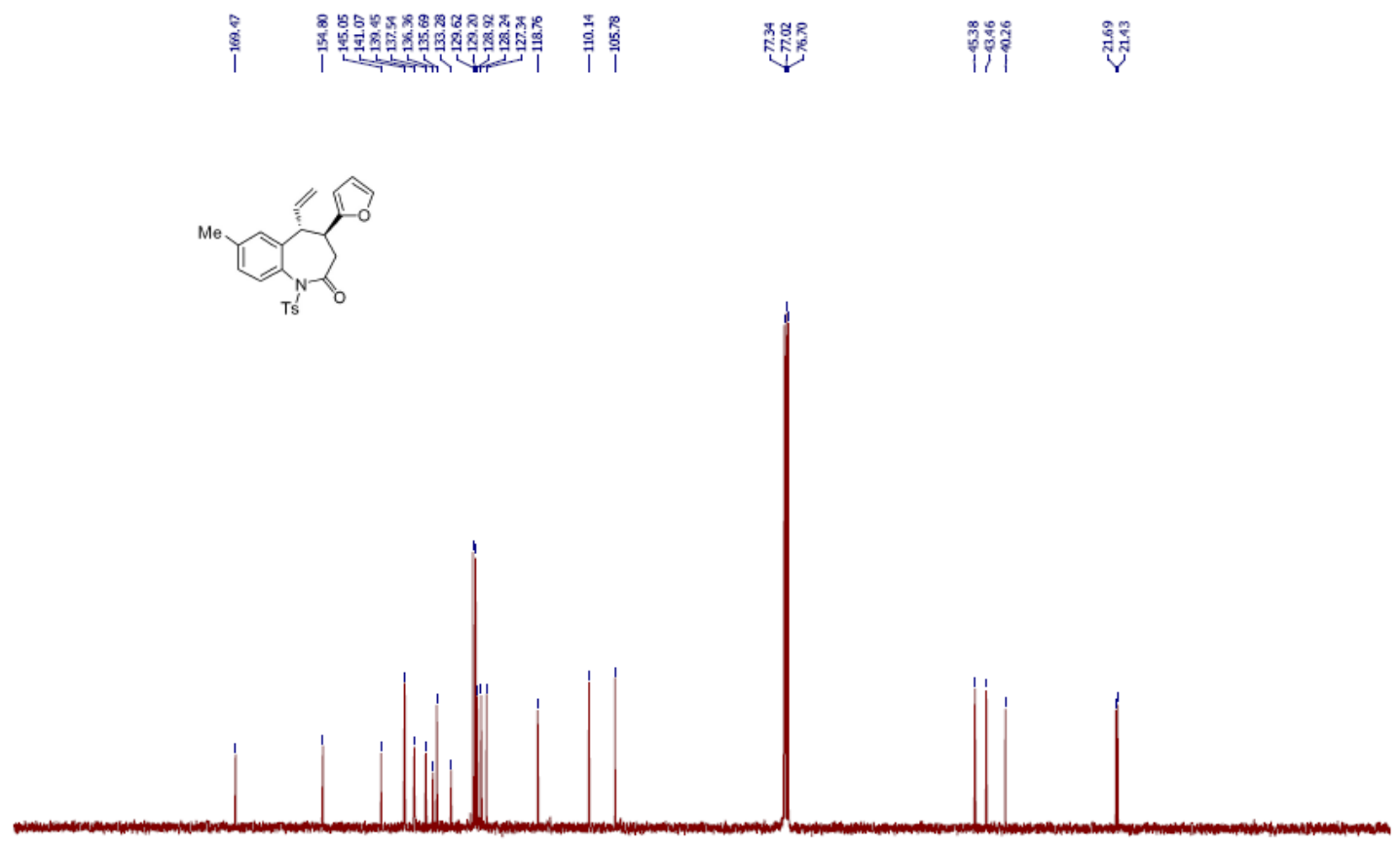

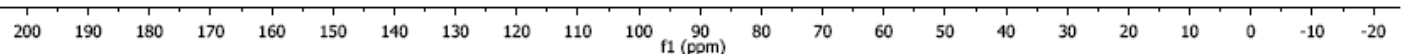


${ }^{1} \mathrm{H}$ NMR spectrum of 3ik
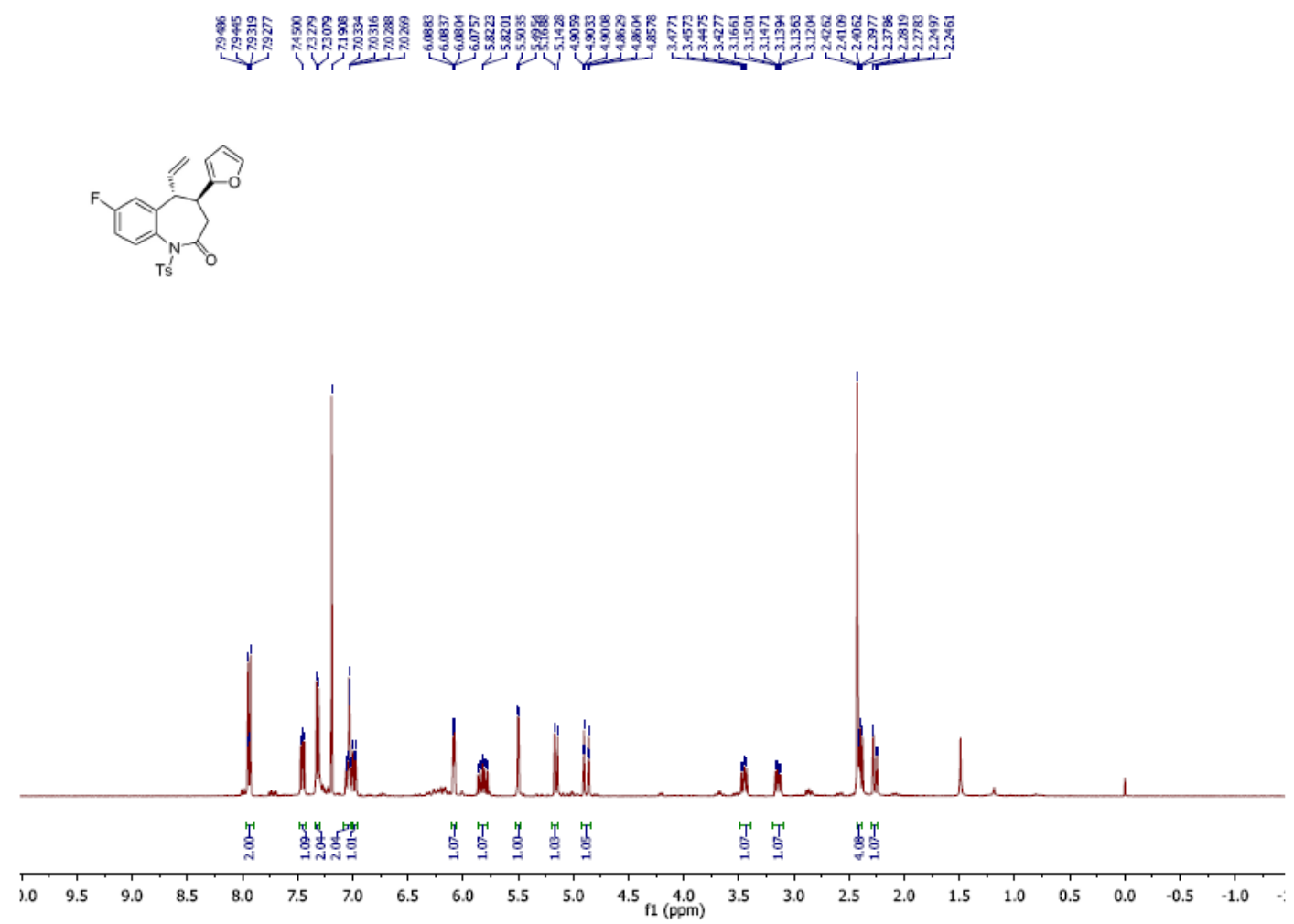

${ }^{13} \mathrm{C}$ NMR spectrum of 3ik

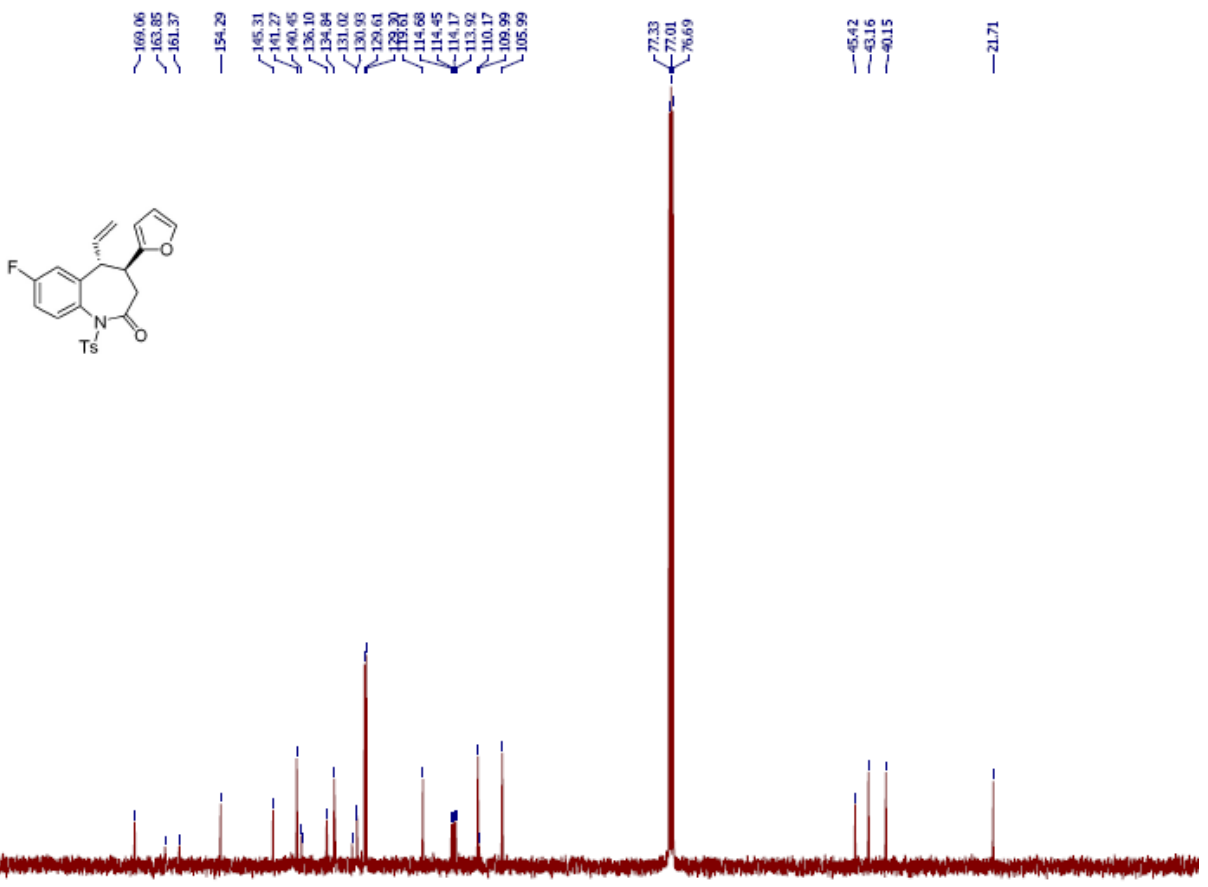

$\begin{array}{llllllllllllllllllllllllllllllllll}210 & 200 & 190 & 180 & 170 & 160 & 150 & 140 & 130 & 120 & 110 & 100 & 90 & 80 & 70 & 60 & 50 & 40 & 30 & 20 & 10 & 0 & -10\end{array}$ 
${ }^{1} \mathrm{H}$ NMR spectrum of $\mathbf{3 k} \mathbf{k}$
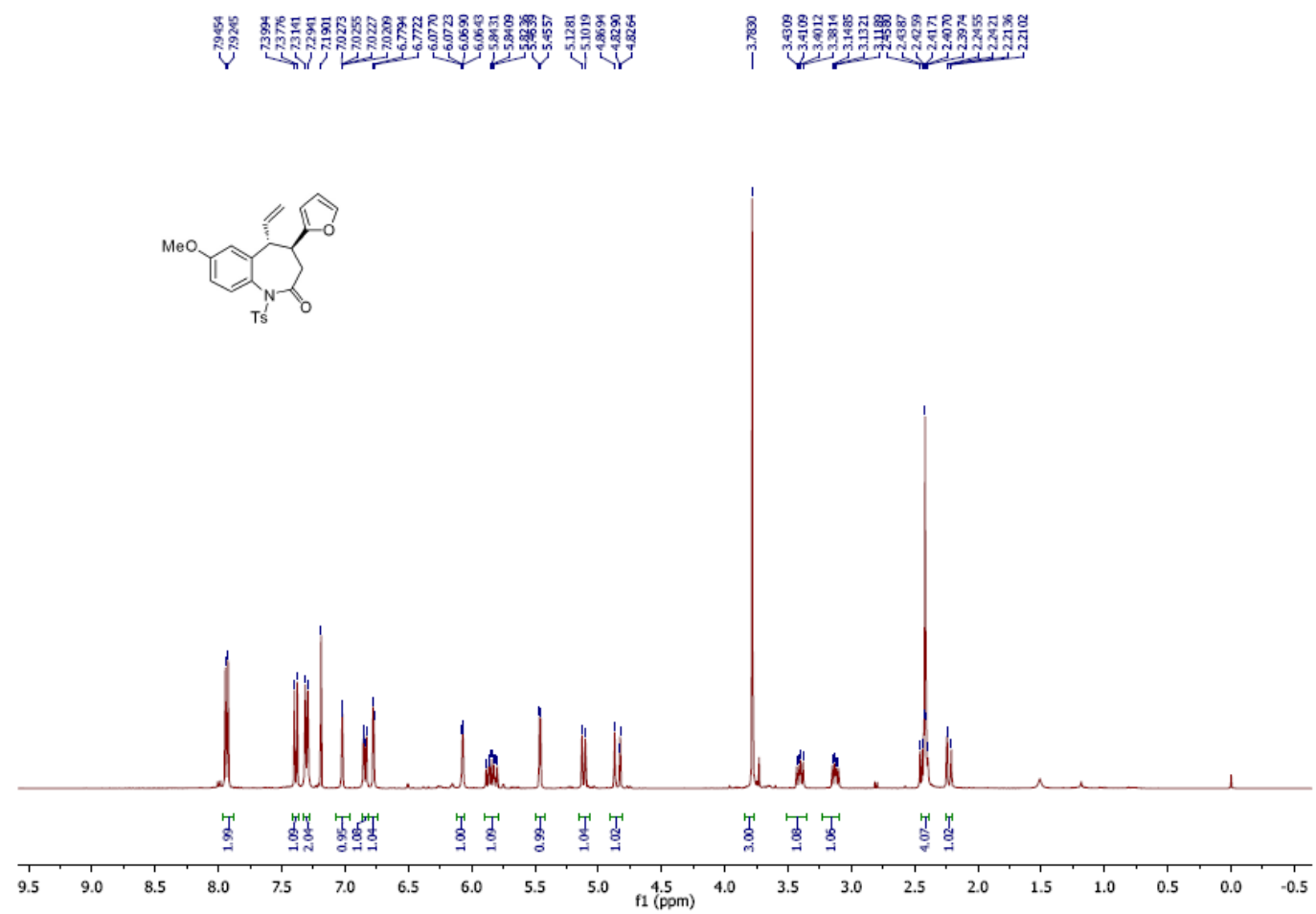

${ }^{13} \mathrm{C}$ NMR spectrum of $\mathbf{3 k} \mathbf{k}$
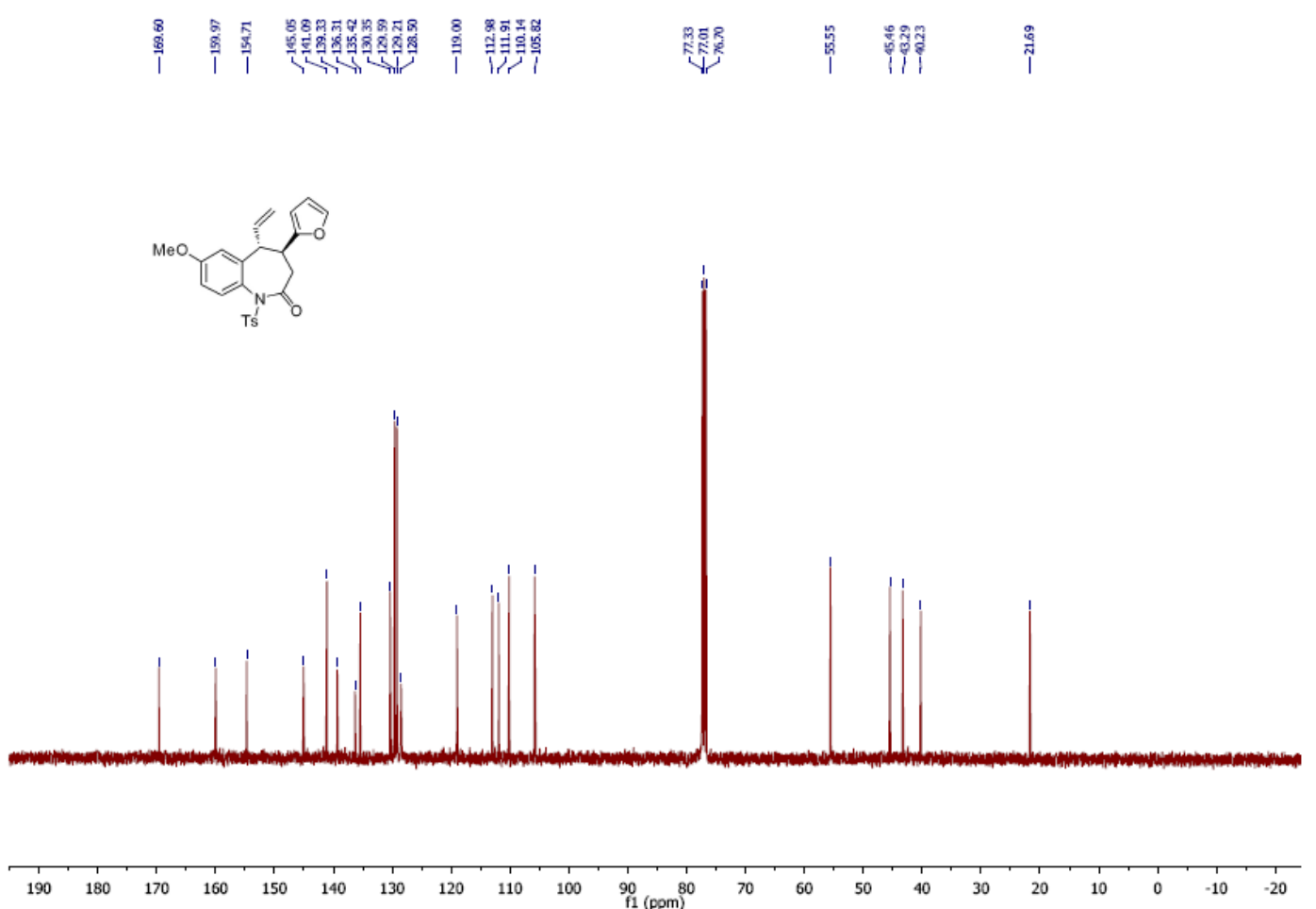
${ }^{1} \mathrm{H}$ NMR spectrum of $\mathbf{3 b c}$
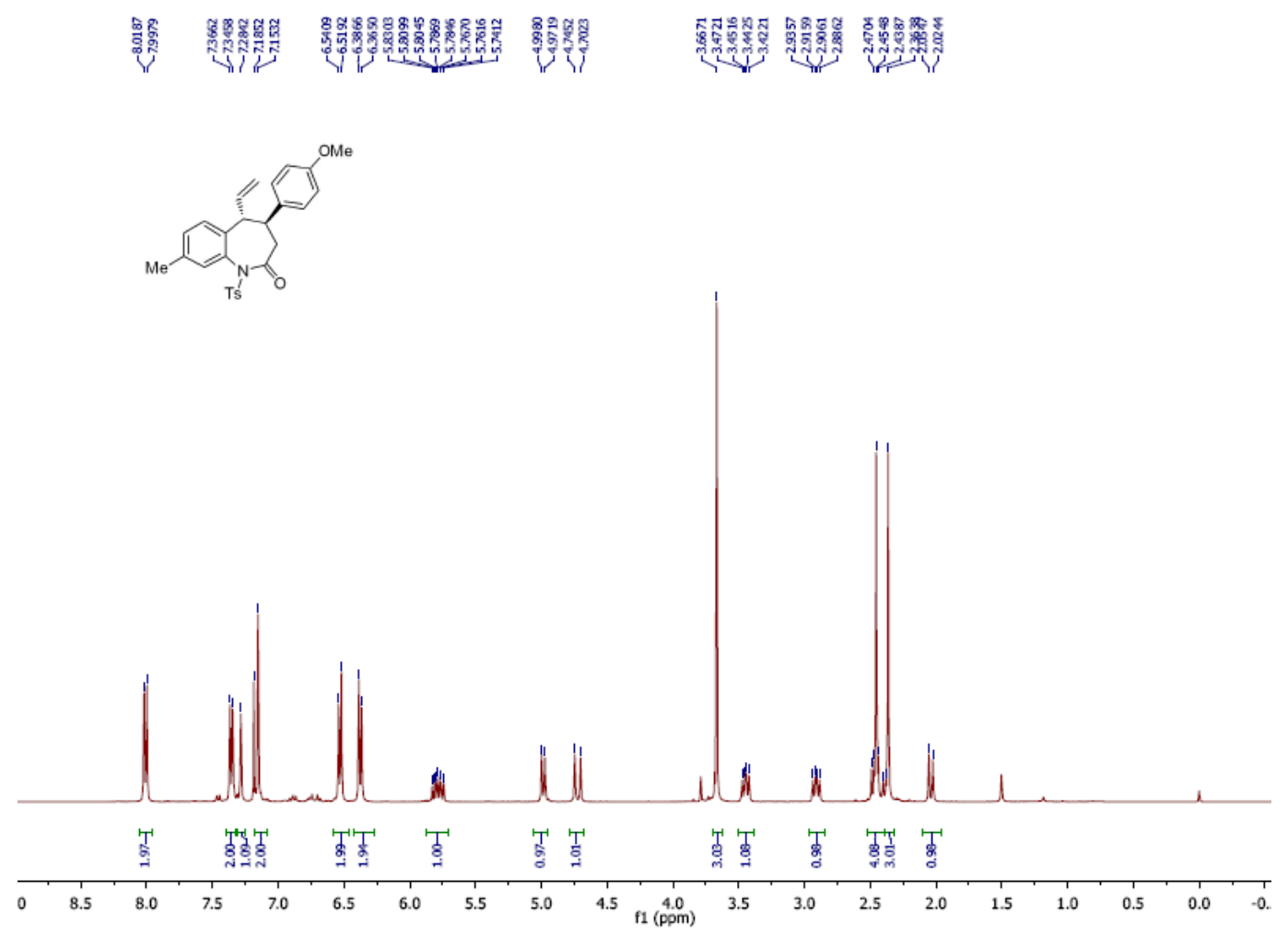

${ }^{13} \mathrm{C}$ NMR spectrum of $\mathbf{3 b c}$
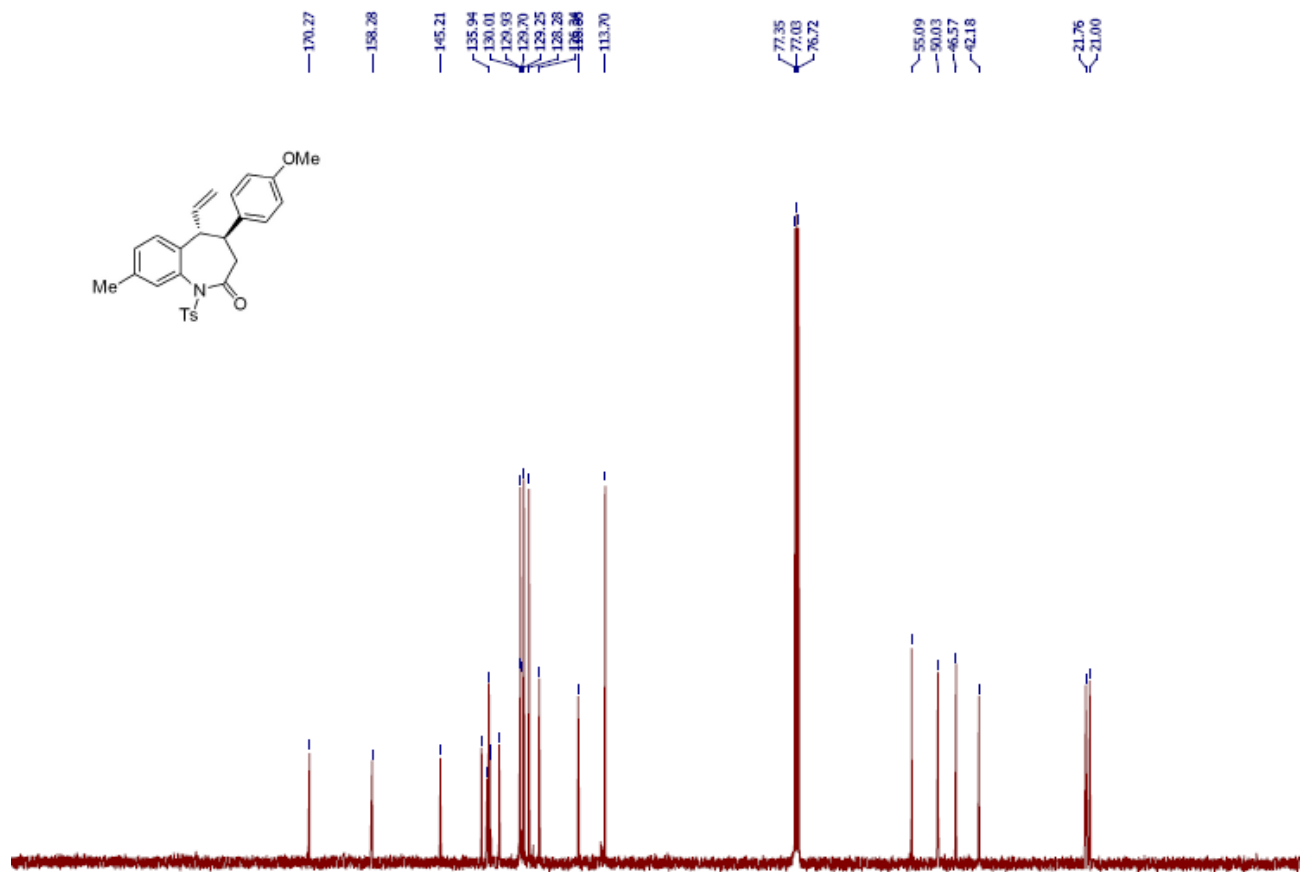

$\begin{array}{llllllllllllllllllllllllll}220 & 210 & 200 & 190 & 180 & 170 & 160 & 150 & 140 & 130 & 120 & 110 & 100 & 90 & 80 & 70 & 60 & 50 & 40 & 30 & 20 & 10 & 0 & -10 & \ddots\end{array}$ 
${ }^{1} \mathrm{H}$ NMR spectrum of $\mathbf{3 g c}$

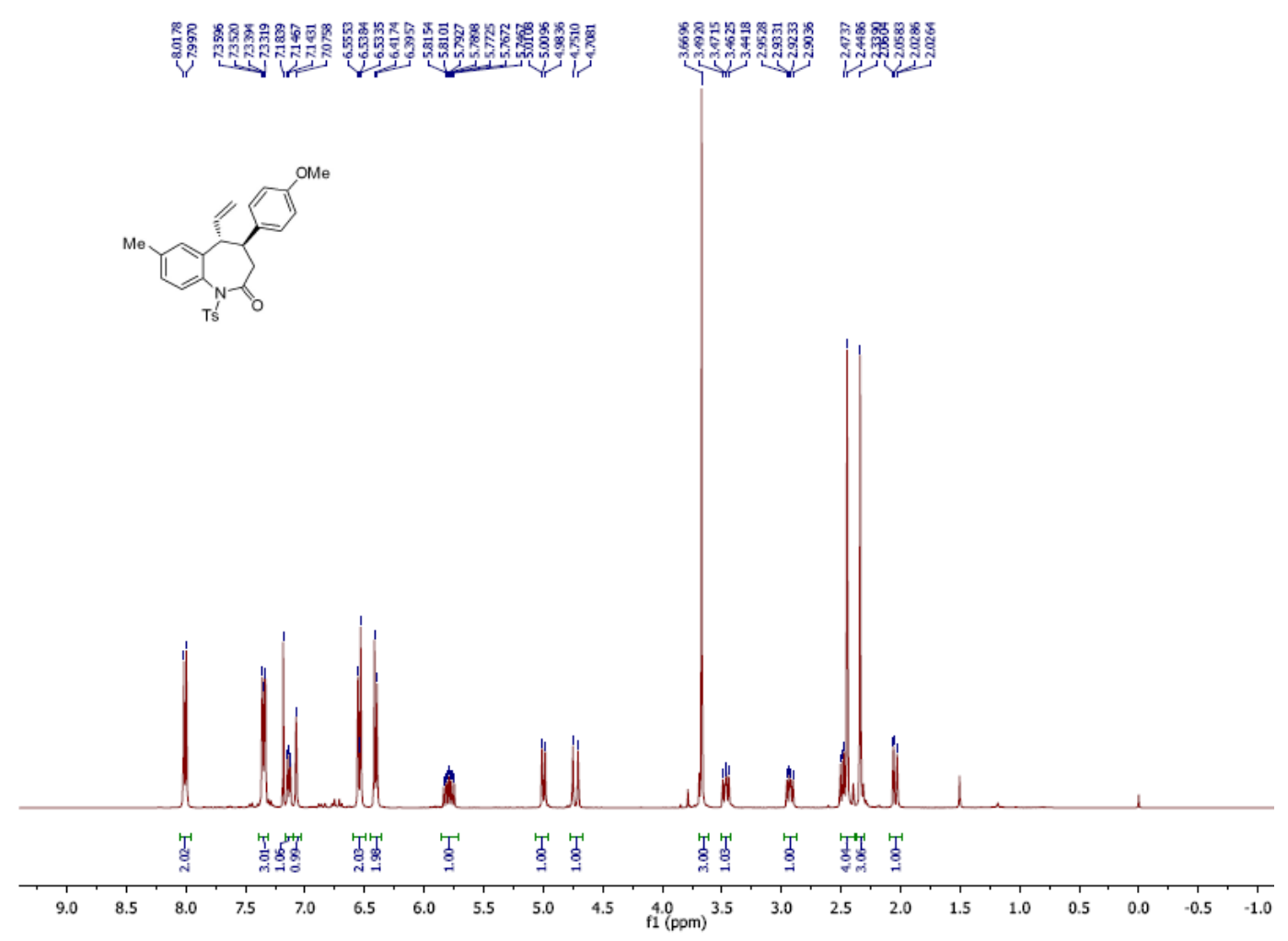

${ }^{13} \mathrm{C}$ NMR spectrum of $\mathbf{3 g c}$

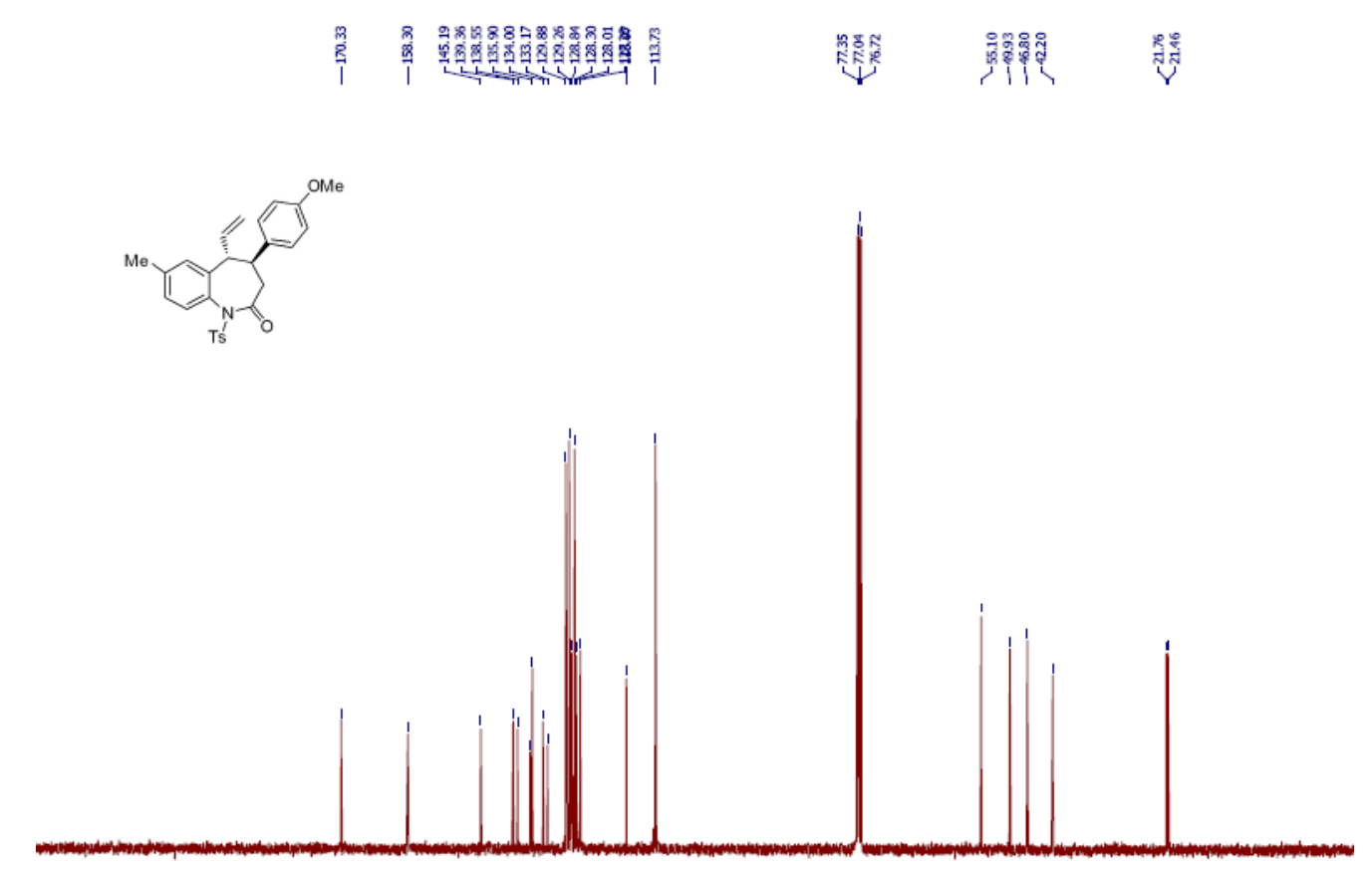

$\begin{array}{lllllllllllllllllllllllllllllllllllll}220 & 210 & 200 & 190 & 180 & 170 & 160 & 150 & 140 & 130 & 120 & 110 & 100 & 90 & 80 & 70 & 60 & 50 & 40 & 30 & 20 & 10 & 0 & -10\end{array}$ 
${ }^{1} \mathrm{H}$ NMR spectrum of $\mathbf{3 k c}$
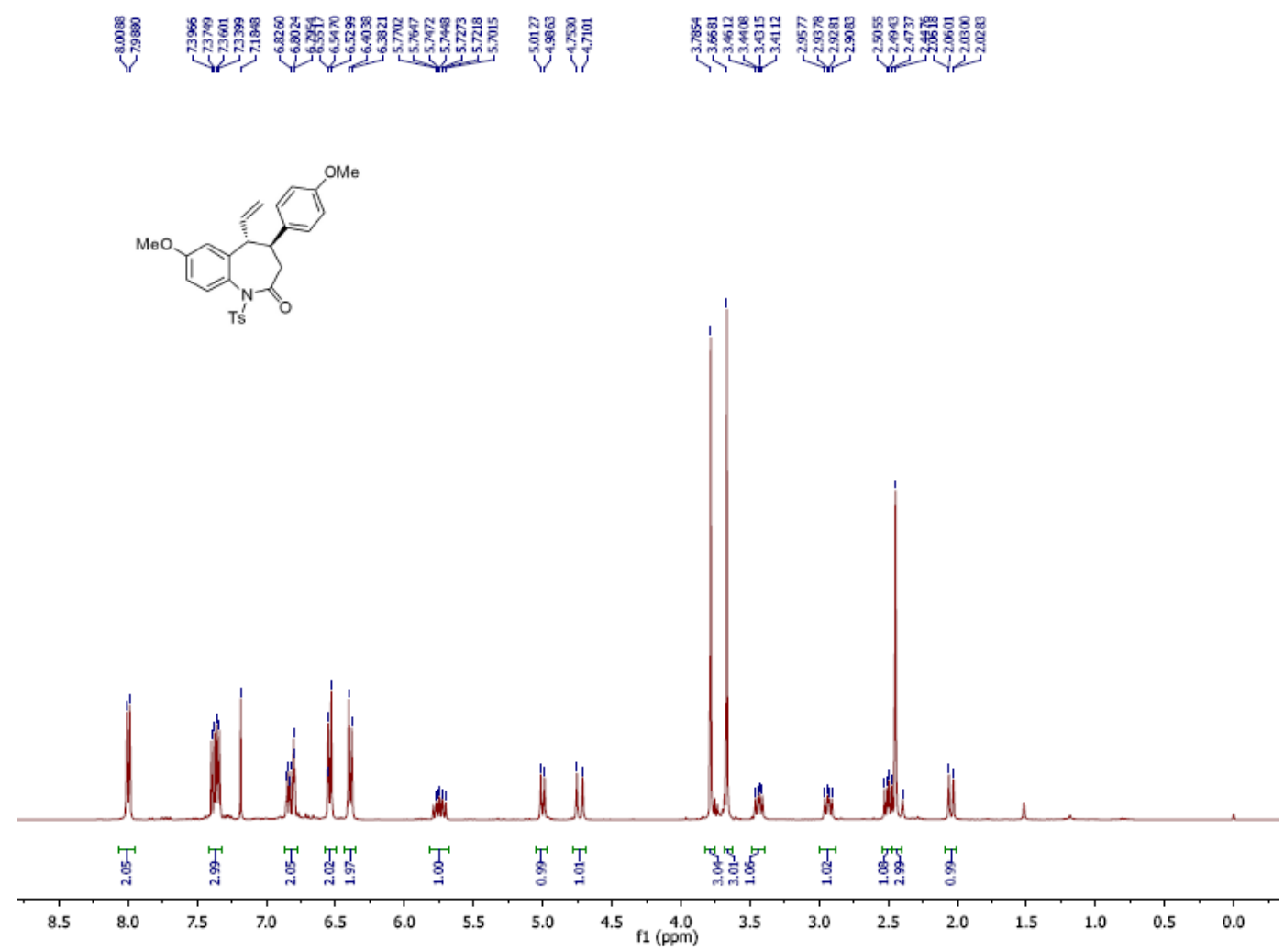

${ }^{13} \mathrm{C}$ NMR spectrum of $\mathbf{3 k c}$

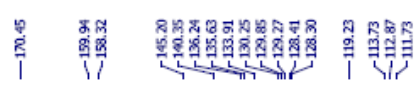
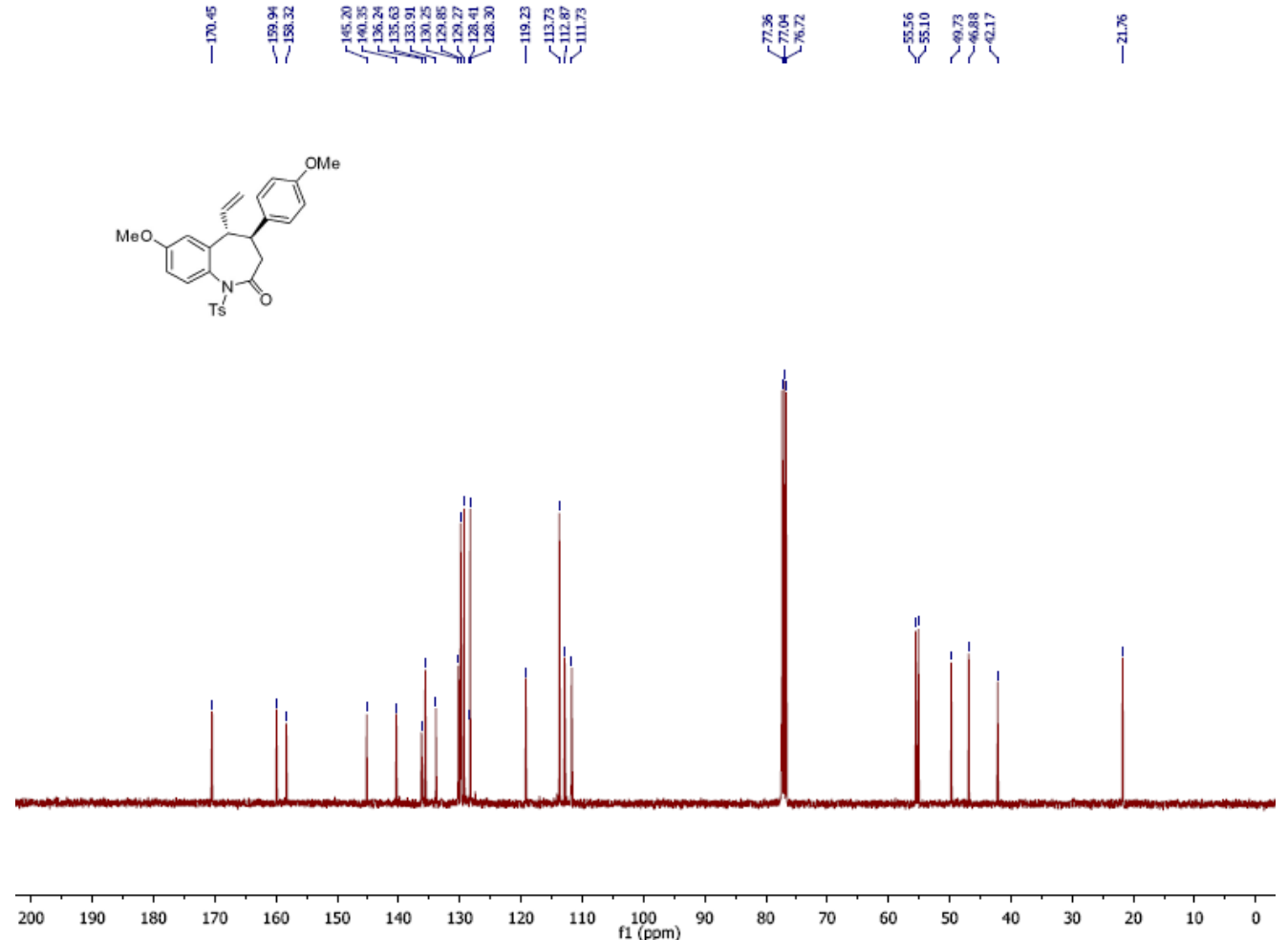
${ }^{1} \mathrm{H}$ NMR spectrum of $\mathbf{3 j \mathbf { c }}$
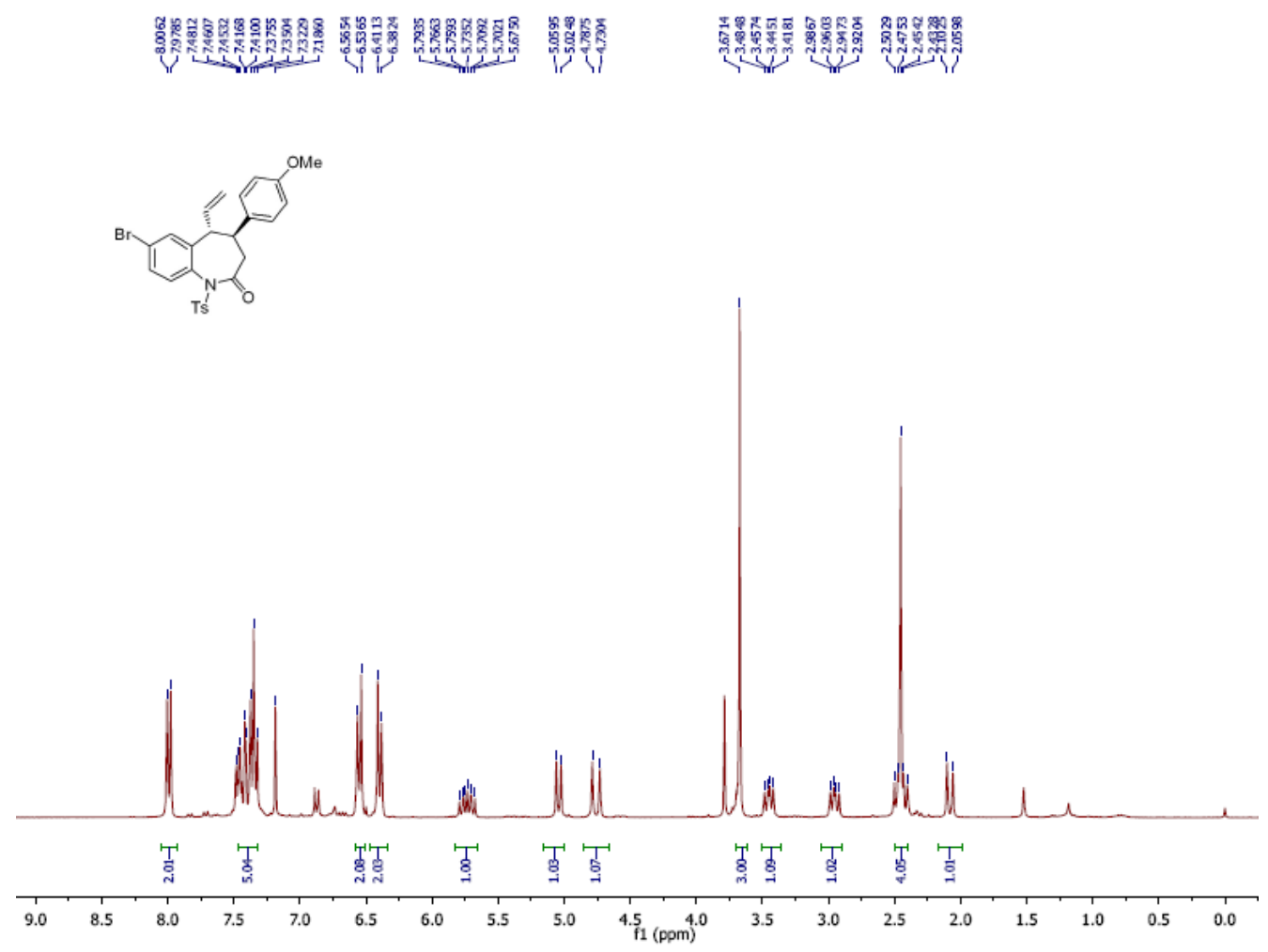

${ }^{13} \mathrm{C}$ NMR spectrum of $\mathbf{3 j c}$
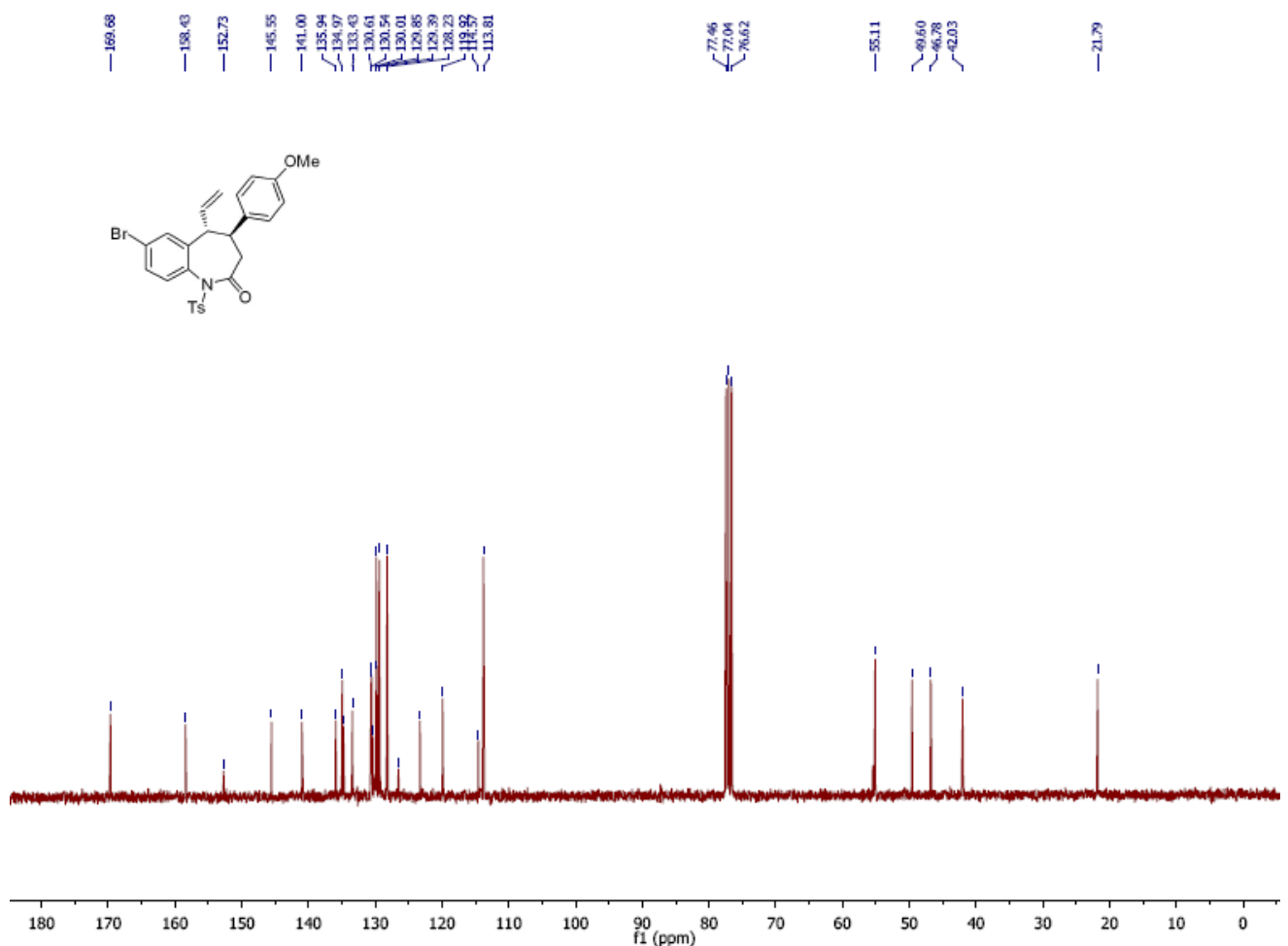
${ }^{1} \mathrm{H}$ NMR spectrum of 5

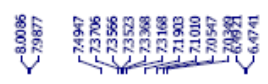

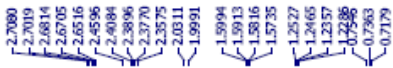
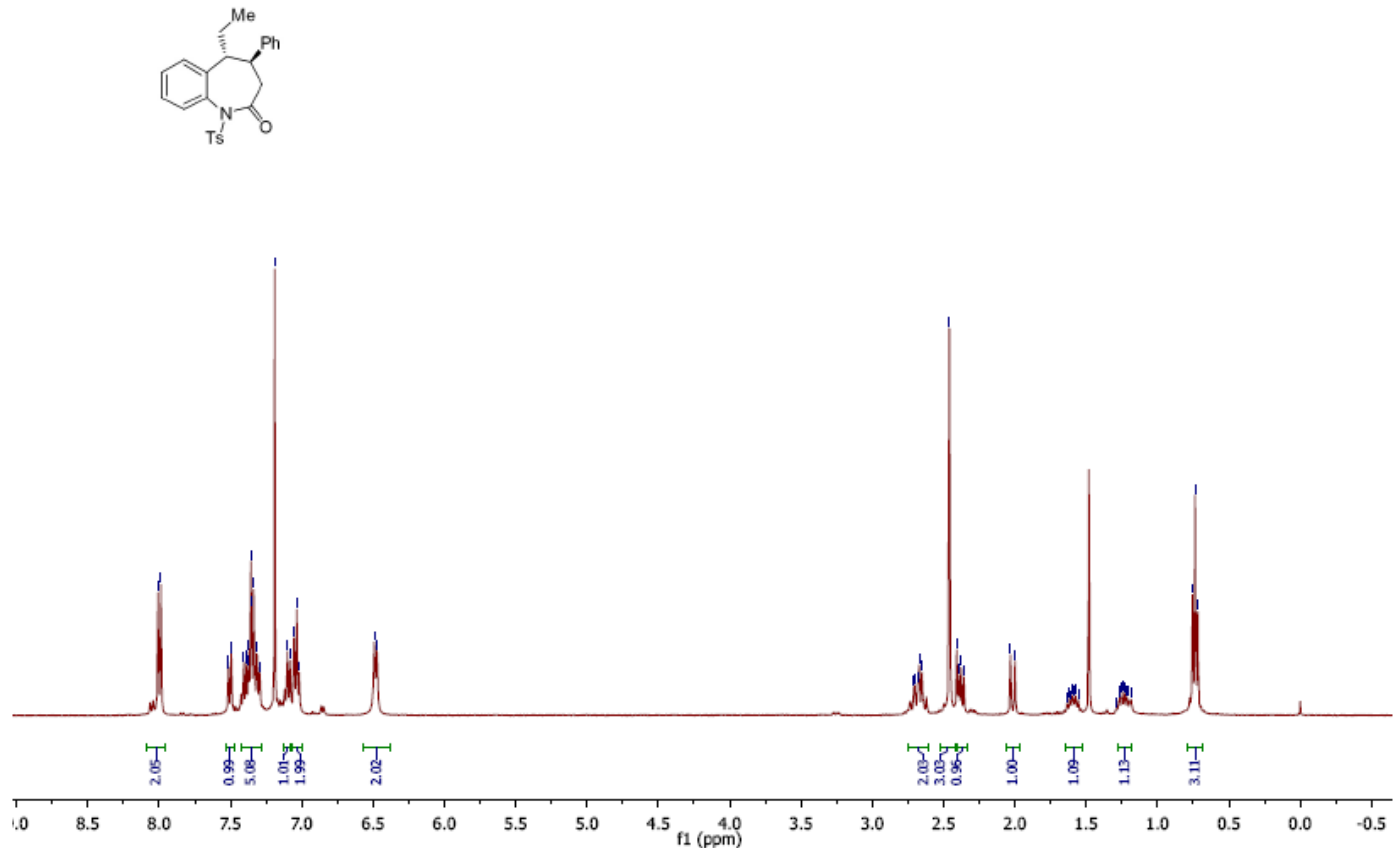

${ }^{13} \mathrm{C}$ NMR spectrum of 5
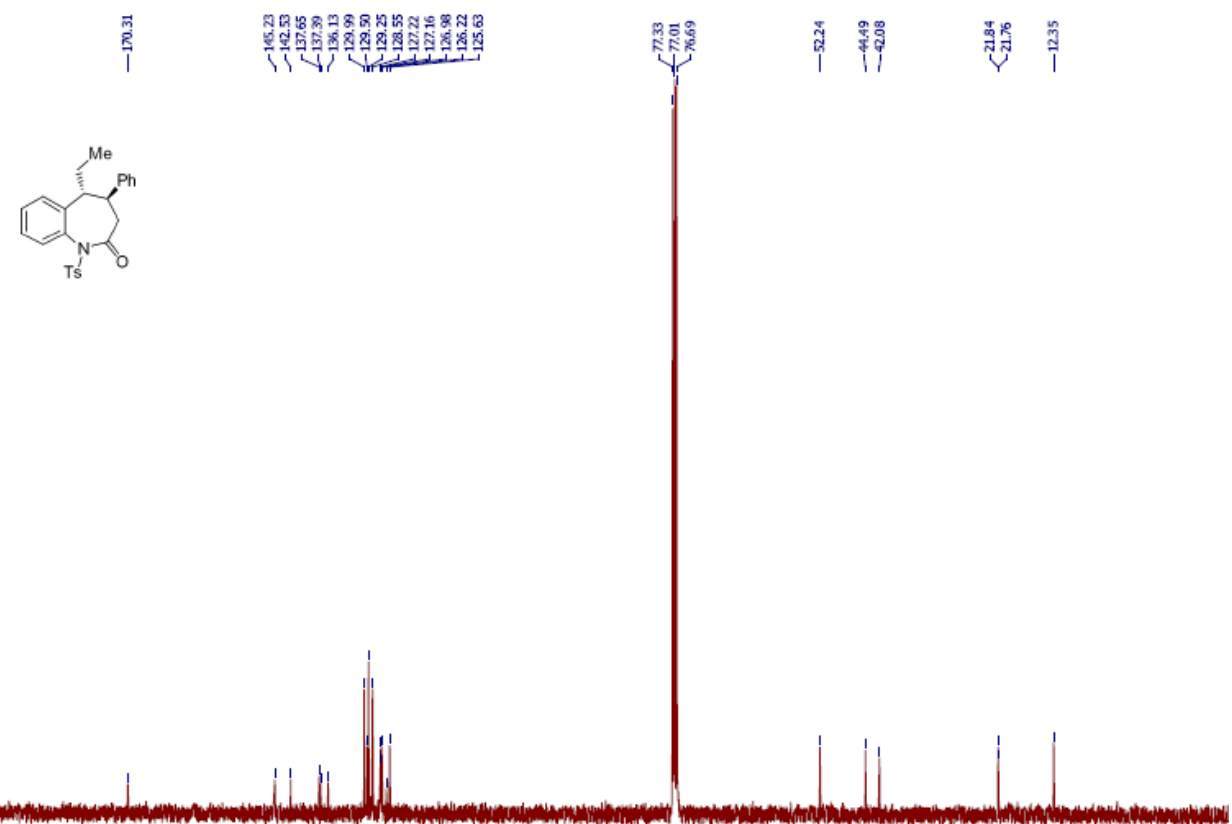

\begin{tabular}{llllllllllllllllllllllllllllllllll}
\hline 210 & 200 & 190 & 180 & 170 & 160 & 150 & 140 & 130 & 120 & 110 & 100 & 100 & 10 & 70 & 60 & 50 & 40 & 30 & 20 & 10 & 0 & -10
\end{tabular} 


\section{HPLC traces}
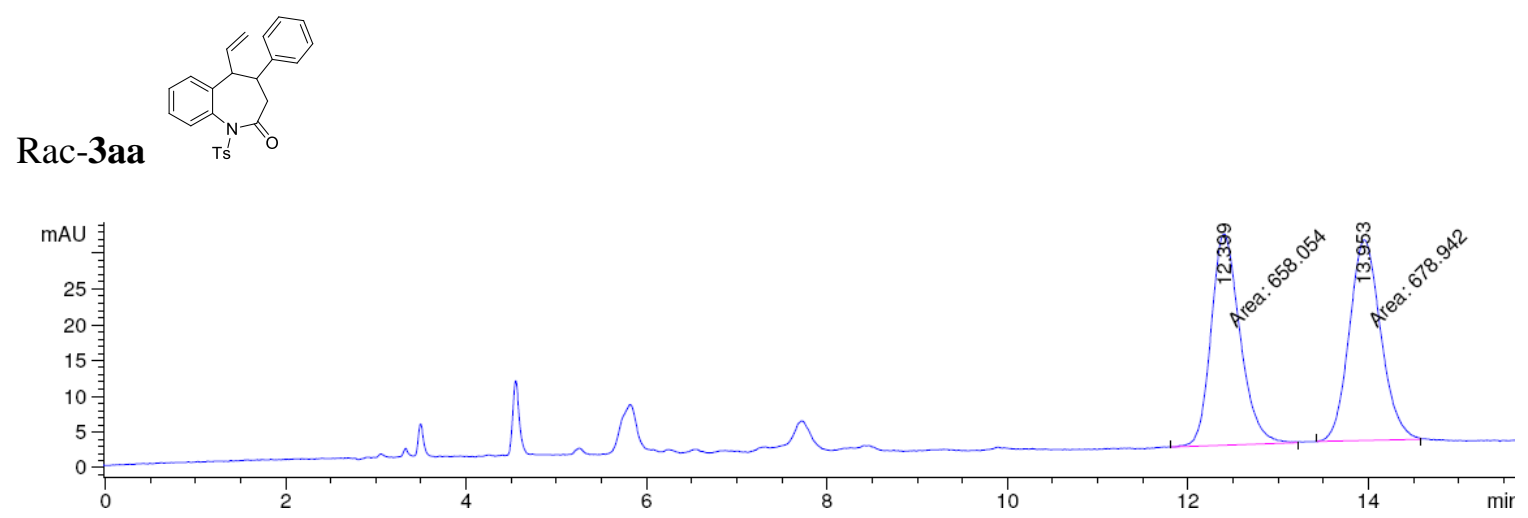

\begin{tabular}{|c|c|c|c|c|c|c|}
\hline $\begin{array}{c}\text { Peak } \\
\#\end{array}$ & $\begin{array}{c}\text { RetTime } \\
\text { [min] }\end{array}$ & Type & $\begin{array}{c}\text { Width } \\
\text { [min] }\end{array}$ & $\begin{array}{c}\text { Area } \\
{\left[\mathrm{mAU}^{*} \mathrm{~s}\right]}\end{array}$ & $\begin{array}{l}\text { Height } \\
{[\mathrm{mAU}]}\end{array}$ & $\begin{array}{c}\text { Area } \\
\%\end{array}$ \\
\hline & & & & & & \\
\hline 1 & & $\mathbb{M}$ & ? & 658.05396 & 29.52709 & 89 \\
\hline 2 & 3.953 & $\mathrm{MM}$ & 039 & 678.94171 & 28.01669 & 50.7811 \\
\hline
\end{tabular}

$\begin{array}{lll}\text { Totals : } & 1336.99567 \quad 57.54378\end{array}$
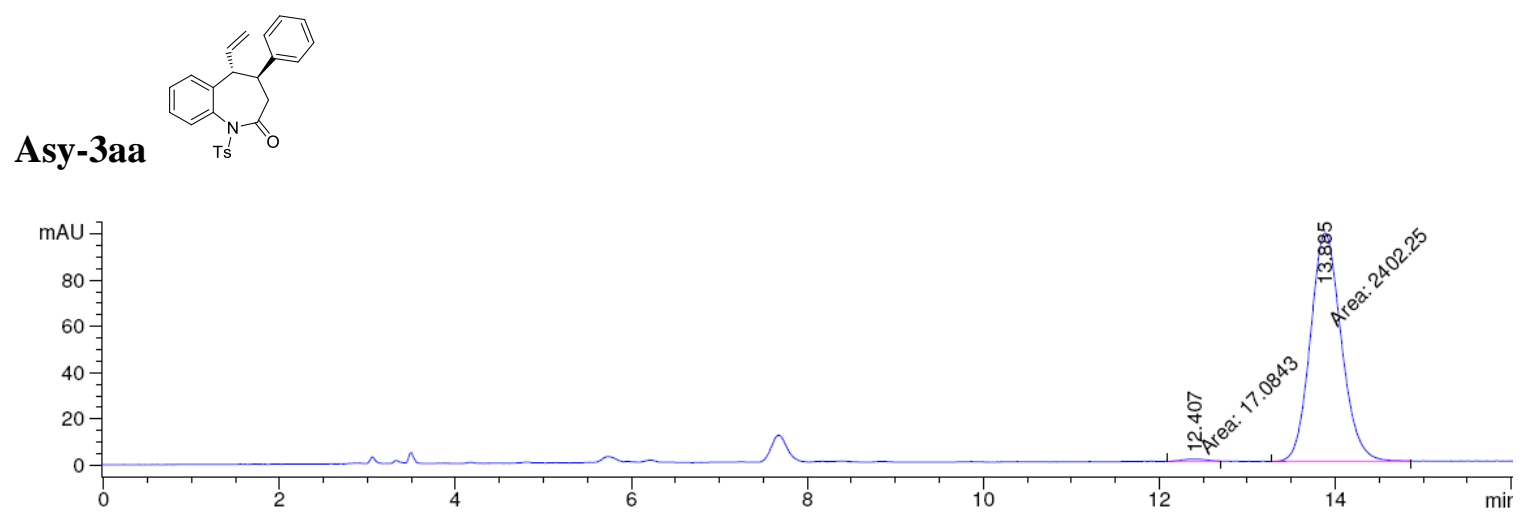

\begin{tabular}{|c|c|c|c|c|c|c|}
\hline $\begin{array}{c}\text { Peak } \\
\#\end{array}$ & $\begin{array}{c}\text { RetTime } \\
\text { [min] }\end{array}$ & Type & $\begin{array}{c}\text { Width } \\
\text { [min] }\end{array}$ & $\begin{array}{c}\text { Area } \\
{\left[\mathrm{mAU}^{*} \mathrm{~s}\right]}\end{array}$ & $\begin{array}{l}\text { Height } \\
{[\mathrm{mAU}]}\end{array}$ & $\begin{array}{c}\text { Area } \\
\%\end{array}$ \\
\hline-- & & & & -- & |--- & --- \\
\hline 1 & 12.407 & $\mathrm{MM}$ & 0 & 17.08432 & 9.092 & 7062 \\
\hline 2 & 13.885 & MM & 0.4055 & 2402.24878 & 3324 & 2938 \\
\hline
\end{tabular}

Totals : $\quad 2419.33310 \quad 99.64251$ 

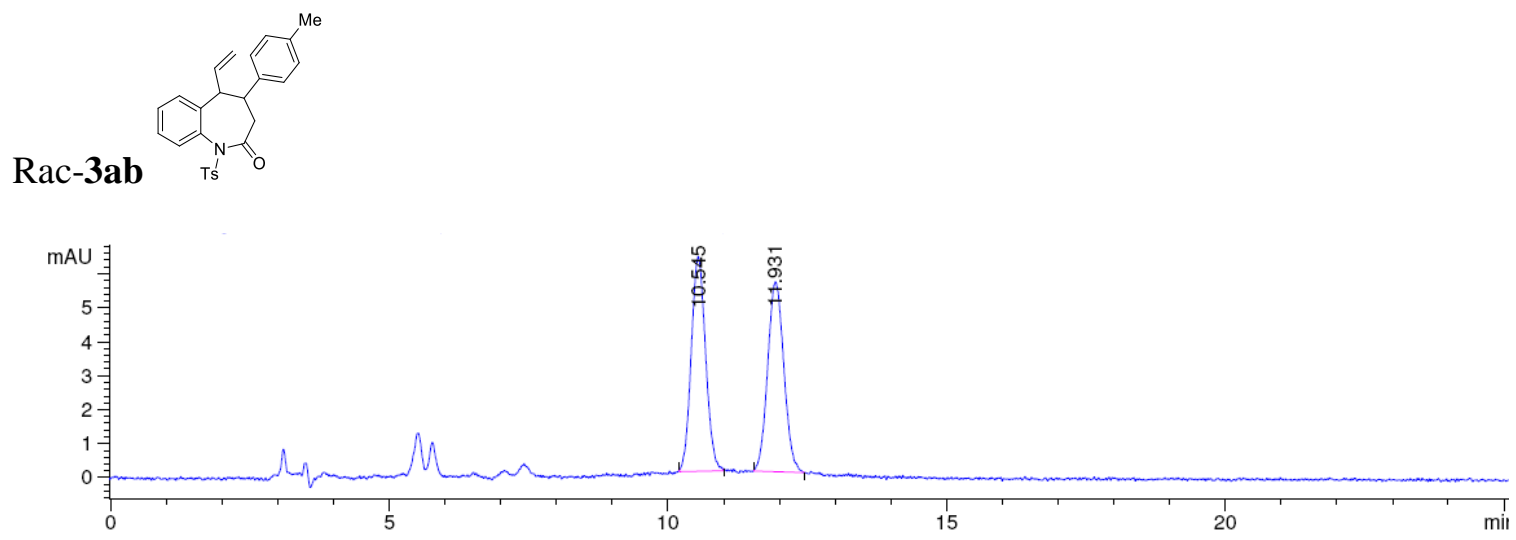

\begin{tabular}{|c|c|c|c|c|c|c|}
\hline $\begin{array}{c}\text { Peak } \\
\#\end{array}$ & $\begin{array}{c}\text { RetTime } \\
\text { [min] }\end{array}$ & Type & $\begin{array}{l}\text { Width } \\
\text { [min] }\end{array}$ & $\begin{array}{c}\text { Area } \\
{\left[\mathrm{mAU}^{*} \mathrm{~s}\right]}\end{array}$ & $\begin{array}{l}\text { Height } \\
{[\mathrm{mAU}]}\end{array}$ & $\begin{array}{c}\text { Area } \\
\%\end{array}$ \\
\hline & & & & & & \\
\hline 1 & 10 & B & 0.2223 & 113.41599 & 6.32 & 056 \\
\hline 2 & 11.931 & $\mathrm{BB}$ & 0.2495 & 112.93774 & 5.59389 & 49.8944 \\
\hline
\end{tabular}

Totals : $\quad 226.35373 \quad 11.91492$
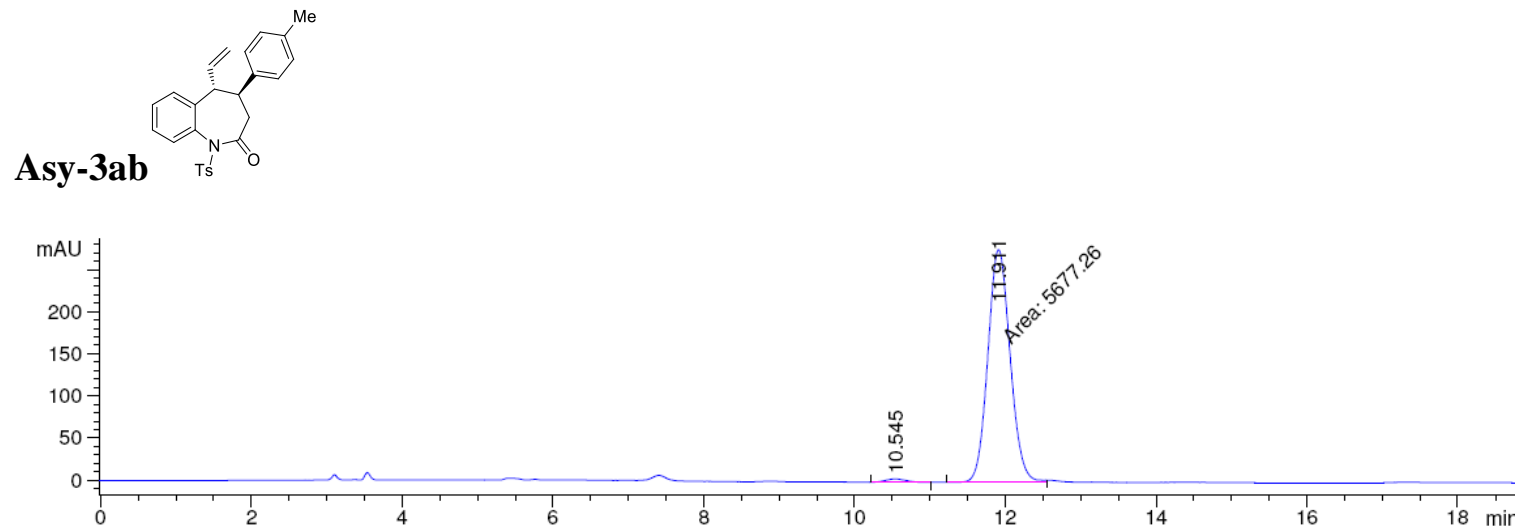

\begin{tabular}{|c|c|c|c|c|c|c|}
\hline $\begin{array}{c}\text { Peak } \\
\#\end{array}$ & $\begin{array}{c}\text { RetTime } \\
\text { [min] }\end{array}$ & Type & $\begin{array}{l}\text { Width } \\
\text { [min] }\end{array}$ & $\begin{array}{c}\text { Area } \\
{\left[\mathrm{mAU}^{*} \mathrm{~s}\right]}\end{array}$ & $\begin{array}{l}\text { Height } \\
{[\mathrm{mAU}]}\end{array}$ & $\begin{array}{c}\text { Area } \\
\%\end{array}$ \\
\hline-1 & & & & ---- & --- & -- \\
\hline 1 & 10.545 & $\mathrm{BB}$ & 208 & 72.34077 & 4.10138 & 2582 \\
\hline 2 & 11.911 & 4 & 0.3424 & 5677.25781 & 276.31989 & 98.7418 \\
\hline
\end{tabular}

Totals :

$5749.59858 \quad 280.42126$ 

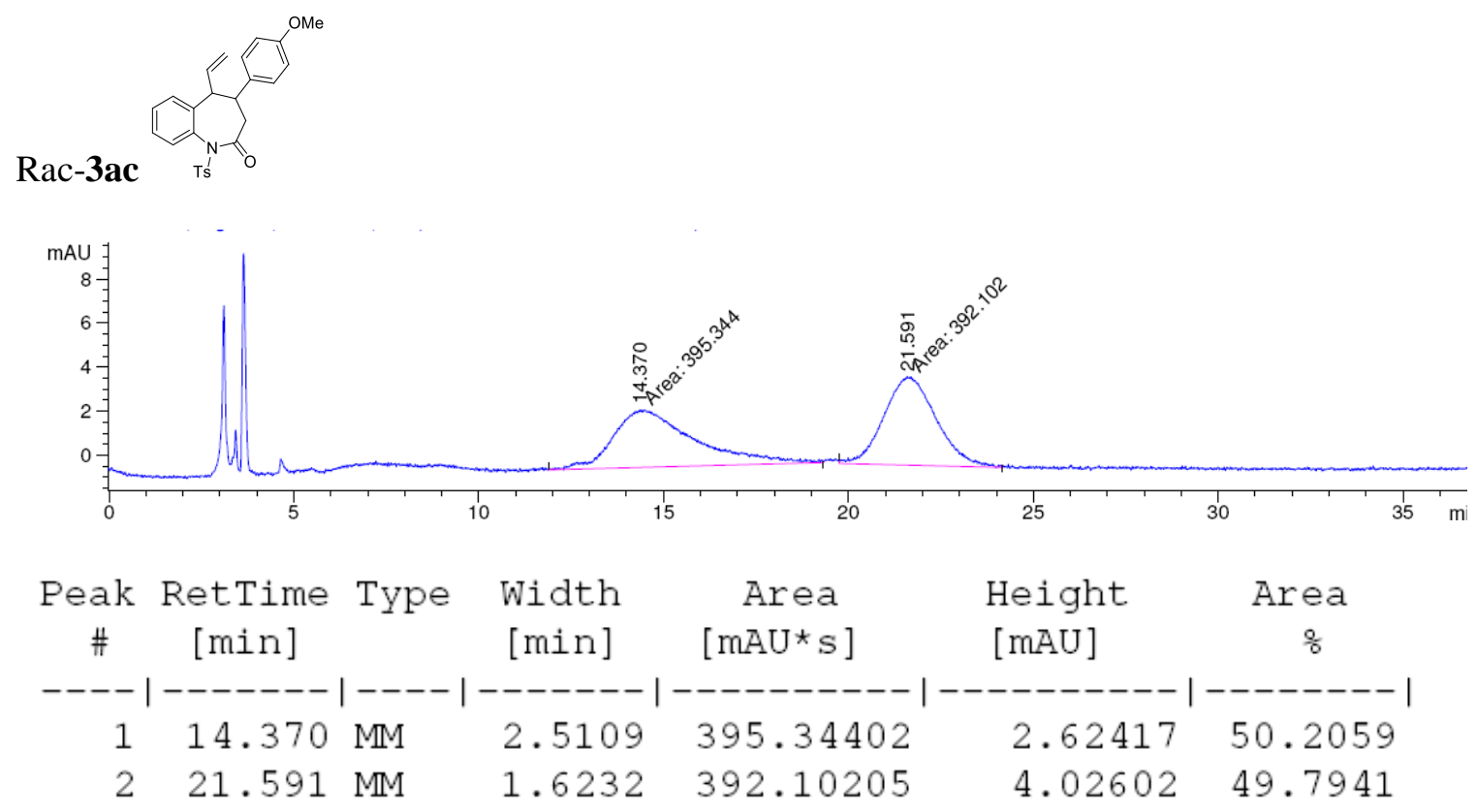

Totals :

$787.44608 \quad 6.65019$
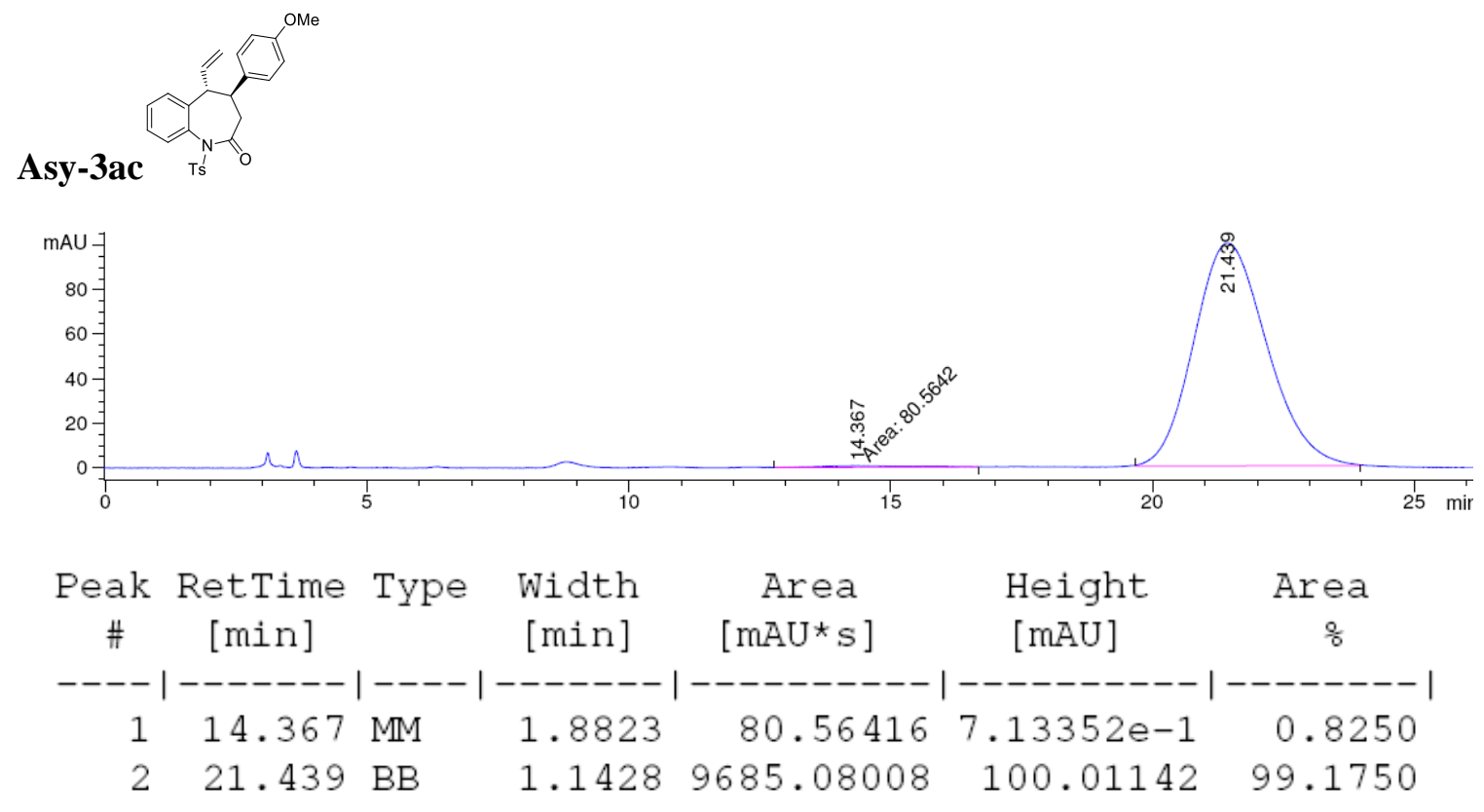

Totals :

$9765.64424 \quad 100.72477$ 


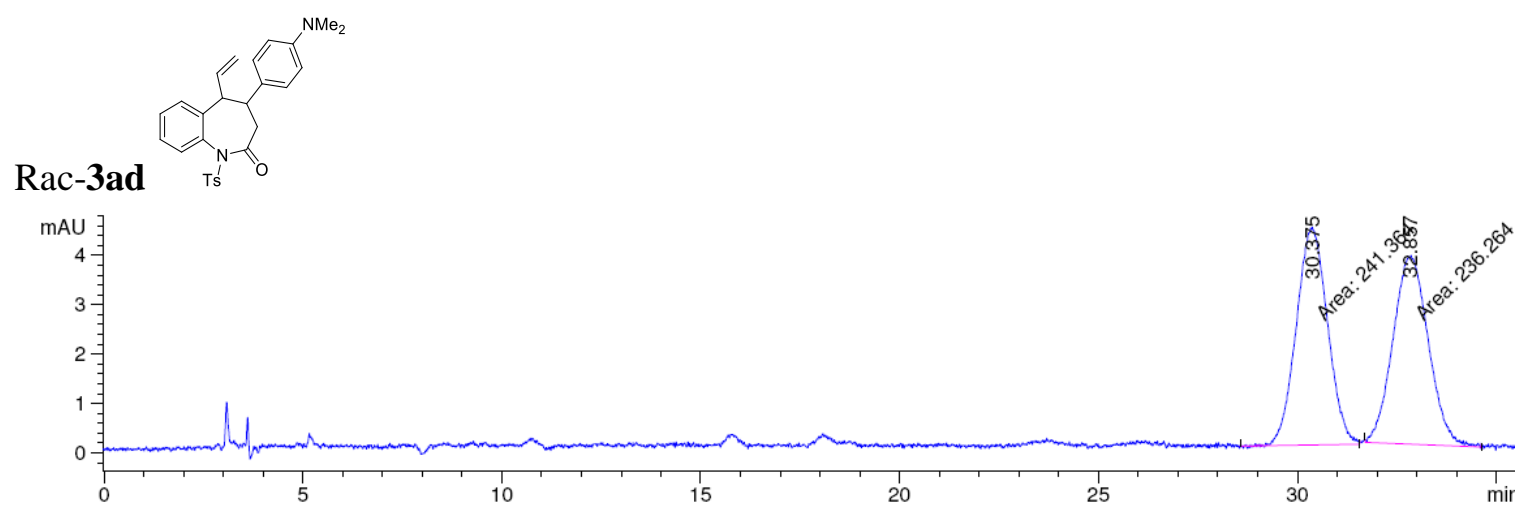

\begin{tabular}{|c|c|c|c|c|c|c|}
\hline $\begin{array}{c}\text { Peak } \\
\#\end{array}$ & $\begin{array}{c}\text { RetTime } \\
\text { [min] }\end{array}$ & Type & $\begin{array}{l}\text { Width } \\
\text { [min] }\end{array}$ & $\begin{array}{c}\text { Area } \\
{\left[\mathrm{mAU}^{\star} \mathrm{s}\right]}\end{array}$ & $\begin{array}{l}\text { Height } \\
{[\mathrm{mAU}]}\end{array}$ & $\begin{array}{c}\text { Area } \\
\%\end{array}$ \\
\hline & & & & ---------- & -------- & ------- \\
\hline 1 & 30.375 & MM & 0.9105 & 241.36411 & 4.41821 & 50.5340 \\
\hline 2 & 32.857 & MM & 1.0283 & 236.26352 & 3.82928 & 49.4660 \\
\hline Total & $\mathrm{S}:$ & & & 477.62762 & 8.24749 & \\
\hline
\end{tabular}
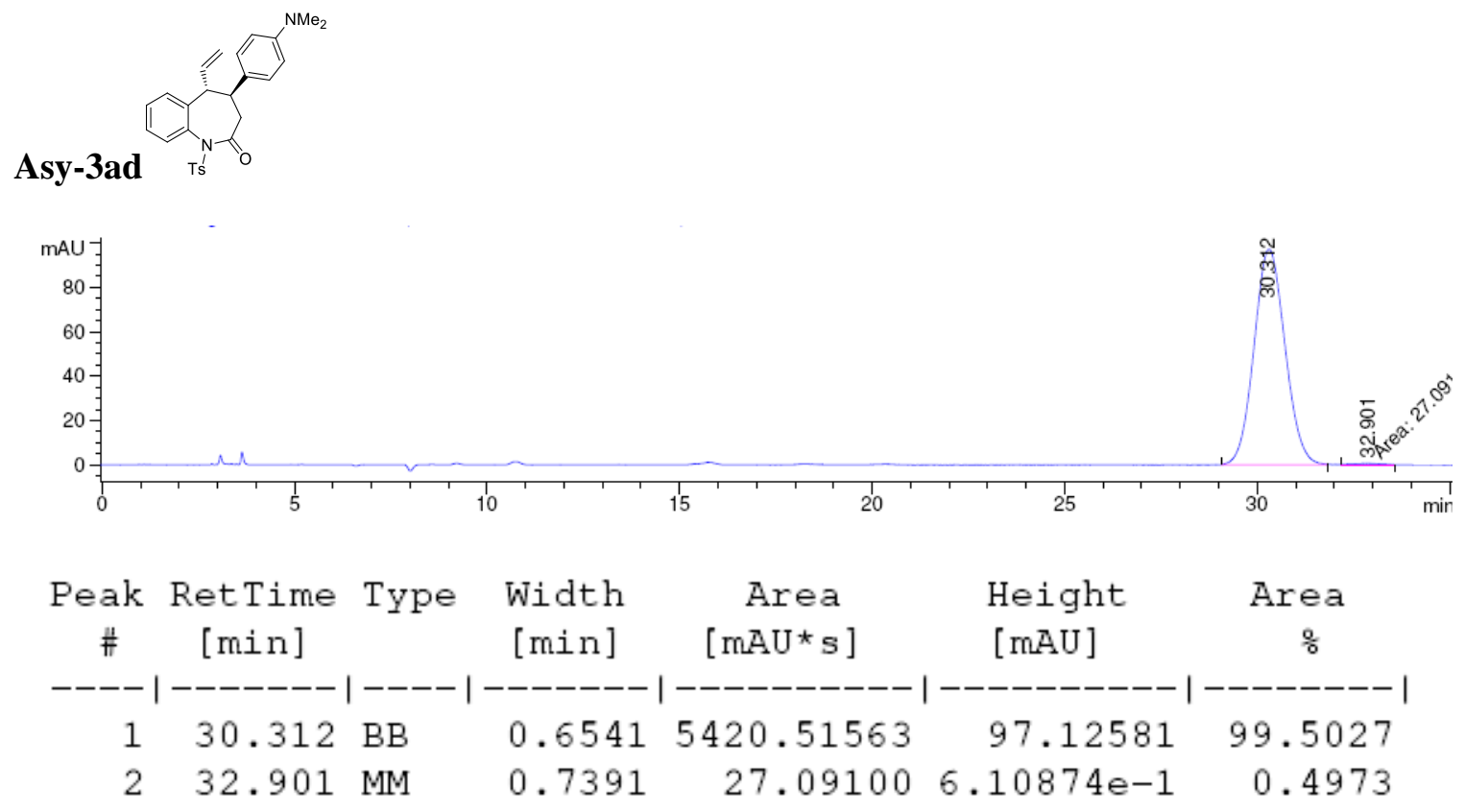

Totals :

$5447.60663 \quad 97.73668$ 

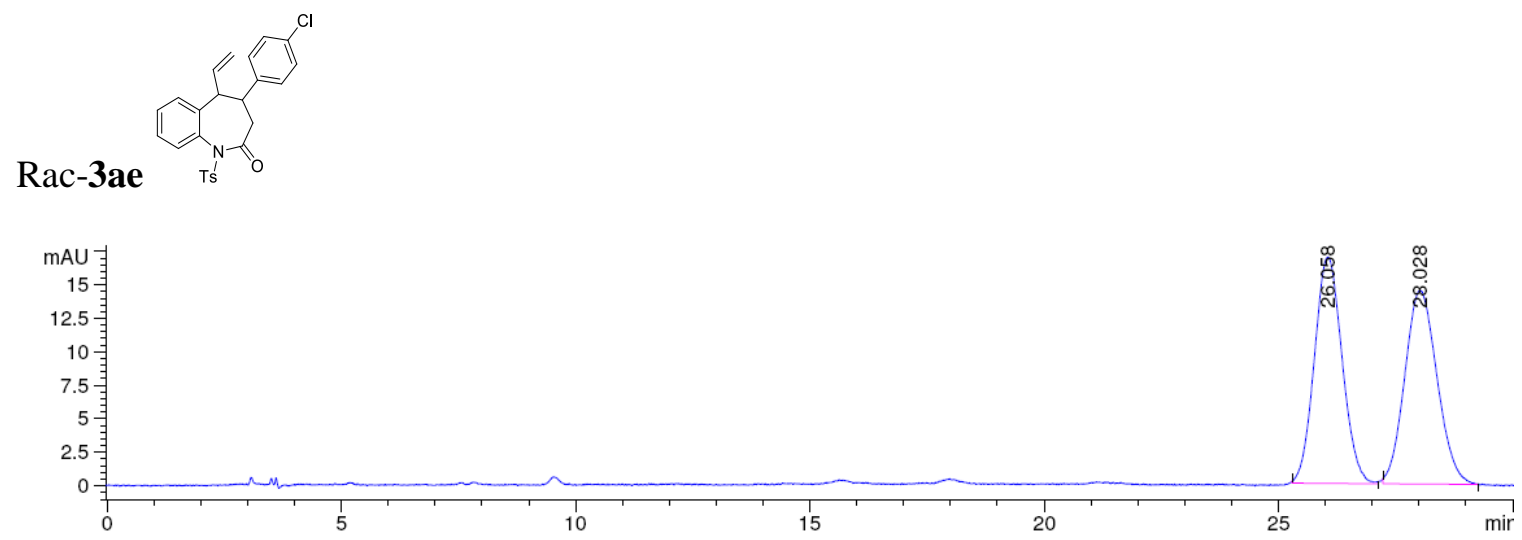

\begin{tabular}{|c|c|c|c|c|c|c|}
\hline $\begin{array}{c}\text { Peak } \\
\#\end{array}$ & $\begin{array}{c}\text { RetTime } \\
\text { [min] }\end{array}$ & Type & $\begin{array}{l}\text { Width } \\
\text { [min] }\end{array}$ & $\begin{array}{c}\text { Area } \\
{\left[\mathrm{mAU}^{\star} \mathrm{s}\right]}\end{array}$ & $\begin{array}{l}\text { Height } \\
{[\mathrm{mAU}]}\end{array}$ & $\begin{array}{c}\text { Area } \\
\%\end{array}$ \\
\hline & & & & -------- & & \\
\hline 1 & 26.05 & $B B$ & 0187 & 696.80127 & 83 & 47 \\
\hline 2 & 28.028 & $\mathrm{BB}$ & .5664 & 693.33508 & 14.43531 & 49.8753 \\
\hline
\end{tabular}

Totals : $\quad 1390.13635 \quad 31.33515$
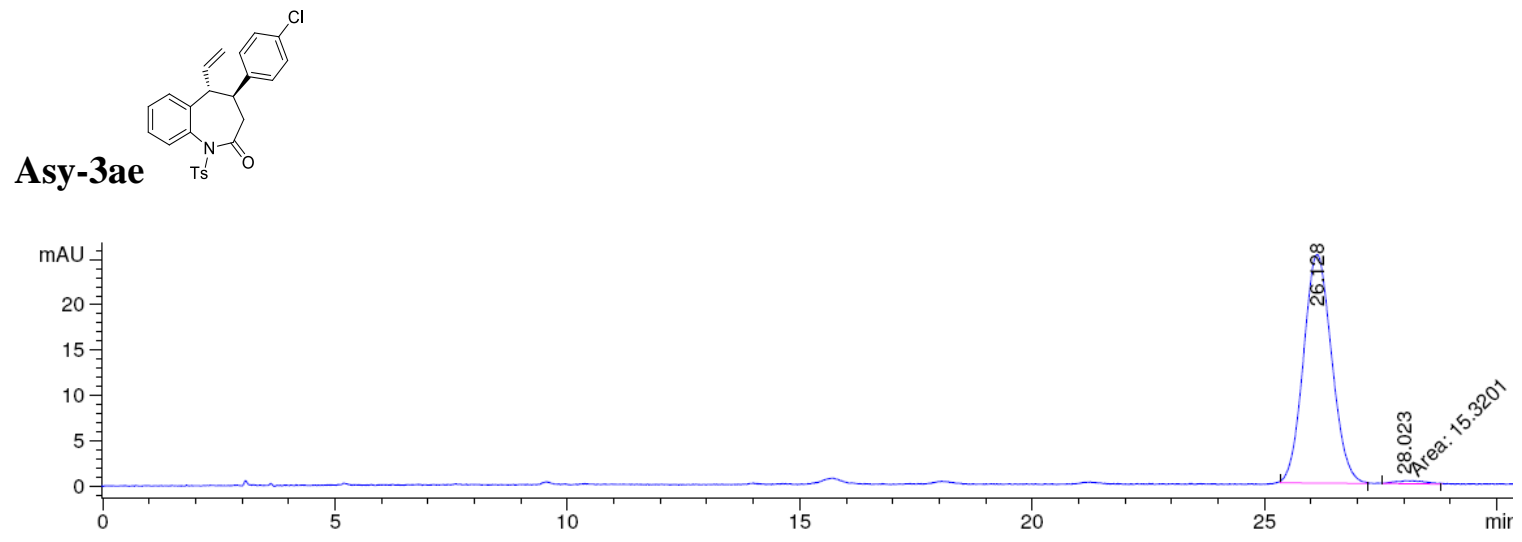

\begin{tabular}{|c|c|c|c|c|c|c|}
\hline $\begin{array}{c}\text { Peak } \\
\quad \#\end{array}$ & $\begin{array}{c}\text { RetTime } \\
\text { [min] }\end{array}$ & Type & $\begin{array}{l}\text { Width } \\
\text { [min] }\end{array}$ & $\begin{array}{c}\text { Area } \\
{\left[\mathrm{mAU}^{\star} \mathrm{s}\right]}\end{array}$ & $\begin{array}{l}\text { Height } \\
{[\mathrm{mAU}]}\end{array}$ & $\begin{array}{c}\text { Area } \\
\%\end{array}$ \\
\hline & & & & $-----------\mid$ & 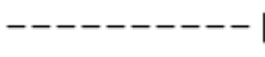 & \\
\hline 1 & 128 & B & & 1064.09924 & 435 & $30^{\circ}$ \\
\hline 2 & 8.023 & MM & 55 & 15.32011 & e-1 & 193 \\
\hline
\end{tabular}

Totals : $\quad 1079.41936 \quad 25.57684$ 

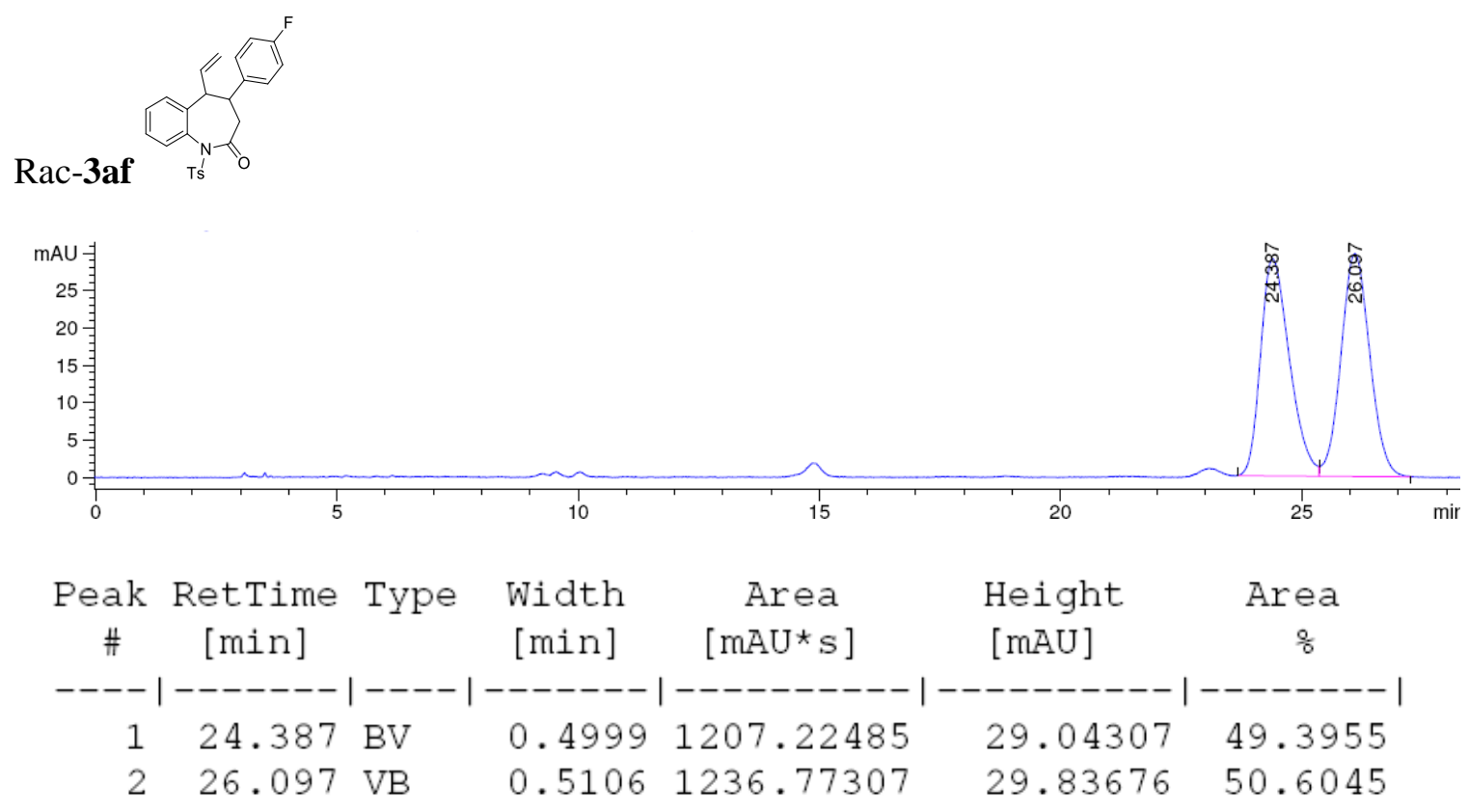

Totals : $\quad 2443.99792 \quad 58.87983$
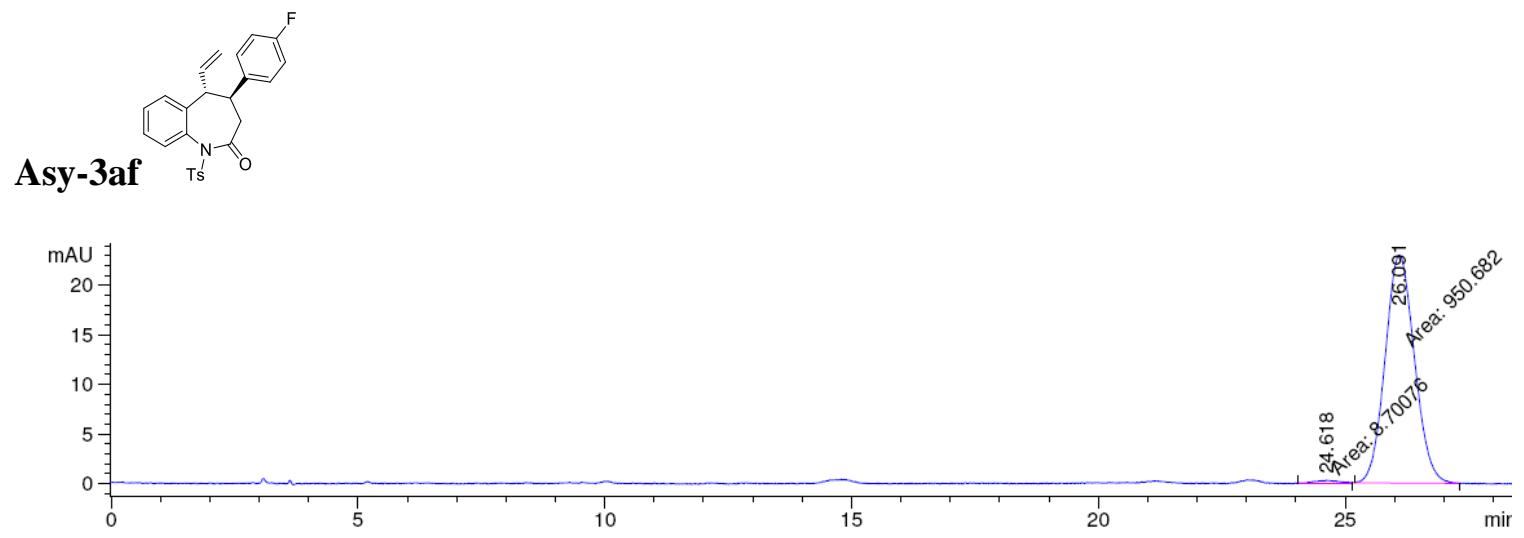

\begin{tabular}{|c|c|c|c|c|c|c|}
\hline $\begin{array}{c}\text { Peak } \\
\#\end{array}$ & $\begin{array}{c}\text { RetTime } \\
\text { [min] }\end{array}$ & Type & $\begin{array}{l}\text { Width } \\
\text { [min] }\end{array}$ & $\begin{array}{c}\text { Area } \\
{\left[\mathrm{mAU}{ }^{*} \mathrm{~s}\right]}\end{array}$ & $\begin{array}{l}\text { Height } \\
{[\mathrm{mAU}]}\end{array}$ & $\begin{array}{c}\text { Area } \\
\%\end{array}$ \\
\hline & & & & & & \\
\hline & & & & 076 & $\begin{array}{l}-1 \\
68\end{array}$ & \\
\hline
\end{tabular}

Totals :

$959.38301 \quad 23.34578$ 

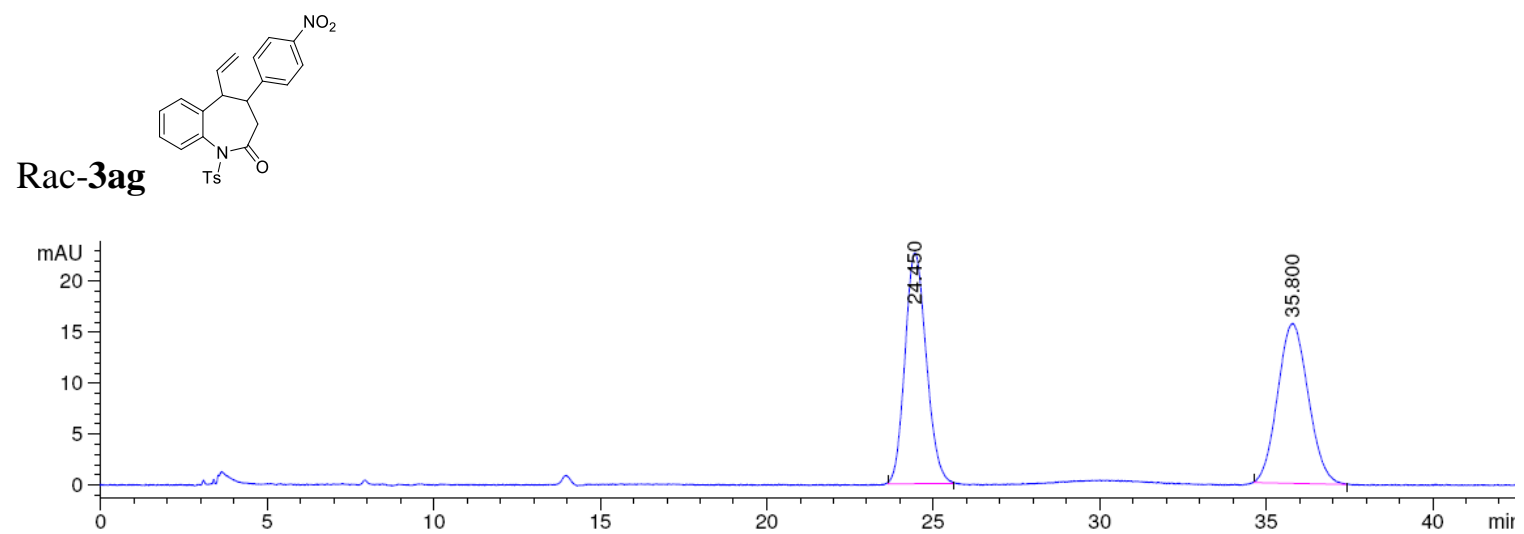

\begin{tabular}{|c|c|c|c|c|c|c|}
\hline $\begin{array}{c}\text { Peak } \\
\#\end{array}$ & $\begin{array}{c}\text { RetTime } \\
\text { [min] }\end{array}$ & Type & $\begin{array}{l}\text { Width } \\
\text { [min] }\end{array}$ & $\begin{array}{c}\text { Area } \\
{\left[\mathrm{mAU}^{*} \mathrm{~s}\right]}\end{array}$ & $\begin{array}{l}\text { Height } \\
{[\mathrm{mAU}]}\end{array}$ & $\begin{array}{c}\text { Area } \\
\%\end{array}$ \\
\hline & ----- & & & ---------- & --------- & ---1 \\
\hline 1 & r & BB & 5323 & 1010.91223 & 22.66866 & 946 \\
\hline 2 & 800 & $\mathrm{~B}$ & & 1003.07428 & .71658 & 3054 \\
\hline
\end{tabular}

Totals : $\quad 2013.98651 \quad 38.38524$
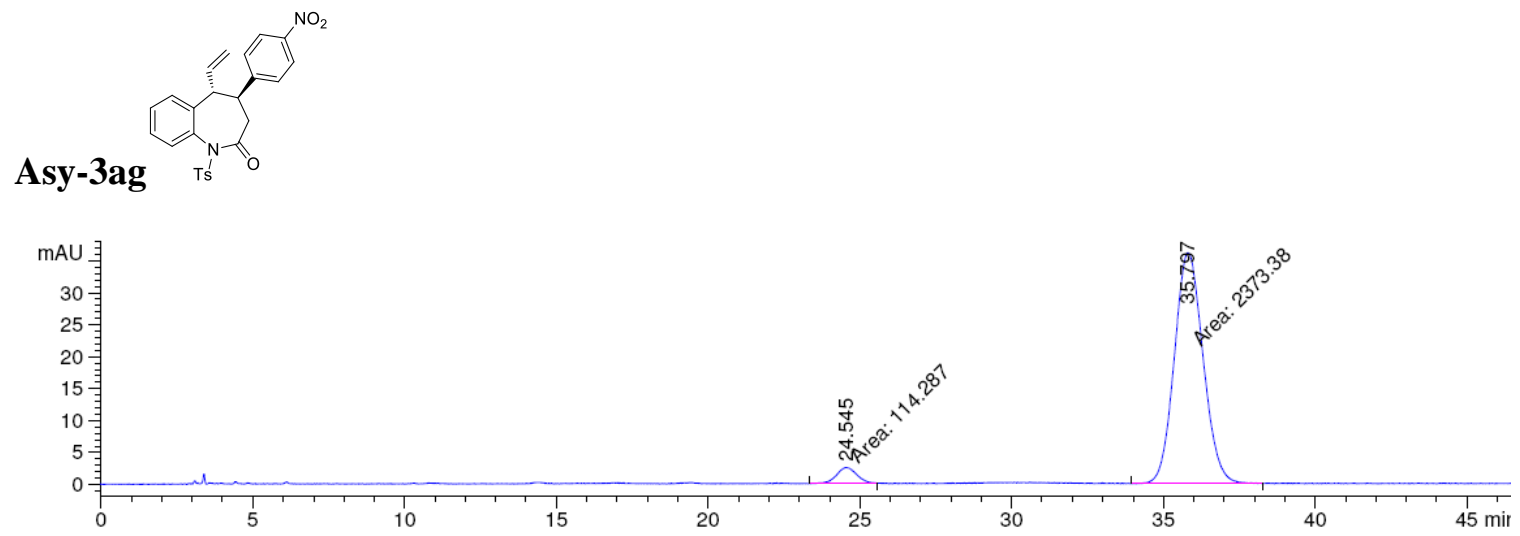

\begin{tabular}{|c|c|c|c|c|c|c|}
\hline $\begin{array}{c}\text { Peak } \\
\#\end{array}$ & $\begin{array}{c}\text { RetTime } \\
\text { [min] }\end{array}$ & Type & $\begin{array}{l}\text { Width } \\
\text { [min] }\end{array}$ & $\begin{array}{c}\text { Area } \\
{\left[\mathrm{mAU}^{\star} \mathrm{s}\right]}\end{array}$ & $\begin{array}{l}\text { Height } \\
{[\mathrm{mAU}]}\end{array}$ & $\begin{array}{c}\text { Area } \\
\%\end{array}$ \\
\hline & & & & --- & . & - \\
\hline 1 & & & & 114 & 905 & 41 \\
\hline 2 & 35.797 & $M$ & 3 & 2373.38330 & 6.08214 & 4059 \\
\hline
\end{tabular}

Totals: $\quad 2487.67004 \quad 38.57120$ 

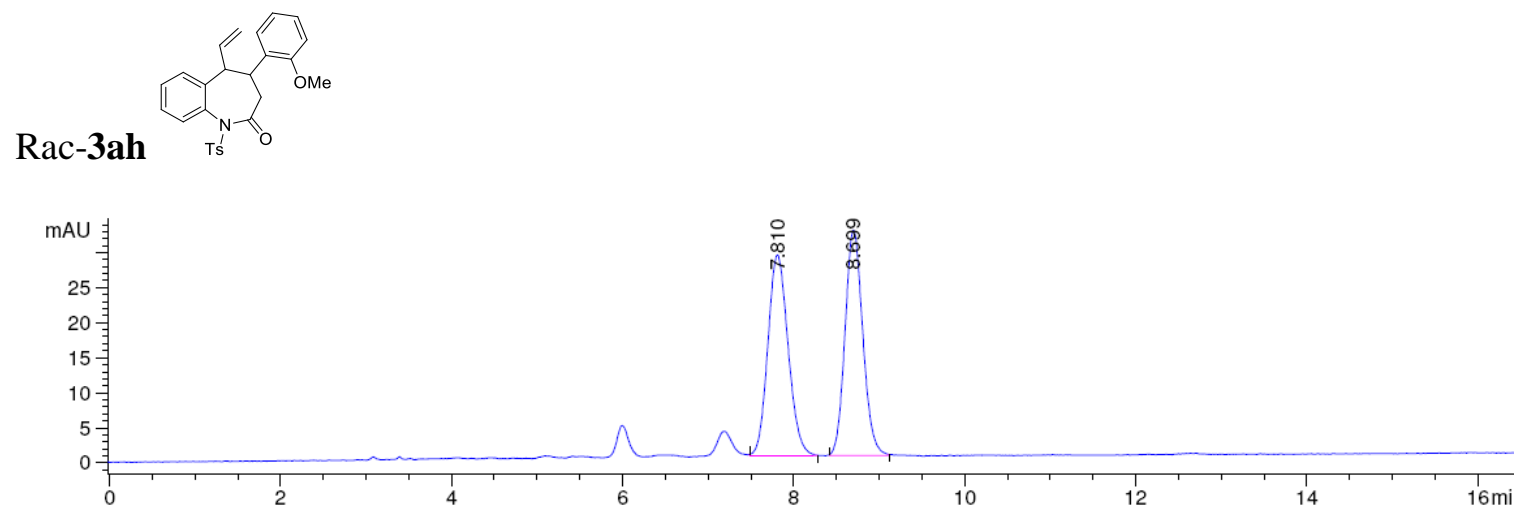

\begin{tabular}{|c|c|c|c|c|c|c|}
\hline $\begin{array}{c}\text { Peak } \\
\#\end{array}$ & $\begin{array}{c}\text { RetTime } \\
\text { [min] }\end{array}$ & Type & $\begin{array}{l}\text { Width } \\
\text { [min] }\end{array}$ & $\begin{array}{c}\text { Area } \\
{\left[\mathrm{mAU}^{*} \mathrm{~s}\right]}\end{array}$ & $\begin{array}{l}\text { Height } \\
{[\mathrm{mAU}]}\end{array}$ & $\begin{array}{c}\text { Area } \\
\frac{\%}{\delta}\end{array}$ \\
\hline-1 & & & & & & - \\
\hline 1 & 7 & $3 B$ & & 472.10971 & 19 & 04 \\
\hline 2 & 8.699 & תמ & & 463.49512 & 32.13346 & 5396 \\
\hline
\end{tabular}

$\begin{array}{lll}\text { Totals : } & 935.60483 \quad 60.77966\end{array}$
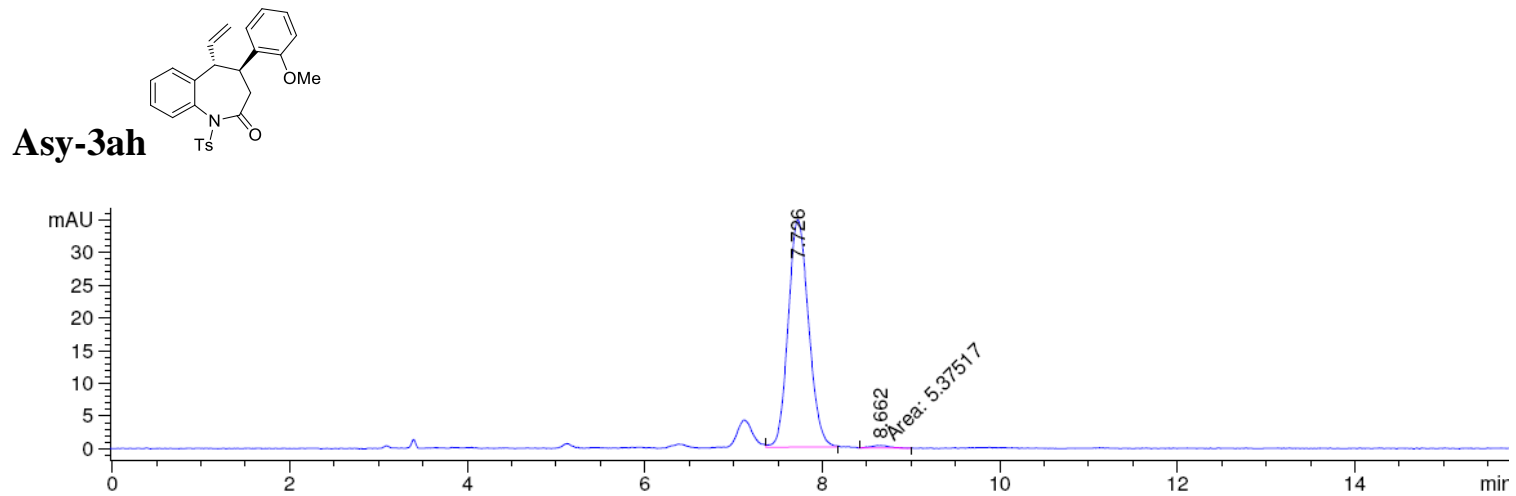

\begin{tabular}{|c|c|c|c|c|c|c|}
\hline $\begin{array}{c}\text { Peak } \\
\#\end{array}$ & $\begin{array}{c}\text { RetTime } \\
\text { [min] }\end{array}$ & Type & $\begin{array}{l}\text { Width } \\
\text { [min] }\end{array}$ & $\begin{array}{c}\text { Area } \\
{\left[\mathrm{mAU}^{*} \mathrm{~s}\right]}\end{array}$ & $\begin{array}{l}\text { Height } \\
{[\mathrm{mAU}]}\end{array}$ & $\begin{array}{c}\text { Area } \\
\%\end{array}$ \\
\hline & & & & . & 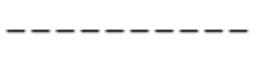 & -- \\
\hline 1 & 7.7 & $\mathrm{VB}$ & & 555.52563 & 362 & 117 \\
\hline 2 & 8.662 & $M M$ & 2 & 5.37517 & $71385 e-1$ & 9583 \\
\hline
\end{tabular}

Totals :

$560.90080 \quad 35.10500$ 

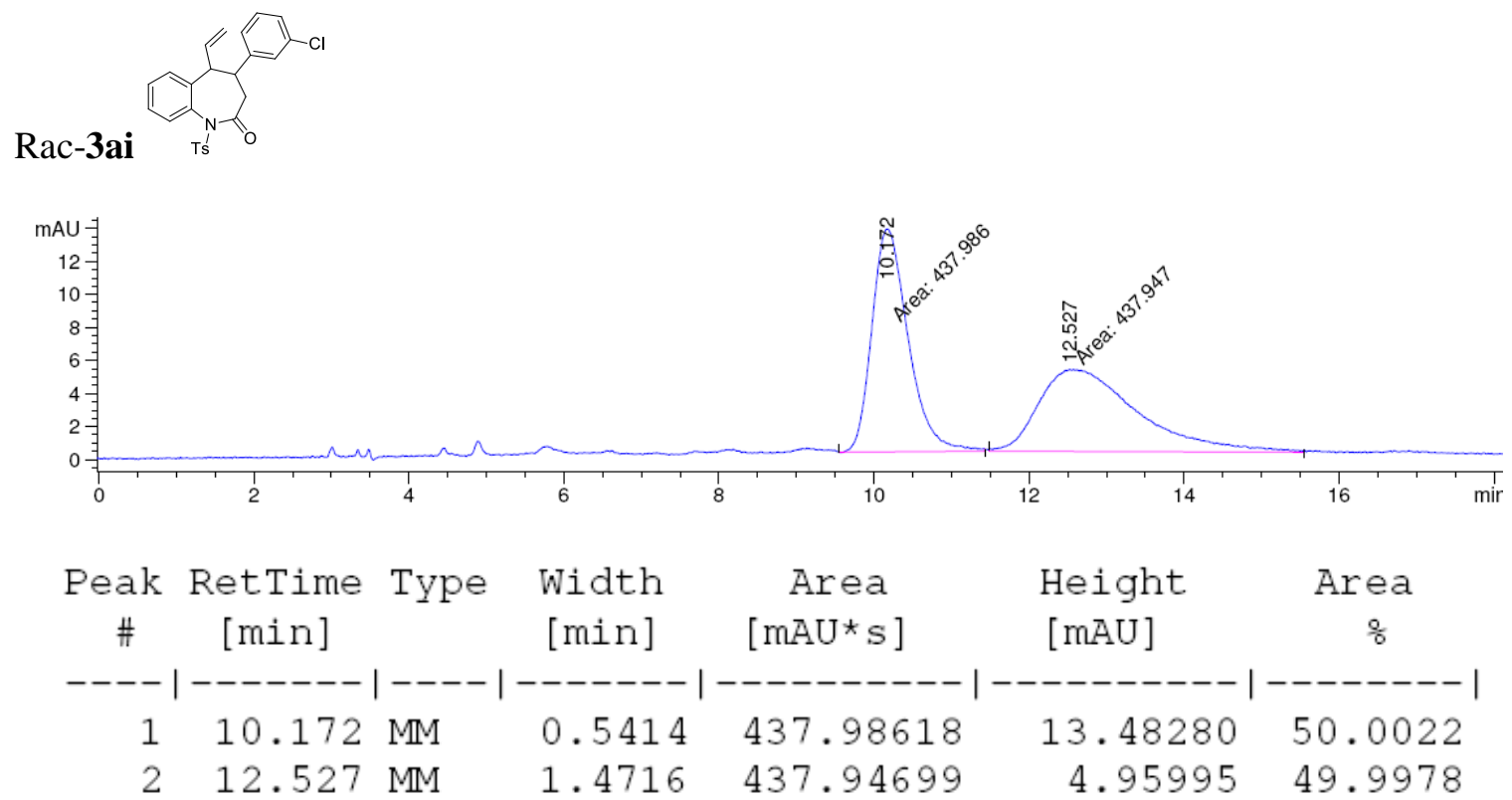

Totals : $\quad 875.93317 \quad 18.44275$
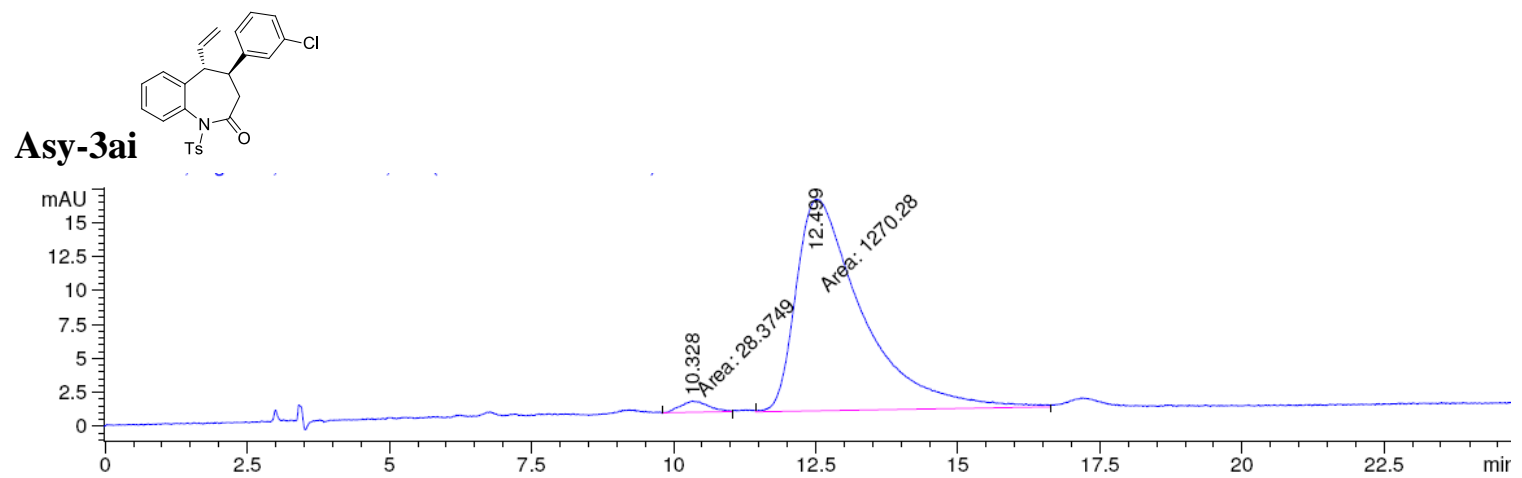

\begin{tabular}{|c|c|c|c|c|c|c|}
\hline $\begin{array}{c}\text { Peak } \\
\#\end{array}$ & $\begin{array}{c}\text { RetTime } \\
\text { [min] }\end{array}$ & Type & $\begin{array}{l}\text { Width } \\
\text { [min] }\end{array}$ & $\begin{array}{c}\text { Area } \\
{\left[\mathrm{mAU}^{\star} \mathrm{s}\right]}\end{array}$ & $\begin{array}{l}\text { Height } \\
\text { [mAU] }\end{array}$ & $\begin{array}{c}\text { Area } \\
\%\end{array}$ \\
\hline & & & & & & \\
\hline 2 & 12.499 & $\mathrm{MM}$ & 3562 & 1270.27722 & 15.61026 & 3150 \\
\hline
\end{tabular}

Totals :

$1298.65213 \quad 16.43720$ 

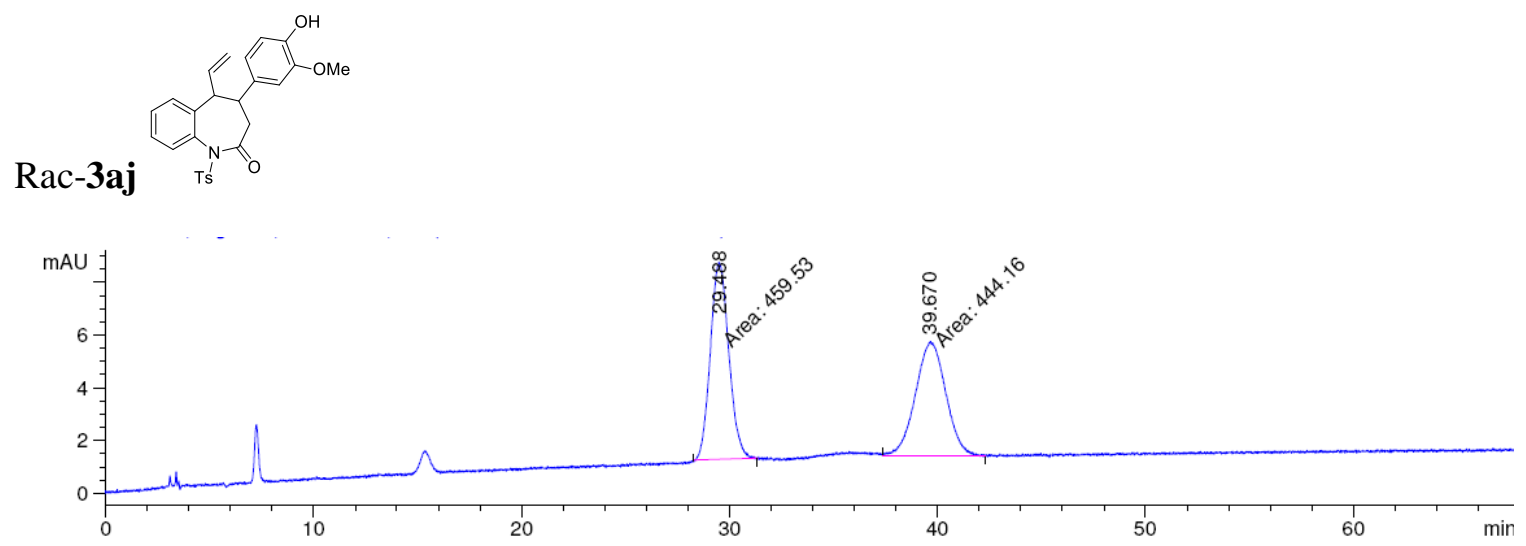

\begin{tabular}{|c|c|c|c|c|c|c|}
\hline $\begin{array}{c}\text { Peak } \\
\#\end{array}$ & $\begin{array}{c}\text { RetTime } \\
\text { [min] }\end{array}$ & Type & $\begin{array}{l}\text { Width } \\
\text { [min] }\end{array}$ & $\begin{array}{c}\text { Area } \\
{\left[\mathrm{mAU}{ }^{*} \mathrm{~s}\right]}\end{array}$ & $\begin{array}{l}\text { Height } \\
{[m A U]}\end{array}$ & $\begin{array}{c}\text { Area } \\
\%\end{array}$ \\
\hline & & & & 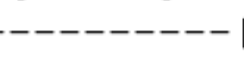 & & \\
\hline 1 & $a$ & $\mathbb{I}^{4}$ & 1.0240 & 459.53027 & 7.4 & 04 \\
\hline 2 & 39.670 & $\mathrm{MM}$ & 1.7010 & 444.16028 & 4.35193 & 49.1496 \\
\hline
\end{tabular}

Totals : $\quad 903.69055 \quad 11.83103$
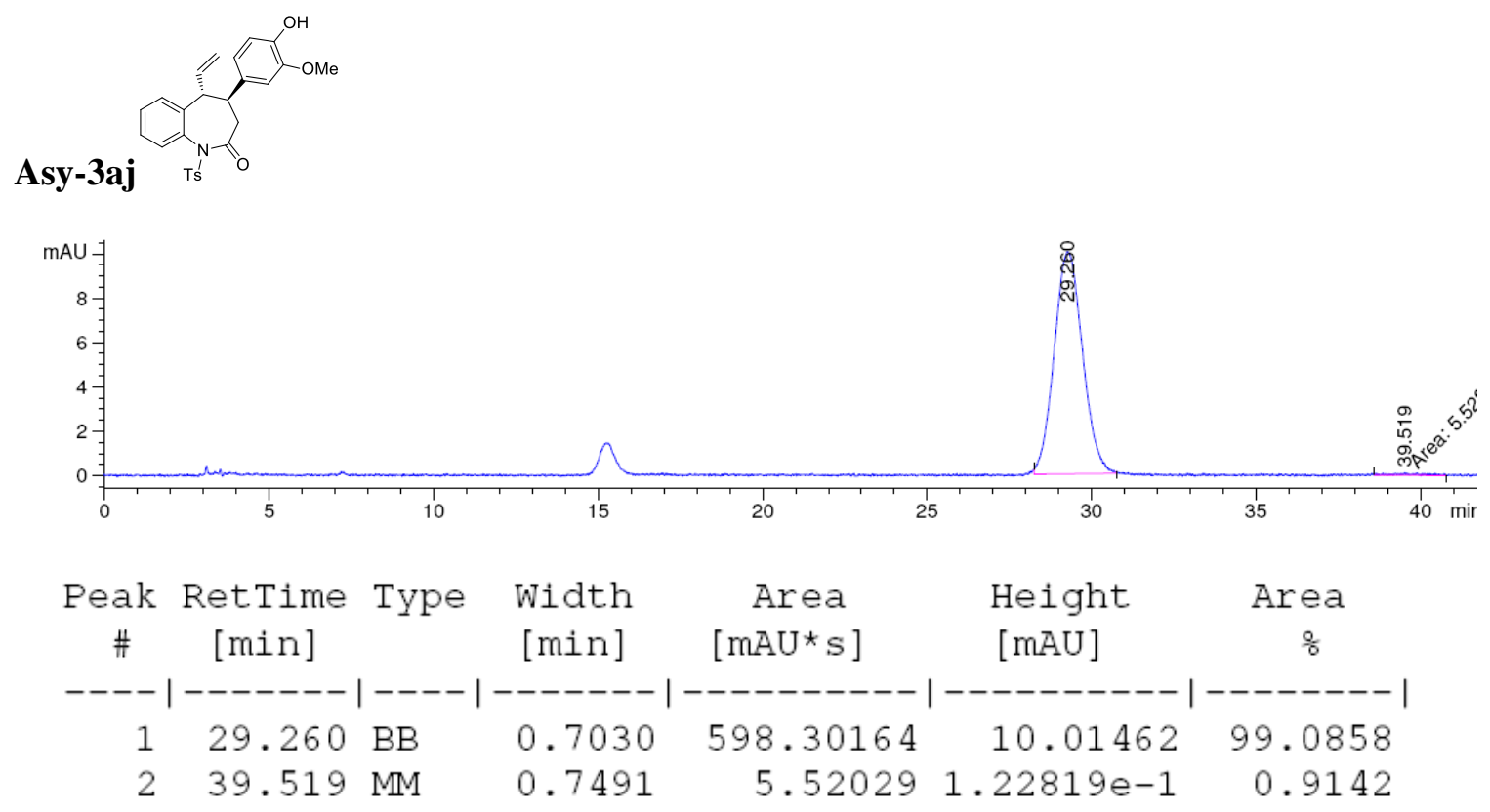

Totals :

$603.82193 \quad 10.13743$ 

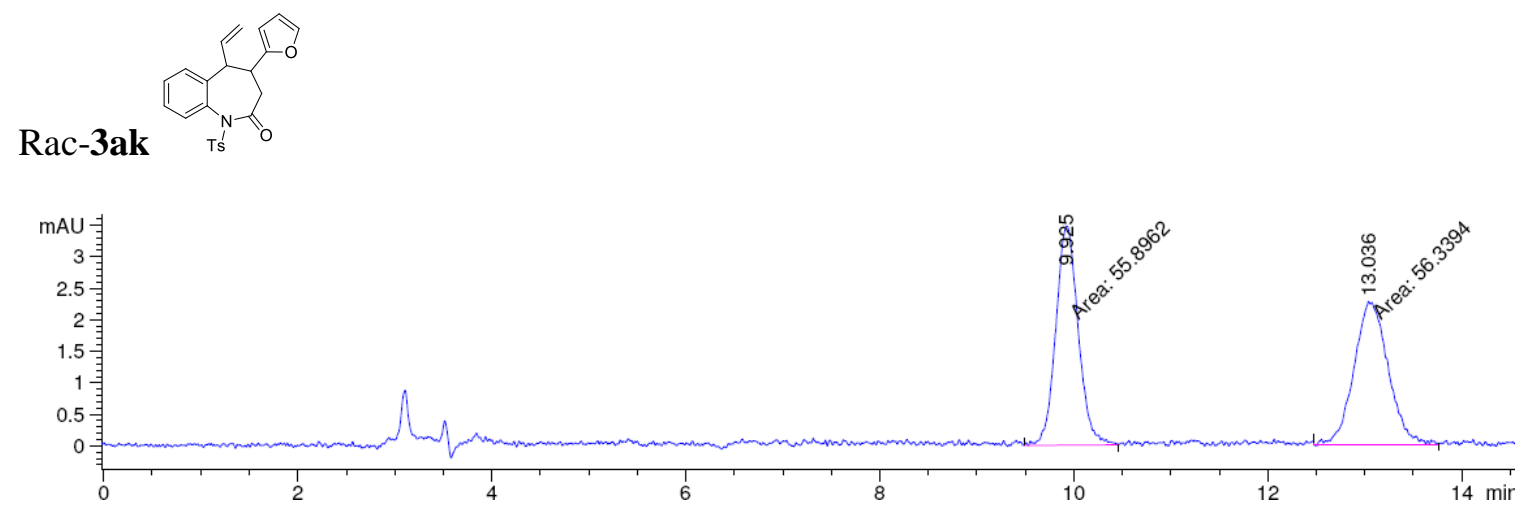

\begin{tabular}{|c|c|c|c|c|c|c|}
\hline $\begin{array}{c}\text { Peak } \\
\#\end{array}$ & $\begin{array}{c}\text { RetTime } \\
\text { [min] }\end{array}$ & Type & $\begin{array}{l}\text { Width } \\
\text { [min] }\end{array}$ & $\begin{array}{c}\text { Area } \\
{\left[\mathrm{mAU}^{\star} \mathrm{s}\right]}\end{array}$ & $\begin{array}{l}\text { Height } \\
{[\mathrm{mAU}]}\end{array}$ & $\begin{array}{c}\text { Area } \\
\%\end{array}$ \\
\hline 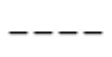 & & & & ------- & & 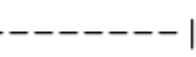 \\
\hline 1 & 9.92 & $M$ & & 9616 & 593 & 25 \\
\hline ? & 13. & $M \mathrm{M}$ & & 56.33940 & .28089 & 1975 \\
\hline
\end{tabular}
Totals :
112.23556
5.75682
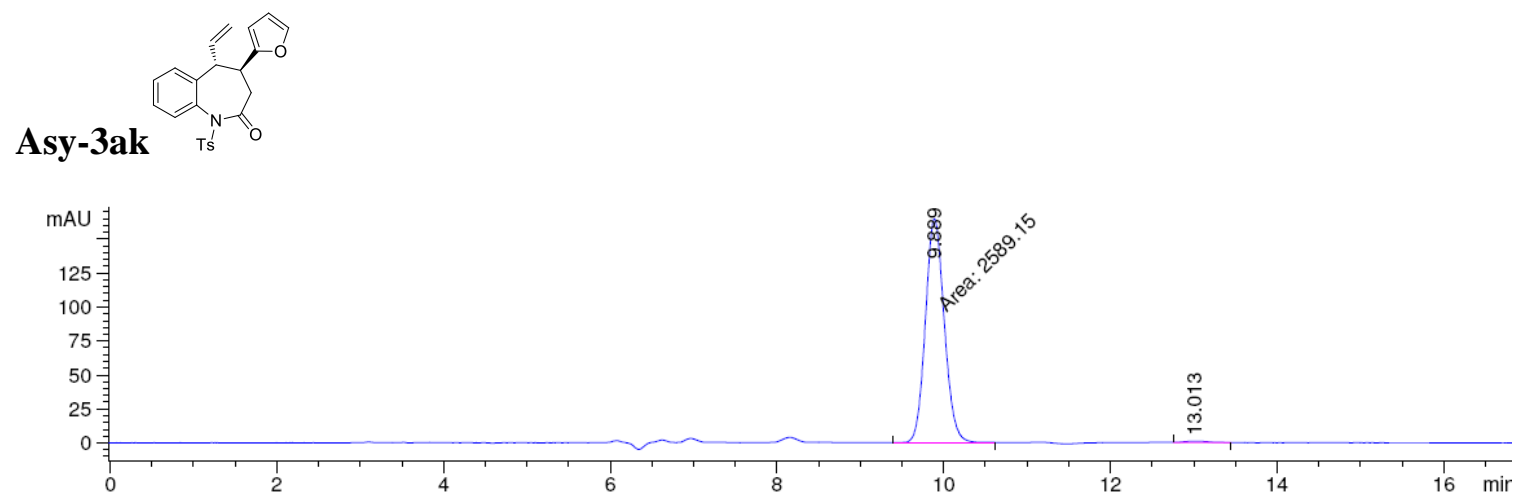

\begin{tabular}{|c|c|c|c|c|c|c|}
\hline $\begin{array}{c}\text { Peak } \\
\#\end{array}$ & $\begin{array}{c}\text { RetTime } \\
\text { [min] }\end{array}$ & Type & $\begin{array}{l}\text { Width } \\
\text { [min] }\end{array}$ & $\begin{array}{c}\text { Area } \\
{\left[\mathrm{mAU}^{*} \mathrm{~s}\right]}\end{array}$ & $\begin{array}{l}\text { Height } \\
{[\mathrm{mAU}]}\end{array}$ & $\begin{array}{c}\text { Area } \\
\%\end{array}$ \\
\hline & & & & --- & 1 & \\
\hline 1 & a & $\mathrm{MM}$ & & 2589.14551 & 164. & 99 \\
\hline 2 & 13.013 & $B R$ & 0 . & 22.01833 & 1.04977 & 43 \\
\hline
\end{tabular}

Totals :

$2611.16384 \quad 165.80845$ 

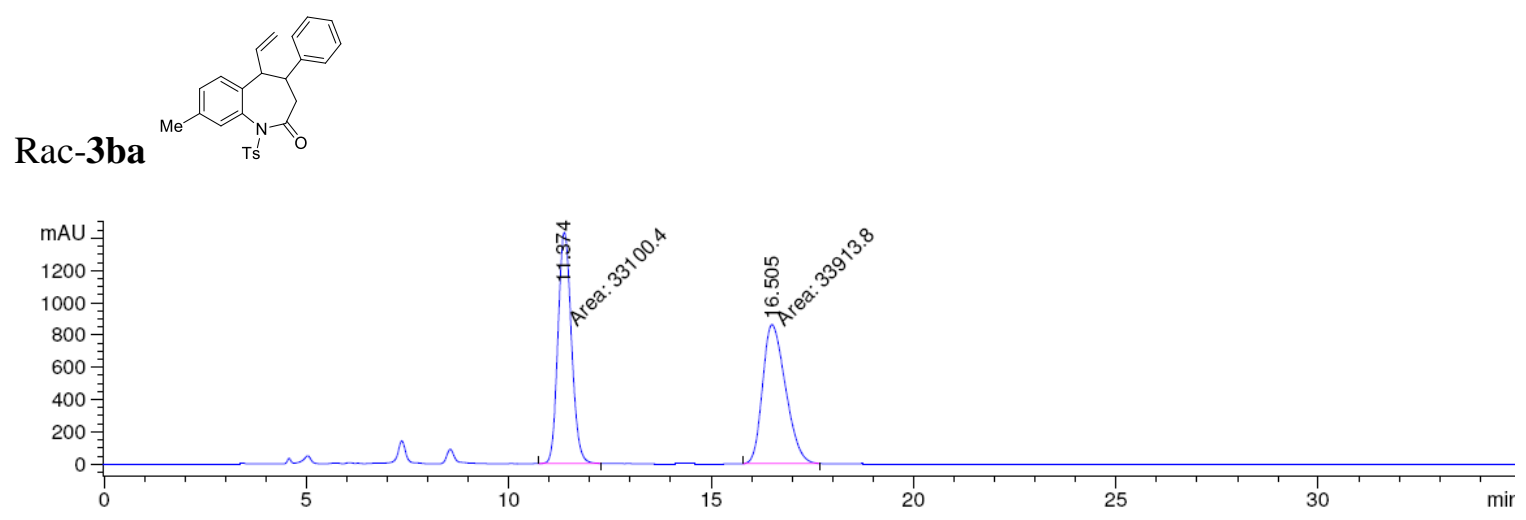

\begin{tabular}{|c|c|c|c|c|c|c|}
\hline $\begin{array}{c}\text { Peak } \\
\#\end{array}$ & $\begin{array}{c}\text { RetTime } \\
\text { [min] }\end{array}$ & Type & $\begin{array}{l}\text { Width } \\
\text { [min] }\end{array}$ & $\begin{array}{c}\text { Area } \\
{\left[\mathrm{mAU}^{*} \mathrm{~s}\right]}\end{array}$ & $\begin{array}{l}\text { Height } \\
{[\mathrm{mAU}]}\end{array}$ & $\begin{array}{c}\text { Area } \\
\frac{\%}{6}\end{array}$ \\
\hline & & & & & & -------1 \\
\hline 1 & 11.37 & MM & $0 \quad 30$ & 31004 e 4 & 1432.16418 & 49.3931 \\
\hline 2 & 16.505 & MM & 562 & 3. $39138 e 4$ & 861.32251 & 50.6069 \\
\hline
\end{tabular}

Totals : $\quad 6.70142 e 4 \quad 2293.48669$
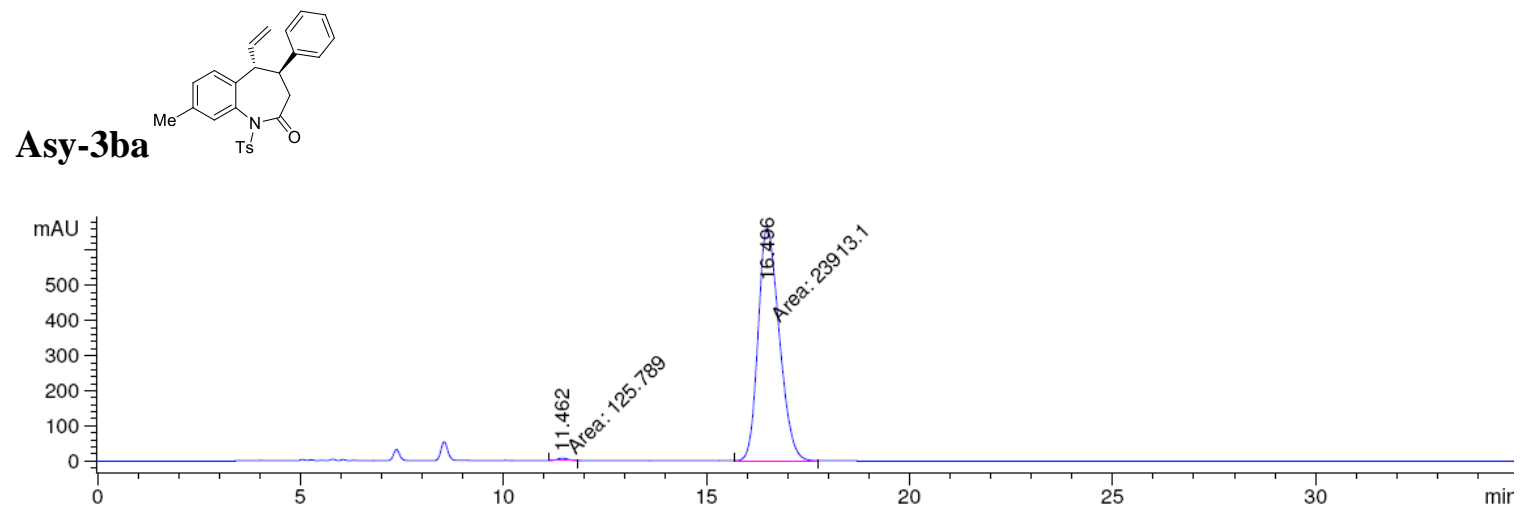

\begin{tabular}{|c|c|c|c|c|c|c|}
\hline $\begin{array}{c}\text { eak } \\
\#\end{array}$ & $\begin{array}{c}\text { RetTime } \\
\text { [min] }\end{array}$ & Type & $\begin{array}{c}\text { Width } \\
{[\mathrm{min}]}\end{array}$ & $\begin{array}{c}\text { Area } \\
{\left[\mathrm{mAU}^{\star} \mathrm{s}\right]}\end{array}$ & $\begin{array}{c}\text { Height } \\
{[\mathrm{mAU}]}\end{array}$ & $\begin{array}{c}\text { Area } \\
\%\end{array}$ \\
\hline & & & & & -- & \\
\hline 1 & & 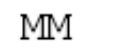 & & 3862 & 95 & \\
\hline 2 & 496 & FII & 16 & $2.39131 e 4$ & 662.43146 & 4767 \\
\hline
\end{tabular}

Totals : $\quad 2.40389 e 4 \quad 669.25441$ 

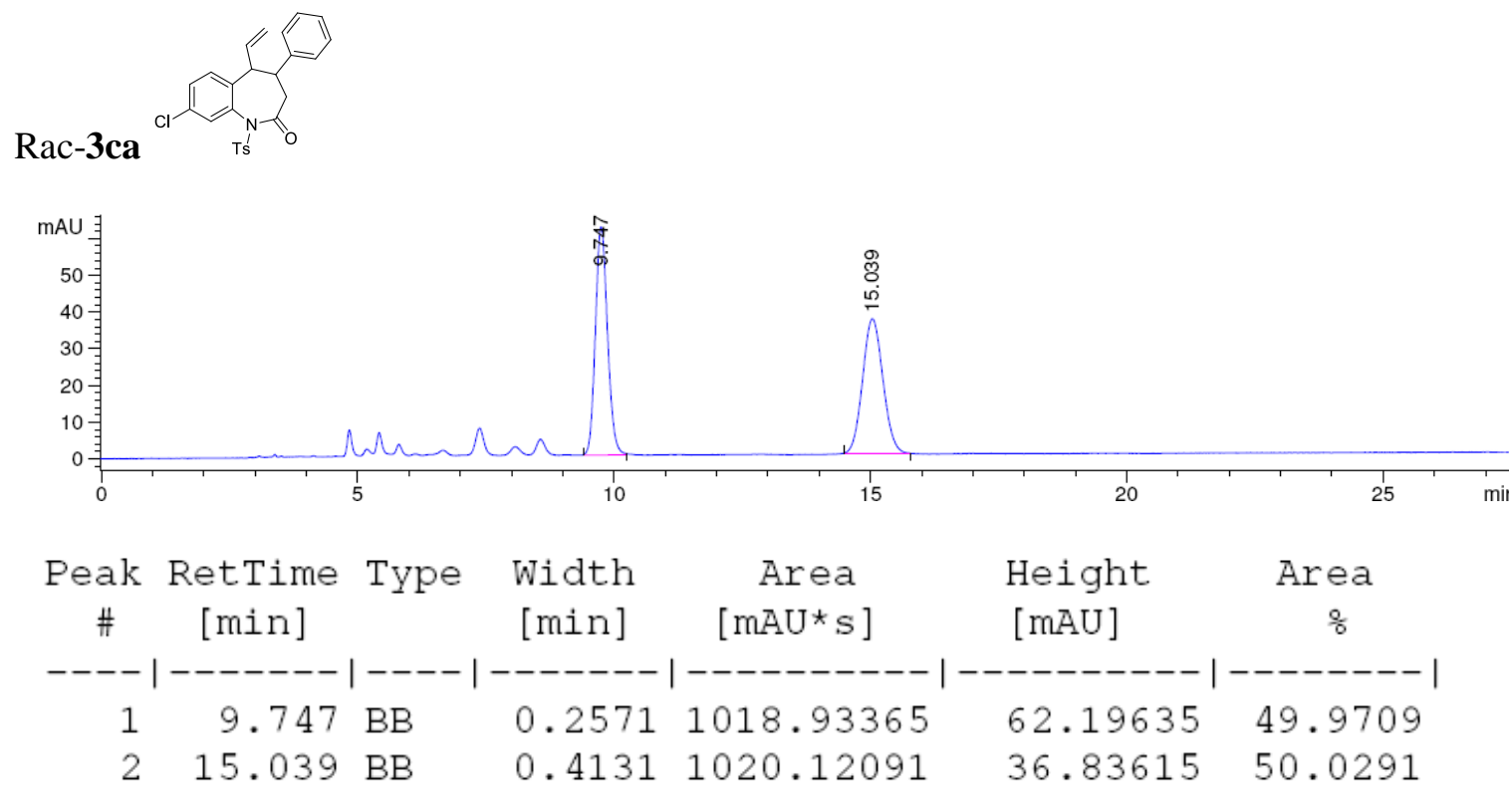

Totals : $\quad 2039.05457 \quad 99.03251$
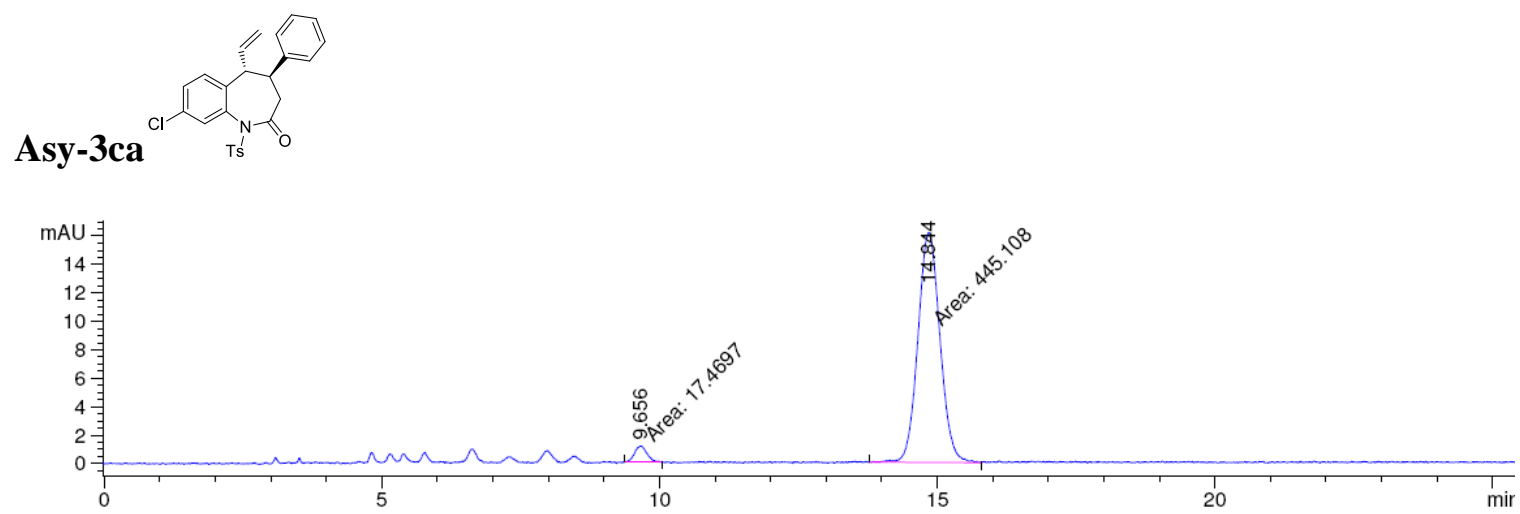

\begin{tabular}{|c|c|c|c|c|c|c|}
\hline $\begin{array}{c}\text { Peak } \\
\#\end{array}$ & $\begin{array}{c}\text { RetTime } \\
\text { [min] }\end{array}$ & Type & $\begin{array}{l}\text { Width } \\
\text { [min] }\end{array}$ & $\begin{array}{c}\text { Area } \\
{\left[\mathrm{mAU}{ }^{*} \mathrm{~s}\right]}\end{array}$ & $\begin{array}{l}\text { Height } \\
{[\mathrm{mAU}]}\end{array}$ & $\begin{array}{c}\text { Area } \\
\%\end{array}$ \\
\hline & & & & & & \\
\hline$\frac{1}{2}$ & 14.844 & . & & $\begin{array}{r}17.46974 \\
445.10800\end{array}$ & $\begin{array}{l}2392 \\
6714\end{array}$ & 2234 \\
\hline
\end{tabular}

Totals :

$462.57774 \quad 17.29106$ 

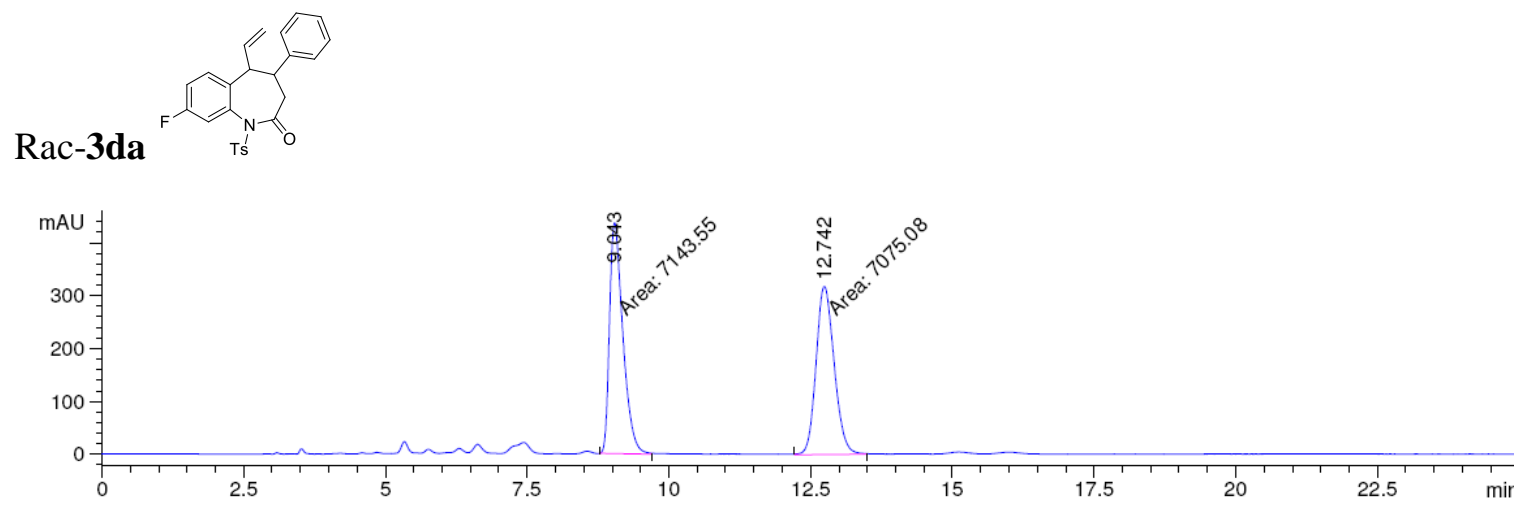

\begin{tabular}{|c|c|c|c|c|c|c|}
\hline $\begin{array}{c}\text { Peak } \\
\#\end{array}$ & $\begin{array}{c}\text { RetTime } \\
\text { [min] }\end{array}$ & Type & $\begin{array}{l}\text { Width } \\
\text { [min] }\end{array}$ & $\begin{array}{c}\text { Area } \\
{\left[\mathrm{mAU}^{\star} \mathrm{s}\right]}\end{array}$ & $\begin{array}{l}\text { Height } \\
{[m A U]}\end{array}$ & $\begin{array}{c}\text { Area } \\
\%\end{array}$ \\
\hline & & & & ---------- & ---- & $=1$ \\
\hline 1 & a & $M$ & 27 & 7143.54932 & 55115 & 408 \\
\hline 2 & 12.742 & M & 06 & 7075.07617 & 318.15292 & 592 \\
\hline
\end{tabular}

Totals : $\quad 1.42186 e 4 \quad 754.70407$
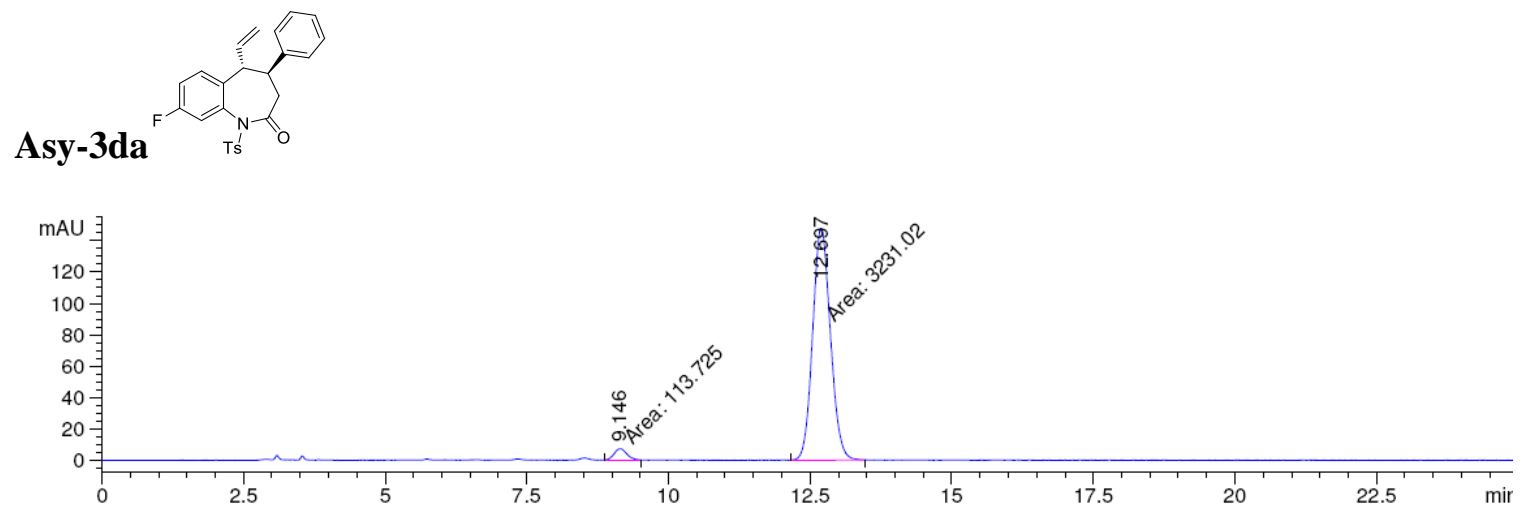

\begin{tabular}{|c|c|c|c|c|c|c|}
\hline $\begin{array}{c}\text { eak } \\
\#\end{array}$ & $\begin{array}{c}\text { RetTime } \\
\text { [min] }\end{array}$ & Type & $\begin{array}{l}\text { Width } \\
\text { [min] }\end{array}$ & $\begin{array}{c}\text { Area } \\
{\left[\mathrm{mAU}^{*} \mathrm{~s}\right]}\end{array}$ & $\begin{array}{l}\text { Height } \\
{[\mathrm{mAU}]}\end{array}$ & $\begin{array}{c}\text { Area } \\
\%\end{array}$ \\
\hline & & & & & & \\
\hline 1 & & & & & & \\
\hline 2 & 12.697 & 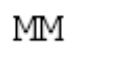 & .3642 & 3231.01904 & 147.86346 & 96.5999 \\
\hline
\end{tabular}

Totals :

$3344.74381 \quad 155.14368$ 

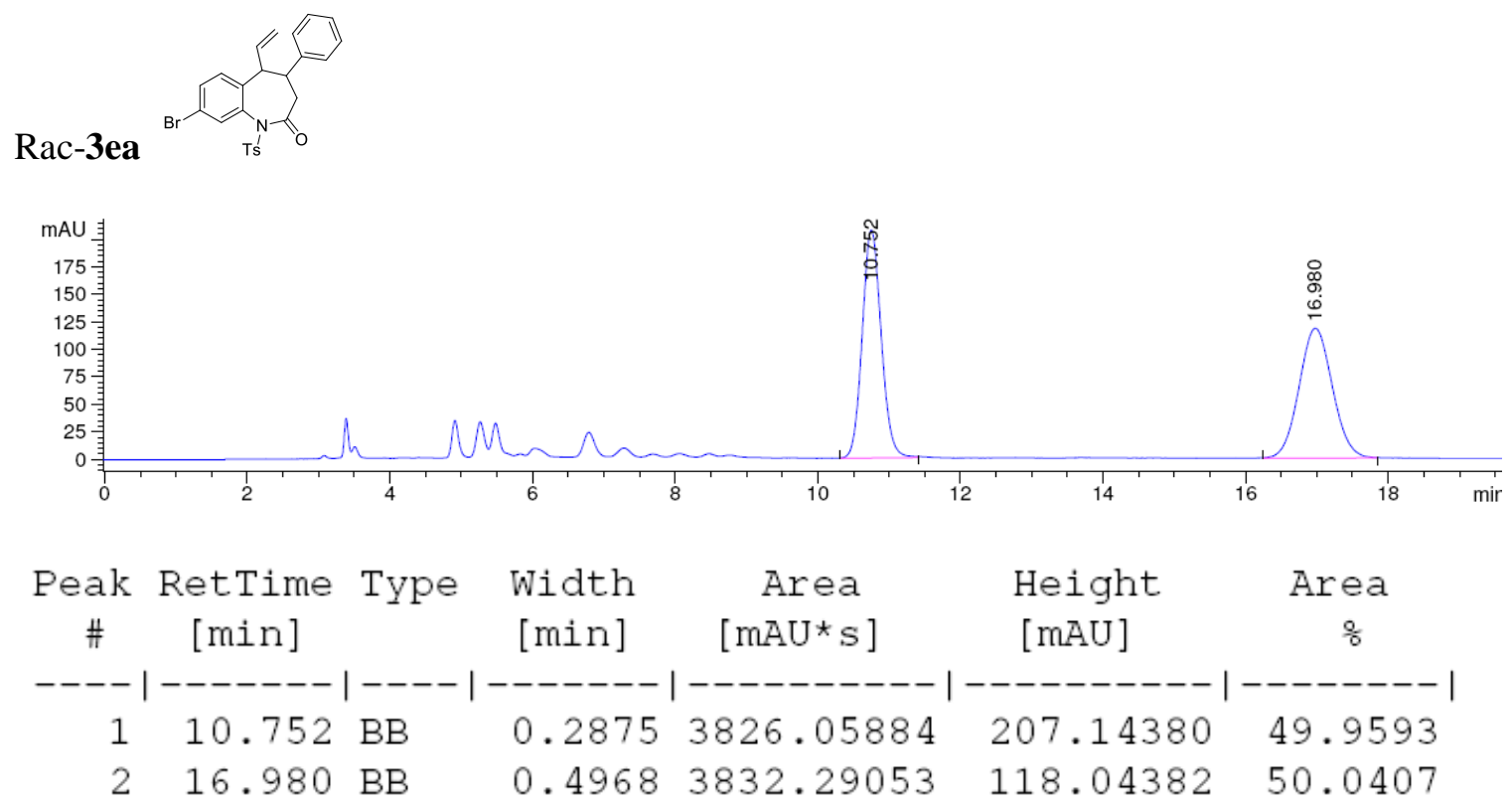

Totals :

$7658.34937 \quad 325.18761$
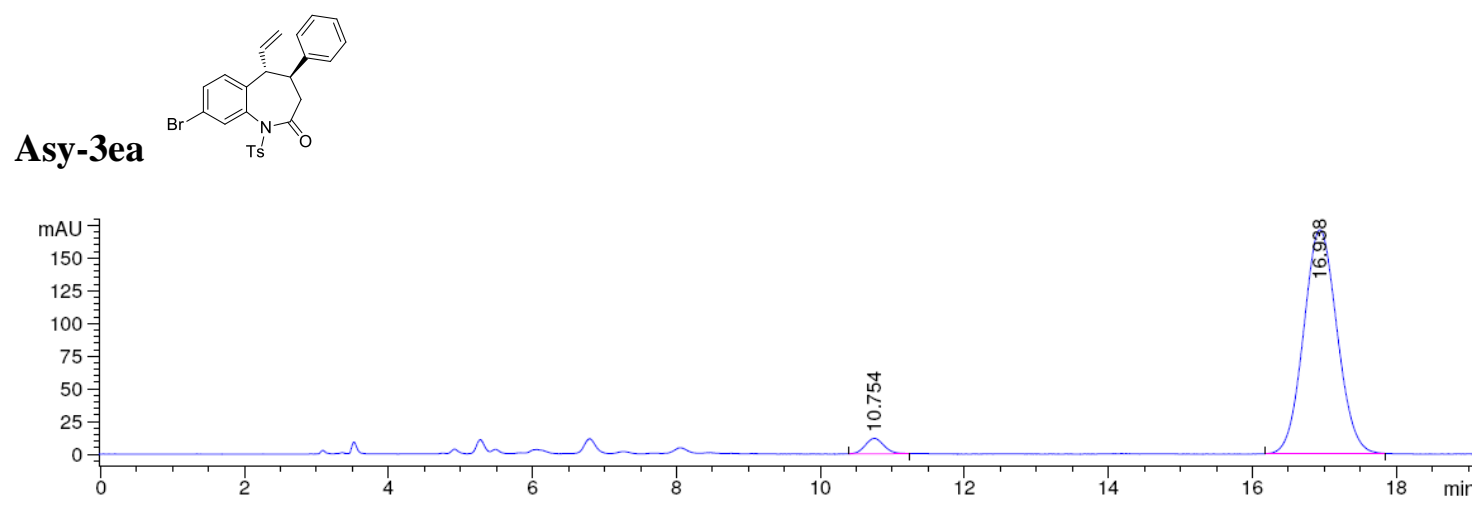

\begin{tabular}{|c|c|c|c|c|c|c|}
\hline $\begin{array}{c}\text { Peak } \\
\#\end{array}$ & $\begin{array}{c}\text { RetTime } \\
\text { [min] }\end{array}$ & Type & $\begin{array}{l}\text { Width } \\
\text { [min] }\end{array}$ & $\begin{array}{c}\text { Area } \\
{\left[\mathrm{mAU}^{\star} \mathrm{s}\right]}\end{array}$ & $\begin{array}{l}\text { Height } \\
{[\mathrm{mAU}]}\end{array}$ & $\begin{array}{c}\text { Area } \\
\%\end{array}$ \\
\hline & & & & & & \\
\hline 1 & & & 0 & 213.20137 & & \\
\hline 2 & 16.938 & $B$ & .4962 & 5545.49463 & 171.49783 & 96.2977 \\
\hline
\end{tabular}

Totals :

$5758.69600 \quad 183.29112$ 

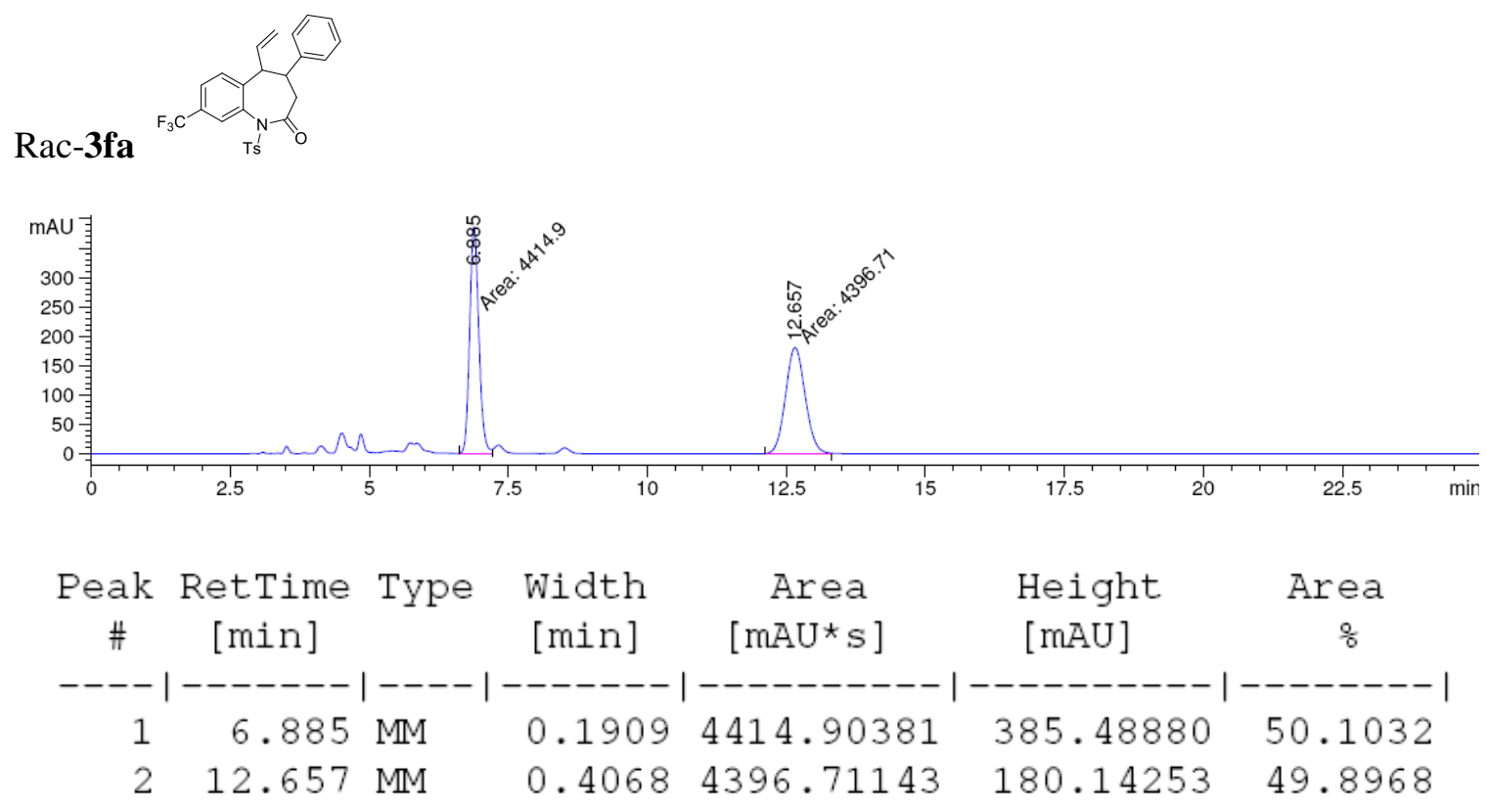

Totals :

$8811.61523 \quad 565.63133$
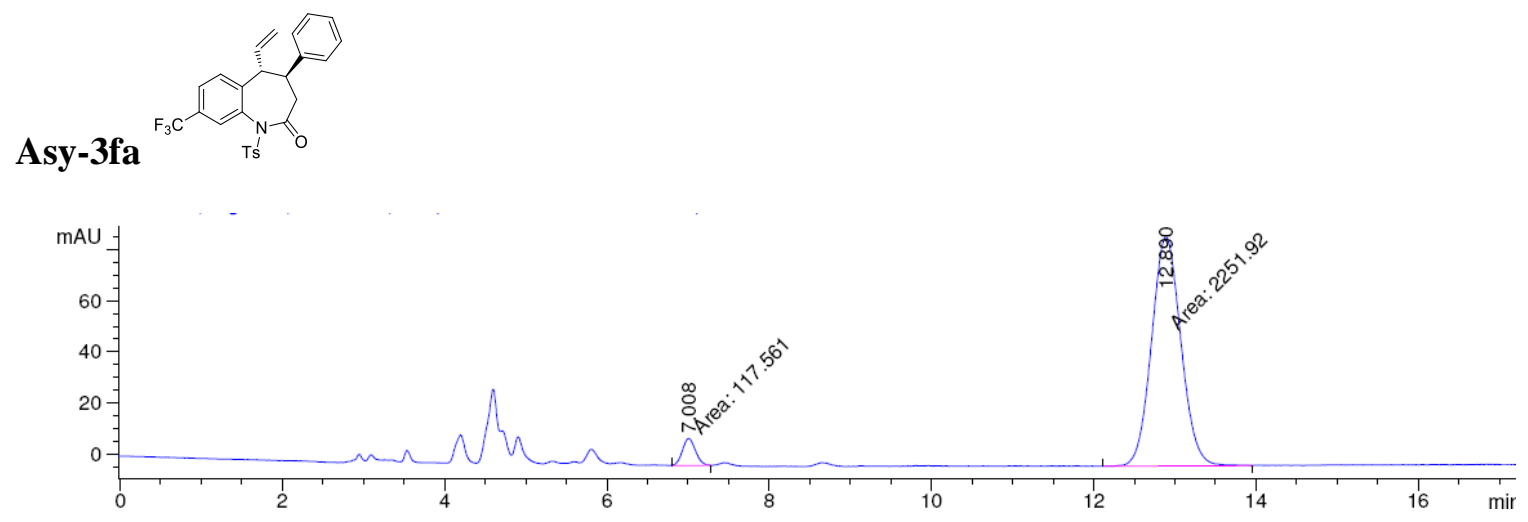

\begin{tabular}{|c|c|c|c|c|c|c|}
\hline $\begin{array}{c}\text { Peak } \\
\#\end{array}$ & $\begin{array}{c}\text { RetTime } \\
\text { [min] }\end{array}$ & Type & $\begin{array}{c}\text { Width } \\
\text { [min] }\end{array}$ & $\begin{array}{c}\text { Area } \\
{\left[\mathrm{mAU}^{*} \mathrm{~s}\right]}\end{array}$ & $\begin{array}{l}\text { Height } \\
{[\mathrm{mAU}]}\end{array}$ & $\begin{array}{c}\text { Area } \\
\%\end{array}$ \\
\hline & ----- & & ------- & ---------- & --------- & $-------\mid$ \\
\hline 1 & 7.008 & MM & 0.1864 & 117.56094 & 10.50970 & 4.9615 \\
\hline 2 & 12.890 & $\mathrm{MM}$ & 0.4197 & 2251.91626 & 89.42966 & 95.0385 \\
\hline
\end{tabular}

Totals : $\quad 2369.47720 \quad 99.93936$ 

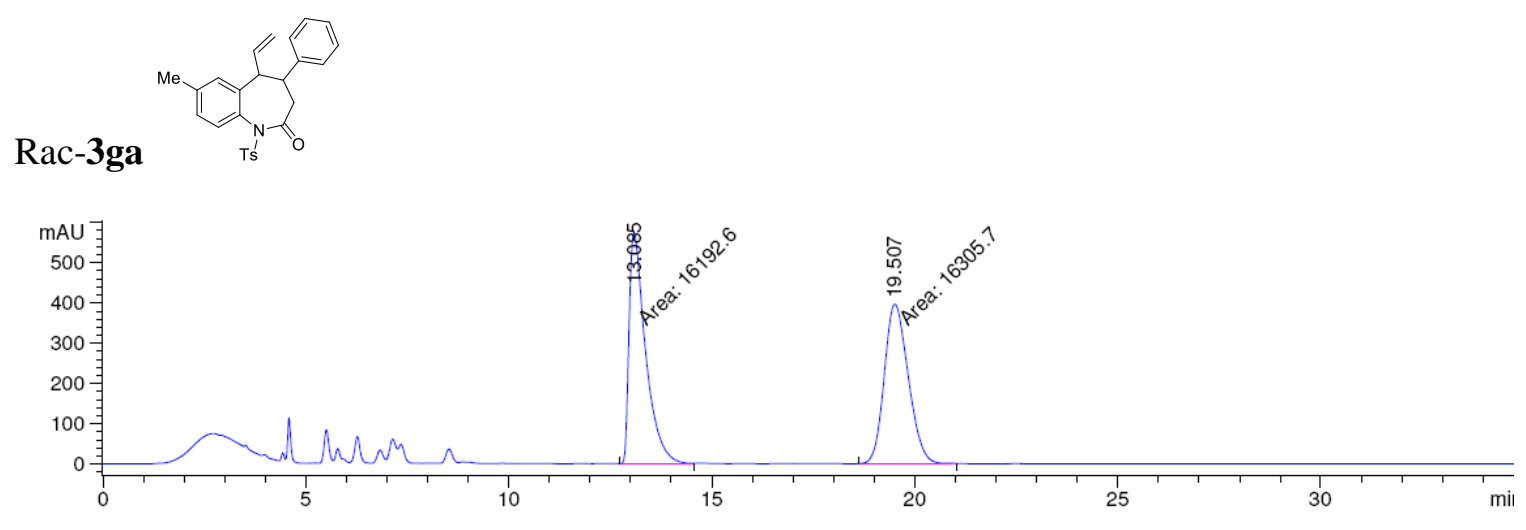

\begin{tabular}{|c|c|c|c|c|c|c|}
\hline $\begin{array}{c}\text { Peak } \\
\#\end{array}$ & $\begin{array}{c}\text { RetTime } \\
\text { [min] }\end{array}$ & Type & $\begin{array}{l}\text { Width } \\
\text { [min] }\end{array}$ & $\begin{array}{c}\text { Area } \\
{\left[\mathrm{mAU}^{*} \mathrm{~s}\right]}\end{array}$ & $\begin{array}{l}\text { Height } \\
{[\mathrm{mAU}]}\end{array}$ & $\begin{array}{c}\text { Area } \\
\%\end{array}$ \\
\hline & & & & & -- & 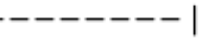 \\
\hline 1 & 3.085 & M & & $1.61926 e 4$ & 574.35217 & 60 \\
\hline 2 & 19.507 & MM & .6859 & $1.63057 e 4$ & 396.19531 & 1740 \\
\hline
\end{tabular}

Totals : $\quad 3.24983 e 4 \quad 970.54749$
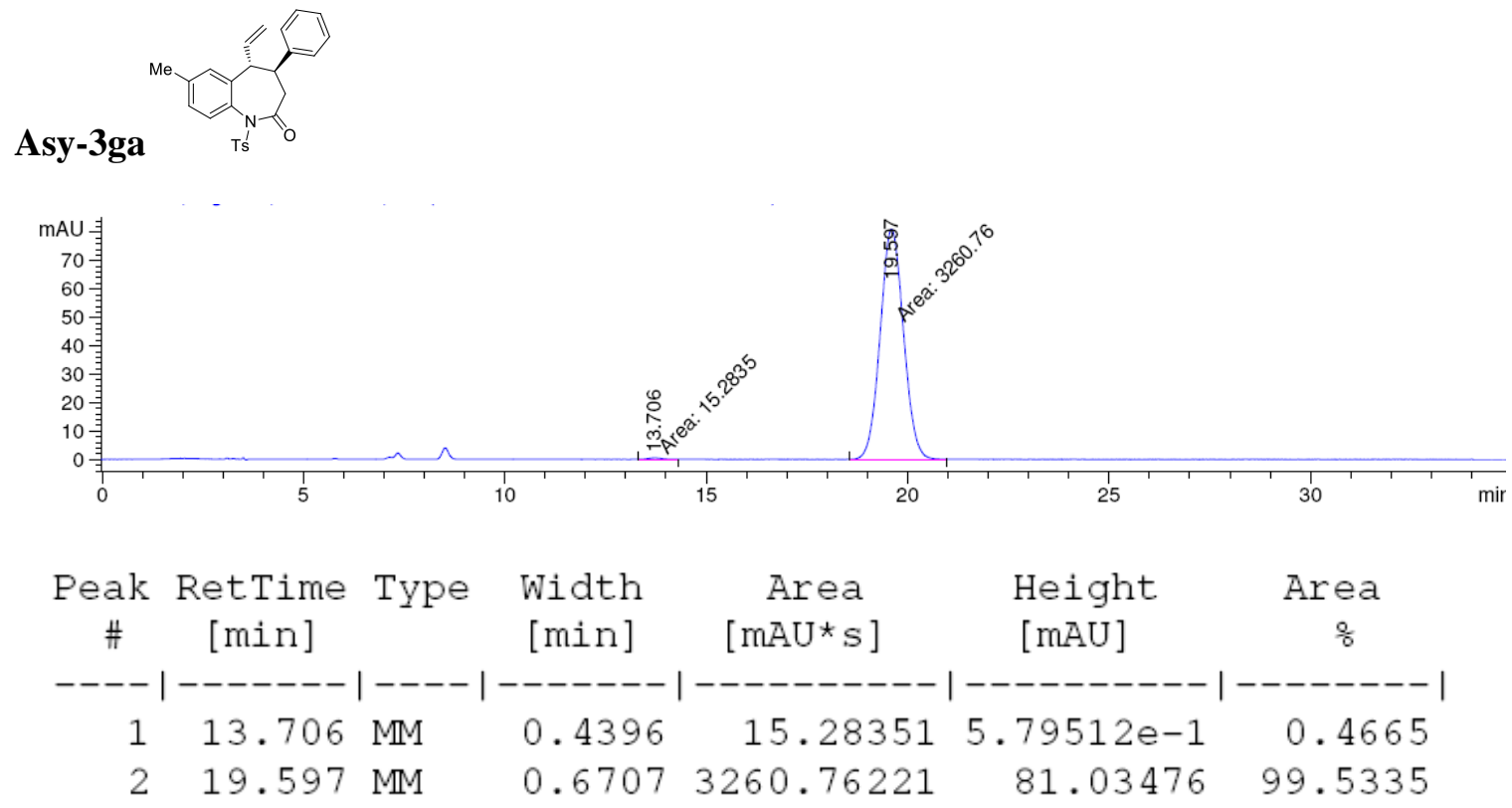

$\begin{array}{lll}\text { Totals : } & 3276.04572 \quad 81.61427\end{array}$ 

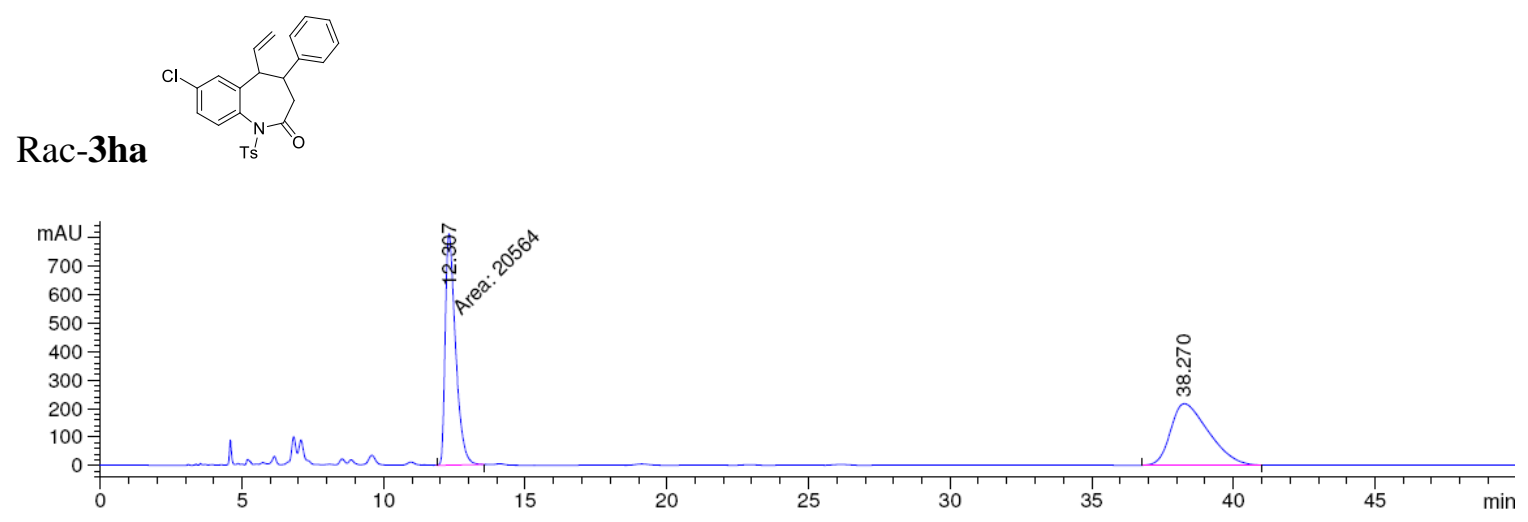

\begin{tabular}{|c|c|c|c|c|c|c|}
\hline $\begin{array}{c}\text { Peak } \\
\#\end{array}$ & $\begin{array}{c}\text { RetTime } \\
\text { [min] }\end{array}$ & Type & $\begin{array}{l}\text { Width } \\
\text { [min] }\end{array}$ & $\begin{array}{c}\text { Area } \\
{\left[\mathrm{mAU}^{\star} \mathrm{s}\right]}\end{array}$ & $\begin{array}{l}\text { Height } \\
{[\mathrm{mAU}]}\end{array}$ & $\begin{array}{c}\text { Area } \\
\%\end{array}$ \\
\hline 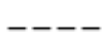 & & & & --1 & -- & ------1 \\
\hline 1 & 12. & MM & 0 & $2.05640 \mathrm{e} 4$ & 813.1 & 50.4391 \\
\hline 2 & 38.270 & $\mathrm{BB}$ & . & $2.02059 e 4$ & 216.76103 & 49.5609 \\
\hline
\end{tabular}

Totals :

$4.07699 e 4 \quad 1029.87126$
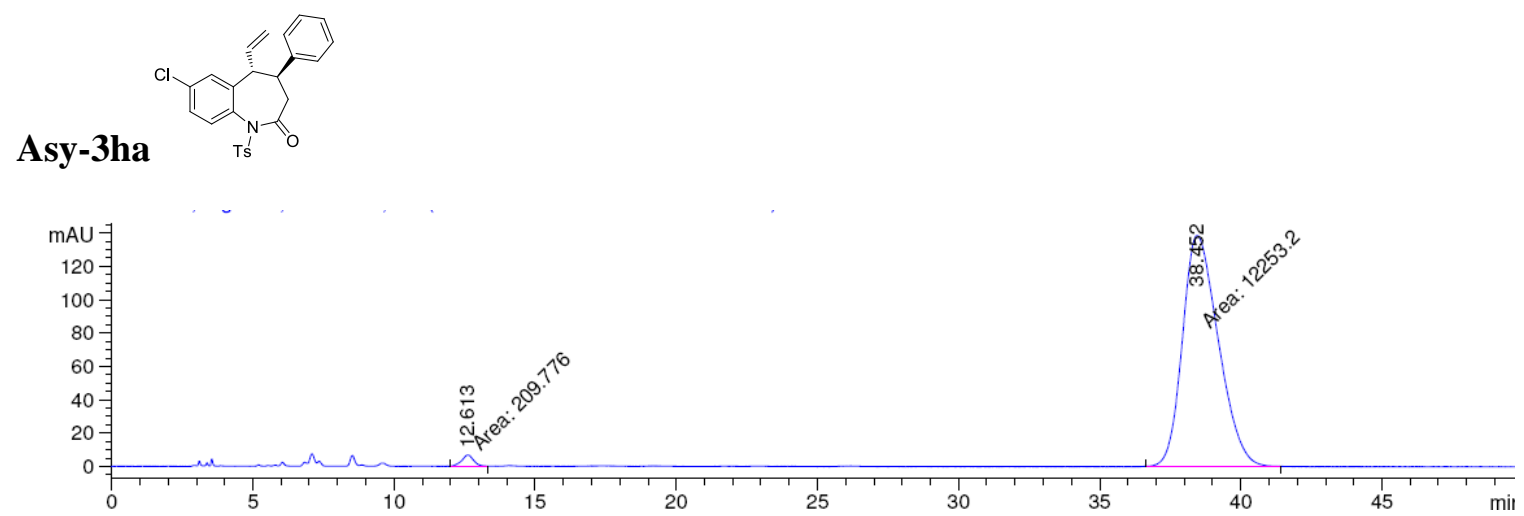

\begin{tabular}{|c|c|c|c|c|c|c|}
\hline $\begin{array}{c}\text { Peak } \\
\#\end{array}$ & $\begin{array}{c}\text { RetTime } \\
\text { [min] }\end{array}$ & Type & $\begin{array}{l}\text { Width } \\
\text { [min] }\end{array}$ & $\begin{array}{c}\text { Area } \\
{\left[\mathrm{mAU}^{*} \mathrm{~s}\right]}\end{array}$ & $\begin{array}{l}\text { Height } \\
{[\mathrm{mAU}]}\end{array}$ & $\begin{array}{c}\text { Area } \\
\%\end{array}$ \\
\hline & & & & & & \\
\hline 7 & 12 & 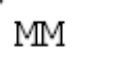 & & 209.77621 & 327 & 32 \\
\hline 2 & 38.452 & MM & 2 & $1.22532 e 4$ & 138.71574 & 98 \\
\hline
\end{tabular}

Totals : $\quad 1.24630 e 4 \quad 145.68902$ 

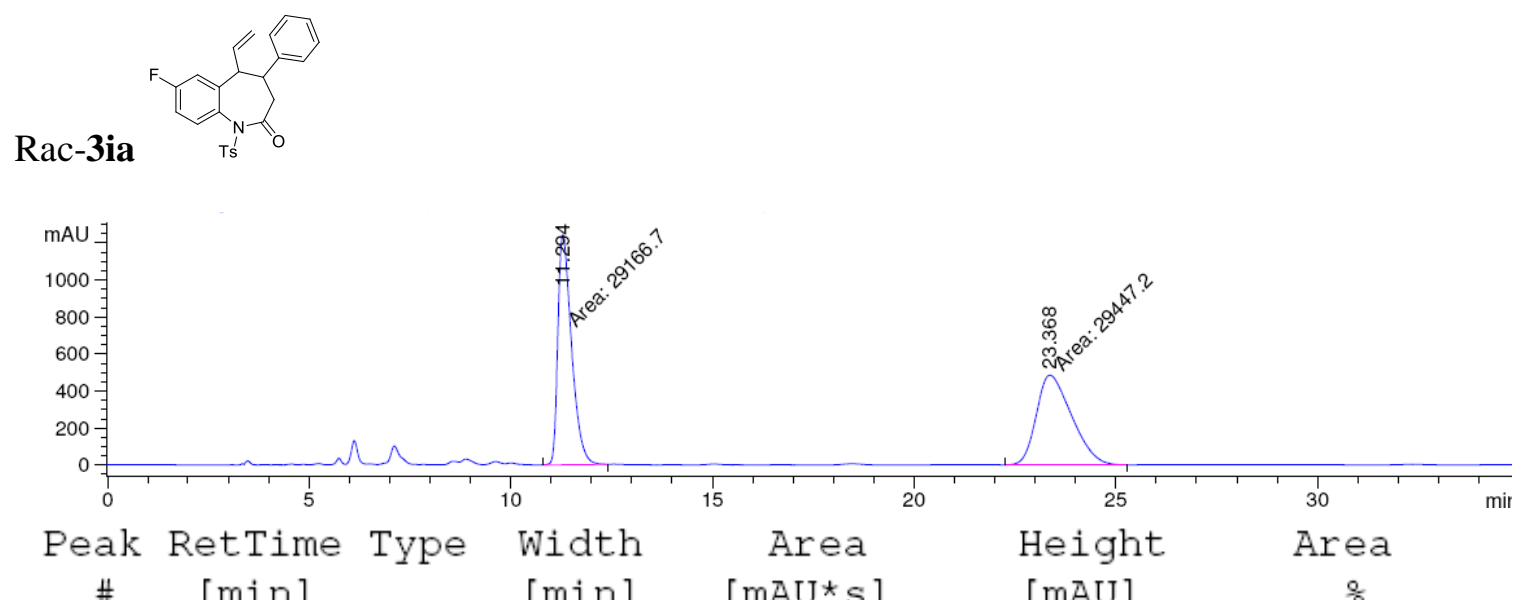

$\begin{array}{ccccccc}\# & {[\mathrm{~min}]} & {[\mathrm{min}]} & {\left[\mathrm{mAU}{ }^{*} \mathrm{~s}\right]} & {[\mathrm{mAU}]} & \% \\ ----\mid & ------|----| & ------\mid & --------|--------| & -------\mid \\ 1 & 11.294 & \mathrm{MM} & 0.3914 & 2.91667 e 4 & 1242.03955 & 49.7607 \\ 2 & 23.368 & \mathrm{MM} & 1.0124 & 2.94472 e 4 & 484.77957 & 50.2393\end{array}$

Totals : $\quad 5.86138 e 4 \quad 1726.81912$
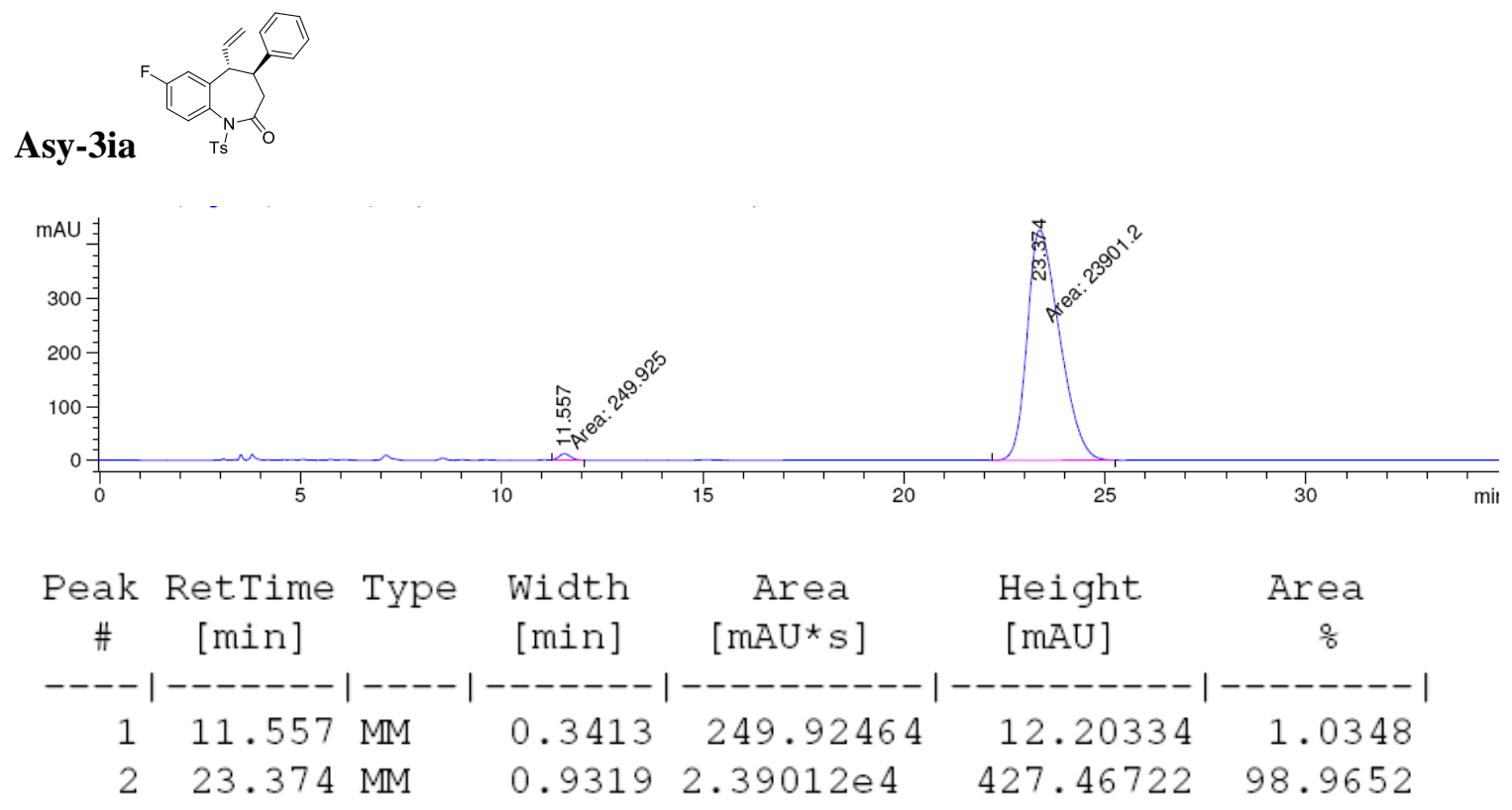

Totals : $\quad 2.41511 e 4 \quad 439.67056$ 

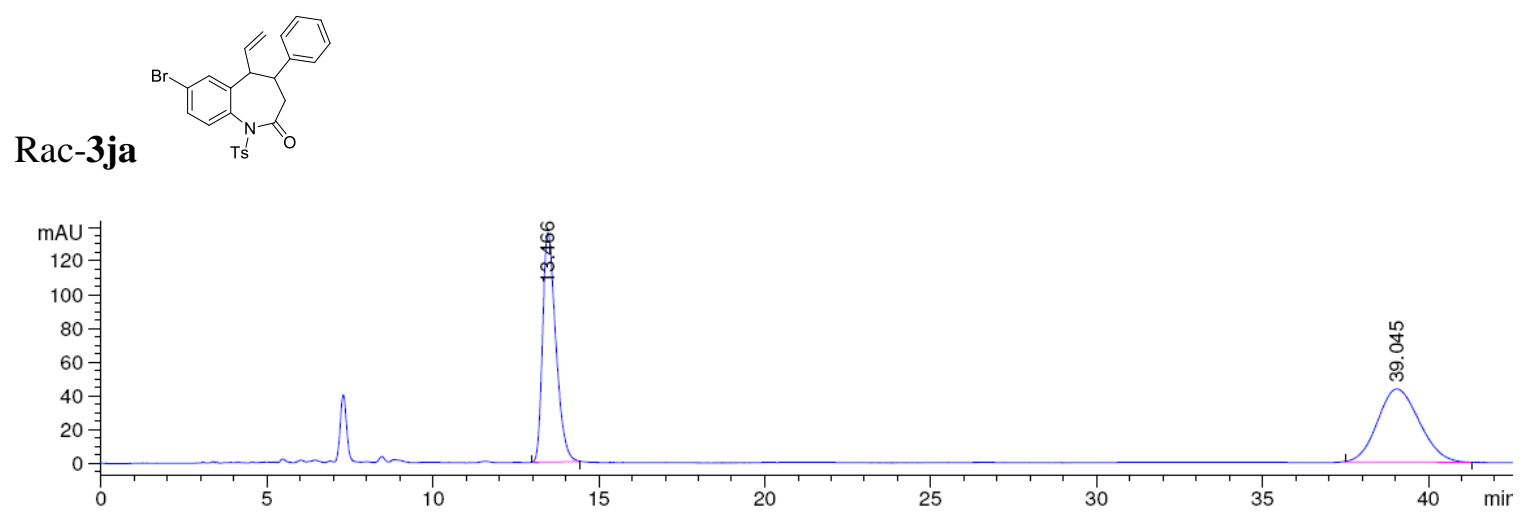

\begin{tabular}{|c|c|c|c|c|c|c|}
\hline $\begin{array}{c}\text { Peak } \\
\#\end{array}$ & $\begin{array}{c}\text { RetTime } \\
\text { [min] }\end{array}$ & Type & $\begin{array}{l}\text { Width } \\
\text { [min] }\end{array}$ & $\begin{array}{c}\text { Area } \\
{\left[\mathrm{mAU}^{*} \mathrm{~s}\right]}\end{array}$ & $\begin{array}{l}\text { Height } \\
\text { [mAU] }\end{array}$ & $\begin{array}{c}\text { Area } \\
\frac{\circ}{\delta}\end{array}$ \\
\hline & & & & & - & . \\
\hline 1 & & B & & 3766 . & 136.0 & 48 \\
\hline 2 & 39.045 & $\mathrm{BB}$ & 1.0230 & 3792.52832 & 43.37919 & 1752 \\
\hline
\end{tabular}

Totals :

$7558.57471 \quad 179.43315$
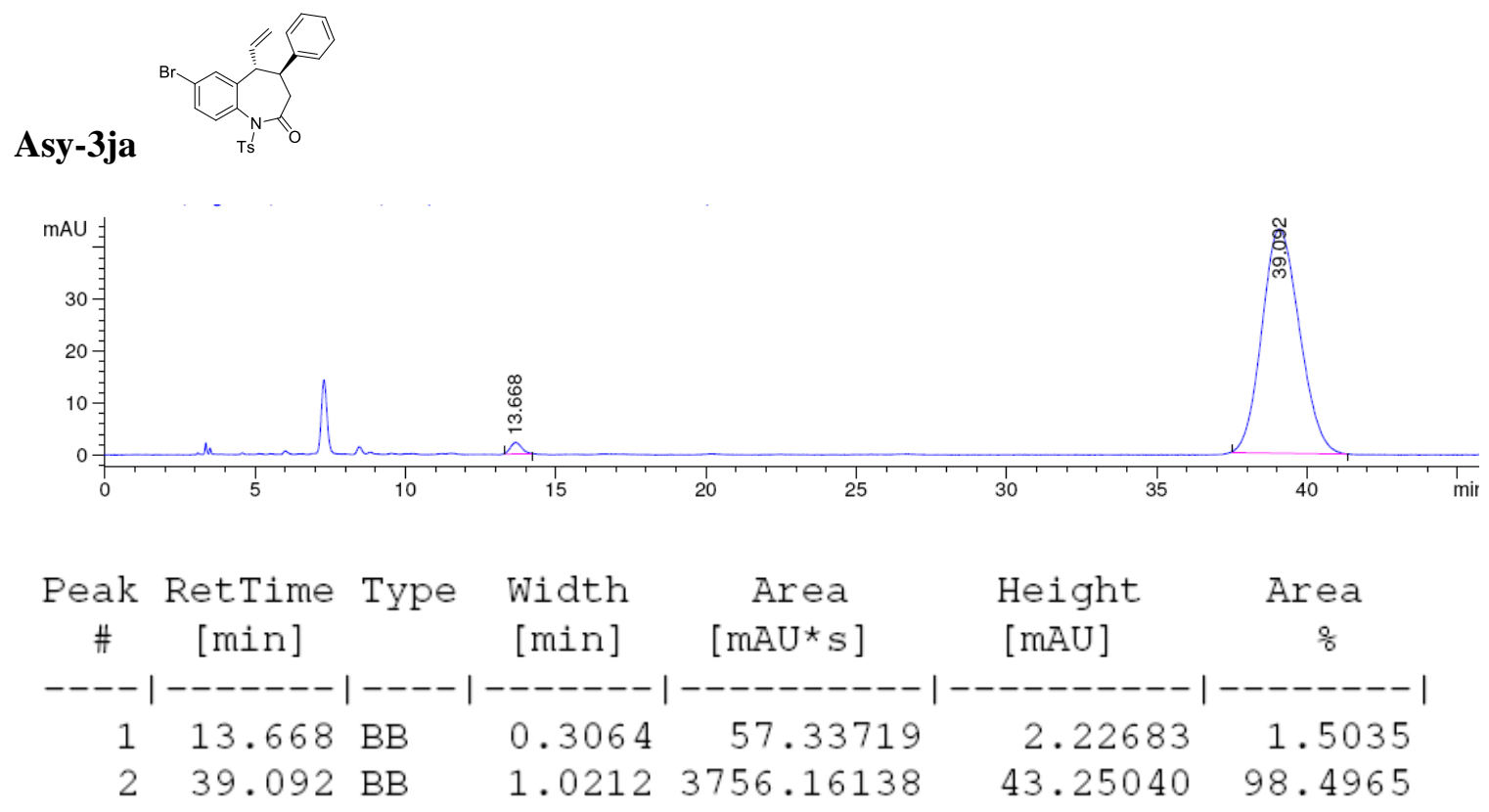

Totals : $\quad 3813.49857 \quad 45.47723$ 

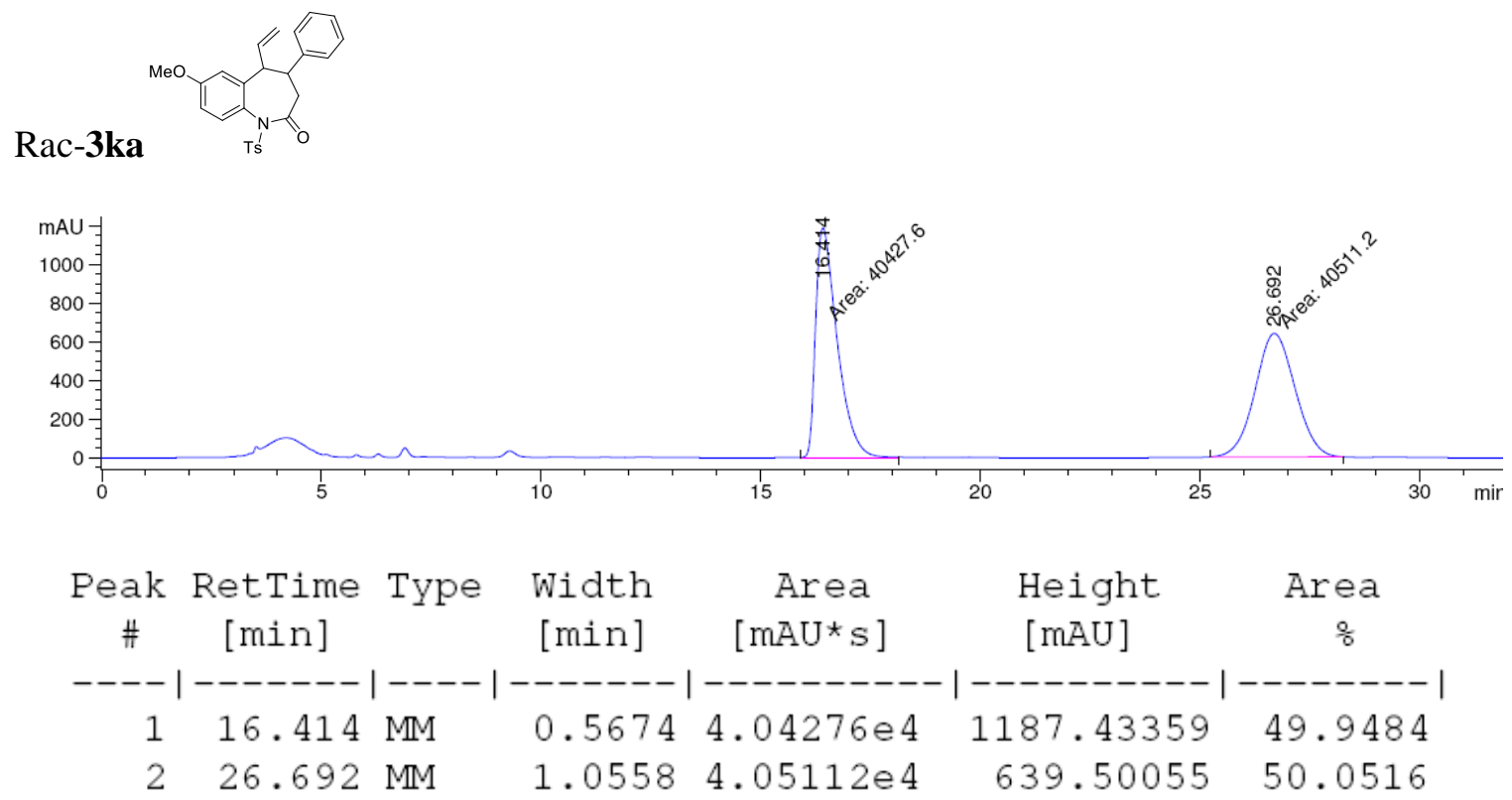

Totals : $\quad 8.09388 e 4 \quad 1826.93414$
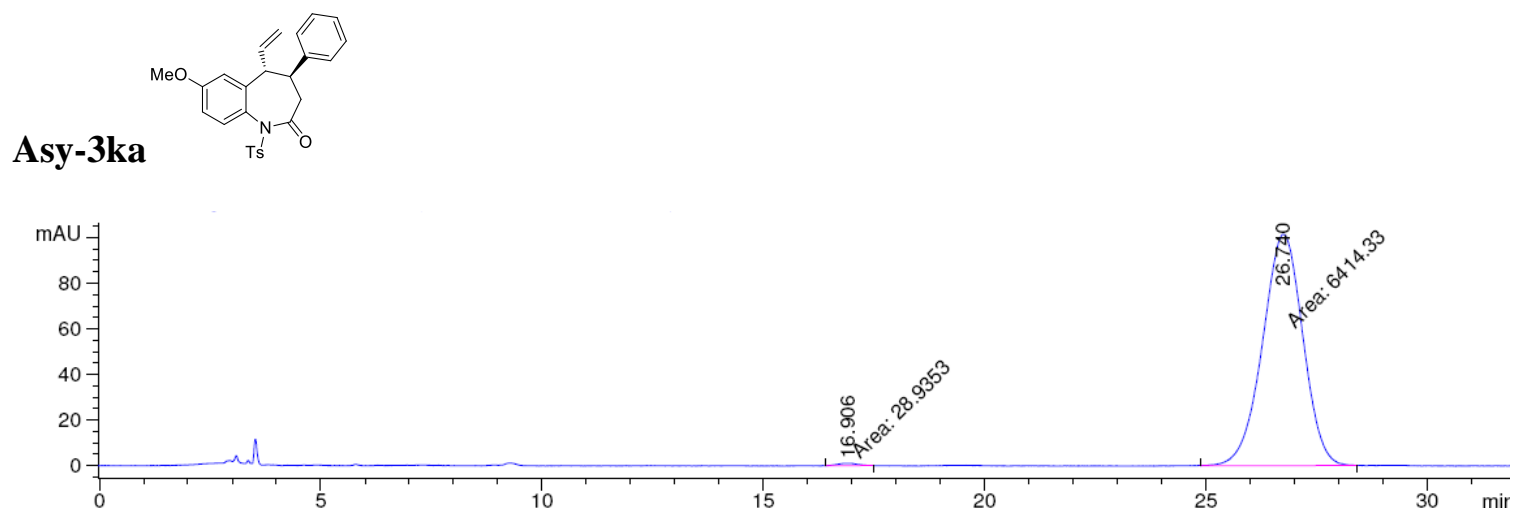

\begin{tabular}{|c|c|c|c|c|c|c|}
\hline $\begin{array}{c}\text { Peak } \\
\#\end{array}$ & $\begin{array}{c}\text { RetTime } \\
\text { [min] }\end{array}$ & Type & $\begin{array}{l}\text { Width } \\
\text { [min] }\end{array}$ & $\begin{array}{c}\text { Area } \\
{\left[\mathrm{mAU}^{*} \mathrm{~s}\right]}\end{array}$ & $\begin{array}{l}\text { Height } \\
{[\mathrm{mAU}]}\end{array}$ & $\begin{array}{c}\text { Area } \\
\%\end{array}$ \\
\hline & & & & & & \\
\hline 1 & & 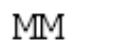 & & & & \\
\hline 2 & 26.740 & $\mathbb{M}$ & 41 & 6414.33105 & 101.41881 & 99.5509 \\
\hline
\end{tabular}

Totals : $\quad 6443.26636 \quad 102.39314$ 

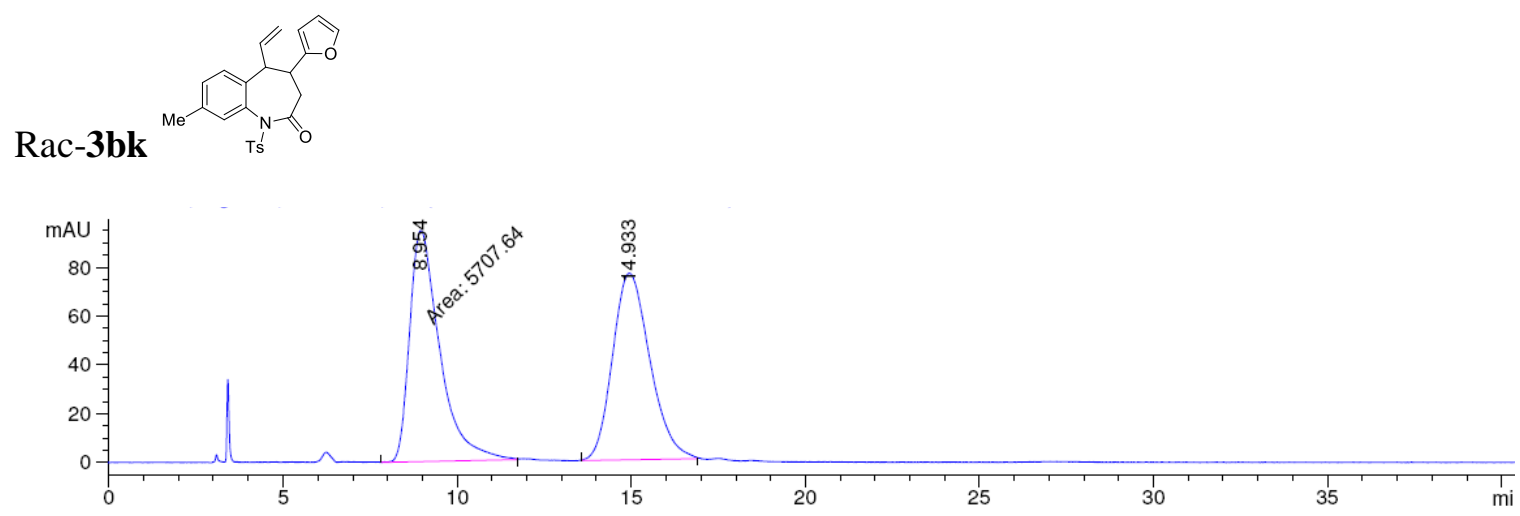

\begin{tabular}{|c|c|c|c|c|c|c|}
\hline $\begin{array}{c}\text { Peak } \\
\#\end{array}$ & $\begin{array}{c}\text { RetTime } \\
\text { [min] }\end{array}$ & Type & $\begin{array}{l}\text { Width } \\
\text { [min] }\end{array}$ & $\begin{array}{c}\text { Area } \\
{\left[\mathrm{mAU}^{\star} \mathrm{s}\right]}\end{array}$ & $\begin{array}{l}\text { Height } \\
{[\mathrm{mAU}]}\end{array}$ & $\begin{array}{c}\text { Area } \\
\frac{\%}{\delta}\end{array}$ \\
\hline & & & & ---- & -- & - \\
\hline 1 & 8.954 & MM & 1 & 5707.63867 & 94.76653 & 50 \\
\hline 2 & 14.933 & $\mathrm{RP}$ & 42 & 5706.60498 & 76.59592 & 9955 \\
\hline
\end{tabular}

Totals : $\quad 1.14142 \mathrm{e} 4 \quad 171.36244$
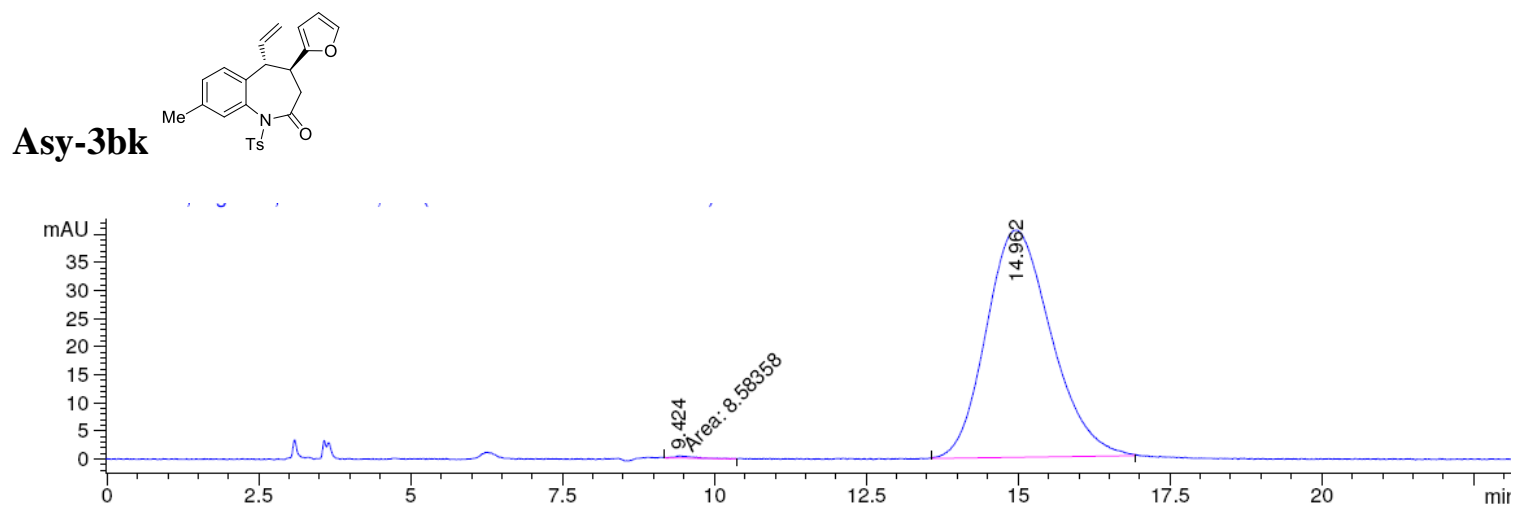

\begin{tabular}{|c|c|c|c|c|c|c|}
\hline $\begin{array}{c}\text { Peak } \\
\#\end{array}$ & $\begin{array}{c}\text { RetTime } \\
\text { [min] }\end{array}$ & Type & $\begin{array}{l}\text { Width } \\
\text { [min] }\end{array}$ & $\begin{array}{c}\text { Area } \\
{\left[\mathrm{mAU}^{*} \mathrm{~s}\right]}\end{array}$ & $\begin{array}{l}\text { Height } \\
{[\mathrm{mAU}]}\end{array}$ & $\begin{array}{c}\text { Area } \\
\%\end{array}$ \\
\hline & & & & - & --1 & \\
\hline 1 & 12 & $\mathrm{MM}$ & 80 & 8.58358 & $3.99571 e-1$ & 33 \\
\hline 2 & 14 & $\mathrm{VB}$ & 46 & 3021.06323 & 10.48502 & 7167 \\
\hline
\end{tabular}

Totals : $\quad 3029.64681 \quad 40.88459$ 

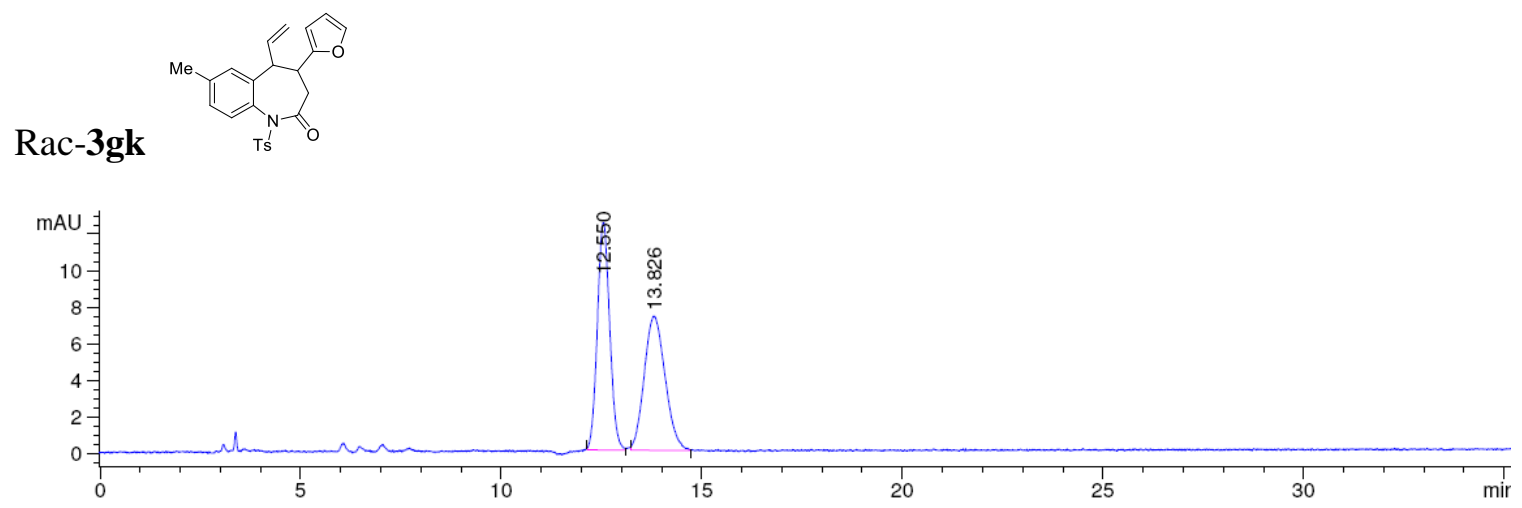

Peak RetTime Type Width Area Height Area

\begin{tabular}{|c|c|c|c|c|c|}
\hline$\#$ & {$[\mathrm{~min}]$} & [min] & {$\left[\mathrm{mAU}^{*} \mathrm{~s}\right]$} & [mAU] & $\%$ \\
\hline & & & & & \\
\hline 1 & $12.550 \mathrm{BB}$ & 0 . & 266.49734 & 2.45244 & 50.1 \\
\hline 2 & $13.826 \mathrm{BB}$ & 0.4264 & 265.29187 & 7.34447 & 49.8867 \\
\hline
\end{tabular}

Totals : $\quad 531.78922 \quad 19.79690$
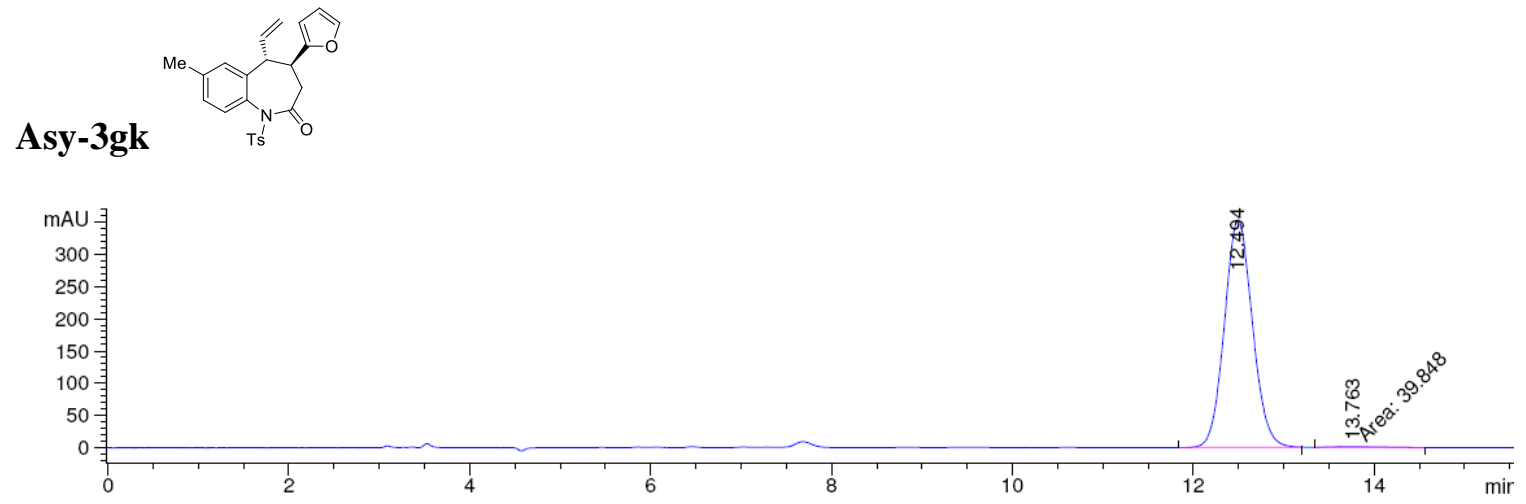

\begin{tabular}{|c|c|c|c|c|c|c|}
\hline $\begin{array}{c}\text { Peak } \\
\#\end{array}$ & $\begin{array}{c}\text { RetTime } \\
\quad[\text { min] }\end{array}$ & Type & $\begin{array}{c}\text { Width } \\
\text { [min] }\end{array}$ & $\begin{array}{c}\text { Area } \\
{\left[\mathrm{mAU}{ }^{*} \mathrm{~s}\right]}\end{array}$ & $\begin{array}{c}\text { Height } \\
{[\mathrm{mAU}]}\end{array}$ & $\begin{array}{c}\text { Area } \\
\%\end{array}$ \\
\hline & $x^{-1}$ & & & ---------- & ---------- & $--------\mid$ \\
\hline 1 & 12.494 & $\mathrm{BB}$ & 0.3308 & 7582.57959 & 352.69458 & 99.4772 \\
\hline 2 & 13.763 & $\mathrm{MM}$ & 0.5861 & 39.84800 & 1.13308 & 0.5228 \\
\hline
\end{tabular}

Totals :

$7622.42759 \quad 353.82766$ 

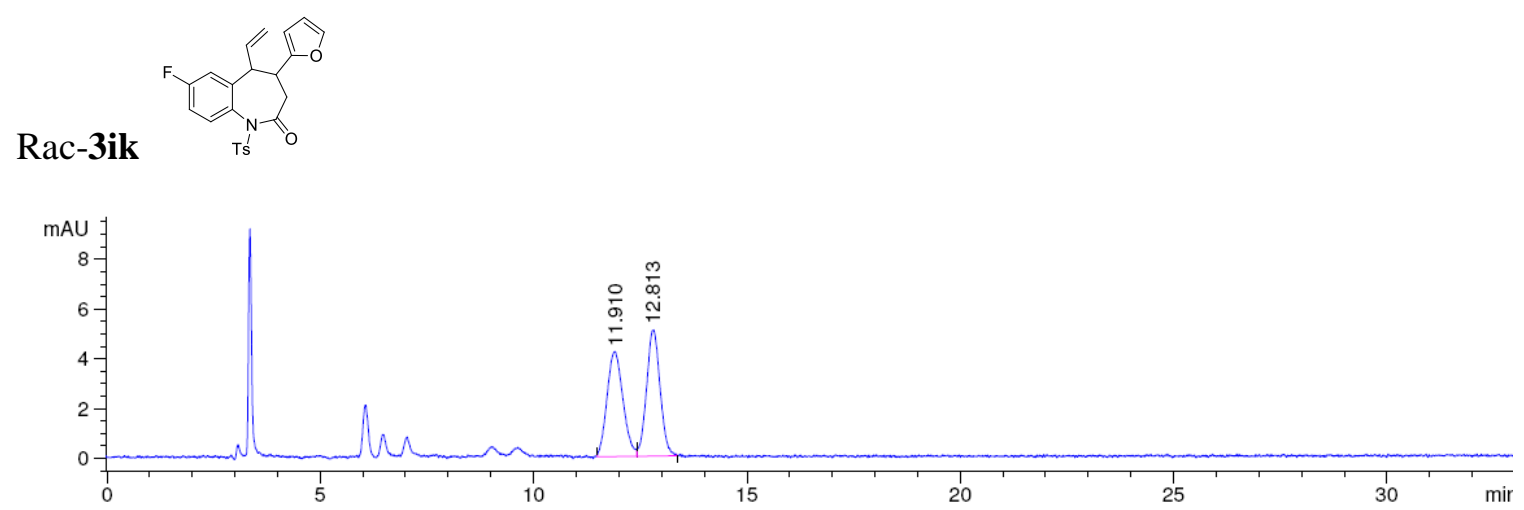

\begin{tabular}{|c|c|c|c|c|c|c|}
\hline $\begin{array}{c}\text { Peak } \\
\#\end{array}$ & $\begin{array}{c}\text { RetTime } \\
\text { [min] }\end{array}$ & Type & $\begin{array}{l}\text { Width } \\
\text { [min] }\end{array}$ & $\begin{array}{c}\text { Area } \\
{\left[\mathrm{mAU}^{\star} \mathrm{s}\right]}\end{array}$ & $\begin{array}{l}\text { Height } \\
{[\mathrm{mAU}]}\end{array}$ & $\begin{array}{c}\text { Area } \\
\%\end{array}$ \\
\hline & & & & --------- & -- & $-------\mid$ \\
\hline 1 & 11.910 & BV & & 109.25404 & 373 & 5211 \\
\hline 2 & 12.813 & VB & & 111.36715 & 5.06949 & 50.4789 \\
\hline
\end{tabular}

Totals : $\quad 220.62119 \quad 9.27822$
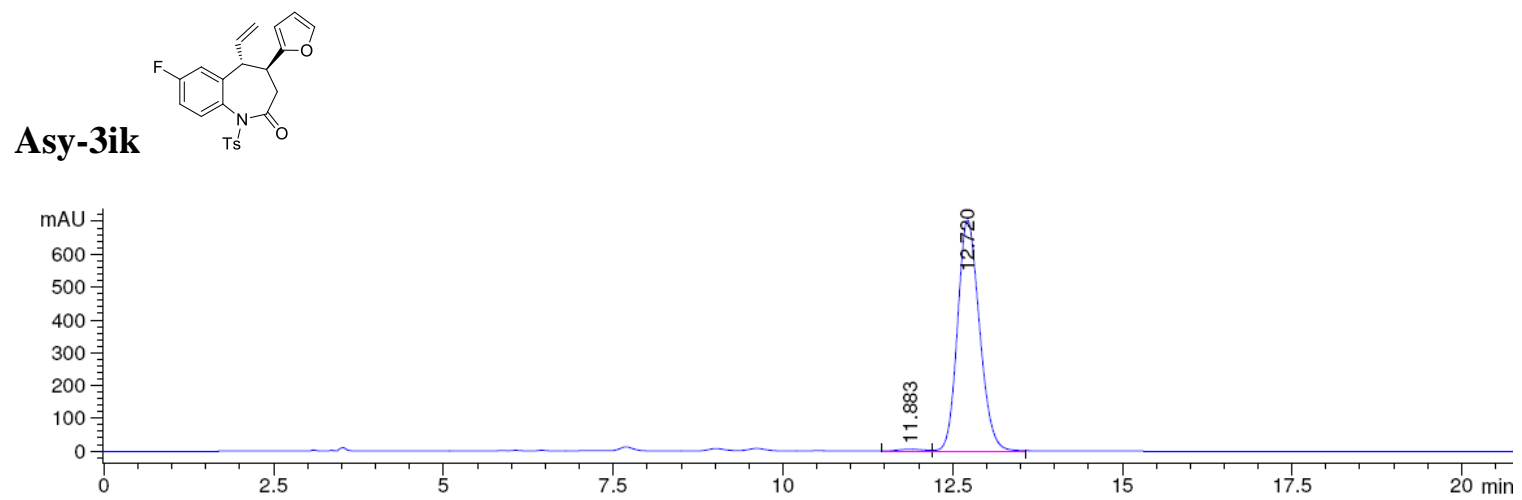

\begin{tabular}{|c|c|c|c|c|c|c|}
\hline $\begin{array}{c}\text { Peak } \\
\#\end{array}$ & $\begin{array}{c}\text { RetTime } \\
\text { [min] }\end{array}$ & Type & $\begin{array}{l}\text { Width } \\
\text { [min] }\end{array}$ & $\begin{array}{c}\text { Area } \\
{\left[\mathrm{mAU}^{*} \mathrm{~s}\right]}\end{array}$ & $\begin{array}{l}\text { Height } \\
{[\mathrm{mAU}]}\end{array}$ & $\begin{array}{c}\text { Area } \\
\%\end{array}$ \\
\hline- & & & & & & \\
\hline 1 & 2 & . & & 3295 & 108 & 224 \\
\hline 2 & 2.720 & & 1 & $1.57597 e 4$ & 704.20758 & .776 \\
\hline
\end{tabular}

Totals :

$1.58904 \mathrm{e} 4 \quad 709.56466$ 

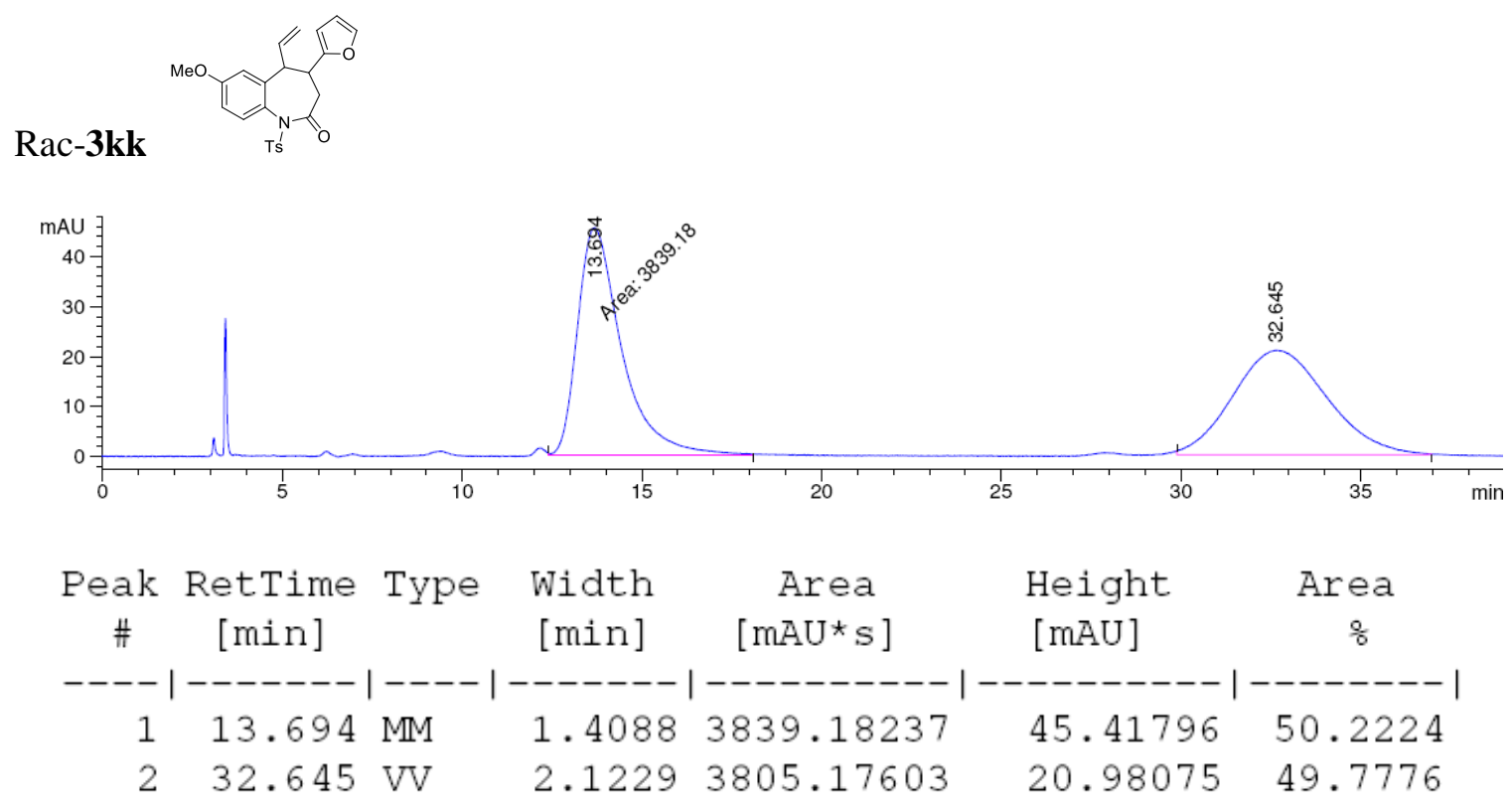

Totals :

$7644.35840 \quad 66.39871$
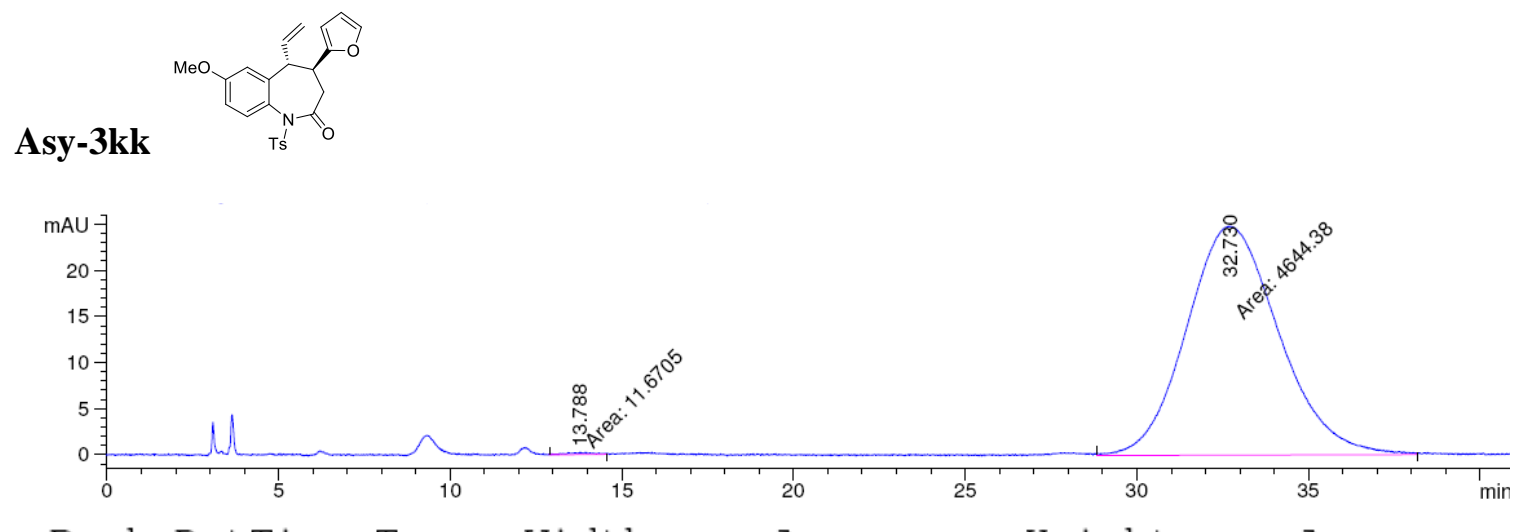

Peak RetTime Type Width Area Height Area

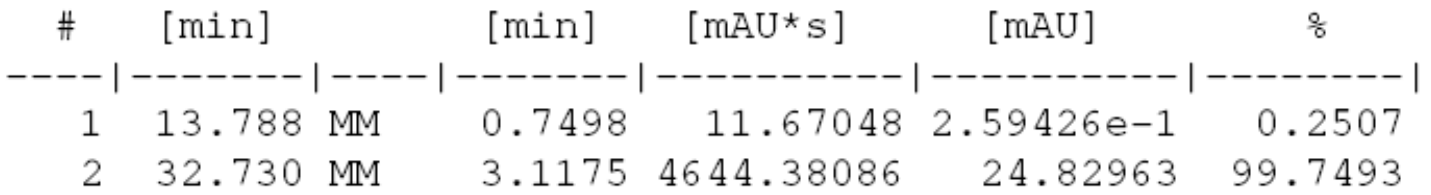

Totals : $\quad 4656.05134 \quad 25.08906$ 

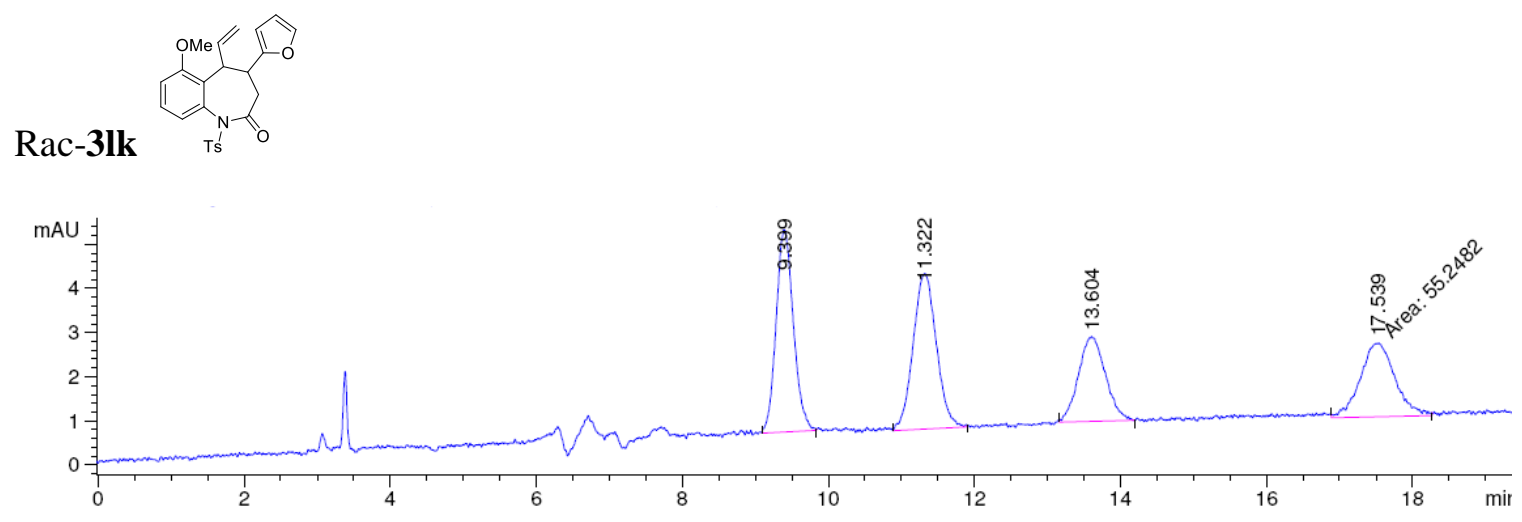

\begin{tabular}{|c|c|c|c|c|c|c|}
\hline $\begin{array}{c}\text { Peak } \\
\#\end{array}$ & $\begin{array}{c}\text { RetTime } \\
\text { [min] }\end{array}$ & Type & $\begin{array}{l}\text { Width } \\
\text { [min] }\end{array}$ & $\begin{array}{c}\text { Area } \\
{\left[\mathrm{mAU}^{\star} \mathrm{s}\right]}\end{array}$ & $\begin{array}{l}\text { Height } \\
{[\mathrm{mAU}]}\end{array}$ & $\begin{array}{c}\text { Area } \\
\%\end{array}$ \\
\hline & ----- & & & & 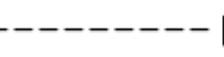 & $--------\mid$ \\
\hline 1 & 9.399 & $\mathrm{BB}$ & 0.2061 & 75.40624 & 4.58066 & 29.2199 \\
\hline 2 & 11.322 & $\mathrm{BB}$ & 0.2652 & 78.10867 & 3.52963 & 30.2671 \\
\hline 3 & 13.604 & $\mathrm{BB}$ & 0.3048 & 49.30169 & 1.92527 & 19.1044 \\
\hline 4 & 17.539 & MM & 0.5505 & 55.24816 & 1.67258 & 21.4086 \\
\hline
\end{tabular}

Totals : $\quad 258.06475 \quad 11.70813$
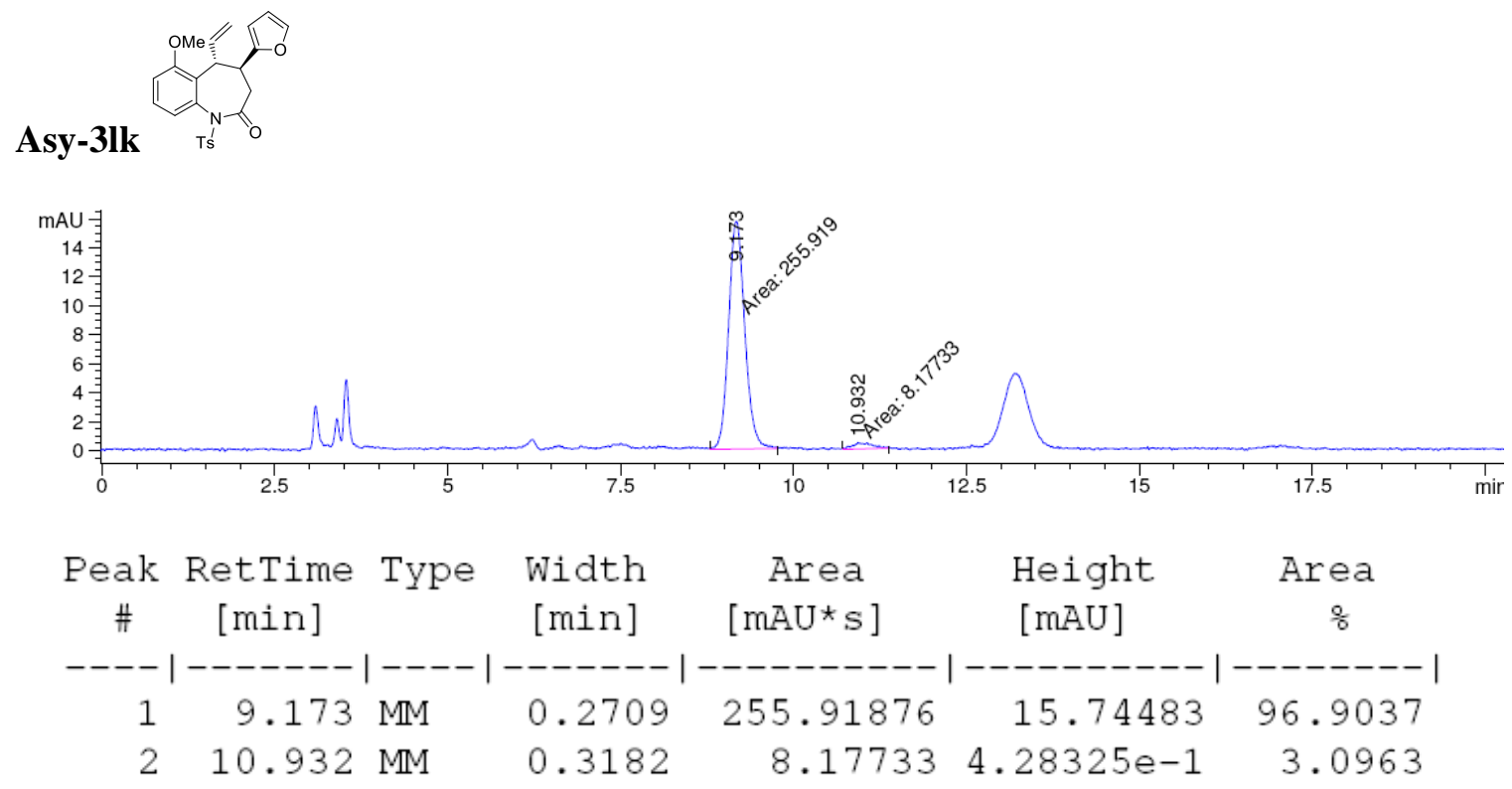

Totals :

$264.09610 \quad 16.17316$ 

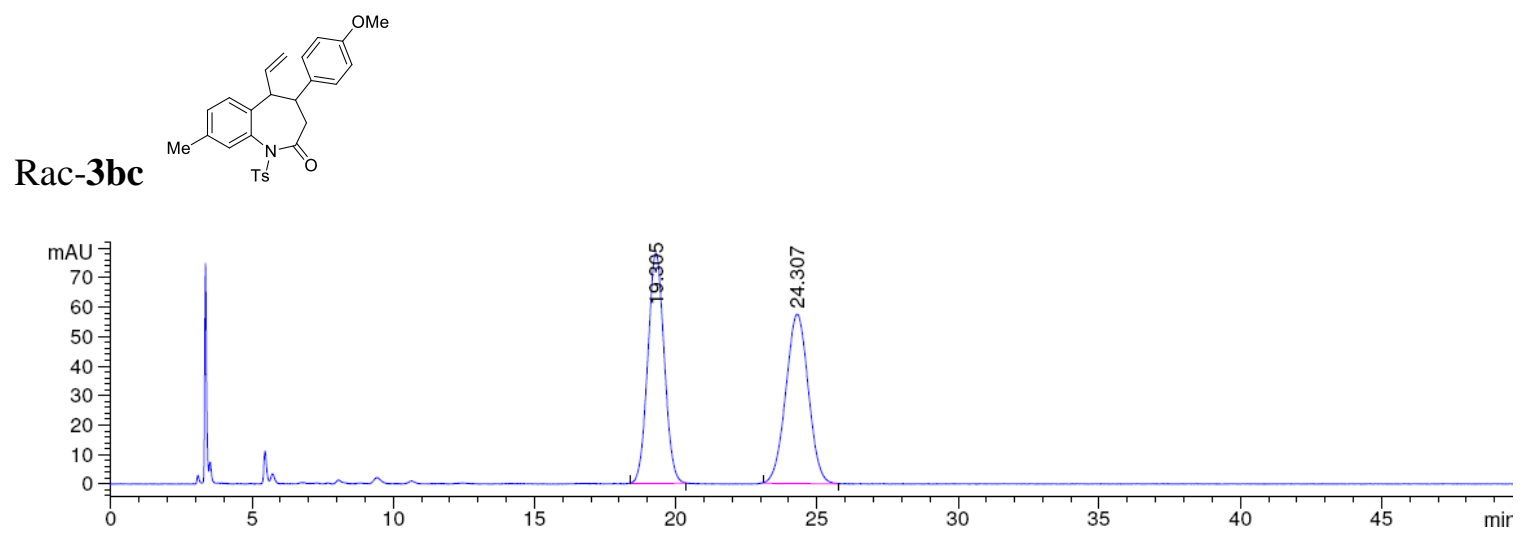

\begin{tabular}{|c|c|c|c|c|c|c|}
\hline $\begin{array}{c}\text { Peak } \\
\#\end{array}$ & $\begin{array}{c}\text { RetTime } \\
\text { [min] }\end{array}$ & Type & $\begin{array}{l}\text { Width } \\
\text { [min] }\end{array}$ & $\begin{array}{c}\text { Area } \\
{\left[\mathrm{mAU}^{*} \mathrm{~s}\right]}\end{array}$ & $\begin{array}{l}\text { Height } \\
\text { [mAU] }\end{array}$ & $\begin{array}{c}\text { Area } \\
\frac{\circ}{6}\end{array}$ \\
\hline & & & & --------2 & 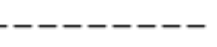 & ---1 \\
\hline 1 & 19.305 & $\mathrm{BB}$ & 0.5894 & 3159.93921 & 78.21268 & 49.8097 \\
\hline 2 & 24.307 & $\mathrm{BB}$ & 0.7116 & 3184.08154 & 57.45226 & 50.1903 \\
\hline
\end{tabular}

Totals :

$6344.02075 \quad 135.66494$
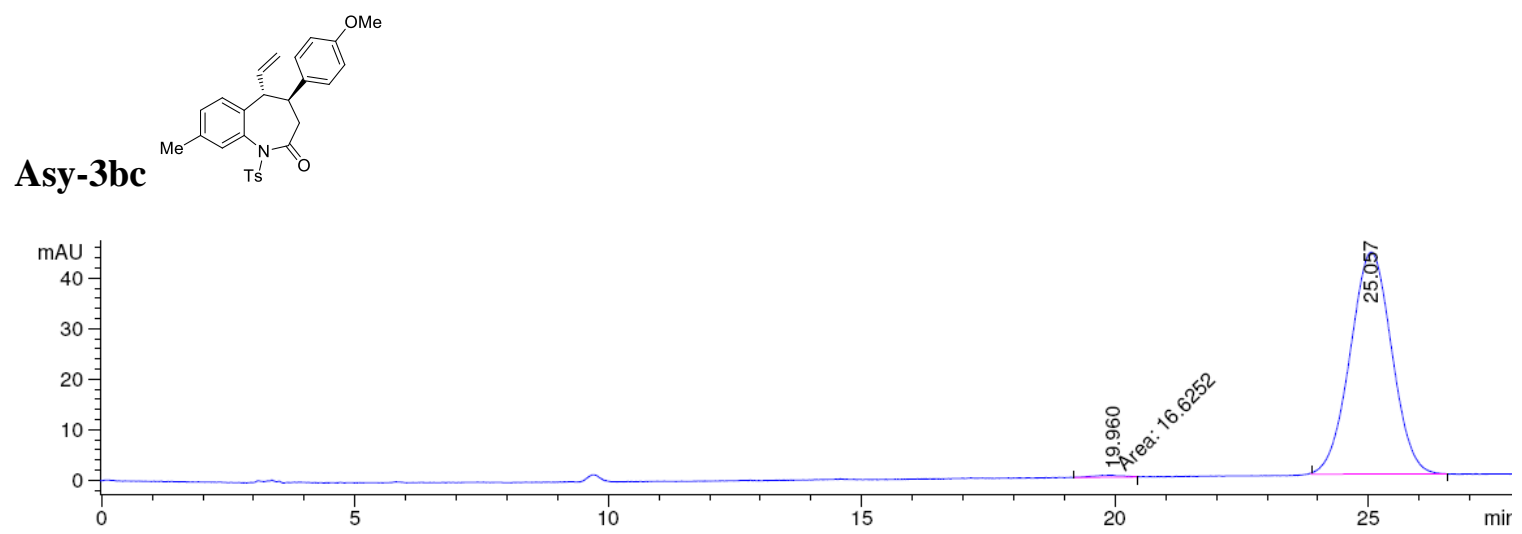

\begin{tabular}{|c|c|c|c|c|c|c|}
\hline $\begin{array}{c}\text { Peak } \\
\#\end{array}$ & $\begin{array}{c}\text { RetTime } \\
\text { [min] }\end{array}$ & Type & $\begin{array}{c}\text { Width } \\
\text { [min] }\end{array}$ & $\begin{array}{c}\text { Area } \\
{\left[\mathrm{mAU}^{*} \mathrm{~s}\right]}\end{array}$ & $\begin{array}{l}\text { Height } \\
{[\mathrm{mAU}]}\end{array}$ & $\begin{array}{c}\text { Area } \\
\%\end{array}$ \\
\hline & & & & --------- & $\mid---$ & -- \\
\hline 1 & 1 & $\mathrm{MM}$ & . & 16.62520 & $e-1$ & 598 \\
\hline 0 & 25.057 & $\mathrm{BF}$ & 75 & 2503.13916 & 4934 & 402 \\
\hline
\end{tabular}

Totals : $\quad 2519.76436 \quad 44.37212$ 

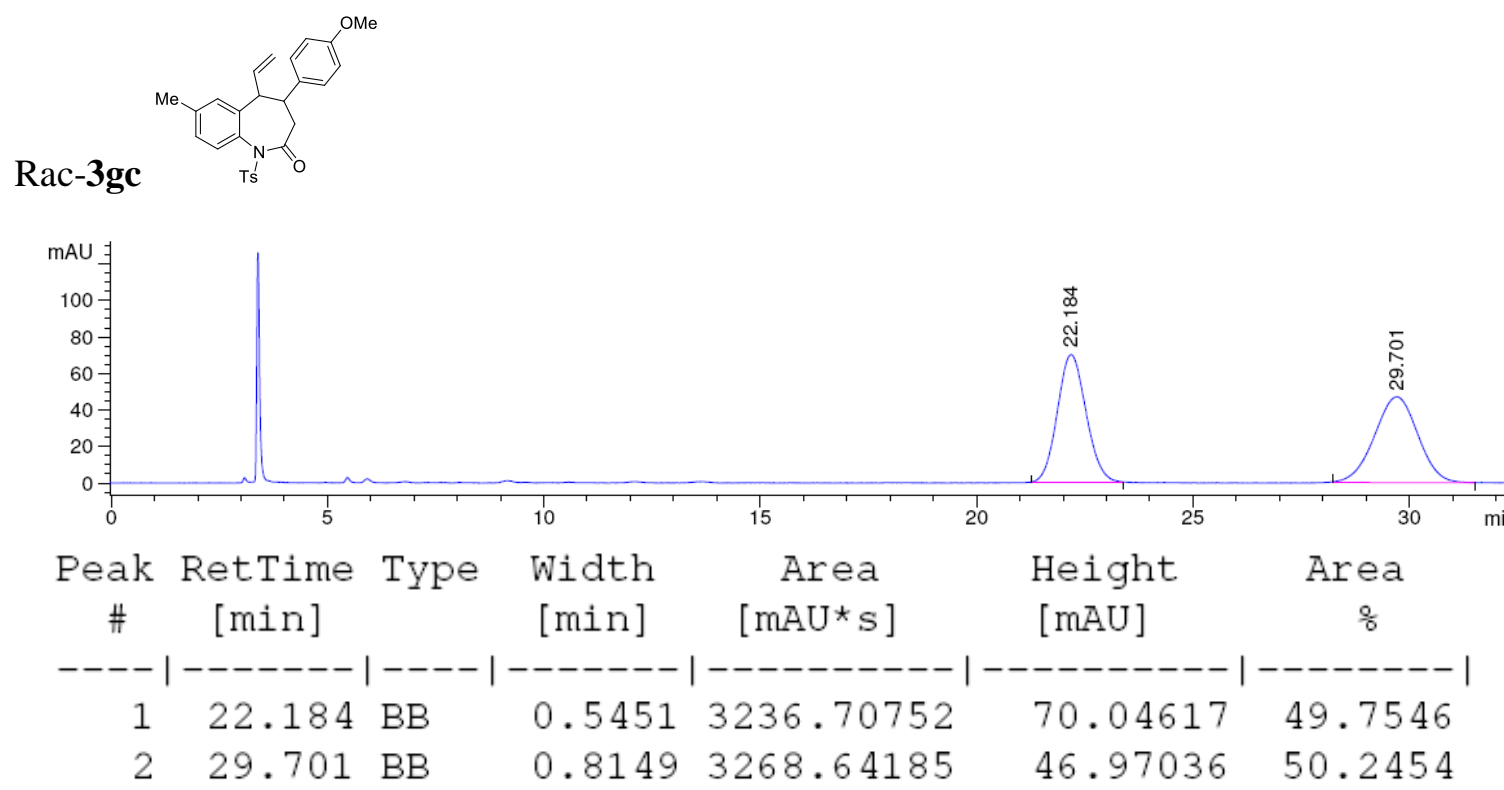

Totals :

$6505.34937 \quad 117.01652$
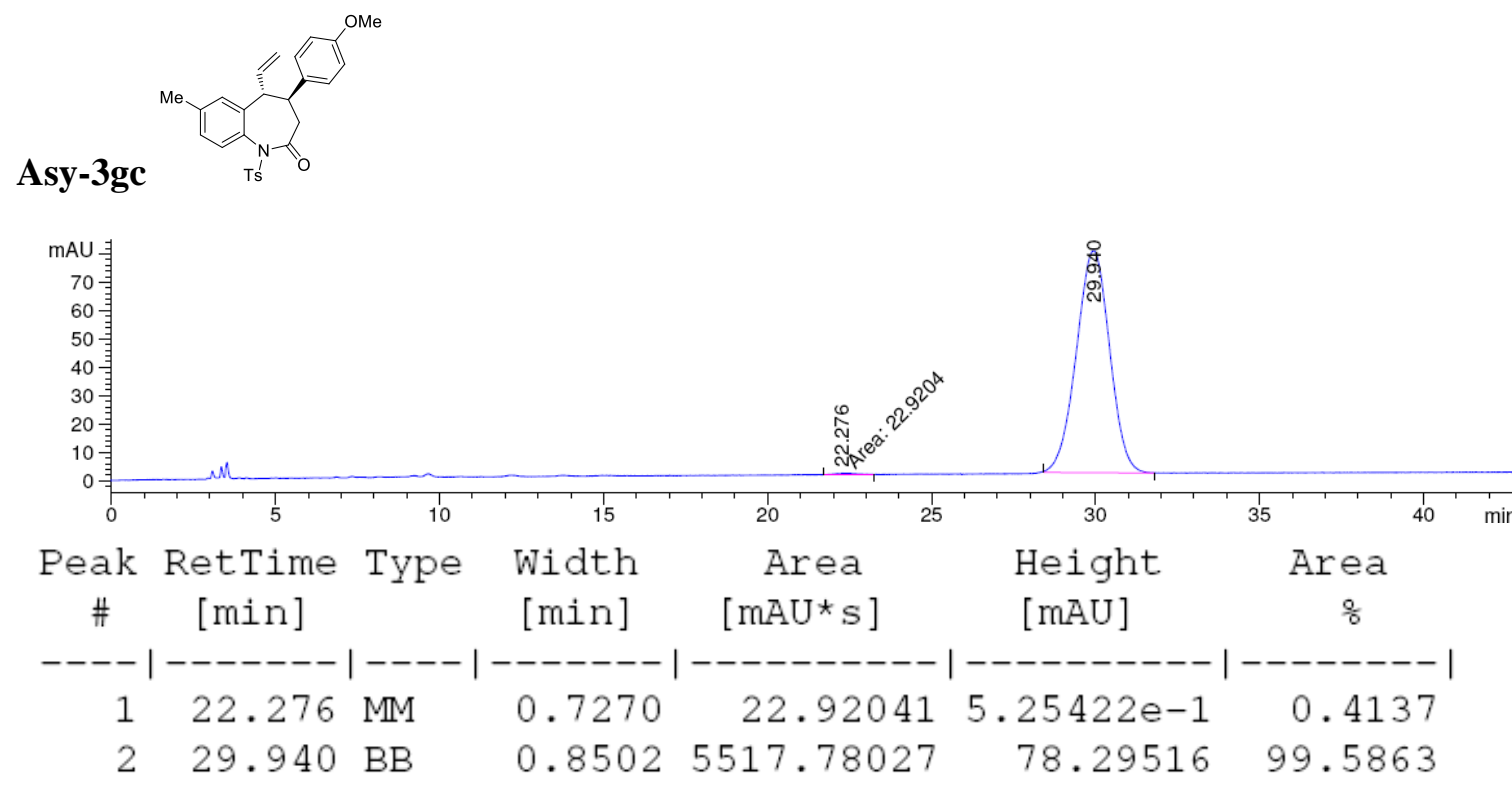

Totals :

$5540.70068 \quad 78.82058$ 

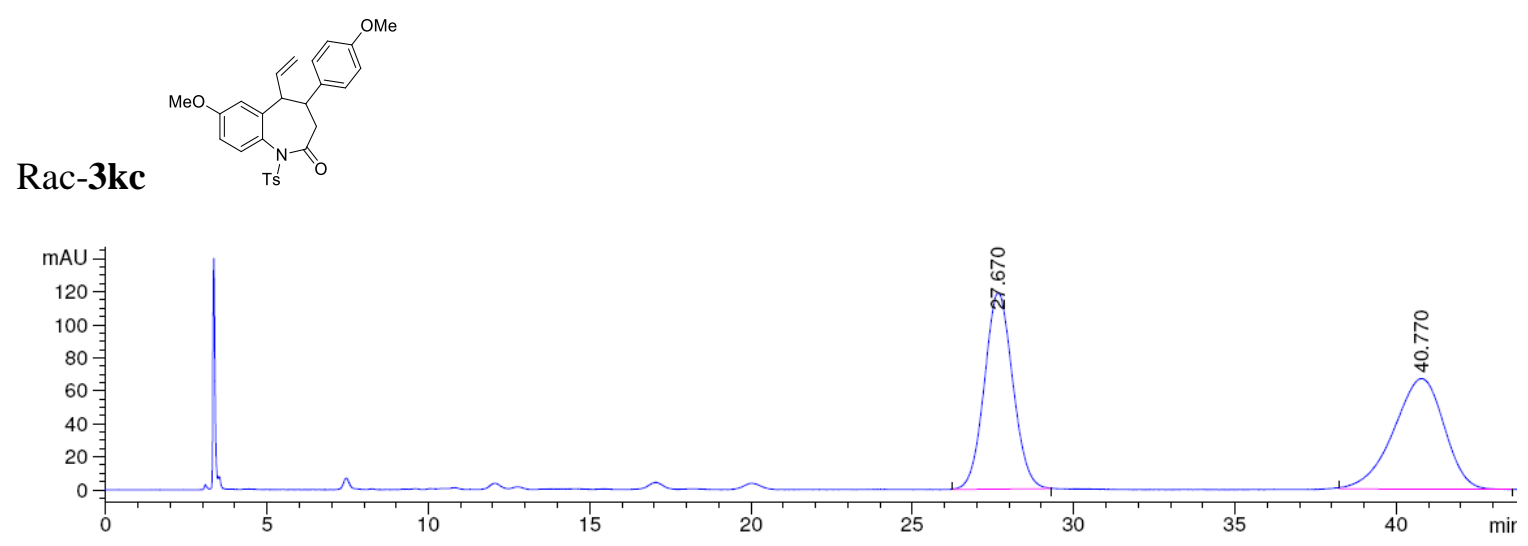

\begin{tabular}{|c|c|c|c|c|c|c|}
\hline $\begin{array}{c}\text { Peak } \\
\#\end{array}$ & $\begin{array}{c}\text { RetTime } \\
\text { [min] }\end{array}$ & Type & $\begin{array}{l}\text { Width } \\
\text { [min] }\end{array}$ & $\begin{array}{c}\text { Area } \\
{\left[\mathrm{mAU}^{\star} \mathrm{s}\right]}\end{array}$ & $\begin{array}{l}\text { Height } \\
{[\mathrm{mAU}]}\end{array}$ & $\begin{array}{c}\text { Area } \\
\%\end{array}$ \\
\hline & & & & & & \\
\hline 1 & & & & 7265. & 118 & \\
\hline 2 & 10.770 & DD & 01 & 7320.11768 & 66.97527 & 1856 \\
\hline
\end{tabular}

Totals : $\quad 1.45861 \mathrm{e} 4 \quad 185.84692$
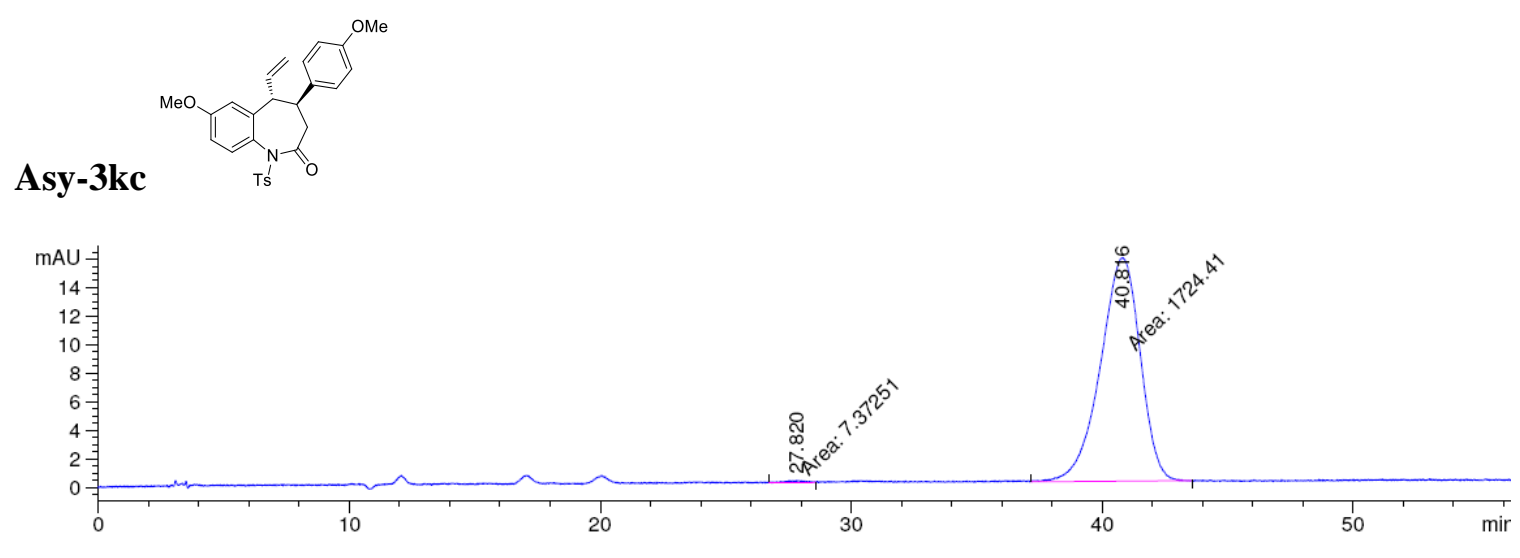

\begin{tabular}{|c|c|c|c|c|c|c|}
\hline $\begin{array}{c}\text { Peak } \\
\#\end{array}$ & $\begin{array}{c}\text { RetTime } \\
\text { [min] }\end{array}$ & Type & $\begin{array}{l}\text { Width } \\
\text { [min] }\end{array}$ & $\begin{array}{c}\text { Area } \\
{\left[\mathrm{mAU}^{*} \mathrm{~s}\right]}\end{array}$ & $\begin{array}{l}\text { Height } \\
\text { [mAU] }\end{array}$ & $\begin{array}{c}\text { Area } \\
\quad \%\end{array}$ \\
\hline & & & & & & \\
\hline 2 & & $\mathbb{M}$ & & $\begin{array}{r}7.37251 \\
1724.41467\end{array}$ & 15.66833 & 5743 \\
\hline
\end{tabular}

Totals :

$1731.78718 \quad 15.82834$ 

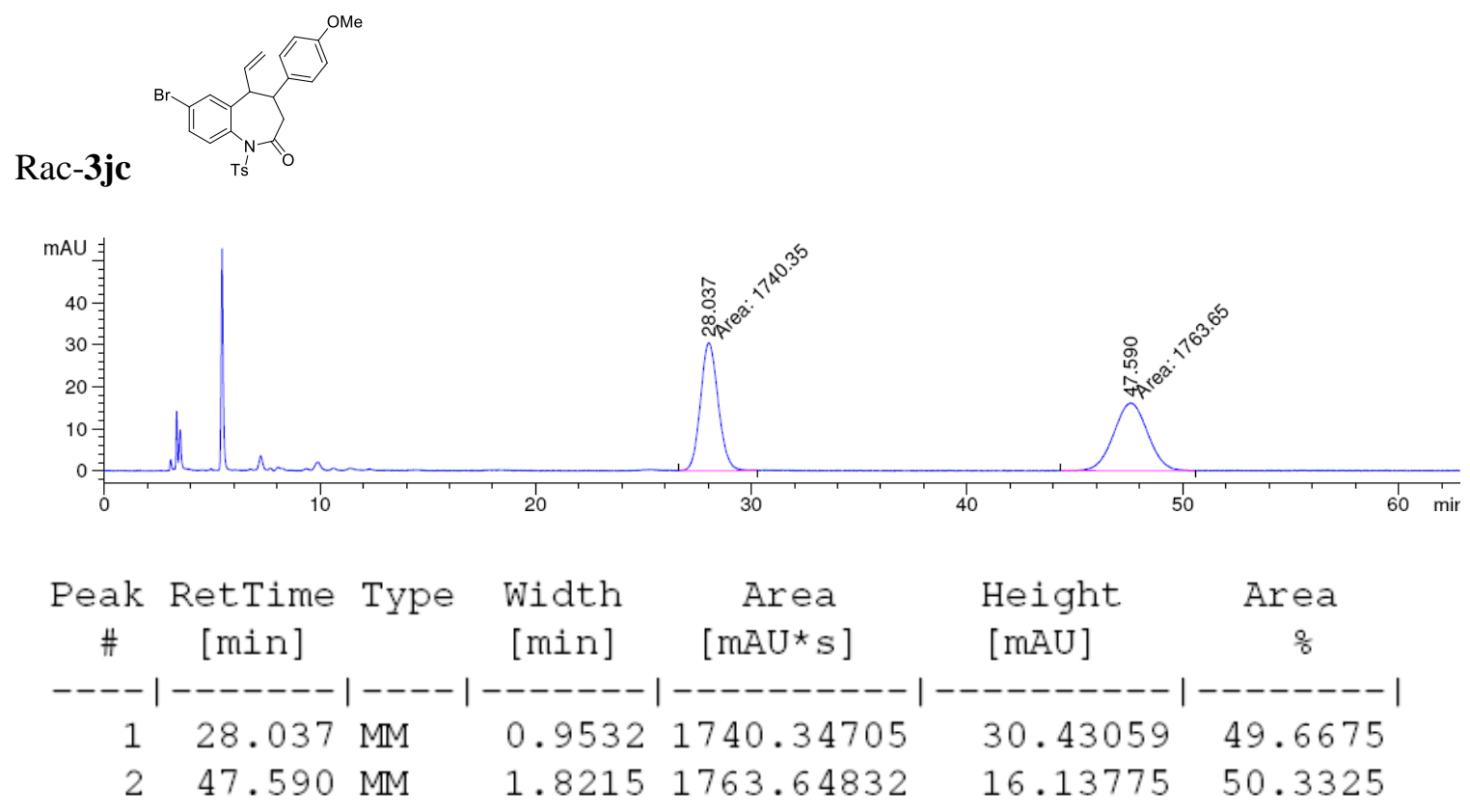

Totals :

$3503.99536 \quad 46.56834$
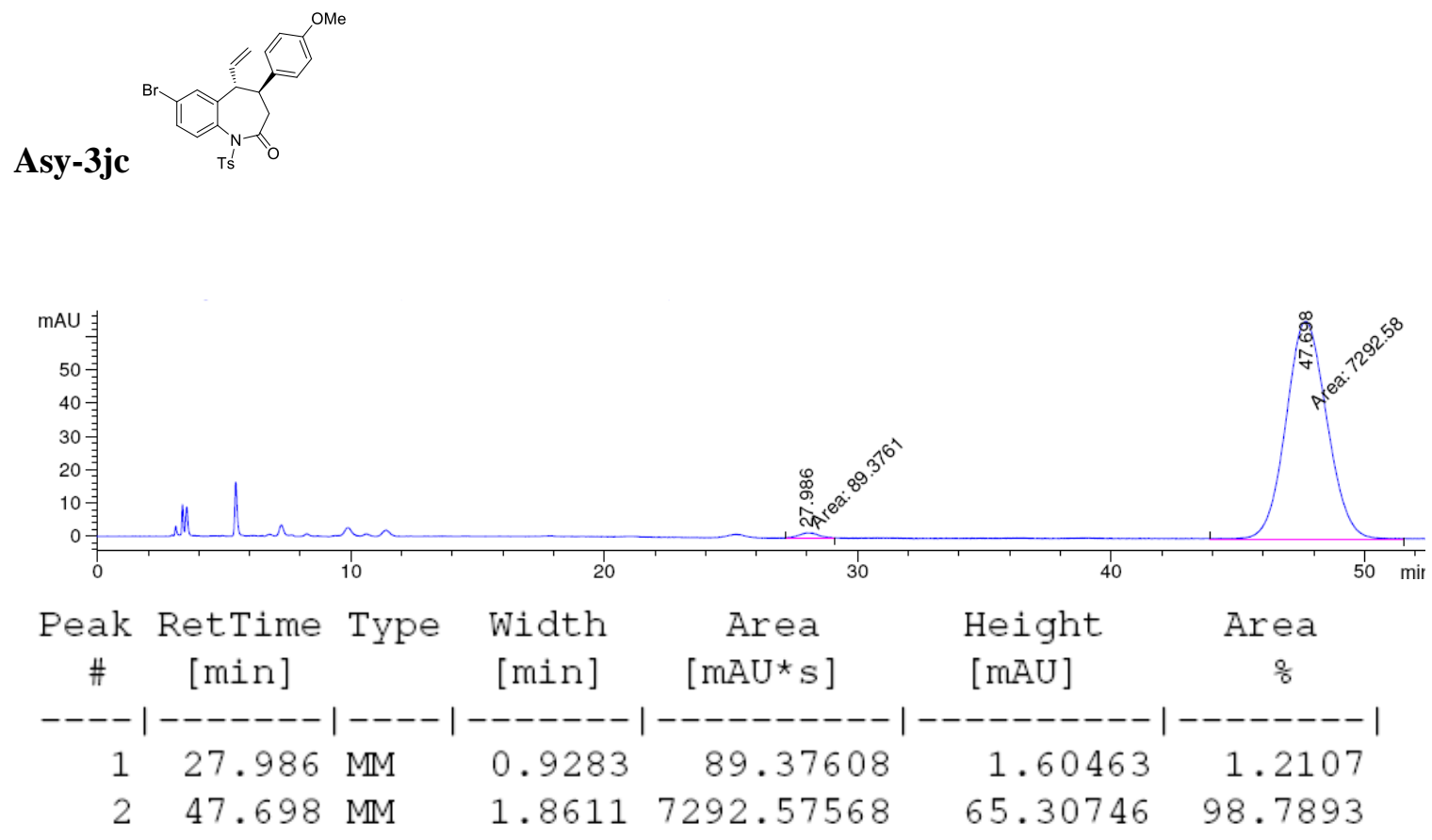

Totals :

$7381.95177 \quad 66.91208$ 

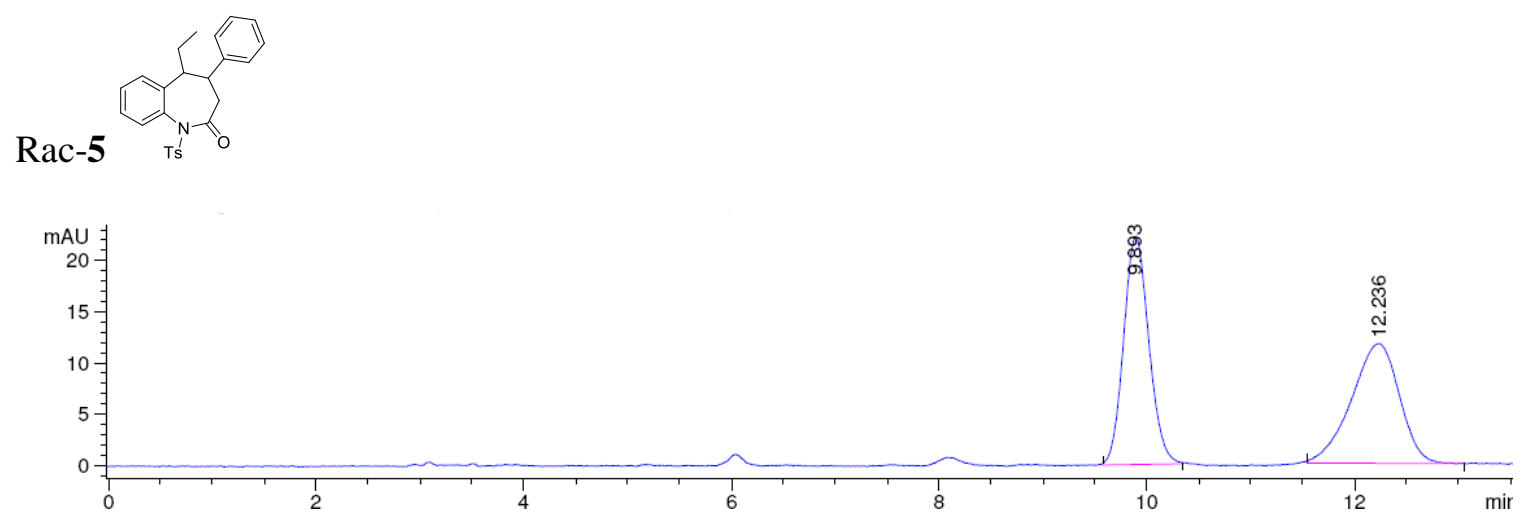

\begin{tabular}{|c|c|c|c|c|c|c|}
\hline $\begin{array}{c}\text { Peak } \\
\#\end{array}$ & $\begin{array}{c}\text { RetTime } \\
\text { [min] }\end{array}$ & Type & $\begin{array}{l}\text { Width } \\
\text { [min] }\end{array}$ & $\begin{array}{c}\text { Area } \\
{\left[\mathrm{mAU}^{\star} \mathrm{s}\right]}\end{array}$ & $\begin{array}{l}\text { Height } \\
{[\mathrm{mAU}]}\end{array}$ & $\begin{array}{c}\text { Area } \\
\%\end{array}$ \\
\hline 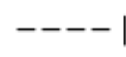 & & & & 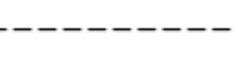 & & 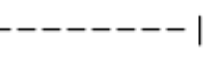 \\
\hline 1 & 9.8 & $3 B$ & & 9036 & 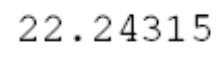 & 50 \\
\hline 2 & 12. & $\mathrm{D}$ & 1 & 374.26334 & 1.70311 & 9782 \\
\hline
\end{tabular}

Totals :

$748.85370 \quad 33.94627$
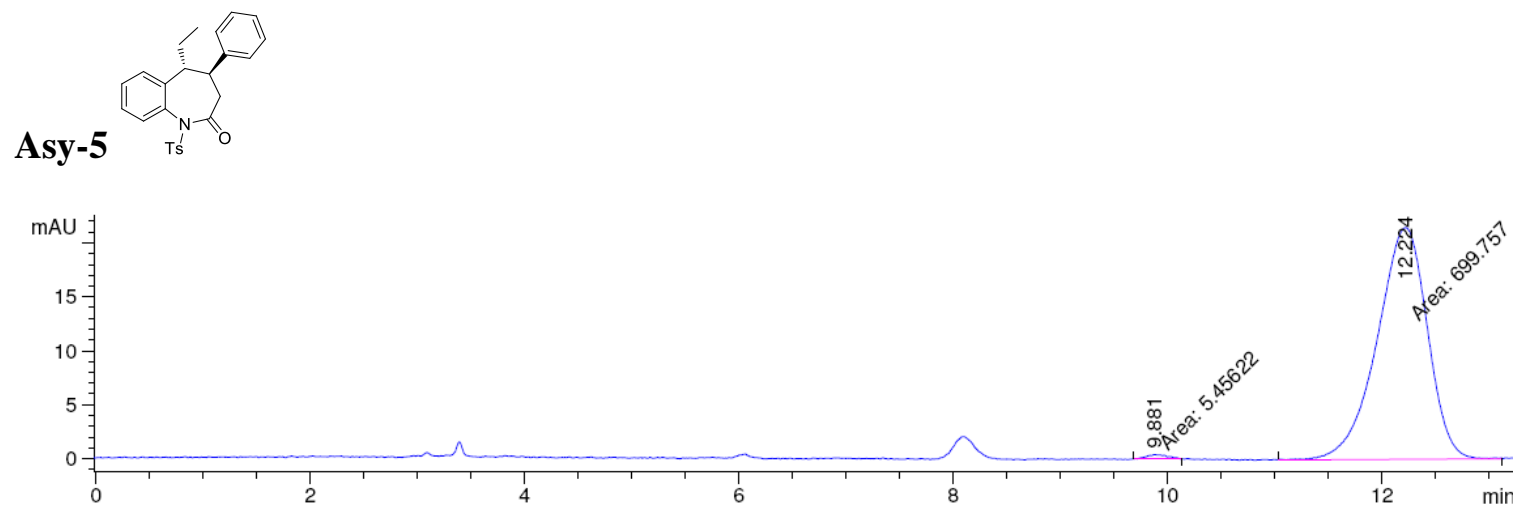

\begin{tabular}{|c|c|c|c|c|c|c|}
\hline $\begin{array}{c}\text { Peak } \\
\#\end{array}$ & $\begin{array}{c}\text { RetTime } \\
\text { [min] }\end{array}$ & Type & $\begin{array}{l}\text { Width } \\
\text { [min] }\end{array}$ & $\begin{array}{c}\text { Area } \\
{\left[\mathrm{mAU}^{\star} \mathrm{s}\right]}\end{array}$ & $\begin{array}{l}\text { Height } \\
{[\mathrm{mAU}]}\end{array}$ & $\begin{array}{c}\text { Area } \\
\%\end{array}$ \\
\hline-- & $-------\mid$ & 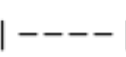 & ------- & ---------- & ---------- & -------- \\
\hline 1 & 9.881 & MM & 0.2316 & 5.45622 & $3.92697 e-1$ & 0.7737 \\
\hline 2 & 12.224 & $\mathrm{MM}$ & 0.5426 & 699.75732 & 21.49530 & 99.2263 \\
\hline Tota. & Ls: & & & 705.21355 & 1.88800 & \\
\hline
\end{tabular}

